\title{
Feasibility study for a recirculating linac-based facility for femtosecond dynamics
}

\author{
December 2002
}

W. Barry, W. A. Barletta, J. Byrd, J. N. Corlett, S. DeSantis, L. Doolittle, W. M. Fawley, M. A. Green, N. Hartman, P. Heimann, D. Kairan ${ }^{1}$, E. Kujawski, S. Leone, D. Li, S. Lidia, P. Luft, R. McClure, H. Padmore, F. Parmigiani ${ }^{2}$, Y. Petroff, W. Pirkl ${ }^{3}$, M. Placidi ${ }^{4}$, D. Reavill, I. Reichel, R. Rimmer, A. Ratti, D. Robin, K. Robinson, F. Sannibale, R. Schoenlein, J. Staples, J. Tanabe, D. Truchlikova, W. Wan, S. Wang, R. Wells, A. Wolski, A. Zholents, M. Zisman.

Lawrence Berkeley National Laboratory, CA, USA.

${ }^{1}$ Budker Institute of Nuclear Physics, Novosibirsk, Russia.

${ }^{2}$ Università Cattolica del Sacro Cuore, Milano, Italy.

${ }^{3}$ Geneva, Switzerland.

${ }^{4}$ CERN, Geneva, Switzerland.

\section{Abstract}

LBNL is pursuing design studies and the scientific program for a facility dedicated to the production of $\mathrm{x}$ ray pulses with ultra-short time duration, for application in dynamical studies of processes in physics, biology, and chemistry. The proposed x-ray facility has the short x-ray pulse length ( $\sim 60$ fs FWHM) necessary to study very fast dynamics, high flux (up to approximately $10^{11}$ photons $/ \mathrm{sec} / 0.1 \% \mathrm{BW}$ ) to study weakly scattering systems, and tuneability over $1-12 \mathrm{keV}$ photon energy. The hard $\mathrm{x}$-ray photon production section of the machine accomodates seven 2-m long undulators. Design studies for longer wavelength sources, using high-gain harmonic generation, are in progress. The x-ray pulse repetition rate of $10 \mathrm{kHz}$ is matched to studies of dynamical processes (initiated by ultra-short laser pulses) that typically have a long recovery time or are not generally cyclic or reversible and need time to allow relaxation, replacement, or flow of the sample. The technique for producing ultra-short x-ray pulses uses relatively long electron bunches to minimise high-peak-current collective effects, and the ultimate x-ray duration is achieved by a combination of bunch manipulation and optical compression. Synchronization of $\mathrm{x}$-ray pulses to sample excitation signals is expected to be of order $50-100 \mathrm{fs}$. Techniques for making use of the recirculating geometry to provide beam-based signals from early passes through the machine are being studied.

\section{Acknowledgment}

This work was supported by the Director, Office of Science, Office of Basic Energy Sciences, of the U.S. Department of Energy under Contract No. DE-AC03-76SF00098.

\section{DISCLAIMER}

This document was prepared as an account of work sponsored by the United States Government. While this document is believed to contain correct information, neither the United States Government nor any agency thereof, nor The Regents of the University of California, nor any of their employees, makes any warranty, express or implied, or assumes any legal responsibility for the accuracy, completeness, or usefulness of any information, apparatus, product, or process disclosed, or represents that its use would not infringe privately owned rights. Reference herein to any specific commercial product, process, or service by its trade name, trademark, manufacturer, or otherwise, does not necessarily constitute or imply its endorsement, recommendation, or favoring by the United States Government or any agency thereof, or The Regents of the University of California. The views and opinions of authors expressed herein do not necessarily state or reflect those of the United States Government or any agency thereof, or The Regents of the University of California. 


\section{Table of Contents}

1. Overview

2. Baseline configuration choices

3. Accelerator physics

4. Collective effects

5. RF photo cathode gun

6. Beam dynamics in the rf gun

7. Experimental studies at FNPL

8. Flat beam adapter

9. Superconducting $\mathrm{rf}$ linacs

10. Deflecting cavities

11. Linac rf systems

12. Photocathode gun rf systems

13. Cryogenics

14. Magnets

15. Vacuum systems

16. Beam diagnostics

17. Lasers

18. Beamlines

19. Synchronization

20. Beam dump

21. Conventional facilities

22. Site selection

23. Technical risk analysis 


\section{List of Figures}

Figure 1-1: Average flux as a function of photon energy. $1-1$

Figure 1-2: Machine layout for the baseline configuration. $1-2$

Figure 1-3: Time/position/angle correlation of the electron bunches. 1-3

Figure 1-4: Compression of the $x$-ray pulse. 1-3

Figure 1-5: X-ray pulse length as a function of photon energy. 1-4

Figure 1-6: UV and soft $x$-ray production technique using high-gain harmonic generation1-9

Figure 2-1: Derivation of systems requirements 2-2

Figure 2-2: Trade-space for baselining the recirculating linac hard $x$-ray configuration2-6

Figure 2-3: Recirculating linac cost trend versus the number of passes 2-8

Figure 3-1: Machine layout, showing injector linac, main linac, four rings, and photon

production section. $3-2$

Figure 3-2: The lattice functions in Ring 1 (first pass through the main linac). 3-3

Figure 3-3: Lattice functions in the main linac for the fourth pass. $3-3$

Figure 3-4: The beam spreader section. 3-5

Figure 3-5: Crossections of a beam spreader and compact quadrupole 3-5

Figure 3-6: Lattice functions for Ring $4 \quad 3-7$

Figure 3-7: Histograms of pathlengths in Arc 3

Figure 3-8: Vertical beam profile at the end of Arc3 for a range of error seeds, no orbit

correction.

Figure 3-9: Vertical beam profile at the end of arc3 for the same range of error seeds, with orbit correction applied.

Figure 3-10: Schematic of the injector system

Figure 3-11: The evolution of the longitudinal phase space

Figure 3-12: Lattice optical functions in the bunch compressor, for $\mathrm{R}_{56}=1.61$.

Figure 3-13: Longitudinal phase space for a compressed electron bunch:

Figure 3-14: The evolution of the longitudinal phase space for a time delay of $3 \mathrm{ps}$ in the input beam

Figure 3-15: The evolution of the longitudinal phase space 3-14

Figure 4-1: Cumulative transverse displacement $4-1$

Figure 4- 2: Displacement at the end of four passes 4-4

Figure 4-3: Electron displacement as a function of the position within the bunch 4-5

Figure 4-4: RMS value of the transverse displacement at the bunch center

Figure 4-5: Electron displacement for zero betatron phase advance

in all arcs

Figure 4-6: Transverse long-range wake averaged over 32 cavities with a frequency spread of $\pm 0.1 \%$.

Figure 4-7: Short and long range transverse wakefields for a 2 ps long bunch.

Figure 4-8: Energy loss of electrons, due to the longitudinal wake, for $2 \mathrm{ps}$ and $20 \mathrm{ps}$ bunches. 
Figure 4-9: Transverse deflections of a 2 ps zero-emittance beam, after four consecutive passes at constant vertical offset through the linac.

Figure 4-10: Results of tracking a nominal bunch, the arc sextupoles were turned on

Figure 4-11: Results of tracking a nominal bunch, the arc sextupoles were turned off

Figure 4-12:. Results of tracking two bunches four passes through the linac and arcs.

Figure 4-13:. Charge distribution and resistive wall wake

Figure 4-14: Transverse deflection for a $1 \mathrm{~m}$ long, $3 \mathrm{~cm}$ diameter vacuum chamber section in the first arc

Figure 4-15:. Deflection along bunches of 1-10 ps length with $1 \mathrm{nC}$ charge

Figure 4-15: Longitudinal density profile of electrons in a bunch.

Figure 4-16: CSR wake function $d E(s) / d z$ for an electron longitudinal density distribution shown in Figure 15.

Figure 5-1: Flat beam injector beamline..

Figure 5-2: Measured peak brightness of recent photoinjectors 5-4

Figure 5-3: 2-D model of RF gun cavities and solenoid magnets 5-5

Figure 5-4: Cell-1 2-D geometry and accelerating mode electric field (SUPERFISH). 5-7

Figure 5-5: 3-D model of RF cell, coupler, and input waveguide 5-9

Figure 5-6: Cavity gradient with 5 microsecond RF pulse, $3.4 \mu$ fill time.

Figure 5-7: ANSYS cavity geometry.

Figure 5-8: ANSYS model of steady-state thermal loading in the RF gun.

Figure 5-9: MAFIA 3-D model of 4-cell RF gun cavities and solenoid coils.

Figure 6-1: Variation of normalized transverse emittance

with bunch charge

Figure 6-2: Variation of normalized transverse emittance with bunch length

Figure 6-3: Variation of normalized transverse emittance with launch phase

Figure 6-4: HOMDYN model of uncompensated (solid) and compensated (dashed) beam dynamics

Figure 6-5: Small angular momentum regime (HOMDYN).

Figure 6-6: The initial thermal emittance and the projected emittance

Figure 7-1: FNPL beamline.

Figure 7-2: Cyclotron phase advance vs. secondary solenoid current 7-3

Figure 7-3: Beam envelope and radial emittance 7-4

Figure 7-4: Vertical emittance vs. cyclotron phase advance 7-5

Figure 7-5: Measured vertical emittances of angular momentum-

Figure 8-1: Drift-cyclotron coordinates $\quad 8-2$

Figure 8-2: Beam spot at slit analyzer and downstream beamlet $\quad 8-7$

Figure 8-3: Beam spot image downstream from the adapter and 
beamlet distribution produced by horizontal slits

Figure 8-4: Beam spot image downstream from the adapter and beamlet distribution produced by vertical slits

Figure 8-5: Longitudinal distribution of on-axis solenoid field

in $\mathrm{RF}$ gun

Figure 8-6: RMS beam envelope and projected RMS radial emittance

$8-10$

Figure 8-7: Geometry of skew quadrupole adapter beamline

$8-11$

Figure 8-8: Slice population and transverse

$8-12$

Figure 8-9: Longitudinal phase space at adapter entrance

$8-13$

Figure 8-10: The $y-z$ beam distribution at the adapter exit

$8-13$

Figure 8-11: Vertical-longitudinal beam distribution under energy mismatch conditions

Figure 8-12: Vertical emittance versus adapter mismatch energy.

Figure 9-1: TESLA 9-cell superconducting $1.3 \mathrm{GHz}$ cavity 9-3

Figure 9-2: TESLA cryomodule end view cross-section 9-4

Figure 9-3: Longterm variation of the resonant frequency of the eight cavities in a TTF cryomodule.

Figure 9-4: Resonant frequency variation of cavity \#1 and corresponding histogram

Figure 9-5: Microphonics in the TTF module $\quad 9-7$

Figure 9-6: Generator power requirement $9-9$

Figure 9-7: Generator power for four beam currents $\quad$ 9-10

Figure 10-1:. X-ray pulse duration 10-2

Figure 10-2: Electric and magnetic field distribution of a deflecting

dipole

$10-3$

Figure 10-3: Longitudinal electric field distribution of the deflecting mode in the 7-cell cavity at $3.9 \mathrm{Ghz}$

Figure 10-4: Electric field distribution of the monopole LOM

at $2.8581 \mathrm{Ghz}$

Figure 10-5:. Electric field distribution of the monopole LOM at $2.8685 \mathrm{Ghz}$

Figure 11-1:. Linac RF system schematic layout

Figure 13-1: A cross-section of the proposed cryogenic module

for the linac.

Figure 13-2: A Cross-section view of the increased diameter

TESLA cavity helium tank

Figure 13-3: Refrigeration system cost versus the diameter of the $1.9 \mathrm{~K}$ helium return

pipe

Figure 13-4: Simplified flow schematic diagram for the

cooling of and the cool down of the rf Cavities

Figure 13-5:

Figure 13-6: A flow schematic for a refrigerator cold box

and its warm and cold compressors

Figure 13-7: A flow schematic for the cold compressor end of a $1.9 \mathrm{~K}$ helium cold box

Figure 14-1: Magnets in a beam spreader region 
Figure 14-2: Septum Dipole Magnet

Figure 14-3: One quadrant of a septum quadrupole magnet in cross section

Figure 14-4: Plot of magnetic field lines for septum quadrupole

Figure 14-5: Magnet layout at the entrance to the ring arc sections

Figure 14-6: Starting geometry for bend magnet pole optimization

Figure 14-7: Dimensions of an optimized arc bend magnet pole.

Figure 14-8: Magnetic field plot for an arc dipole magnet after pole shape optimization

Figure 14-9:. Yoke and coil configuration of the quadrupoles

for the ALS booster

Figure 14-10: Cross-section of a lamination of the ALS sextupole design

Figure 14-11: Magnetic field plot of ALS 1/12 sextupole magnet

Figure 15-1: Comparison of fractional energy loss due to CSR

Figure 15-2: Beam loss fraction, due to Coulomb scattering

Figure 15-3: Conceptual layout of a representative section

of the vacuum system

Figure 15-4:

Figure 15-5: Pressure profile along a representative portion of the Arc 4 beam tube 
List of Tables 


\section{OVERVIEW OF THE FACILITY}

LBNL is pursuing design studies and the scientific program for a facility dedicated to the production of x-ray pulses with ultra-short time duration for application in dynamical studies of processes in physics, biology, and chemistry. The proposed x-ray facility has the short x-ray pulse length ( $\sim 60$ fs FWHM) necessary to study very fast dynamics, high flux (up to approximately $10^{11}$ photons/sec/0.1\% BW) to study weakly scattering systems, and tuneability over 1-12 keV photon energy. The hard $\mathrm{x}$-ray photon production section of the machine accomodates seven 2-m long undulators. This is the aspect of the machine that is the subject of the present report. Design studies for longer wavelength sources, using high-gain harmonic generation, are in progress, and will be the subject of future reports.

The $\mathrm{x}$-ray pulse repetition rate of $10 \mathrm{kHz}$ is matched to studies of dynamical processes (initiated by ultra-short laser pulses) that typically have a long recovery time or are not generally cyclic or reversible and need time to allow relaxation, replacement, or flow of the sample.

Synchronization of $\mathrm{x}$-ray pulses to sample excitation signals is expected to be approximately 50 - $100 \mathrm{fs}$. Techniques for making use of the recirculating geometry to provide beam-based seed and timing signals from early passes through the machine are being studied.

$\mathrm{X}$-rays in the $1-12 \mathrm{keV}$ photon energy range are produced by spontaneous emission in tuneable undulators, and will be linearly polarized. Figure 1-1 shows the photon flux (photons per second per $0.1 \%$ bandwidth) for the case of a $1 \mathrm{nC}$ bunch at $10 \mathrm{kHz}$ repetition rate, at 2.5 $\mathrm{GeV}$. Up to eleventh harmonic of undulator radiation is shown. Development of the $\mathrm{rf}$ photocathode gun to achieve higher charge per bunch will result in greater flux.

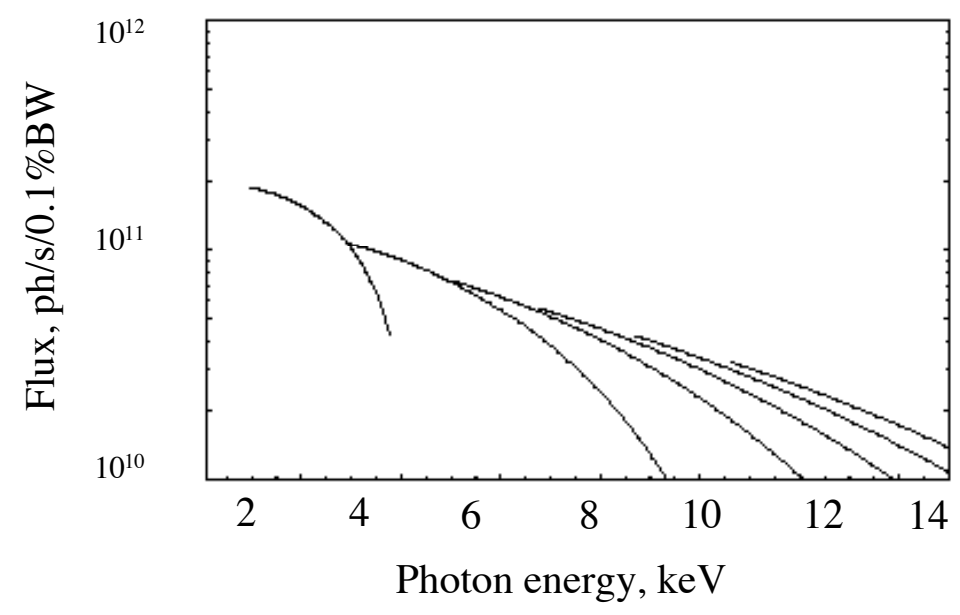

Figure 1-1 Average flux as a function of photon energy. 
The major components and systems of the machine are an rf photo-injector, a linear preaccelerator, a main linear accelerator, magnetic arcs and straight sections, deflecting cavities, a hard x-ray photon production section, and a beam dump [1]. The layout for this baseline configuration is shown in Figure 1-2.

Electron pulses of approximately $20 \mathrm{ps}$ duration and 1-3 $\mathrm{nC}$ charge are produced in a highbrightness rf photocathode gun and accelerated to $10 \mathrm{MeV}$. As described in Chapters 5-RF Photocathode Gun and 8-Flat Beam Adapter, application of a solenoidal magnetic field on the cathode, followed by a specially configured skew-quadrupole channel, allows production of a flat beam with $x / y$ emittance ratio $>50: 1$ and vertical normalized emittance less than $1 \mathrm{~mm}-\mathrm{mrad}$ [1]. This principle has been successfully demonstrated at the Fermilab/NICADD Photoinjector Laboratory (FNPL) [2], and LBNL has joined the experiment collaboration to help further work in this regard. Further details may be found in Chapter 7-Experimental Studies at FNPL.

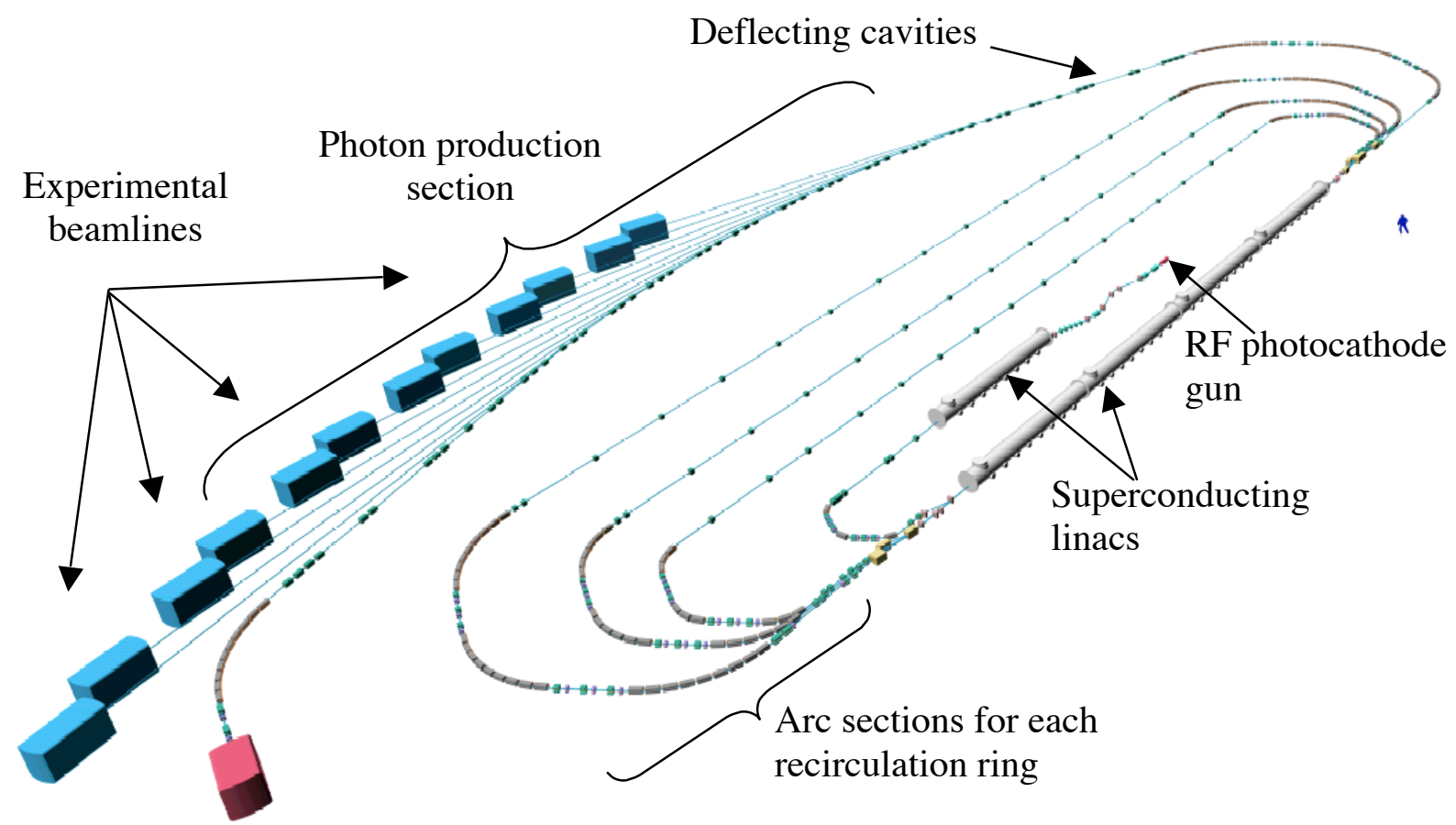

Figure 1-2. Machine layout for the baseline configuration. The beam generated at the rf photocathode gun travels through the injector linac, main linac, transport arcs, deflecting cavities, and photon production section, to the beam dump. The machine footprint is approximately $150 \mathrm{~m} \times 50 \mathrm{~m}$. Both undulator and bend magnet beamlines are shown here.

The electron bunches from the rf gun are further accelerated in a superconducting linear preaccelerator to $120 \mathrm{MeV}$. Following this injector linac, the beam passes through a higherharmonic accelerating structure, which is designed to condition the correlated energy spread in the bunch to prepare for compression from $20 \mathrm{ps}$ to $2 \mathrm{ps}$. After compression in a $180^{\circ}$ arc the beam is transported to the entrance of the recirculating linear accelerator. 
In the recirculating linac the final energy of $\sim 2.5 \mathrm{GeV}$ is achieved after four passes through the $600 \mathrm{MeV}, 1.3 \mathrm{GHz}$ superconducting rf structure. At the exit of the final arc the electron bunches receive a time-correlated vertical kick in a dipole-mode rf cavity. This imparts to the electron bunch a transverse momentum that is correlated in amplitude to longitudinal position within the bunch. The electrons then radiate $\mathrm{x}$-rays in the downstream chain of undulators and dipole magnets, imprinting this correlation in the geometrical distribution of the $x$-ray pulse, see Figure 1-3. The correlated x-ray pulse is then compressed by use of asymmetrically cut crystal optics to achieve the ultra-short photon pulse length as described in Chapter 18-Beamlines [3]

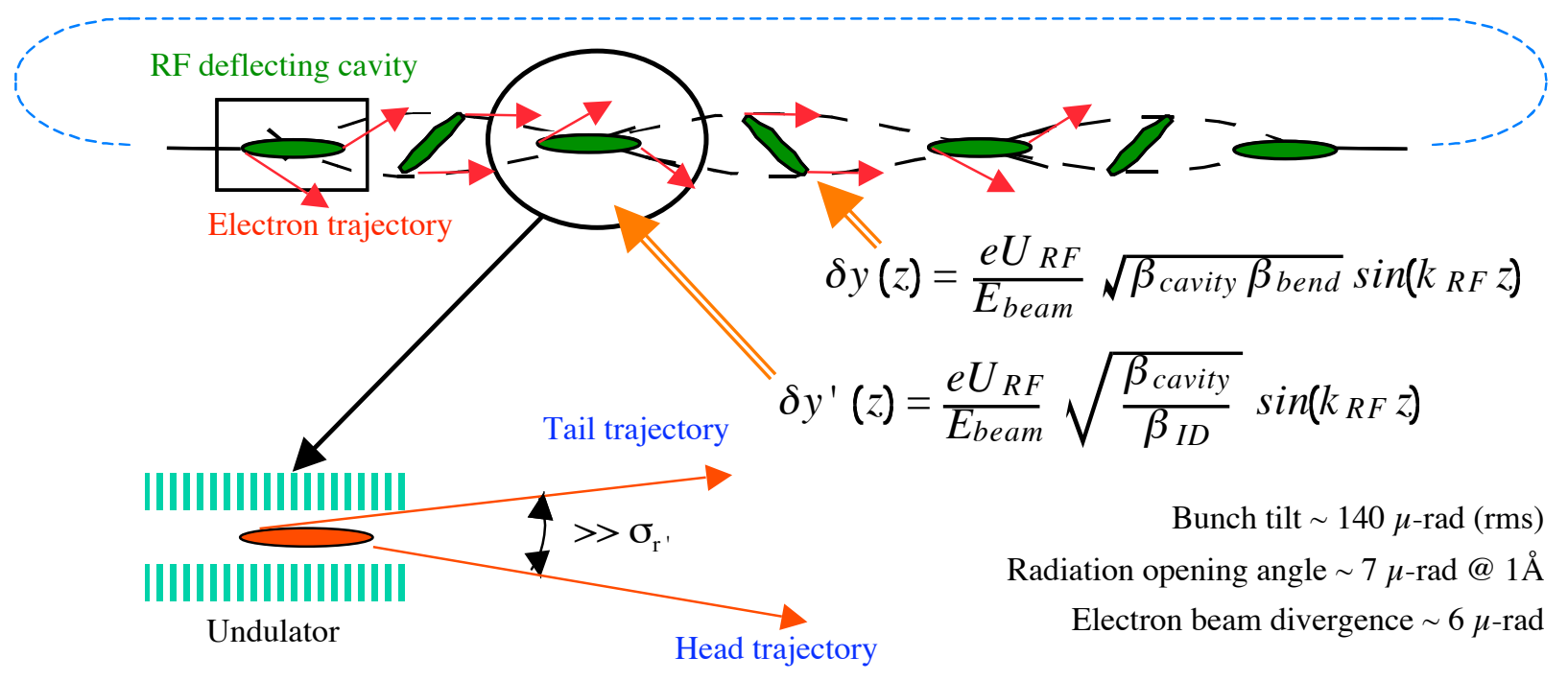

Figure 1-3. Time/position/angle correlation of the electron bunches. By providing divergence larger than the radiation opening angle, or beamsize greater than the diffraction limited size, optical elements can be used to compress the radiation from a bunch [3]. The resultant $x$-ray pulse length may then be limited by the electron bunch emittance.

The reason for producing a beam with large $x / y$ emittance ratio may now be seen. The electron bunch must be sheared by the action of the deflecting cavity, such that the time duration of a slice of the bunch is short compared to the total bunch length. Figure 1-4 illustrates the principle. 


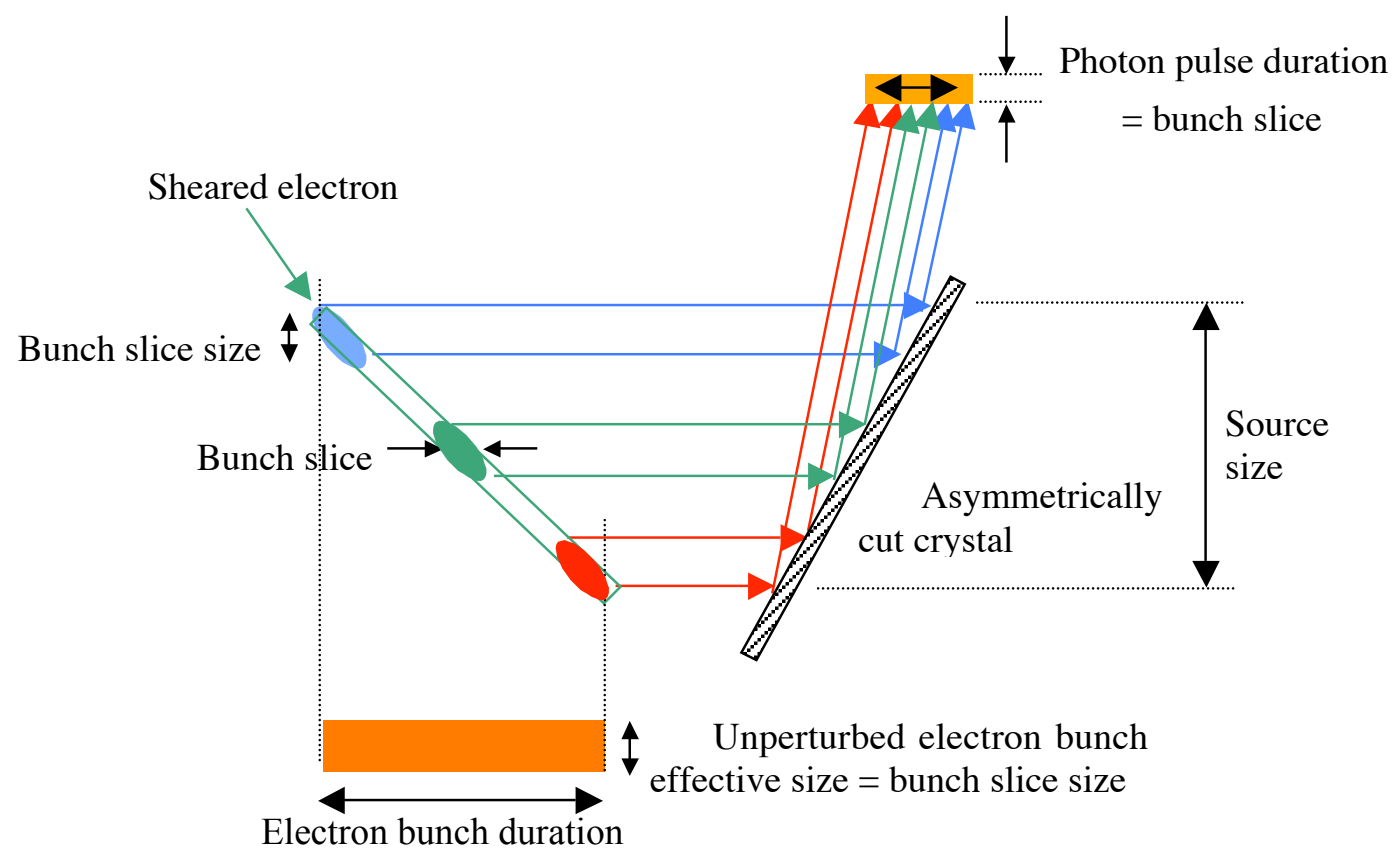

Figure 1-4 Compression of the $x$-ray pulse. The electron bunch, sheared following the transverse kick applied in the deflecting cavities, is represented as the source of $x$ rays, with three "slices" of the bunch indicated in different colors. Each slice has a longitudinal dimension determined by the unperturbed electron bunch transverse dimension, the diffraction-limited radiation source size, and the magnitude of the deflection of the bunch.

For significant deflection of the bunch, the x-ray pulse duration $\square_{x \text {-ray }}$ (or slice duration) may be written in terms of the electron bunch length $\square_{1}$, the source size presented by the perturbed bunch $\square_{\text {source }}$, and the unperturbed electron bunch effective size $\square_{\text {bunch effective }}$ :

$$
\frac{U \text { x-ray }}{\square \text { buncheffective }}=\tan \frac{\square l}{\square \text { source }} \square \frac{\square l}{\square \text { source }}
$$

The source size is described by the lattice optics and the deflecting cavity voltage $U_{R F}$

$$
\square_{\text {source }}=\frac{e U_{R F}}{E} \sin \left(k_{R F} \square_{l}\right) \sqrt{\square_{\text {cavity }} \square_{\text {source }}}
$$

The effective electron bunch size due to the beamsize and the diffraction limited image size is

$$
\square \text { buncheffective }=\sqrt{\square \text { beamsize }+\square_{\text {radiation }}^{2}}
$$

then the $\mathrm{x}$-ray pulse duration is expressed as

$$
\square_{x-\operatorname{ray}} \square \frac{E}{e U_{R F} k_{R F}} \frac{\square_{\text {beamsize }}}{\sqrt{\square_{\text {cavity }} \square_{\text {source }}}} \sqrt{1+\left(\frac{\square_{\text {radiation }}}{\square_{\text {beamsize }}}\right)^{2}}
$$


A similar expression may be derived for radiation from a diverging source. For short wavelengths, at a given deflecting voltage, the x-ray pulse length is limited by the electron beam emittance. For long wavelengths, the radiation diffraction limited source size dominates and the $\mathrm{x}$-ray pulse length is increased.

Figure 1-5 shows the dependence of $x$-ray pulse duration (plotted as full-width at half maximum) as a function of the $\mathrm{x}$-ray photon energy for three deflecting cavity voltages and baseline parameters (beta-function at the cavities $\square_{\mathrm{RF}}=90 \mathrm{~m}$ beta-function at the insertion devices $\square_{\text {undulator }}=2 \mathrm{~m}$, beam energy $\mathrm{E}=2.5 \mathrm{GeV}$, beam emittance $\square=9.8 \times 10^{-11} \mathrm{~m}$, and $\mathrm{rf}$ wavenumber $\mathrm{k}=82 \mathrm{~m}^{-1}$ ).

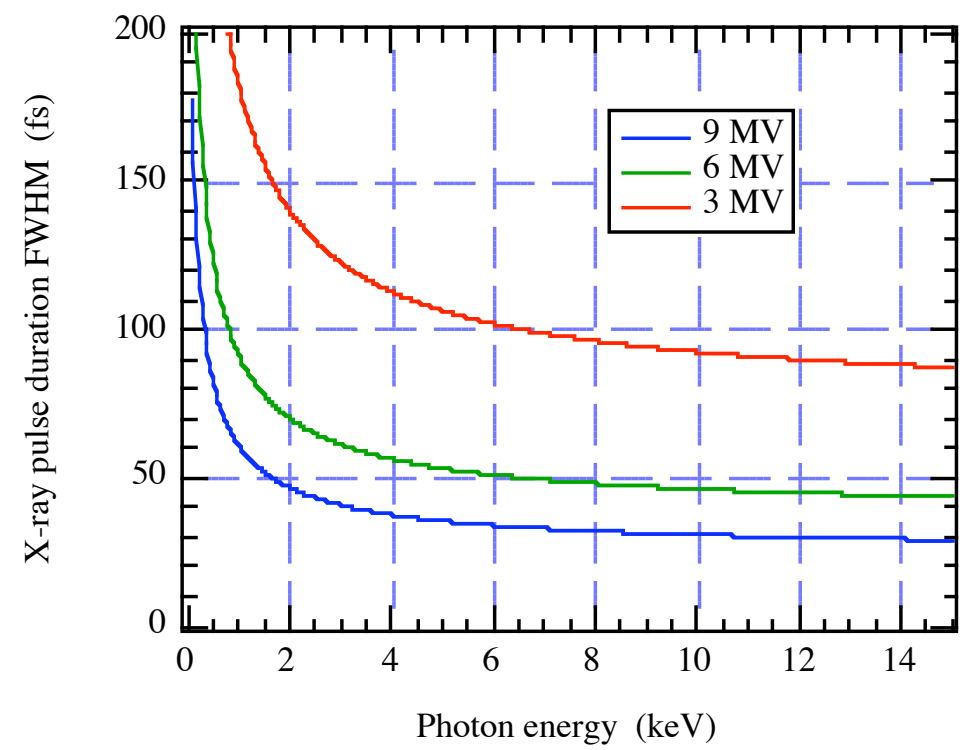

Figure 1-5 X-ray pulse length as a function of photon energy, for three values of deflecting if voltage.

For baseline operation at $25 \mathrm{~kW}$ beam power, the arc returning the $2.5 \mathrm{GeV}$ beam to the linac is not required, and the beam will be taken to a shielded dump as described in Chapter 20-Beam Dump. The option for energy recovery in the linacs be maintained for operations with increased beam power.

The machine lattice is described in Chapter 3-Accelerator Physics, and our studies demonstrate that the lattice can be made to preserve beam transverse and longitudinal emittance [4]. Emittance control and understanding and mitigation of collective effects is critical to a successful machine design, and we are addressing key aspects of accelerator physics involved in beam break-up coherent synchrotron radiation, the influence of resistive wall wakefields, and other effects as described in Chapter 4-Collective Effects [5]. To preserve the six-dimensional emittance through the machine, high-quality instrumentation and diagnostics systems will be required. Techniques for measurement of beam parameters are described in Chapter 16-Beam Diagnostics. 
Longitudinal dynamics have been modeled from the rf gun through the injector linac and all passes of the main linac, and emittance dilution due to non-linearities in the rf waveform have been assessed. In the injector, harmonic cavities will be used to linearize the longitudinal phasespace. Chapter 3-Accelerator Physics describes this manipulation of longitudinal phase-space. The bunch lengths and beam pipe apertures under current consideration result in a regime in which the coherent synchrotron radiation impedance may be significant in the lowest energy arcs of the machine, and studies of such effects are reported in Chapter 4-Collective Effects. A smaller beam pipe aperture is beneficial in shielding coherent synchrotron radiation, but the minimum aperture appears to be limited by transverse wakefields arising from the resistive wall impedance.

Conventional photocathode rf guns employ a half-length pillbox cell for the cathode cavity followed by a full cell for rapid acceleration of emitted electrons, and operate in the $10 \mathrm{~Hz}$ pulse repetition frequency range. For $\mathrm{CW}$ or high duty factor operation, thermal limitations will likely prevent such a design from operating at sufficiently high gradient. We have produced a conceptual design with optimized cavity geometry to allow cooling of the cavity surfaces, and operation at high gradient and high repetition rate [6]. In this design the first cell is modified by the inclusion of a re-entrant nose-cone, on the end face of which the photo-cathode is mounted. This nose-cone serves two purposes: it increases surface area to reduce deposited power density and it enhances the accelerating electric field at the cathode. The design is further described in Chapter 5-RF Photocathode Gun.

The high-brightness electron source must be carefully optimized to minimize and preserve the beam emittance, particularly against deleterious effects of space-charge. We have begun optimization of electron launch phase, rf cavity conditions, and magnetic focussing channels to produce a low-emittance beam. Several simulation codes have been used in this study, as described in Chapter 6-Beam Dynamics in the rf Gun.

Our linac design is based on superconducting $\mathrm{rf}$ technology developed for the TESLA project [7]. Identical cryomodules are used for the main linac and the injector linac. Our design considerations here are for a peak accelerating gradient of $20 \mathrm{MV} / \mathrm{m}$ in the main linac. The pulse repetition time of $100 \square \mathrm{s}$ is less than the superconducting cavity filling time, and the linacs must operate in $\mathrm{CW}$ mode. The rf power requirement for the linacs is dominated by the need to overcouple to provide bandwidth necessary for feedback systems to maintain phase and amplitude control against cavity detuning by microphonics. Approximately $8 \mathrm{~kW}$ rf power is required per cavity, assuming a bandwidth of $40 \mathrm{~Hz}$. The total linac rf power requirement is approximately $300 \mathrm{~kW}$. Chapter 9-Superconducting RF Linacs describes the design considerations, and Chapter 11-Linac RF Systems describes the power requirements.

The heat dissipated in the power coupler and the cavity helium bath is approximately 100 times greater than for TESLA operations, however an analysis indicates that with additional connections from the supply line to the helium bath for each cavity we may operate at these levels, Chapter 13-Cryogenics describes the refrigeration and cryogenics transport systems.

$3.9 \mathrm{GHz}$ superconducting cavities provide the deflecting voltage along the bunch, and $\mathrm{rf}$ designs for 7-cell cavities operating in a hybrid TM/TE mode are described in Chapter 10Deflecting Cavities. Seven such cavities will be required to provide a deflecting voltage of up to 8.5 MV, allowing for operations at the higher beam energy of $3.1 \mathrm{GeV}[8]$. 
Several narrow-gap in-vacuo superconducting undulator designs have been characterized as high-flux sources and are summarized in Table 1-1. The machine design accommodates an energy increase to $3.1 \mathrm{GeV}$, and in that case the flux of $10 \mathrm{keV}$ photons from $1 \mathrm{nC}$ bunches at $10 \mathrm{kHz}$ may be increased to $5.6 \times 10^{10}$ photons $/ \mathrm{sec} / 0.1 \% \mathrm{BW}$. We also envisage development of photocathode gun performance to reach $3 \mathrm{nC}$ per bunch, resulting in $\sim 10^{7}$ photons/pulse at $10 \mathrm{keV}$.

Synchronization and timing of the $\sim 60$ fs $x$-ray pulse to the experimental excitation pulse is critical to studies of ultra-fast dynamics. For our scheme of bunch manipulation followed by $\mathrm{x}-$ ray pulse compression we find that the phase jitter of the deflecting cavities with respect to the experimental laser pulse dominates timing issues [9]. We propose to derive all accelerator $\mathrm{rf}$ signals from phase-locked laser oscillators. The rf gun, linacs, and deflecting cavities are thus phase-locked to the experimental pump lasers, and timing jitter between the optical laser and the $\mathrm{x}$-ray pulse emitted by the beam is minimized. Phase and amplitude feedback of the deflecting cavities is expected to provide x-ray pulse to laser pulse stability of better than $100 \mathrm{fs}$. These techniques are described in Chapter 19-Synchronization.

\section{Table 1-1 Average flux for three in-vacuo undulator designs, $1 \mathrm{nC}$ bunches at $10 \mathrm{kHz}$ rate}

\begin{tabular}{|c|c|c|c|c|c|}
\hline Period & Gap & $\begin{array}{l}\text { Peak magnetic } \\
\text { field }\end{array}$ & $\mathrm{Kmax}$ & $\begin{array}{c}\text { Flux at } 2 \mathrm{keV} \\
2.5 / 3.1 \mathrm{GeV}\end{array}$ & $\begin{array}{c}\text { Flux at } 10 \mathrm{keV} \\
2.5 / 3.1 \mathrm{GeV}\end{array}$ \\
\hline $\mathrm{mm}$ & $\mathrm{mm}$ & $\mathrm{T}$ & & \multicolumn{2}{|c|}{$10^{10}$ photons $/ \mathrm{sec} / 0.1 \% \mathrm{BW}$} \\
\hline 20 & 5 & 1.5 & 2.8 & $8 / 11.4$ & $1.1 / 2.7$ \\
\hline 14 & 5 & 1.5 & 2.0 & $15.6 / 17.8$ & $2.3 / 4.5$ \\
\hline 14 & 3 & 2.0 & 2.6 & $15.6 / 17.8$ & $3.4 / 5.6$ \\
\hline
\end{tabular}

Laser systems will be an integral part of the machine, providing stable timing and synchronization signals, as well as the electron source through the photocathode laser, and the experimental pump lasers. Laser systems are described in Chapter 17.

Lattice magnets are of conventional water-cooled electromagnet design, with the exception of a few specialized magnets in the beam spreader and combiner regions adjacent to the main linac. In these compact regions special septum magnets are employed, dipoles which act on beams of differing energy, and small quadrupole designs in order to act on separate orbits of beams at different energies. Chapter 14-Magnets describes the designs.

The vacuum systems are less demanding than typical storage rings due to the much reduced outgassing from a relatively small average beam current, and the modest lifetime requirements of a pulsed machine. Chapter 15-Vaccum Systems presents an analysis of requirements.

Conventional facility requirements for the proposed facility are presented in Chapter 21. Issues in selecting a local site for the facility are discussed in Chapter 22-Site Selection.

Chapter 2 describes trade studies made to arrive at the baseline configuration presented here. Chapter 23 presents an analysis of the most significant risk factors. 
Table 1-2 Major baseline design parameters

\begin{tabular}{|l|l|}
\hline Bunch charge & $1 \mathrm{nC}$ (goal 3 nC) \\
\hline Bunch repetition rate & $10^{4} \mathrm{~Hz}$ \\
\hline Beam energy in photon production section & $2.5 \mathrm{GeV}$ \\
\hline Beam energy spread at $2.5 \mathrm{GeV}$ & $200 \mathrm{keV}$ \\
\hline Bunch length in photon production section & $2 \mathrm{ps}$ (full length) \\
\hline Electron tilt angle in the undulator & $0.14 \mathrm{mrad}$ \\
\hline RF deflection cavity frequency & $3.9 \mathrm{GHz}$ \\
\hline RF deflection cavity transverse voltage & $8.5 \mathrm{MV}$ \\
\hline Undulator length & $2 \mathrm{~m}$ \\
\hline Bunch length at gun exit & $20 \mathrm{ps}$ \\
\hline Normalized emittance from gun & $3 \times 10^{-6} \mathrm{~m}-\mathrm{rad}$ \\
\hline $\begin{array}{l}x / y \text { normalized emittance from flat-beam } \\
\text { transformer }\end{array}$ & $20 / 0.4 \times 10^{-6} \mathrm{~m}-\mathrm{rad}$ \\
\hline Beam energy spread from gun & $\pm 20 \mathrm{keV}$ \\
\hline Energy gain per pass in main linac & $600 \mathrm{MeV}$ \\
\hline
\end{tabular}

The configuration described in the present report is concerned primarily with the production of hard x-ray's. In addition, conceptual design studies for producing high-flux, short-pulse photons at lower photon energies are in progress. Of particular interest is the technique of highgain harmonic generation [10]. The scheme is shown in Figure 1-6. A high-brightness electron beam is accelerated to $\sim 2.5 \mathrm{GeV}$, and passed through an undulator where a seed laser modulates the charge over a short length of the bunch. This modulation is then amplified in a following undulator, tuned to a higher harmonic of the seed laser. The electron pulse may then be delayed in a short chicane, and the process repeated by modulating the beam this time with the harmonic radiation produced in the previous undulator.

This technique allows variable flux, up to $10^{12}$ photons per pulse, and possibly up to $\mathrm{keV}$ photon energies. Details of this scheme will be reported in future publications. 

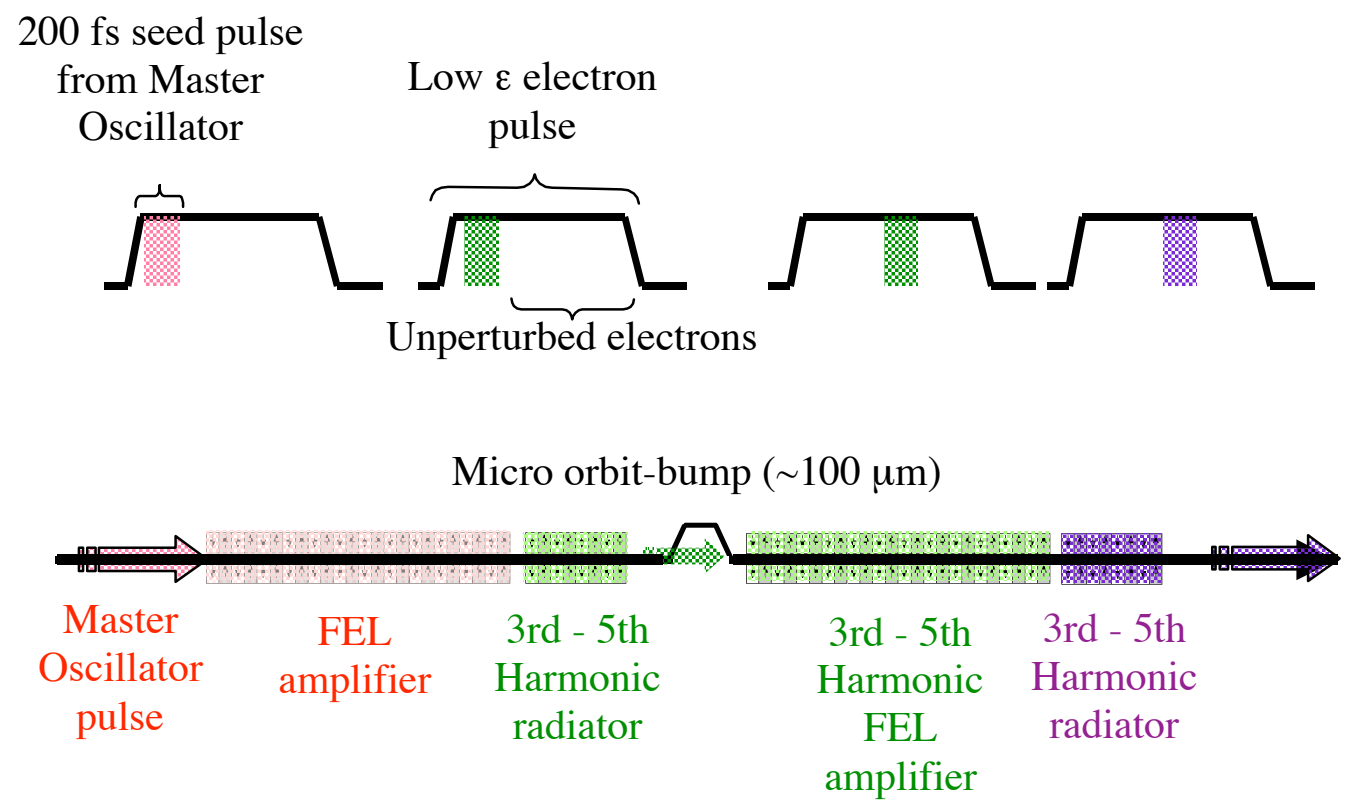

Figure 1-6 UV and soft x-ray production technique using high-gain harmonic generation.

\section{REFERENCES}

[1] J.N. Corlett, S. DeSantis, N. Hartman, P. Heimann, R. LaFever, D. Li, H. Padmore, R. Rimmer, K. Robinson, R. Schoenlein, J. Tanabe, S. Wang and A. Zholents (LBNL), and D. Kairan (BINP) "Initial Feasibility Study of Dedicated Synchrotron Radiation Light Source for Ultrafast X-Ray Science” - LBNL 48171, October, 2001.

[2] D. Edwards et al, "The Flat Beam Experiment at the FNAL Photoinjector", Proc. XXth International Linac Conference, Monterey, 2000.

[3] A. Zholents et al "Generation of subpicosecond x-ray pulses using RF orbit deflection", NIM A 425 (1999) 385-389.

[4] A. Zholents et al, "Initial Lattice Studies for the Berkeley Femtosecond X-ray Light Source", Proc. $8^{\text {th }}$ European Particle Accelerator Conference, Paris, June 3-7, 2002.

[5] S. De Santis, A. Zholents, "Beam Break Up analysis for the Berkeley Femtosource", Proc. $8^{\text {th }}$ European Particle Accelerator Conference, Paris, June 3-7, 2002.

[6] R. Rimmer et al, "A High-Gradient High Duty-Factor RF Photocathode Electron Gun", Proc. $8^{\text {th }}$ European Particle Accelerator Conference, Paris, June 3-7, 2002.

[7] TESLA Technical Design Report, DESY 2001-011, March 2001.

[8] D. Li, J. Corlett, "RF Deflecting Cavity Design for the Berkeley Ultrafast X-ray Source", Proc. $8^{\text {th }}$ European Particle Accelerator Conference, Paris, June 3-7, 2002.

[9] J. Corlett et al, "Synchronization of X-ray Pulses to the Pump Laser in an Ultrafast X-ray Facility", Proc. $8^{\text {th }}$ European Particle Accelerator Conference, Paris, June 3-7, 2002.

[10] L.-H. Yu et al, "High-Gain Harmonic-Generation Free-Electron Laser", Science 289: 932934, 2000. 


\section{BASELINE CONFIGURATION CHOICES}

\section{REQUIREMENTS DRIVEN DESIGN}

During this pre-conceptual phase, various concepts have been explored and solutions developed with a focus on science and user-needs, costs, and technical risks. In this feasibility study we limit ourselves to presenting the trade studies and risk mitigation plans that most heavily bear on the hard x-ray production. Techniques for producing soft x-rays from cascaded harmonic generation in free-electron lasers are in development and will be described in future reports. The flexible configuration of the recirculating linac simultaneously accommodates several hard and soft x-ray production techniques in parallel.

The facility is designed to provide world-class capabilities in ultra-fast dynamics studies based predominantly on pump-probe experiments. The facility requirements were systematically derived as depicted in Figure 2-1, leading to the key performance parameters listed in Table 2-1. Note that this study is concerned with accelerator design optimized for hard x-ray production UV and soft x-ray schemes are under development and will be reported in the future.

Table 2-1 Key parameters for an ultrafast $x$-ray science user facility driven by scientific needs in physics, chemistry and biology.

Multi-user facility: Multiple experimental beamlines

Repetition rate: $\quad 10 \mathrm{kHz}$

Synchronization: better than $50 \mathrm{fs}$

Broad photon range: $\quad 0.02-10 \mathrm{keV}$

- Hard x-rays

- Tunable: 1 - $10 \mathrm{keV}$

- Pulse length: $\quad<100 \mathrm{fs}$

- Flux: $\quad 3 \times 10^{6}(\mathrm{ph} / \mathrm{pulse} / 0.1 \% \mathrm{BW}) @ 1 \mathrm{nC}$

- Soft x-rays and VUV radiation

- Tunable: $\sim 20-1000 \mathrm{eV}$

- Pulse length: 50 fs to 2 ps

- Flux: up to $1012(\mathrm{ph} / \mathrm{pulse} / 0.1 \% \mathrm{BW})$

- Lasers with temporal and spatial pulse shaping

- Tunable $267 \mathrm{~nm}-3000 \mathrm{~nm}$

- Polarization

- Switchable LH, RH circular polarization

- Flux stability

- $10-20 \%$ shot-shot variation, average over $\sim 10,000$ shots/second 


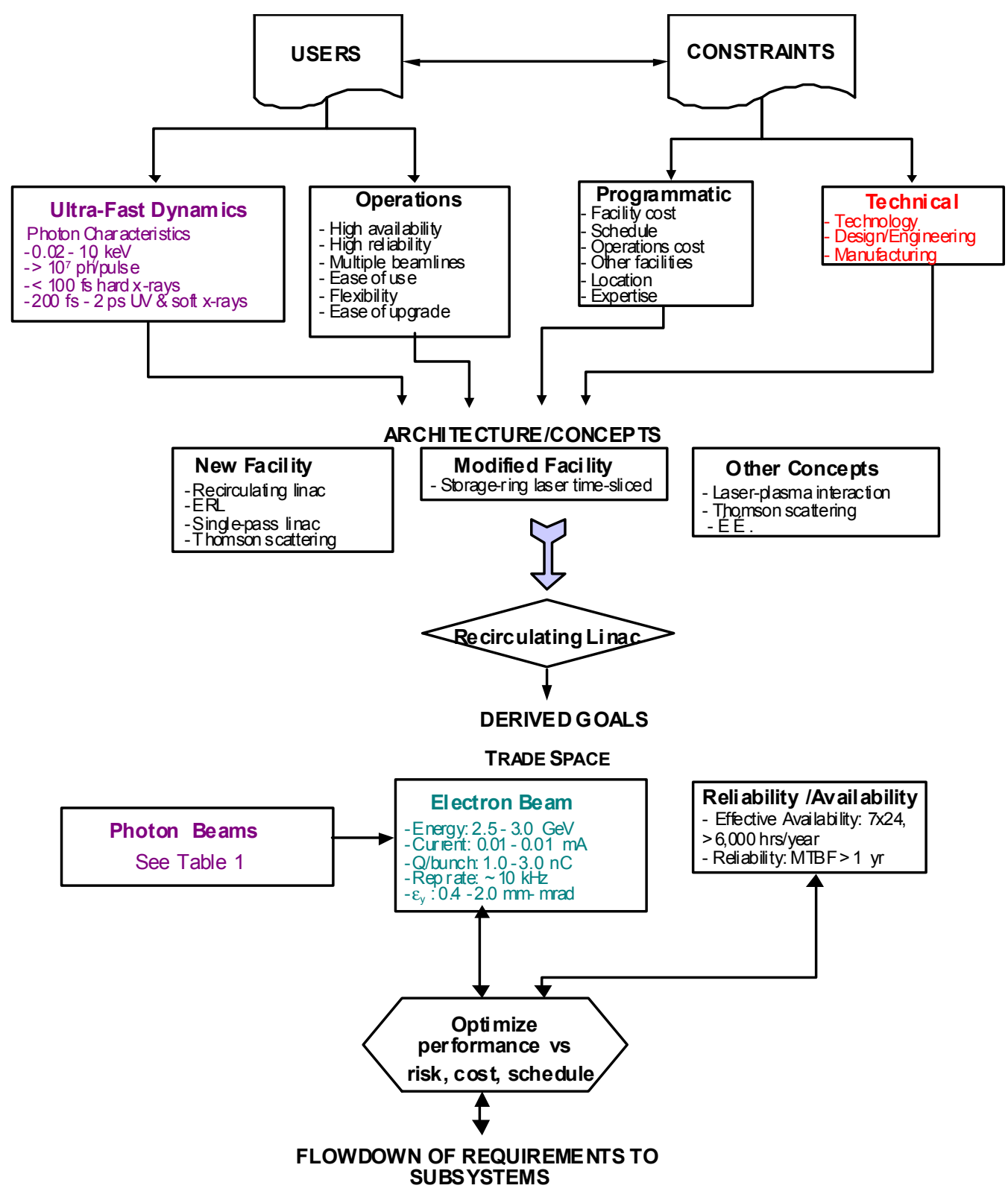

Figure 2-1 Derivation of systems requirements

\section{CONCEPT SELECTION}

The choice of a machine concept has a major impact on its development and performance. Several different options have been considered, including other facilities that are being proposed for operation in the same time frame. The proposed technologies may be categorized as follows:

1. Upgrades of existing facilities - These include implementing laser-slicing in Berkeley Advanced Light Source (ALS) beamlines, the Swiss Light Source (SLS) and others [1]. 
2. Single-Pass Energy Recovery Linacs (ERL) - These include the Cornell ERL, the Brookhaven PERL, and Daresbury 4GLS. The radiation is produced following a single pass through the linac, and the kinetic energy of the beam is recovered by returning through the linac [2-4].

3. Recirculating linacs - These include the Novosibirsk MARS, and the LBNL proposal. The basic concept involves multiple passes through the same linac to achieve the final beam energy [5].

4. X-ray Free Electron Lasers (FEL) - These include the SLAC LCLS, DESY Tesla XFEL, and the BESSY FEL. They utilize the beam from a single-pass linac to produce highly coherent and intense radiation [6-8].

5. Other concepts - These include x-ray generation from a laser-plasma interaction, Thomson scattering of laser photons from a relativistic electron beam, etc.

\section{Generation of Femtosecond Hard X-Ray Pulses}

The concept of ultra-short radiation pulse production by electron beam manipulation followed by x-ray pulse compression as proposed in Ref. [9] has been developed in this study. The physics for this concept has been described previously in this report, here we note that the photon pulse duration is a function of:

- Square-root of the vertical beam emittance, $\varepsilon_{\mathrm{y}}$

- RF deflection voltage, U

- Inverse of the square-root of deflecting cavity $\beta$-function, $\beta_{\mathrm{RF}}$

- Inverse of the rf wave number (frequency), $k_{\mathrm{RF}}$

- The lattice $\beta$-function at the radiation point, $\beta_{\mathrm{ID}}$ (dependent on location of radiation source)

- The radiation opening angle $\sigma_{\mathrm{r}^{\prime}}$ or size $\sigma_{\mathrm{r}}$

The implications for the design of the facility are the following:

- A photoinjector that produces a beam with a small emittance

- Flat beam optics in the photo-injector gun that generate a high $x / y$ emittance ratio with a resultant very small emittance in one (vertical) direction

- A linac, transport arcs, and lattice that preserve the emittances

- Deflecting cavities that provide a high peak voltage at high frequency

- A photon-production section lattice that allows a large variation in $\beta$-functions

- An undulator sufficiently long to produce small divergence in radiation, and a small (diffraction limited) angular divergence

For the hard x-ray applications of this facility, experiments requiring $\mathrm{x}$-ray flux in short pulses are favored over those experiments requiring brightness. In this $1-10 \mathrm{keV}$ photon energy range, brightness is traded for short pulse duration. The photon flux on a sample at the end of a beamline is derived from all electrons in a bunch, and so produces a significant increase of $\sim 10^{4}$ times what is achievable by the time-slicing method currently being pursued at the ALS and other storage rings.

Another potential technique, which also takes advantage of the correlated electron positions within a bunch, may be to use position-sensitive detectors and the angle-time or coordinate-time 
correlation of the radiation to simultaneously observe photons with different time delays. This technique would not involve direct compression of the photon pulse.
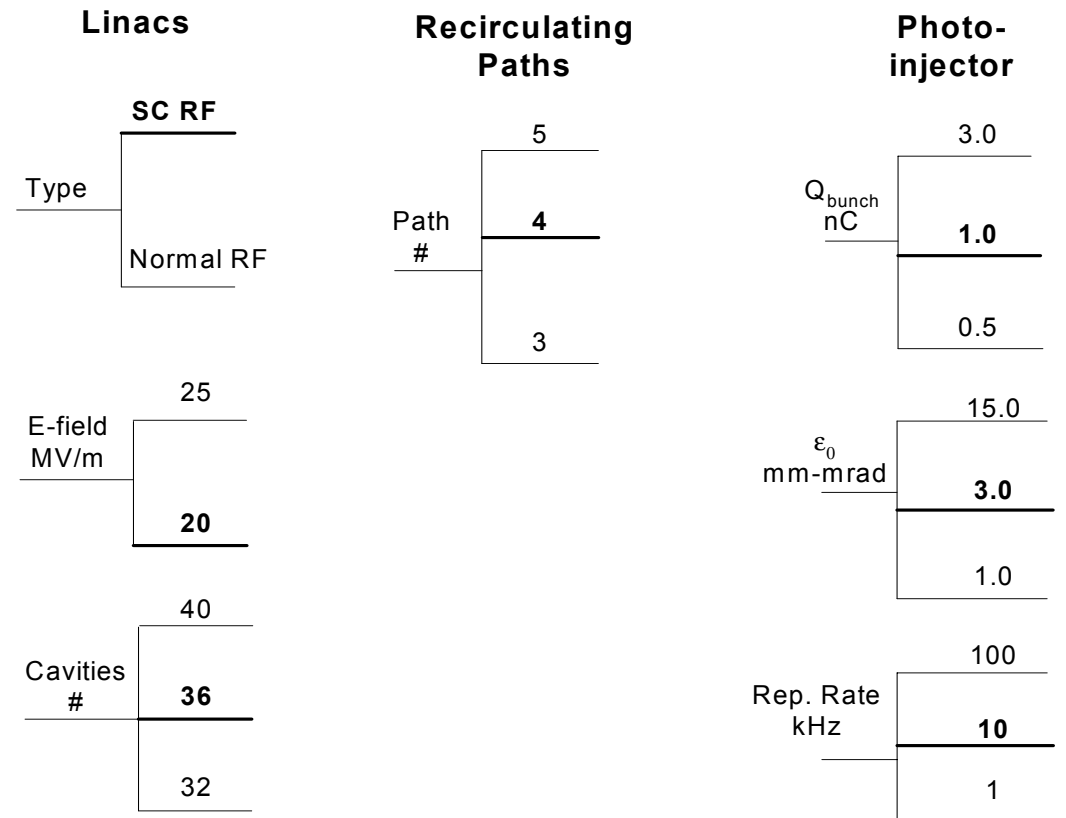

Deflecting cavities
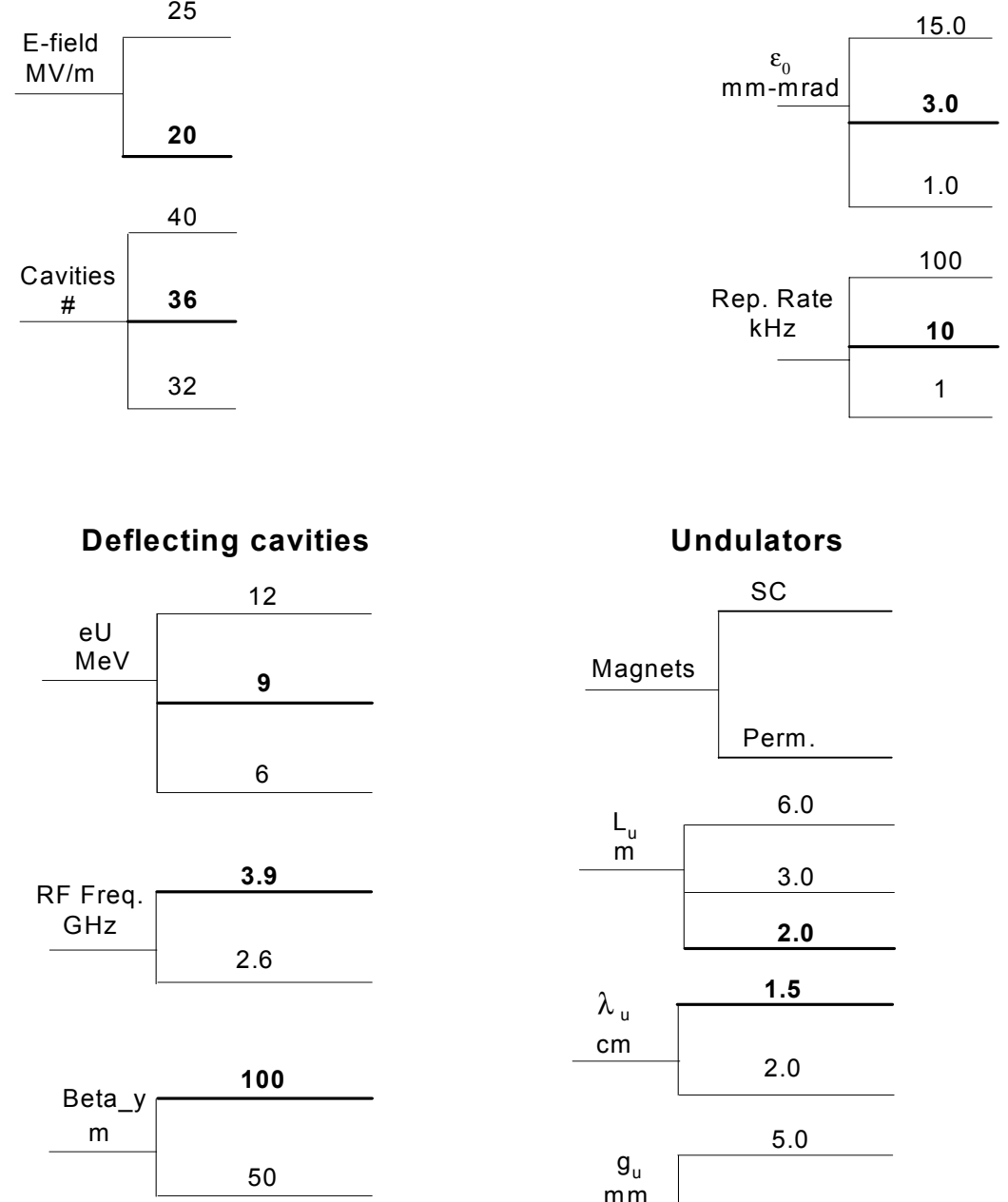

Undulators
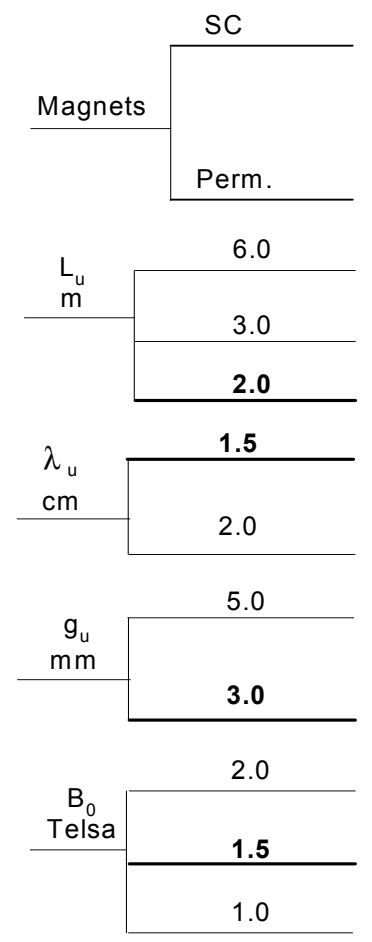

Flat beam optics

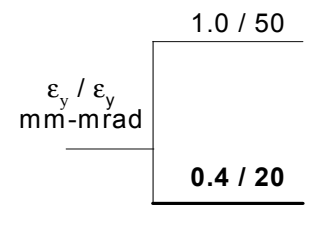

Figure 2-2 Trade-space for baselining the recirculating linac hard $x$-ray configuration. 


\section{DeVelopment OF THE BASEline CONFiguration}

A staged low-risk approach has been adopted, in which the baseline design described here incorporates flexibility to allow for (i) upgrades to higher beam energies and/or bunch repetition rate; (ii) possible energy recovery; and (iii) additional insertion devices including free electron lasers (FEL's) as dictated by user needs.

This feasibility study is limited to presenting the trade studies and risk mitigation plans that most heavily bear on the production of hard x-rays. This baseline design is for hard x-ray production, and addition of VUV and soft x-ray components will be addressed in the future.

The facility requires accelerator technology in many areas that has been demonstrated on successful operating machines, but presents some needs for technology development.

Recirculating linac designs have been investigated for different configurations of photoinjectors, linacs, undulators, etc, as illustrated in the trade-space represented in Figure 2-2. The heavy lines and bold text represent the present baseline. Some trades studies are still to be completed and further analyses will be performed as the design and technologies evolve. Several key trade studies are summarized below.

\section{Choice of Accelerator RF Technology}

Both superconducting and normal conducting rf technologies have been considered for the injector and main linacs. Normal conducting rf linac technology has a proven track record and the fabrication is mature. Superconducting cavities have been demonstrated to achieve accelerating gradients exceeding $25 \mathrm{MV} / \mathrm{m}$ at $\mathrm{Q}_{0}$ over $10^{10}[10]$. A normal conducting linac requires $\sim 10^{6}$ times more $\mathrm{rf}$ power for the same acceleration gradient than the superconducting linac, but $Q$-values are sufficiently low to allow pulsed operation at $10 \mathrm{kHz}$ repetition rate. Maintaining stability of the multiple pulsed rf systems then introduces problems in producing good beam energy and phase stability. In order to facilitate a bunch rate of $10 \mathrm{kHz}, \mathrm{a}$ superconducting linac must operate in a Continuous Wave $(\mathrm{CW})$ mode, while the state-of-the-art TESLA cavities and cryomodules are designed for a low duty factor of approximately $0.7 \%$. Superconducting rf operating in $\mathrm{CW}$ mode has advantages of allowing better stabilization of beam energy and phase (timing jitter), and requires significantly reduced rf power systems. The disadvantage is in the requirement for a significant cryogenics system.

Considering issues such as pulse-to-pulse stability (essential to achieve synchronization requirements), rf power requirement, future upgrades, state of technology, availability, and beam dynamics associated with wakefield effects, a superconducting linac has been selected as the baseline for the machine. Further details are found in Chapter 8-Superconducting RF.

The number of cavities needed to achieve an energy gain of greater than $600 \mathrm{MeV}$ per pass depends on the accelerating gradient and the cavity design. For example, the TESLA FEL design uses 9-cell cavities and integrates eight cavities into a single cryomodule. Optimization of the design of the cryomodules is proceeding in parallel with the refrigeration systems design. Details are presented in Chapter 12-Cryogenics. 


\section{Recirculating Passes}

The number of recirculating paths influences the design, cost, and operation of the facility. As the number of passes increases for a given ultimate beam energy, the cost of the rf subsystem decreases while the cost of the beam transport subsystem increases. The configuration has been designed to minimize project cost for a 2.5 to $3.1 \mathrm{GeV}$ electron beam. Cost estimates at this early stage have inherently high uncertainty and should be treated as

Rough Order of Magnitude (ROM) costs. Figure 2-3 provides the relative dependence of the cost of the relevant subsystems and their sum on the number of passes.

Figure 2-4 shows that the four-pass and five-pass designs achieve the lowest costs, although the minimum is quite shallow. Of particular consideration in selecting either four of five passes as the optimum, we note that design and operations increase in complexity with the number of passes. Based on these results, considerations, and cost uncertainties, the four-pass configuration has been selected as the baseline. The footprint of the baseline machine is approximately $150 \mathrm{x}$ $50 \mathrm{~m}$.

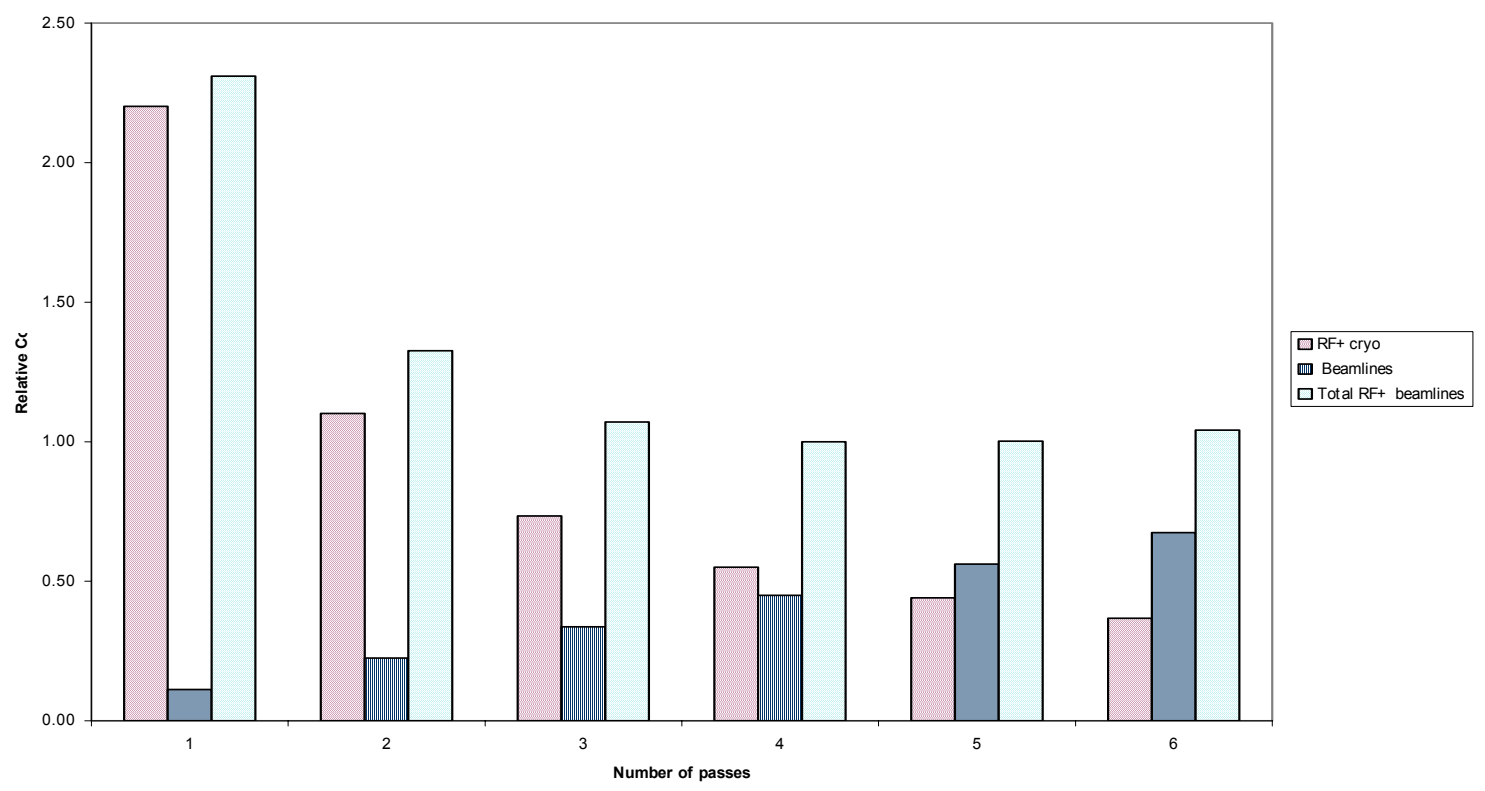

Figure 2-3 Recirculating linac cost trend versus the number of passes

\section{Photoinjector}

The photoinjector is probably the most challenging and critical component of the facility. The science needs for a large photon flux in a short pulse drive the photoinjector specifications that include:

- High charge per bunch of initially $1 \mathrm{nC}$, with a goal of $3 \mathrm{nC}$

- Small normalized emittance from the rf gun, $\varepsilon_{0} \sim 3 \mathrm{~mm}$-rad, to readily support a flat beam with a low normalized vertical emittance, $\varepsilon_{\mathrm{y}} \sim 0.4 \mathrm{~mm}-\mathrm{rad}$

- High repetition rate, $10 \mathrm{kHz}$ 
In addition, to support the reliability and availability demanded of a user-facility, the photoinjector is required to be highly stable in operations and an have operational life of several months. The components of the photoinjector, i.e. the drive laser, photocathode, and rf cavities, are critical elements to achieve these specifications.

The emittance of the beam is a critical accelerator physics parameter that defines the quality of the beam leaving the rf photocathode gun. We note the following characteristics:

- Surface physics and chemistry effects give rise to a distribution in velocities of the electrons as they are emitted from the cathode surface, resulting in the "thermal emittance"

- The emittance increases in a complex manner with the charge per bunch, $Q_{b}$ due to spacecharge effects at low energies

- Space charge effects and resultant emittance growth may be reduced by increasing the gradient of the accelerating electric field near the cathode surface. A larger field accelerates the electrons rapidly and reduces the effects from space-charge forces

There is presently some uncertainty in the reliably obtainable emittance as a function of bunch charge. Our assessment is that space charge effects can be significantly improved through additional R\&D and such experiments are being actively pursued.

Emittance growth due to space charge may be controlled by producing a long electron bunch ( $\sim 20 \mathrm{ps})$ at the cathode and accelerating rapidly in a high electric field rf gun. Physics and engineering studies indicate that $\mathrm{rf}$ cavities providing the required large accelerating fields at a high repetition rate may be fabricated using conventional copper construction and cooling techniques [see Chapter 5-RF Photocathode Gun].

The specified emittance of $3 \mathrm{~mm}$-mrad from the rf photocathode gun is close to that achieved at the exit of the Fermilab A0 gun [11]. An emittance increase of perhaps even a factor of five may be acceptable since the x-ray pulse duration scales as the square-root of emittance. Even in such circumstances, the pulse length remains below 100 fs at $10 \mathrm{keV}$. Such a trade may be particularly valuable for larger bunch charge where one gains appreciably in flux.

The choice of photocathode material is influenced by thermal emittance, quantum efficiency, availability of laser systems with wavelength matching the cathode work function, reliability, lifetime, and ease of fabrication. The operational lifetime depends on the rate of degradation of the photocathode QE rather than simply its initial value. Experience indicates that adequate lifetimes for a user facility will be achieved through controlled manufacturing techniques and good vacuum and thermal designs.

The baseline design is for a relatively small current of $10 \mu \mathrm{A}$. Commercially available lasers have been identified that can support currently viable photocathode options, with some additional requirements in pulse shaping prior to the cathode. Nevertheless, a photocathode with a high quantum efficiency is specified to limit the power and control the cost of the photocathode laser, and reduce the heating of the cathode. Based on the above considerations and the data presented in Chapter 5-RF Photocathode Gun, $\mathrm{Cs}_{2} \mathrm{Te}$ has been selected as the baseline photocathode material. 


\section{Flat Beam Optics}

As described in Chapter 7-Experimental Studies at FNPL the production of electron pulses of $\sim 20$ ps duration with $x: y$ emittance ratio $\sim 50: 1$ and with the smaller normalized emittance of less than $1 \mathrm{~mm}$-mrad has been successfully demonstrated at $1 \mathrm{nC}$ bunch charge [12]. Our requirements are within factor of 2 to 3 for emittance and charge of these values, and our baseline design uses the same technique for the production of a flat beam. The approach is to apply a solenoidal magnetic field on the cathode followed by a specially configured skewquadrupole channel to manipulate the angular momenta of the electrons, resulting in a highly asymmetric transverse emmittance configuration. Further details and investigations into different configurations to minimize the vertical emittance as described in Chapter 8-Flat Beam Adapter.

\section{Deflecting Cavities}

To provide position-angle correlation within a bunch, a dipole-mode deflecting cavity operating at a frequency of $3.9 \mathrm{GHz}$ is used, with a transverse voltage of $\sim 8.5 \mathrm{MV}$. Designs with the following objectives have been investigated:

- Take advantage of existing deflecting cavity designs, e.g. developed by FNAL and Cornell University

- Optimize the cavity geometry to minimize the rf power requirements

- Reduce short-range wake-field effects

- Increase the efficiency by operating at higher frequencies

Based on these studies, a 7-cell 3.9 GHz cavity has been selected. We need seven such cavities to obtain a voltage of $8.5 \mathrm{MV}$, details are presented in Chapter 10-Deflecting Cavities. We may trade this deflecting voltage and frequency with the vertical beta-function in the deflecting rf structure since increasing its value also benefits shorter x-ray pulses.

\section{Undulators}

The baseline design uses spontaneous synchrotron radiation produced in undulators as the source of hard x-rays.

Several undulator designs are under consideration, covering a large trade space that considers different magnet technologies and experimental needs, with parameters that are strongly coupled. For example the strength of the undulator magnetic field is constrained by the magnet technology; higher energy photons require a smaller period and/or lower undulator magnetic field; the photon flux decreases with decreasing undulator magnetic field.

Undulator magnetic fields of less than $1 \mathrm{~T}$ are required to radiate photons with energies above $2 \mathrm{keV}$ from $2.5 \mathrm{GeV}$ electrons. Such magnetic fields can be achieved using permanent magnet undulators of proven design. Alternatively, superconducting undulators may achieve the design specifications and may provide simplified operations in adjusting polarization of the photon beam, for example in a helical device.

To achieve the required large photon flux per bunch, undulators with length of up to $6 \mathrm{~m}$ are under consideration. These have particular advantage in reducing the charge per bunch 
requirement. Manufacturing complexity may require that these undulators be segmented. A final design will be motivated by the need for performance and avoidance of unnecessary design/engineering, manufacturing, and reliability risks.

\section{Synchronization}

Several synchronization concepts have been considered. For the baseline design, the deflecting cavities are driven from a highly stable rf signal derived from a mode-locked laser oscillator that is also the origin of the pump laser pulses. Using feedback on all $\mathrm{rf}$ systems (rf gun, injector linac, main linac, deflecting cavities) control of the bunch arrival time with respect to the rf phase at the deflecting cavities is expected to be better than 1 ps. Each cavity in the linacs is individually powered and controlled with phase and amplitude feedback systems to provide optimal stability.

The recirculating geometry allows the use of timing signals generated by the electron beam in a prior pass to be used as triggers for lasers and detection systems in succeeding passes through the machine. Optical undulators may be used to provide these timing signals, as may broadband synchrotron radiation from bend magnets.

The exploration of the limits of synchronization of x-ray pulses from the recirculating linacbased light source will continue. Analysis to-date suggests that the baseline design will provide the required synchronization between electron bunches, x-ray pulses, and the pump lasers.

\section{REFERENCES}

[1]. Schoenlein, R.W., et al., " Generation of femtosecond pulses of synchrotron radiation", Science, 2000. 287: p. 2237-2240

[2] Daresbury 4GLS: http://www.4gls.ac.uk/,

[3] Cornell energy recovery linac: http://erl.chess.cornell.edu/,

[4] BNL photoinjected energy recovery linac: http://nslsweb.nsls.bnl.gov/nsls/org/PERL/ ]

[5] D.A. Kairan, G.N. Kulipanov, A.N. Skrinsky, and N.A. Vinokurov, "MARS-A project of the Difraction Limited Fourth Generation X-Ray Sourc.," Proc. 1998 Asian Part. Conf.

[6] BESSY FEL: http://www.bessy.de/lab profile/01.FEL/index.php?language=en,

[7] TESLA FEL: http://tesla.desy.de/new_pages/TDR_CD/PartV/fel.html

[8] SLAC LCLS: http://www-ssrl.slac.stanford.edu/lcls/ ]

[9] A. Zholents et al "Generation of subpicosecond x-ray pulses using rf orbit deflection," NIM A 425 (1999) 385-389.

[10] TESLA Technical Design Report, DESY 2001-011, March 2001.

[11] J.-P. Carneiro, H. T. Edwards, M. J. Fitch, W. H. Hartubg, M. Ferrario, "Emittance measurements at the A0 Photo-injector," Proc. XX International Linac Conference, Monterey, USA, 2000.

[12] D. Edwards et al, "The Flat Beam Experiment at the FNAL Photoinjector," Proc. XXth International Linac Conference, Monterey, 2000. 


\section{ACCELERATOR PHYSICS}

\section{General CONSIDERATIONS}

The lattice of the recirculating accelerator has been designed to meet all requirements for the femtosecond x-ray source. The design permits tunability of the time-of-flight parameters, betatron phase advances, and chromaticity over the different sections of the lattice. The photon production section has been designed with six identical cells with variable beta-functions at the undulator and bend magnet $\mathrm{x}$-ray source points. Tracking studies indicate that lattice design is insensitive to errors in magnetic field quality, but needs beam based alignment to correct for alignment errors.

Minimizing the footprint of the accelerator imposes a major constrain on global machine parameters such as a shape and a circumference of the outer ring, length of a photon production section, and the length of the main linac. The circumferences of the three inner rings are less constrained, however, although they must be carefully selected to allow the machine to be used in the energy recovery mode and with a high electron beam current provided by operation with multiple bunches within the accelerator at any given time. In the case of multi-bunch operation to provide a high average flux, a uniform spacing of all electron bunches in the linac including accelerating and decelerating bunches is highly desirable. The set of ring circumferences given in Table 3-1 provides a wide variety of choices for a bunch repetition rate out of the gun, leading to almost uniform bunch spacing in the $1.3 \mathrm{GHz}$ main linac. An extra half wavelength in Ring 4 is needed in order to launch the returning electron beam into the decelerating phase of the rf field in the linac.

All four rings of the machine posses mirror symmetry relative to a central line dividing the machine into two halves, simplifying the machine lattice and operational flexibility with the possibility of acceleration and deceleration of electrons in the same linac. Although we do not plan deceleration and energy recovery at this point, the possibility will be available if needed in the future.

The lattice design allows the option to increase the electron beam energy in the photon production section from $2.5 \mathrm{GeV}$ to $3.1 \mathrm{GeV}$, by increasing the energy gain in the main linac. This requires that the electron beam energy at the exit of the injector linac, and the magnetic strengths of all magnetic elements, will be simultaneously raised by the same factor.

Table 3-1 Ring circumferences

\begin{tabular}{|c|c|c|}
\hline Ring number & $\begin{array}{c}\text { Circumference, } \\
\mathrm{m}\end{array}$ & $\begin{array}{c}\text { Number of RF } \\
\text { wavelengths }\end{array}$ \\
\hline 1 & 186.793762 & 810 \\
\hline 2 & 205.473139 & 891 \\
\hline 3 & 236.605432 & 1026 \\
\hline 4 & 295.75679 & 1282.5 \\
\hline
\end{tabular}




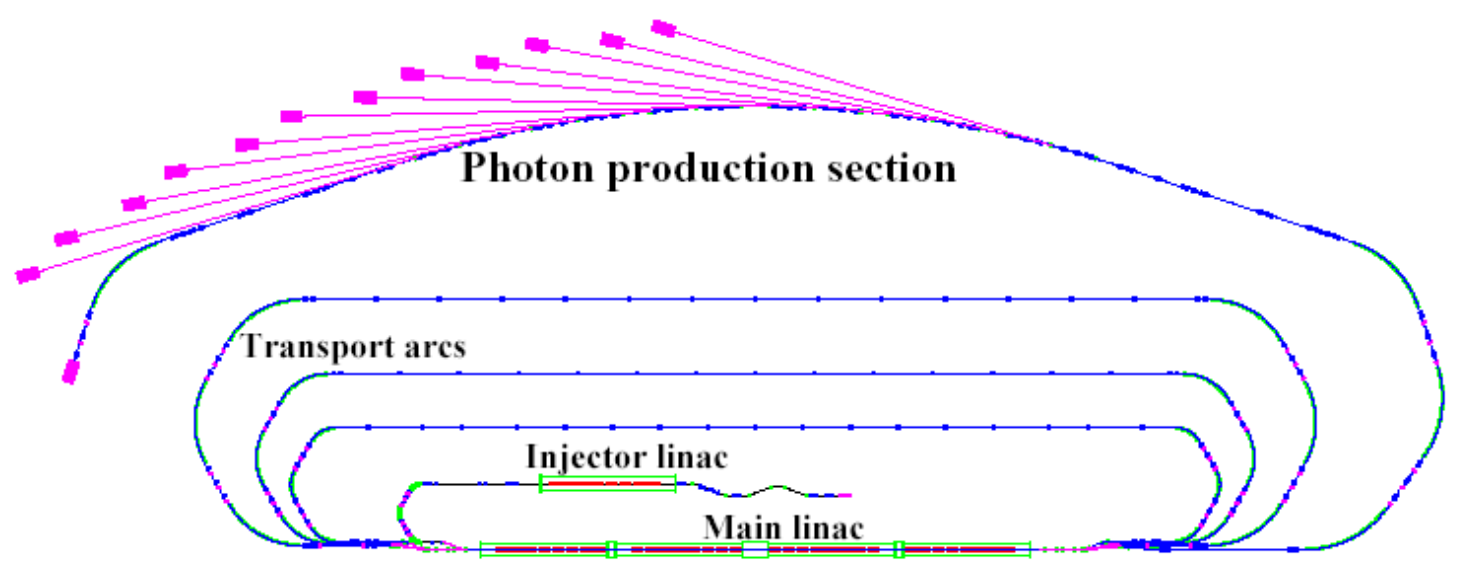

Figure 3-1 Machine layout, showing injector linac, main linac, four rings, and photon production section. Undulator and bend magnet beamlines are shown.

\section{LATTICE DESCRIPTION}

Here we describe the accelerator lattice in different sections of the machine, a shorter version of this description is given in the reference [1]. With the exception of a few specialized magnets in the beam combiner or beam spreader sections which transport the beams entering or exiting the main linac, all magnets are of a conventional electromagnet design similar to those of existing synchrotron radiation sources.

\section{Main Linac}

The main linac is the simplest part of the machine from a lattice point of view, having no dedicated beam focussing elements. Weak rf focusing is only visible at electron beam energies below few hundred $\mathrm{MeV}$. In all rings after the first the linac acts essentially as a drift space in transverse dynamics - the beta-functions expand quadratically towards both ends of the linac. This behavior is shown in Figure 3-2 for the first ring where beta-functions are modified by the rf focusing, and in Figure 3-3 for the linac section of the fourth ring, where a simple free drift is observed.

\section{Beam Spreader and Combiner}

A beam spreader section (see, Figure 3-4) transports the electron beam at various energies from the linac into the respective higher-energy rings. Its mirror image a beam combiner reconciles different energy orbits from the respective rings into a single line in the main linac. The inevitable proximity of beamlines of different energies results in these sections being rather compact and sophisticated parts of the lattice.

The magnet $\mathrm{B} 0$ (with the field of $1.14 \mathrm{~T}$ and the length of $0.82 \mathrm{~m}$ ) begins the orbit separation. This is a rectangular magnet with the entrance and exit faces orthogonal to a line parallel to the main linac, through which all energy beams pass. The bend angles for beams at different energies are $22.276^{\circ}$ for $720 \mathrm{MeV}, 11.933^{\circ}$ for $1.32 \mathrm{GeV}, 8.172^{\circ}$ for $1.92 \mathrm{GeV}$, and $6.218^{\circ}$ for $2.52 \mathrm{GeV}$. 


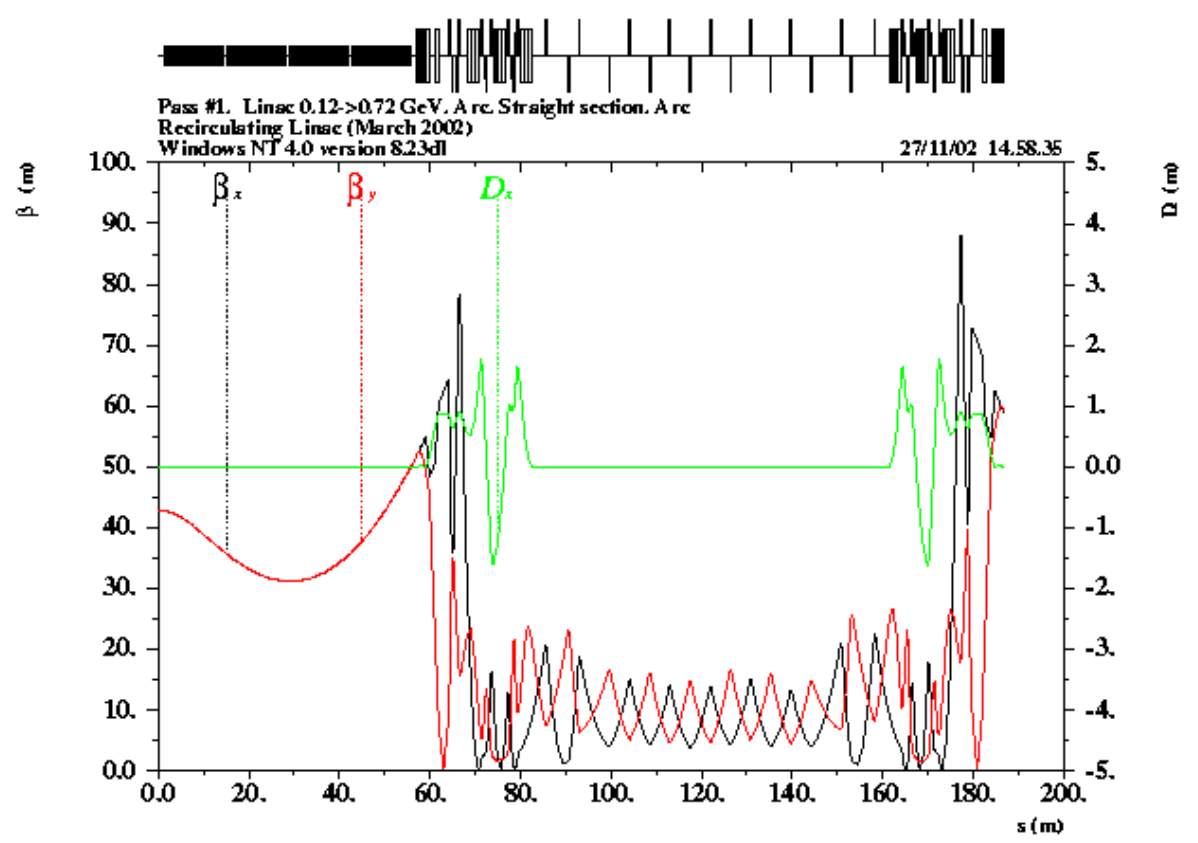

Figure 3-2 The lattice functions in Ring 1 (first pass through the main linac). The lattice of all other rings is similar with the exception of the beginning of the linac where effects of the rf focusing are visible in this ring only.

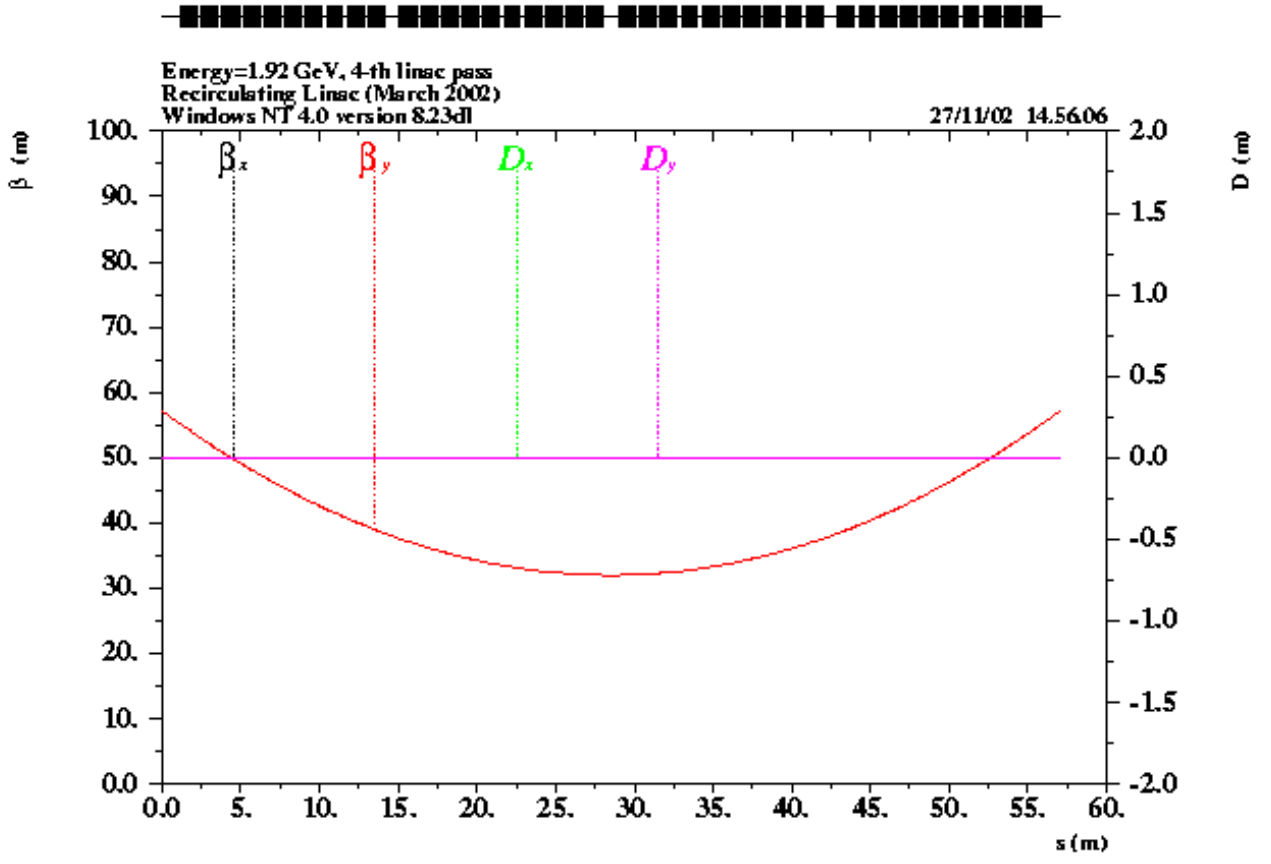

Figure 3-3 Lattice functions in the main linac for the fourth pass. 


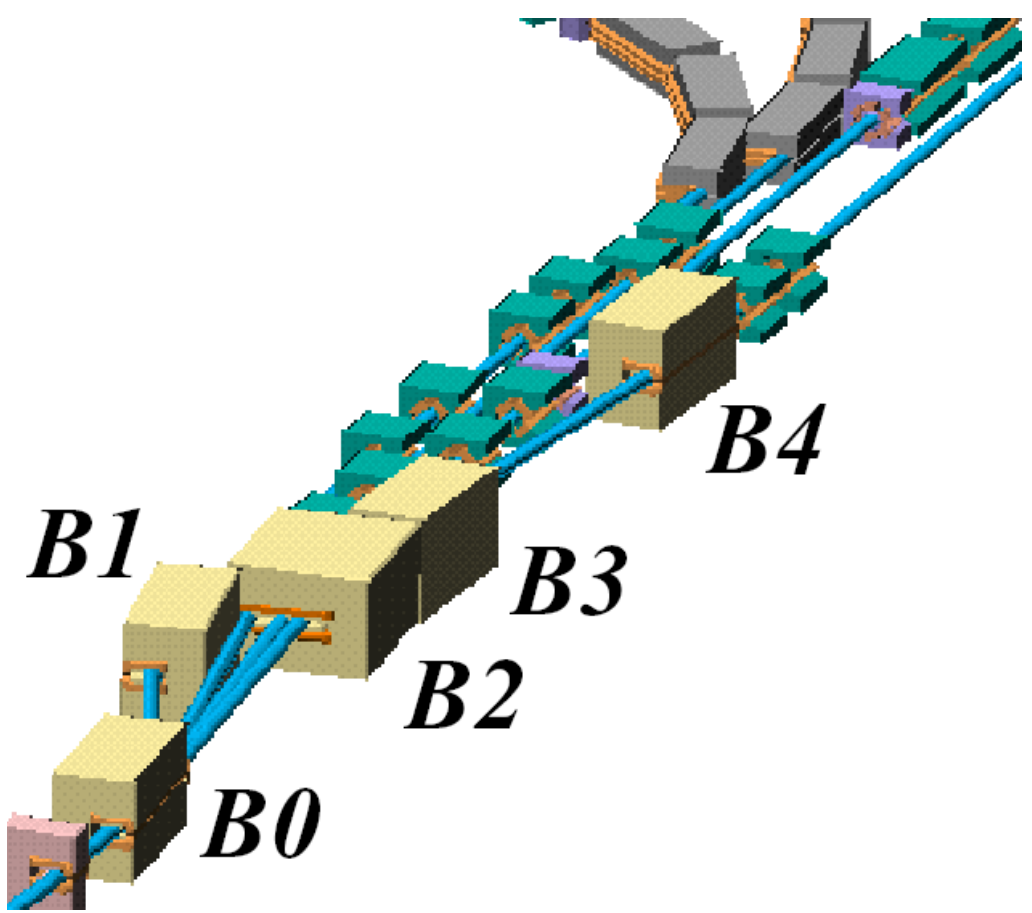

Figure 3-4 The beam spreader section, where the electron beam at different energies is directed into arcs following each pass through the linac.

Downstream magnets B1 and B2 are also rectangular magnets with the same length and oriented similarly to B0, and have equal strength but opposite polarity magnetic field. These magnets complete the separation of different energy beams into the rings 1, 2, and 3 and constrain a dispersion Courant-Snyder invariant excited in B0.

After B1 and B2 all orbits are parallel with the following separations between them: $175.7 \mathrm{~mm}$ between the $720 \mathrm{MeV}$ beam and $1.32 \mathrm{GeV}$ beam, $188.4 \mathrm{~mm}$ between the $1.32 \mathrm{GeV}$ beam and the $1.92 \mathrm{GeV}$ beam, and $96.7 \mathrm{~mm}$ between the $1.92 \mathrm{GeV}$ beam and $2.52 \mathrm{GeV}$ beam. The separation of the last two orbits is sufficient to allow room for a septum magnet B3 (see, Figure 3-5a) with the same polarity as B2. This magnet affects only the $2.52 \mathrm{GeV}$ beam and turns it away from the orbit of $1.92 \mathrm{GeV}$ beam. Finally the magnet $\mathrm{B} 4$ completes the separation of $2.52 \mathrm{GeV}$ beam orbit. The four magnets B0, B2, B3 and B4 form a chicane with zero orbit displacement and zero dispersion at the end.

In addition to dipole bending magnets, there are four quadrupole lenses on each of the three lower energy beamlines and two quadrupoles on the $2.52 \mathrm{GeV}$ beamline. These magnets are used for matching the beta-functions in the linac to the beta-function of each of the respective arcs. Because of the compact geometrical arrangement of beamlines in this region, the quadrupole locations are carefully chosen so that they do not result in spatial conflicts. The mechanical design of these quadrupoles shown in Figure 3-5b is also compact in the horizontal plane.

Two sextupoles on each of the spreader/combiner beamlines are used to control chromaticity and the $\mathrm{T}_{566}$ time-off-flight parameter to produce isochronous transport lines. 

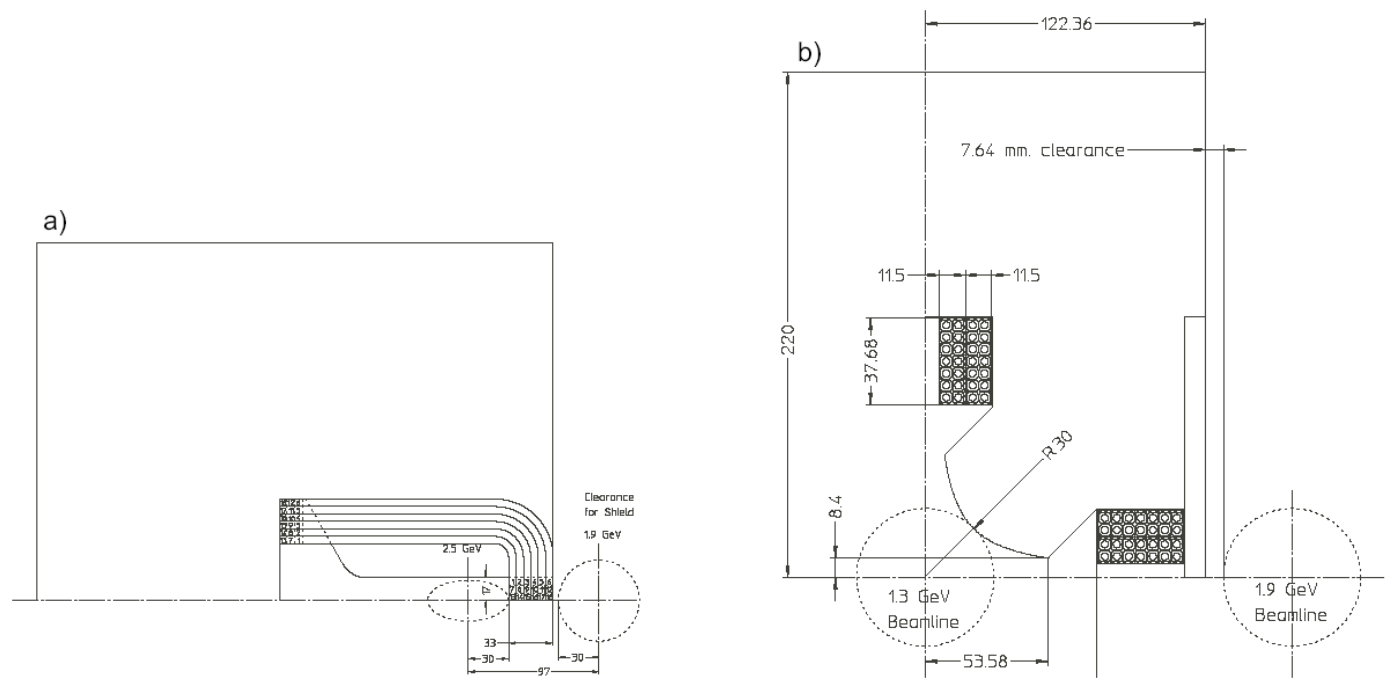

Figure 3-5 Crossections of a beam spreader septum bend magnet B3 (a) and a compact quadrupole (b).

\section{Magnetic arcs}

The arc sections transport electrons of a given energy from the end of the beam spreader to the corresponding straight section for each ring (or photon production section in the case of the Arc 4), and back to the beginning of the beam combiner.

The magnetic arcs each have a similar lattice, and are comprised of three $\sim 120^{\circ}$ betatron phase advance cells each containing a string of bending magnets, three quadrupoles and three sextupoles. The third cell of each arc is used for matching of the arc optical functions to those of the adjoining straight sections.

The electron beam transport from the end of the linac to the beginning of straight sections is achromatic and isochronous. However, changes in the time-of-flight parameter $\left(\mathrm{R}_{56}\right.$ matrix coefficient) can easily be provided should electron bunch length adjustment be required at various stages of acceleration (and possibly deceleration).

Sextupoles are used to compensate chromaticity and second order terms affecting isochronicity.

The dipole bending magnets, quadrupoles and sextupoles in each of the arcs are mechanically identical, allowing cost savings and simplicity.

\section{Straight sections}

All straight sections have a simple FODO lattice containing two identical betatron function matching sections at the two ends and a tune trombone in the center. The tune trombone consists of four cells, allowing the variation of the betatron tunes over a wide range of approximately \pm 0.5 with just two parameters. The center section in Figure 3-2 shows typical beta-functions in the straight section. 


\section{Photon production section}

Figure 3-6 shows beta and dispersion functions of the final pass through the linac and the highest energy beamline, Ring 4. The central part of this plot is the photon production section, which has six triple bend achromat cells with an arc angle of $6^{\circ}$ per cell. The vertical and horizontal betatron phase advances per cell are $\pi$ and $1.8 \pi$. All cells begin and end in the middle of a short straight section, whose length is sufficient to host a 2-m undulator. The vertical and horizontal beta-functions in the center of the undulator are $2.5 \mathrm{~m}$ and $7.5 \mathrm{~m}$.

The magnetic field of the central bend of a cell is $2 \mathrm{~T}$, and it may also be used as a source of hard x-rays. The vertical and horizontal beta-functions in this bend are $1.73 \mathrm{~m}$ and $3.4 \mathrm{~m}$ and the dispersion function is $6 \mathrm{~cm}$.

3.9 GHz rf structures operating in the first dipole mode are located between the arc and the beginning of the photon production section. The vertical beta-function at the location of these cavities is raised to $90 \mathrm{~m}$ to provide an efficient time-dependent transverse kick needed for the following x-ray bunch compression. The transfer matrix between this rf structure and similar location at the other end of the photon production section is a unit transfer matrix in both planes, to allow possible future cancellation of the kick by another set of similar cavities.

Due to the relatively low electron beam energy the effect of a synchrotron radiation on the electron beam is practically negligible. Even at $2.5 \mathrm{GeV}$ the average total energy loss of electrons in the undulators is approximately $500 \mathrm{keV}$, and the increase of the energy spread due to quantum fluctuations is approximately $50 \mathrm{keV}$ (well below the beam energy spread of $200 \mathrm{keV}$ of the incoming beam).

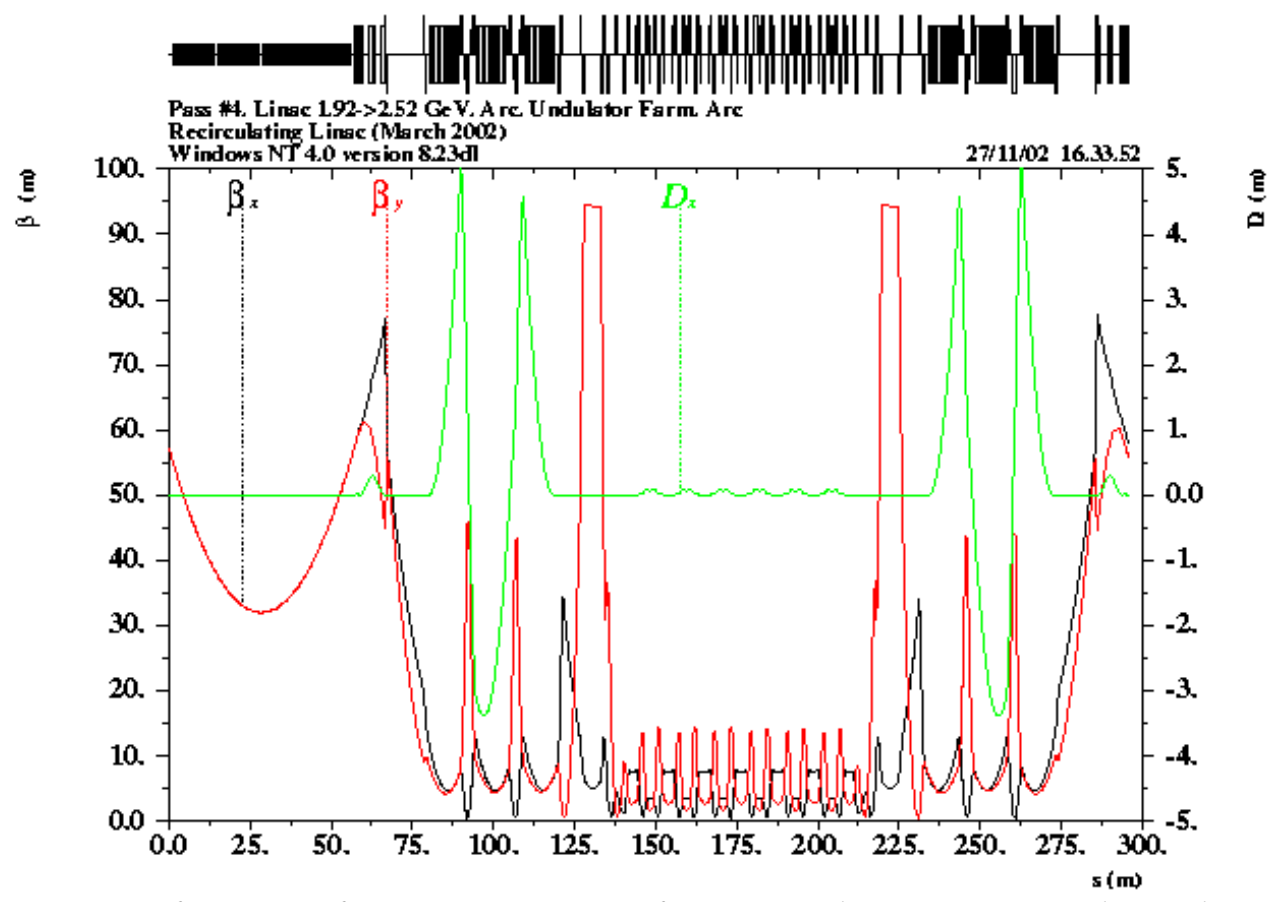

Figure 3-6 Lattice functions for Ring 4 starting from main linac entrance through to the photon production section and a returning arc and beam combiner to the linac. In the baseline design, the returning arc will not be built. 


\section{Tracking studies}

Single particle dynamics in the recirculating linac has been studied using the code COSY INFINITY [2]. This code uses a Taylor series map that allows fast tracking and readily provides parameter dependence studies between different quantities of interest. At the same time the deviation from symplecticity caused by truncation in the Taylor series is not important in a single pass system like a recirculating linac.

A typical result for the path length difference for electrons with a $0.1 \%$ rms energy spread propagating in a single arc is shown in Figure 3-7 for Arc3 (the arc of the third ring). Figure 3-7 shows histograms of time-of-flight through the arc for cases without sextupole correction applied (red) and with sextupole correction applied (green). Although the FWHM of the histogram before sextupole correction is small ( $\sim 10 \mathrm{fs})$, the tail extends to $\sim 200 \mathrm{fs}$, which is undesirable. This is caused by second order time-of-flight terms in the lattice. Application of sextupoles can be seen to effectively reduce these terms such that the tails are confined within $50 \mathrm{fs}$. The impact of the sextupoles on beta-functions, betatron phase advances and off-momentum orbit is not significant.

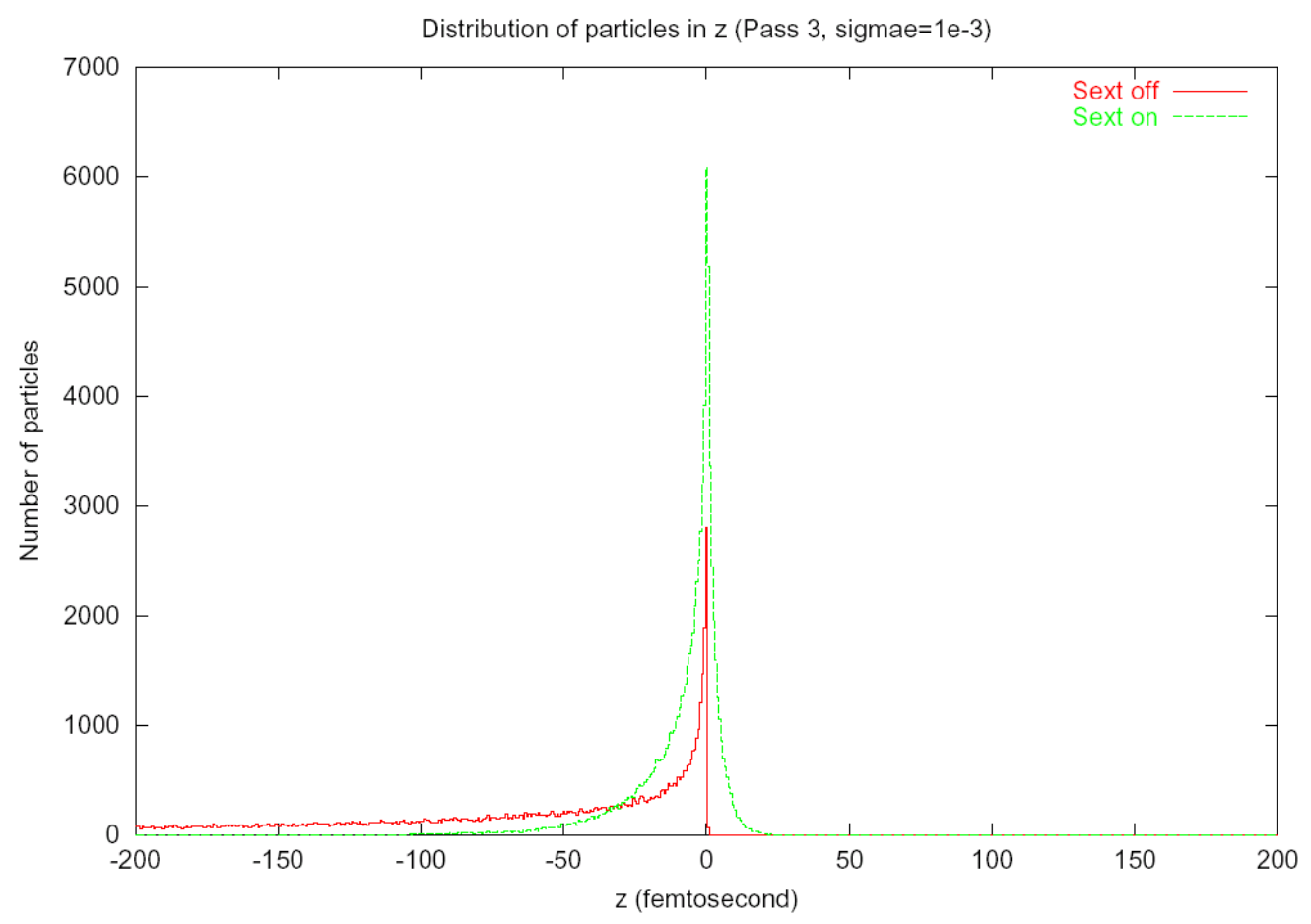

Figure 3-7 Histograms of pathlengths in Arc 3 (the arc of Ring 3) without sextupole correction (red) and with sextupole correction (green).

The effects of static random lattice errors given in Table 3-2 have been studied, without the effects of BPM errors. Table 3-2 also specifies beam parameters used in the tracking studies. In 
this study we found that alignment errors may cause vertical orbit distortions of the order of few millimeters for an uncorrected orbit. In this case the off-center beam in the sextupoles induces a large coupling between horizontal and vertical planes. This is seen for few seeds in Figure 3-8, which shows the vertical beam profile at the end of Arc3. The effect is readily corrected by use of steering magnets to reduce the beam offset to $\sim 200 \mu \mathrm{m}$, resulting in a vertical beam size a few percent different from the nominal. Figure 3-9 shows the vertical beam profile at the end of Arc3 for the case of orbit correction applied. The exception was found in one seed, where in addition to orbit correction we needed to re-optimize the strength of the sextupoles in order to return to the nominal beam size.

Table 3-2 Beam parameters and errors, all quantities are rms values.

\begin{tabular}{|l|r|}
\hline & \multicolumn{1}{|c|}{ Beam Parameters } \\
\hline Horiz. emittance (normalized, $\pi$ mm mrad) & 20 \\
Vert. emittance (normalized, $\pi$ mm mrad) & 0.4 \\
Momentum spread & $1 \times 10^{-3}$ \\
\hline & \multicolumn{1}{|c|}{ Errors* } \\
\hline Tilt $(\mathrm{mrad})$ & 0.2 \\
Misalignment in x $(\mu \mathrm{m})$ & 150 \\
Misalignment in y $(\mu \mathrm{m})$ & 150 \\
Misalignment in z $(\mathrm{mm})$ & \\
Setupole field in bend magnets & $\mathrm{b} 3 / \mathrm{b} 1=10^{-4}$ at $\mathrm{r}=3 \mathrm{~cm}$ \\
Sextupole field in quadrupoles & $\mathrm{b} 3 / \mathrm{b} 2=10^{-4}$ at $\mathrm{r}=5 \mathrm{~cm}$ \\
Setting errors for all magnets & $\Delta \mathrm{B} / \mathrm{B}=1 \mathrm{e}-3$ \\
\hline
\end{tabular}

* For all errors we assume gaussian distribution truncated at $2.5 \sigma$.

To allow global tracking of the entire machine a simulation code was developed based on symplectic integration. This code is designed using a concept of fiber bundles such that the fundamental component of a beam line is the discrete variable $s$ on which physical data is attached as well as the geometrical transformations connecting the description of an individual magnet to the external measurable parameters at that particular $s$. This allows modeling the recirculation of particles with different energies through common elements, and having them described in the lattice just once.

\section{INJECTION SYSTEM}

In this section we consider the part of the injection system from the end of the photocathode gun to the entrance of the main linac. In the present analysis we are mainly concerned with the preservation of the longitudinal phase space during the acceleration in the injector linac of the 20 ps long electron bunch exiting the rf gun, followed by its compression to 2 ps in the arc connecting the injector to the main linac. We prefer not to produce a bunch of length less than 2 ps because of peak-current dependent collective effects, in particular coherent synchrotron radiation (CSR), (see Chapter 4-Collective Effects). At the same time a 2 ps long electron bunch 


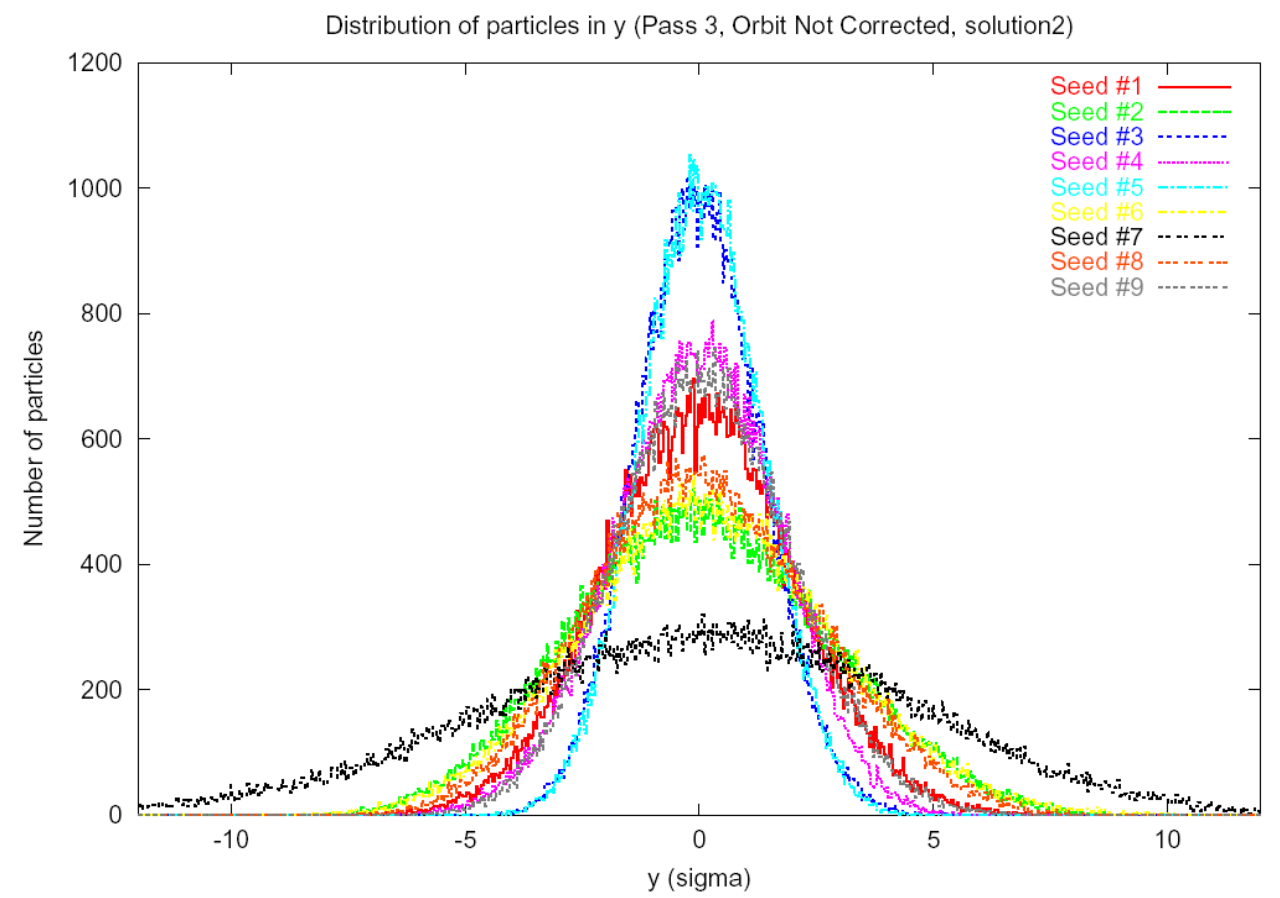

Figure 3-8 Vertical beam profile at the end of Arc3 for a range of error seeds, no orbit correction.

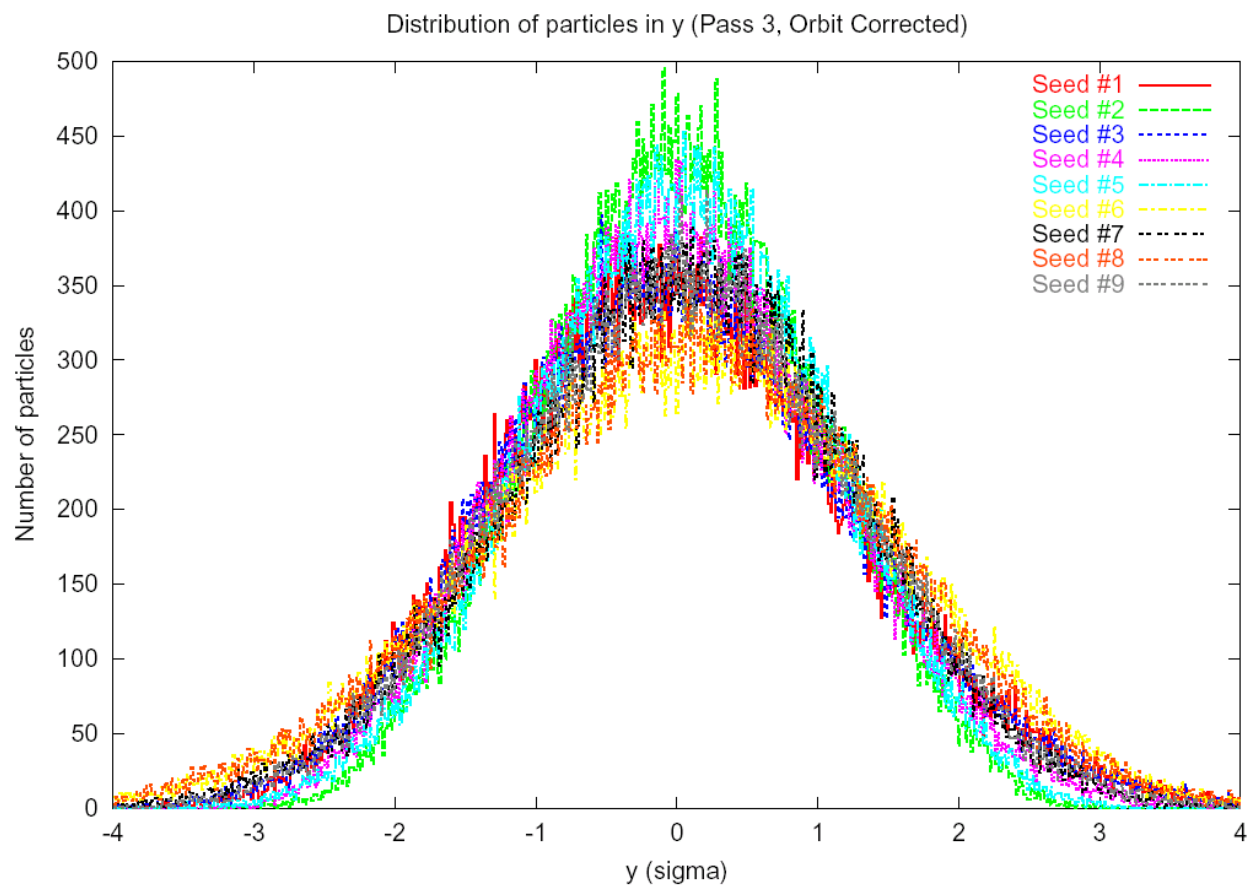

Figure 3-9 Vertical beam profile at the end of Arc3 for the same range of error seeds, with orbit correction applied.

is short enough that a cosine-like energy variation along the bunch resulting from the acceleration on the crest of the rf field in the main linac is small and does not degrade generation of femtosecond $\mathrm{x}$-ray pulses. 
Figure 3-10 shows a schematic of the injection system. The injector linac consists of seven $1.3 \mathrm{GHz}$ TESLA superconducting rf cavities combined in one cryomodule. The electron beam supplied by the photocathode gun gains approximately $110 \mathrm{MeV}$ in this linac. The beam is then injected into a smaller $\sim 13 \mathrm{MeV}$ linac operated at $3.9 \mathrm{GHz}$ (third harmonic of $1.3 \mathrm{GHz}$ ). The second linac acts to linearize the longitudinal phase space distortions introduced by the rf gun and injector linac [1], i.e. it produces an output beam with linear head-to-tail energy variation along the bunch which allows for subsequent bunch compression.

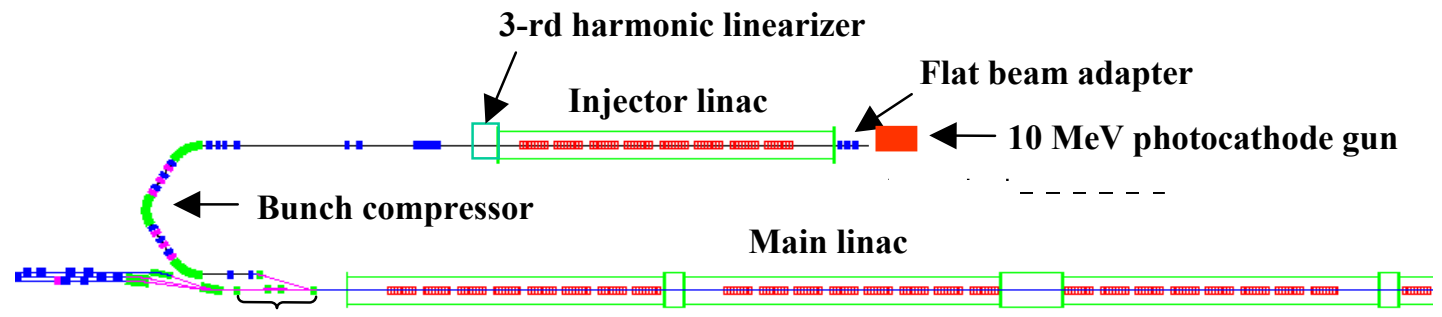

Injection chicane

Figure 3-10 Schematic of the injector system

We use the following considerations to define the required amplitude of the accelerating field $u_{3}$ and equilibrium $\mathrm{rf}$ phase $\varphi_{3}$ in the linearizer. First we write the total field acting on the electron at a position $s$ relative to the bunch center:

$$
U(s)=u_{1} \operatorname{Cos}\left(k_{R F} s\right)+u_{3} \operatorname{Cos}\left(\varphi_{3}+3 k_{R F} s\right)+a\left(k_{R F} s\right)+b\left(k_{R F} s\right)^{2}
$$

Here we assume zero equilibrium phase in the injector linac and define $k_{R F}$ and $u_{1}$ as the wave vector and amplitude of the accelerating field in the injector linac. We show in the Chapter 4Collective Effects that in the case of the uniform rectangular charge density distribution the longitudinal wake is a quadratic polynomial with respect to $s$. Therefore we include it in (1) using coefficients $a$ and $b$ that can be easily obtained from Equation 24 of Chapter 4-Collective Effects. The field acting on the electron at the equilibrium phase is:

$$
U(0)=u_{1}+u_{3} \operatorname{Cos}\left(\varphi_{3}\right)
$$

Thus the difference $\Delta U(s)=U(s)-U(0)$ shows the variation of the electron energy along the bunch. For a relatively small $k_{R F} s$ we can produce a linear energy variation by requiring a zero sum of all quadratic terms $\left(k_{R F} s\right)^{2}$ in $\Delta U$. This gives a condition:

$$
u_{3} \operatorname{Cos}\left(\varphi_{3}\right)=\frac{u_{1}-2 b}{9}
$$


If the bunch length is to be compressed by a factor $M$ and the initial energy spread of electrons is $\delta E$, than the linearizer should create an energy variation $M \delta E$ from head to tail of the electron bunch. This implies the following condition:

$$
u_{3} \operatorname{Sin}\left(\varphi_{3}\right)=-\left(\frac{M \delta E / e}{3 k_{R F} I_{b}}+\frac{a}{3}\right)
$$

where $l_{b}$ is the bunch length and $e$ is the electron charge. Thus $u_{3}$ and $\varphi_{3}$ are defined by Eqs. 3 and 4. For a set of parameters specific to our injector, we calculate $u_{3}=-12.6 \mathrm{MV}$ and $\varphi_{3}=-$ 4.9 ${ }^{\circ}$. Figure 3-11 illustrates the effect of the linearization, showing the evolution of the longitudinal phase from the beginning of the injector linac, where particles are launched on a window frame in phase space with dimensions $\pm 10 \mathrm{ps}$ and $\pm 20 \mathrm{keV}$, to the end of the linearizer.

The linearizer is followed by a section to match the betatron functions into the bunch compressor, and then by the bunch compressor itself. The bunch compression is achieved in the arc that transports the electron beam into the main linac. The electrons with approximate energy of $120 \mathrm{MeV}$ merge into the recirculating linac in the last magnet of the injection chicane (part of the beam combiner). The design of the bunch compressor allows variation of $R_{56}$ over a wide range from $0.75 \mathrm{~m}$ to $1.9 \mathrm{~m}$. Compressing a $20 \mathrm{ps}$ long bunch into a $2 \mathrm{ps}$ bunch requires:
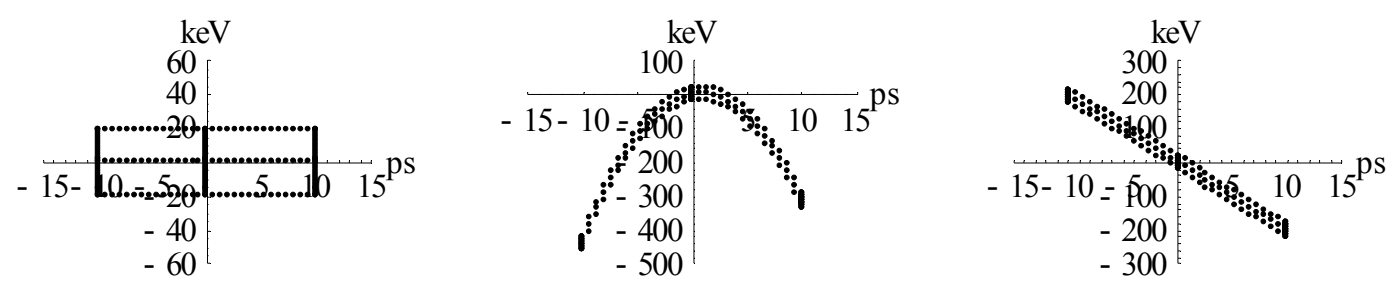

Figure 3-11 The evolution of the longitudinal phase space from the beginning of the injector linac (left), after the linac and before the linearizer (center), and after the linearizer (right).

$$
\mathrm{R}_{56}=\frac{l_{b}}{M} \frac{\left(u_{1}+u_{3}\right)}{\delta E / e}=1.61 \mathrm{~m}
$$

Figure 3-12 shows the lattice optical functions in the bunch compressor. The compressor beamline consists of two and a half cells each containing a bend magnet, three quadrupoles, and three sextupoles. Sextupoles are used to control chromaticity and the second order time-of-flight parameter $T_{566}$. Three phase-space plots of a compressed electron bunch are shown in Figure 313. The left plot shows the case when no CSR is included, the central plot is for a case with CSR, and the right plot shows the phase space at the end of the first pass of the main linac. The electrons were accelerated at $7^{\circ}$ off the crest in order to partially correct the correlated head-totail energy variation created by CSR. 


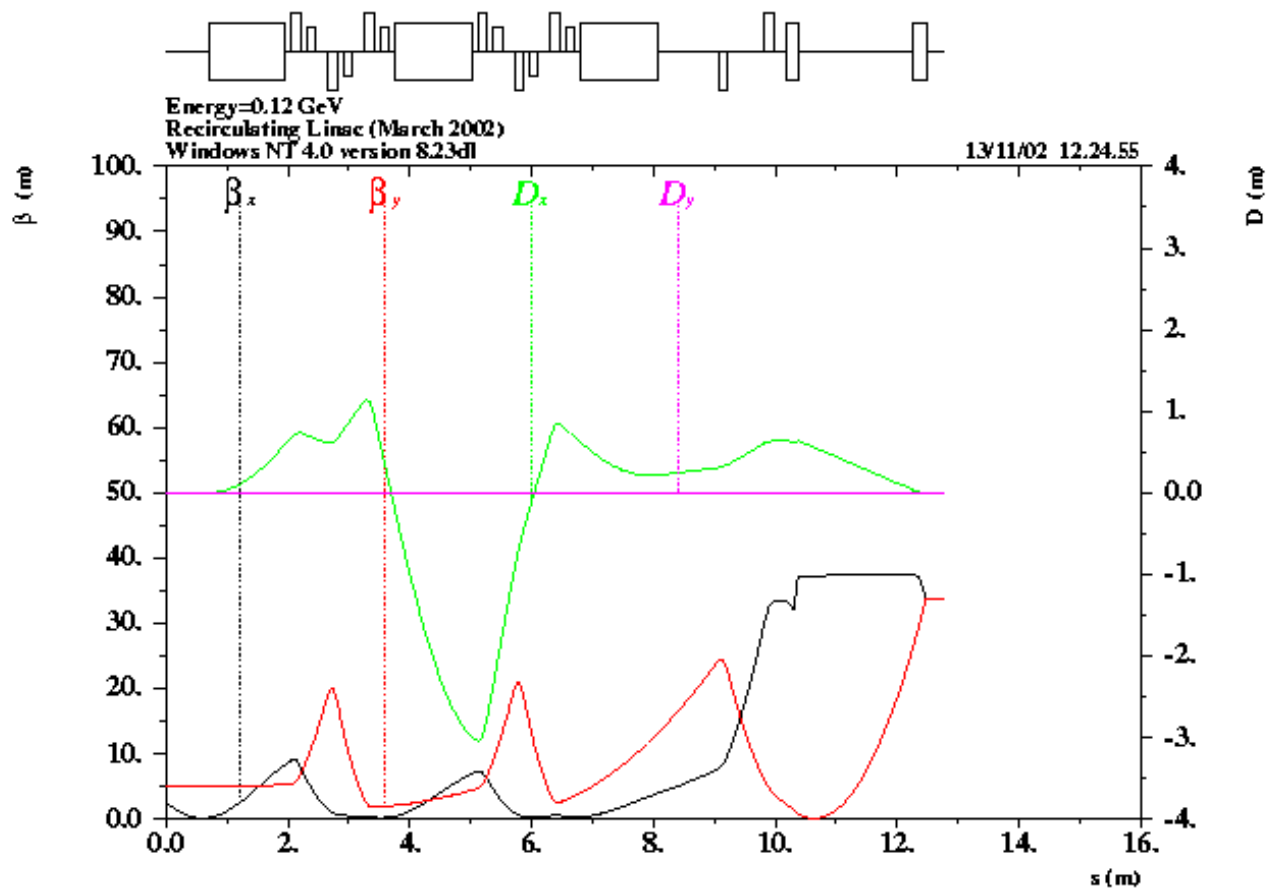

Figure 3-12 Lattice optical functions in the bunch compressor, for $R_{56}=1.61$.

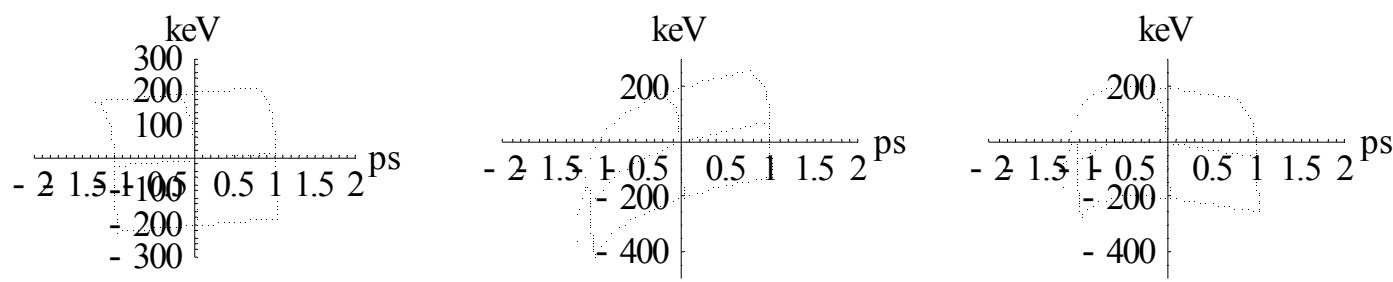

Figure 3-13 Longitudinal phase space for a compressed electron bunch: without CSR (left plot), with CSR (central plot), and after the main linac and partial compensation of CSR (right plot).

We now consider effects of timing jitter. Figure 3-14 shows the evolution of the longitudinal phase space starting with an initial time shift of 3 ps for a special case where we exclude the longitudinal wake. As seen here, the temporal offset becomes an energy offset following the bunch compressor. This results from the $90^{\circ}$ rotation in the phase space introduced by the compressor, and works to our advantage since the rest of the machine is designed to be isochronous. 

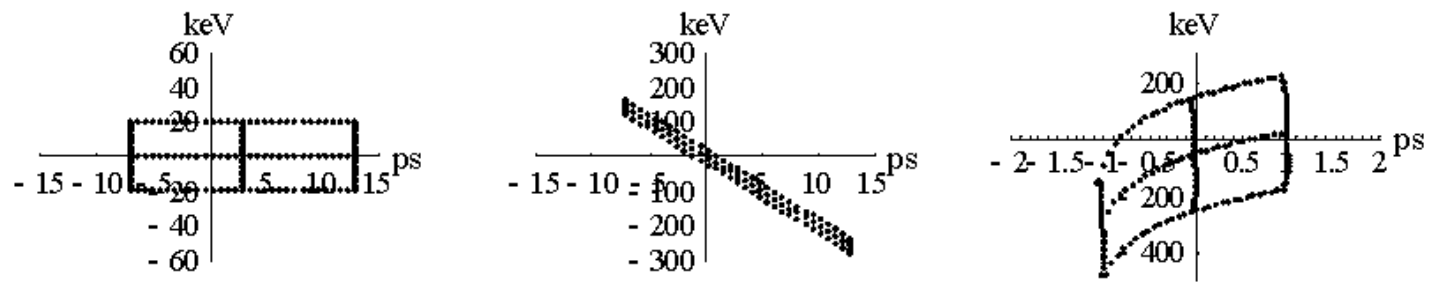

Figure 3-14 The evolution of the longitudinal phase space for a time delay of $3 \mathrm{ps}$ in the input beam (excluding the longitudinal wake): initial distribution (left), after the linearizer (central), and at the end of the bunch compressor (right).

The result becomes different with the inclusion of the longitudinal wake. As opposed to the linac rf field, the longitudinal wake is always synchronous with the electron bunch, and the linearization scheme shown above does not work perfectly for this case. Figure 3-15 shows the evolution of longitudinal phase space with the longitudinal wake included. Some energy off-set appears after the linearization (the phase space distribution outlined with red dots on the left plot), and this results in a time off-set after the bunch compressor (red dots on the right plot).

Nevertheless, it is possible to restore the correction by using two third harmonic linearizers instead of one, in this example a main linearizer with $u_{3}=-13.4 \mathrm{MV}$ and $\varphi_{3}=-3.5^{\circ}$ and a complimentary one with $u_{3}=-0.85 \mathrm{MV}$ and $\varphi_{3}=-162^{\circ}$. The settings for the second linearizer correspond to the longitudinal wake defined in Chapter 4-Collective Effects. It is preferable for the second linearizer to be built from warm cavities and have a relatively wide bandwidth, to allow the drive signal to be derived from arrival time of the electron bunch.
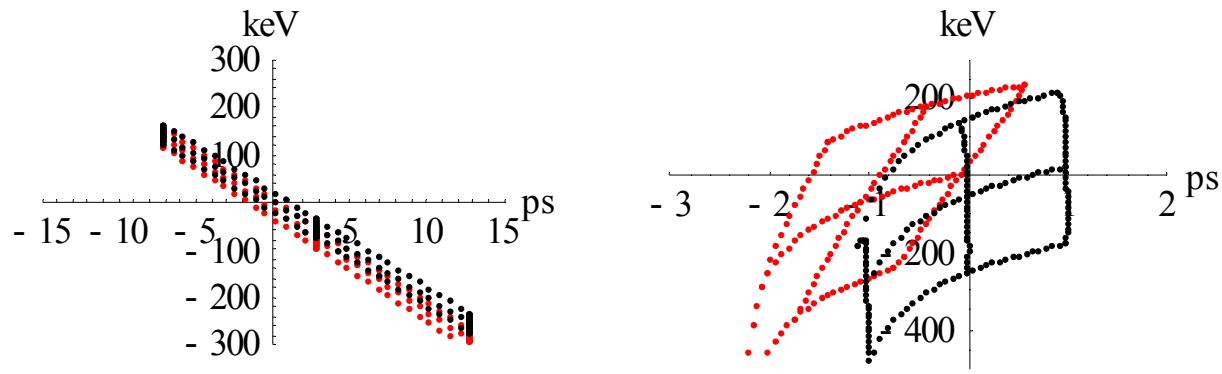

Figure 3-15 Evolution of longitudinal phase space with the longitudinal wake included.

\section{REFERENCES}

[1] A. Zholents et al., "Initial lattice studies for Berkeley femtosecond x-ray light source", proceedings of EPAC, Paris, 2002.

[2] M. Berz, NSCL Technical Report MSUCL-1088, Michigan State University, 1998.

[3] T.I. Smith, Proc. 1986 Linear Accelerator Conf., Stanford, CA, USA, p.421 


\section{COLLECTIVE EFFECTS}

\section{INTRODUCTION}

The proposed facility is designed to operate with a "flat bunch" requiring a small vertical emittance $\varepsilon_{\mathrm{y}} \approx 0.4 \mathrm{~mm}$-mrad, and large emittance ratio $\varepsilon_{\mathrm{x}} / \varepsilon_{\mathrm{y}} \approx 50$. Successful machine design depends critically upon emittance control from the election source at the rf photocathode to the radiation source point in undulators. The understanding and mitigation of collective effects is essential for emittance control. Key aspects of accelerator physics involved in beam break-up, coherent synchrotron radiation, resistive wall impedance and other effects have been examined and are reported here.

\section{BEAM BREAK-UP}

The Beam Break-Up (BBU) instability, generated by dipole modes in the linac excited by off-axis beam trajectories, can develop within a single bunch and lead to a dilution of the emittance [1]. As a bunch travels through an accelerating section, the head undergoes an unperturbed motion while the tail experiences a deflection due to the wakefield of preceding particles. Analogously, the same phenomenon can be observed when the structure is misaligned with respect to the ideal beamline, even if the bunch is injected on the nominal axis.

There are three aspects which require particular attention in the BBU analysis for a recirculating linac: the very wide energy range, $10 \mathrm{MeV}$ to $2.5 \mathrm{GeV}$, (including the injector linac); the recirculation through the main linac, which requires taking into account the longrange as well as the short-range wake; and the absence of focussing elements in the linac, which precludes the use of usual analysis methods based on the assumption that the beam energy does not change significantly in a betatron wavelength.

The equation describing the transverse displacement $x(s, z)$ of the electrons in an accelerated bunch, as a function of their longitudinal position within the bunch $z$, can be written in the form [2]:

$$
\frac{d}{d s}\left\lfloor\gamma(s) \frac{d x}{d s}\right\rfloor+k^{2}(s) \gamma(s) x(s, z)=r_{0} \int_{z}^{\infty} \rho\left(z^{\prime}\right) W_{\perp}\left(z^{\prime}-z\right) x\left(s, z^{\prime}\right) d z^{\prime}
$$

where $\gamma$ is the relativistic factor, $k$ the focussing strength, $r_{0}$ the classical electron radius, $\rho$ the bunch density, $W_{\perp}$ the transverse wake function per unit length and $s$ indicates the position along the linac. We assume infinitesimally small transverse beam dimensions (a reasonable approximation, when the bunch dimensions are much less than the size of the beam pipe), so that $x$ may be interpreted as the displacement of the center of a bunch slice. We also assume a bunch length much less than the betatron wavelength, therefore the displacement in the rhs of Equation (1) is not retarded and, finally, we use an average transverse wake function obtained from the wake of a single cavity [3], over the linac length. 
We first consider the effect associated with a displacement of the electron bunch at the injection into the perfectly aligned linac. We then extend the analysis to the case of misalignments of the linac rf cavities and cryomodules.

Throughout the report we compare the results obtained to the output of a simple tracking code, written as a Mathematica notebook (See BBU tracking code description in Appendix A of this Chapter).

In order to keep the analytical expressions reasonably simple, we model the linac length as entirely filled with rf cavities (thus neglecting all the drift spaces that account for as much as one fifth of the total length). This causes the wakefield intensity used in the analysis to be larger than the actual value and the average accelerating gradient to be smaller. It can be shown that this corresponds to a conservative estimate of the BBU growth. When comparing the analytical results to MERLIN output (c.f Figure 4-9) it must be kept in mind that the packing factor is only $80 \%$. Since the formulas we are going to derive next depend on the square for the linac length, we add a correction factor of $\sim 0.64$.

Since there are in focussing elements on the recirculated accelerating section, the solution of Equation (1) can be obtained using a perturbation technique. The first order solution $x_{(1)}$, with initial conditions: $x(s=0)=x_{0}, x^{\prime}(s=0)=x_{0}^{\prime}$ for all the electrons in the bunch, is [4]:

$$
x_{(1)}=x_{0}+x_{0} A_{0}\left\lfloor\frac{\Delta \gamma}{\gamma_{i}} \frac{s}{L}-\ln \left(1+\frac{\Delta \gamma}{\gamma_{i}} \frac{s}{L}\right)\right\rfloor+x_{0}^{\prime} L \frac{\gamma_{i}}{\Delta \gamma} \ln \left(1+\frac{\Delta \gamma}{\gamma_{i}} \frac{s}{L}\right)
$$

where

$$
A_{0}(z)=\frac{r_{0} \gamma_{i} L^{2}}{\Delta \gamma^{2}} \int_{z}^{\infty} \rho\left(z^{\prime}\right) W_{\perp}\left(z^{\prime}-z\right) d z^{\prime}
$$

and $L$ is the linac length, $\gamma_{i}$ the injection energy and $\Delta \gamma$ the total energy gain accumulated up to $s$ (i.e. taking into account eventual prior passes in the linac).

It is convenient to examine in detail the cases of coordinate and angular displacement at injection separately. For the numerical results the wake for the TESLA rf cavities [5] and a gaussian bunch $(\sigma=2 \mathrm{ps})$ are considered, but the formulas obtained below are valid for an arbitrary bunch distribution.

Coordinate Displacement and No Angular Displacement: $x(s=0, z)=x_{0}, x^{\prime}(s=$ $0, z)=0$.

If we assume no angle error at injection Equation (2) becomes at the end of the $n$-th linac pass

$(s=n L)$

$$
\frac{x_{(1)}}{x_{0}}=1+A_{0}\left\lfloor\frac{\Delta \gamma}{\gamma_{i}}-\ln \left(1+\frac{\Delta \gamma}{\gamma_{i}}\right)\right\rfloor
$$


Figure 4-1 shows the relative displacements, $x_{(1)} / x_{0}-1$, along the bunch, with respect to the bunch head, at the end of each pass in the linac, which is the source of vertical emittance growth.

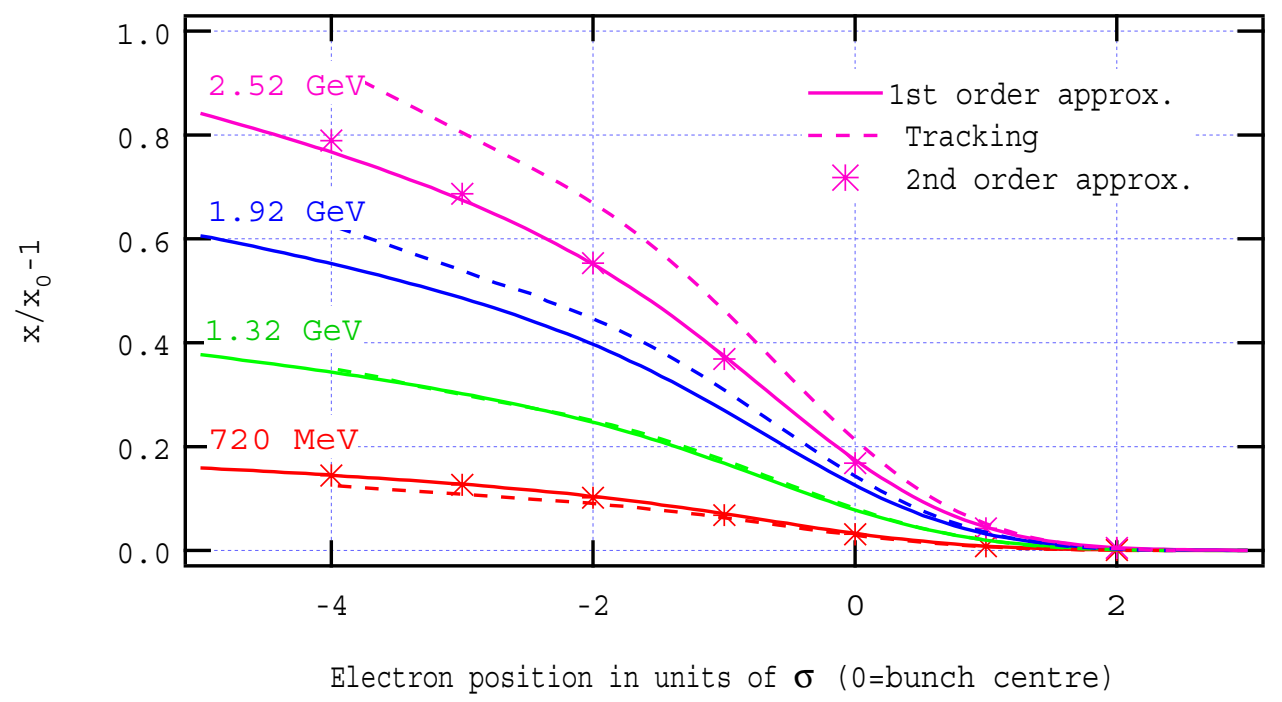

Figure 4-1 Cumulative transverse displacement (relative to the bunch head), as a function of the electron position within the bunch, after each pass, for coordinate displacement $x_{o}$ at the entrance to the linac.

After the first pass the bunch tail is displaced by almost $20 \%$ of its initial displacement at injection with respect to the bunch head. As this value is non negligible, we also obtained the second order solution of Equation (1), to verify that the iterative method converges rapidly and that the first order solution is already adequate. The values obtained, at several positions along the bunch, are shown by asterisks in Figure 4-1. We conclude that the first order approximation can be safely used. In this model the beam transports directly from the end of the linac back to the beginning, and so additional passes are equivalent to having a longer linac. The second order approximation at the highest energy begins to be somewhat different from the first order result.

The dashed curves in Figure 4-1 are the output from our tracking code. The agreement between the analytical formula and the tracking code becomes worse at higher energies, corresponding to a longer linac, where the effect of the approximation made in the analysis become appreciable.

Figure 4-2 shows the transverse displacement at the end of the four linac passes for two different bunch lengths $(1.5$ and $3 \mathrm{~mm}$ ) and two different charge distributions (gaussian and uniform). There are no significant differences in effects between the two distributions for a given bunch length, while the effect of the bunch length itself is substantial.

Angular Displacement and No Coordinate Displacement: $x(s=0, z)=0, x^{\prime}(s=$ $0, z)=x_{0}^{\prime}$.

In this case, the second order solution must be applied to improve our approximation: 


$$
\frac{x_{(2)}}{x_{0}^{\prime} n L}=\frac{\gamma_{i}}{\Delta \gamma} \ln \left(1+\frac{\Delta \gamma}{\gamma_{i}}\right)+A_{0} \frac{\gamma_{i}}{\Delta \gamma}\left\lfloor\left(2+\frac{\Delta \gamma}{\gamma_{i}}\right) \ln \left(1+\frac{\Delta \gamma}{\gamma_{i}}\right)-2 \frac{\Delta \gamma}{\gamma_{i}}\right\rfloor
$$

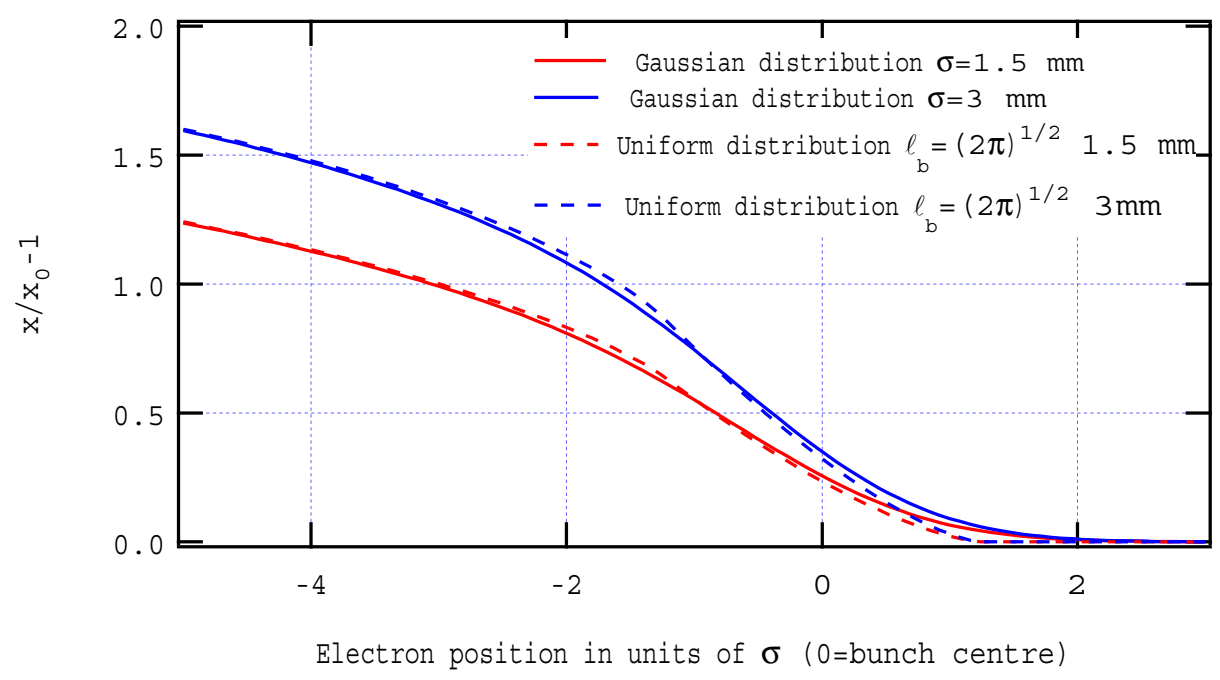

Figure 4-2 Displacement at the end of four passes for different bunch lengths and distributions, for coordinate displacement $x_{o}$ at the entrance to the linac.

Figure 4-3 shows numerical results for the baseline parameters. The vertical axis shows the transverse position of the center of charge of each transverse slice along the bunch, with respect to the bunch head.

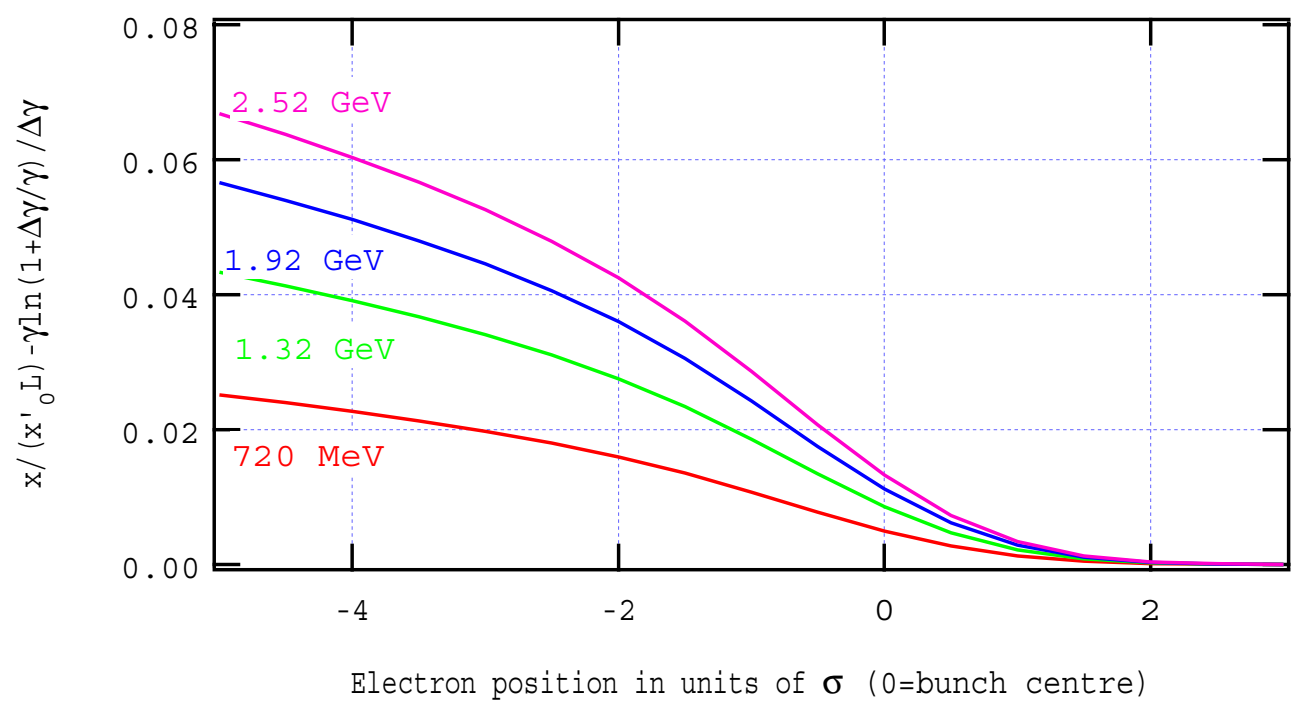

Figure 4-3 Electron displacement as a function of the position within the bunch (relative to the bunch head), for angular displacement $x_{o}^{\prime}$ at the entrance to the linac. 
Comparing Figures 4-1 and 4-3, we observe that the effect of an angle error seems less significant than that of an injection offset, and this difference becomes more pronounced at higher energies.

\section{Misalignment Effects}

In the previous section it was assumed that the accelerator structure is perfectly aligned and the wake field is produced as a consequence of beam injection with a displacement or angle error. In this section the effects of misalignments of the rf cavities and cryomodules on the transverse dynamics is presented.

To begin with, consider only errors in the cavity alignment. This appears as an extra term on the rhs of Equation (1):

$$
\frac{d}{d s}\left\lfloor\gamma(s) \frac{d x}{d s}\right\rfloor+k^{2}(s) \gamma(s) x(s, z)=r_{0} \int_{z}^{\infty} \rho\left(z^{\prime}\right) W_{\perp}\left(z^{\prime}-z\right)\left[x\left(s, z^{\prime}\right)+d_{c}(s)\right] d z^{\prime}
$$

where the function $d_{c}(s)$ describes the misalignment, referred to an ideally perfectly aligned linac. We can again find an iterative solution of Equation (6) which, to first order, yields:

$$
x_{(1)}(s, z)=x_{0}+A_{0}\left\{x_{0}\left\lfloor\frac{\Delta \gamma}{\gamma_{i}} \frac{s}{L}-\ln \left(1+\frac{\Delta \gamma}{\gamma_{i}} \frac{s}{L}\right)\right\rfloor+\sum_{i=0}^{N_{c a n}-1} d_{c i} F_{i}(s)\right\}+x_{0}^{\prime} \ln \left(1+\frac{\Delta \gamma}{\gamma_{i}} \frac{s}{L}\right)
$$

where the functions $F_{\mathrm{i}}(s)$ can be analytically calculated [4] and take into account the influence of the various cavity misalignments, depending on their respective position along the linac.

From Equation (7) we can see that the effect of cavity misalignments is to introduce an additional displacement that, in first approximation, does not depend on the injection coordinates $x_{0}$ and $x_{0}^{\prime}$. Knowing the rms cavity misalignment, the wakefield-induced rms displacement can be calculated. Choosing $x_{0}=x_{0}^{\prime}=0$, for example, we find:

$$
\left\langle x^{2}\right\rangle^{1 / 2}=A_{0}(z) \sqrt{\sum_{i=0}^{N_{c a v}-1} F_{i}(s)}\left\langle d_{c}^{2}\right\rangle^{1 / 2}
$$

In calculating Equation (8), we have assumed no correlation between the alignment errors and this is strictly valid only when analyzing a single pass.

We can also account for the misalignments between the cryomodules in the same way finding equations analogous to Equations (6-8), but with the number of cryomodules $\mathrm{N}_{\text {mod }}$ and their length in place of the rf cavity number and length. Taking into account both alignment errors, Equation(8) becomes

$$
\left\langle x^{2}\right\rangle^{1 / 2}=A_{0}(z) \sqrt{\sum_{i=0}^{N_{c a v}-1} F_{i}(s)\left\langle d_{c}{ }^{2}\right\rangle+\sum_{i=0}^{N_{\text {mod }}-1} G_{i}(s)\left\langle d_{m}{ }^{2}\right\rangle}
$$


where the functions $G_{\mathrm{i}}(s)$ are analogous to $F_{\mathrm{i}}(s)$, but for cryomodules rather than for individual cavities.

We obtain for the first pass in the main linac, at the bunch center:

$$
\left\langle x^{2}(z=0)\right\rangle^{1 / 2} \approx 0.0135 \sqrt{\left\langle d_{c}{ }^{2}\right\rangle+7.74\left\langle d_{m}^{2}\right\rangle}
$$

For the baseline parameters, and using 500 and $150 \mu \mathrm{m}$ rms misalignments for the rf cavities and cryomodules respectively [5,6], Equation (10) yields a value of less than $10 \mu \mathrm{m}$ for the rms transverse displacement of the electrons at the bunch center after the first linac pass.

In Figure 4-4 we compare Equation (10) with the tracking code output for several other misalignments values, finding a fairly good agreement.

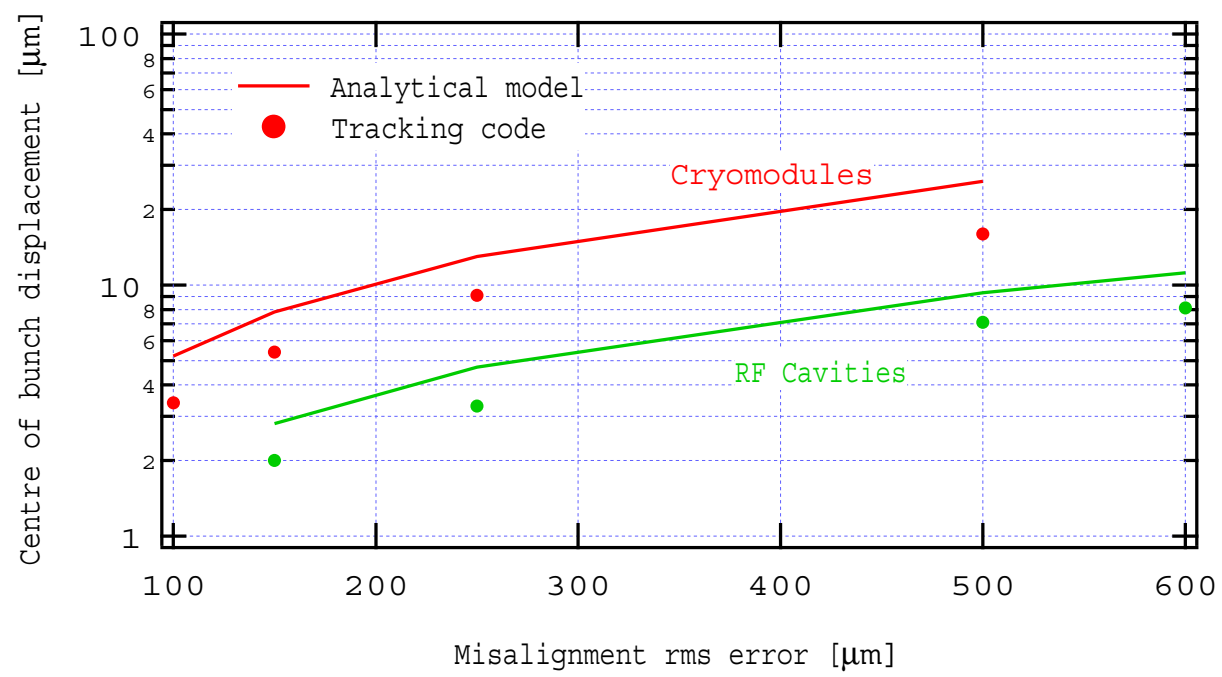

Figure 4-4 RMS value of the transverse displacement at the bunch centre $(z=0)$ as a function of rf cavities and cryomodules misalignments, after the first linac pass.

A direct consequence of Equation (7), when the cryomodule misalignments are taken into account as well, is that it is possible to cancel the effect of misalignments, at the linac exit, by choosing an opportune value for the initial displacement:

$$
x_{0}=-\frac{\sum_{i=0}^{N_{c a v}-1} d_{c i} F_{i}(L)+\sum_{j=0}^{N_{\text {mod }}-1} d_{m j} G_{j}(L)}{\Delta \gamma / \gamma_{i}-\ln \left(1+\Delta \gamma / \gamma_{i}\right)}
$$

This value can only be measured, after the linac has been assembled and aligned, by direct measurements of the beam profile. For baseline machine parameters, we expect the required offset to be in the range of 50 to $100 \mu \mathrm{m}$. 


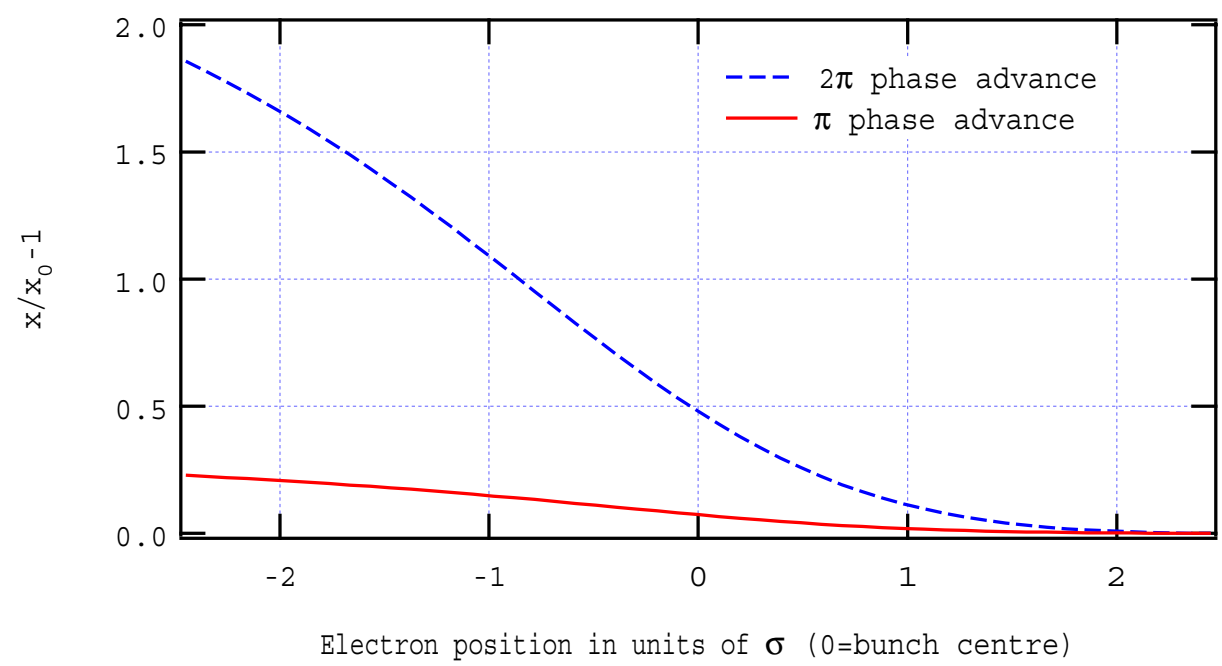

Figure 4-5 Electron displacement for zero betatron phase advance in all arcs (dotted line) and when the first arc has a $\pi$ phase advance, for coordinate displacement $x_{o}$ at the entrance to the linac.

It is also possible to design the arcs in such a way that the subsequent linac passes will have a canceling effect on the (strongest) displacement introduced in the first pass. Figure 4-5 shows the result from the tracking code with a betatron phase advance of $\pi$ in the first return path and $2 \pi$ in the subsequent return paths as opposed to the case of $2 \pi$ phase advance in all four return paths. The results are calculated using a numerical tracking code with a $500 \mu \mathrm{m} \mathrm{rms} \mathrm{displacement} \mathrm{error}$

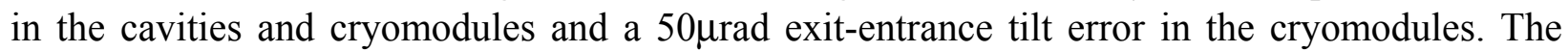
improvement can be explained by the fact that changing the sign of the betatron phase along the bunch after the first turn inverts to displacement of the bunch and results in subsequent wakefields acting to reduce the transverse displacement generated in the first pass.

\section{LONG-RANGE WAKEFIELDS}

Because electron bunches are recirculated several times through the main linac, the effects of long-range wakefields stored in the rf cavity high order modes (HOM) has also been investigated. Data for the TESLA cavities HOM can be found in [5]

In general, the transverse wake field for a resonant mode is given by

$$
w_{\perp}(t)=\frac{2 c}{\bar{\omega}} \frac{k_{i \perp}}{b^{2}} e^{-t / \tau} \sin (\bar{\omega} t)
$$

where $b$ is the beam pipe radius, $k_{i \perp}$ is the transverse kick factor of the $i$-th mode, $\tau=2 Q / \omega$ and $\bar{\omega}=\sqrt{\omega^{2}-1 / \tau^{2}}$.

The total transverse wake field is simply the sum of contributions from all the HOMs: 


$$
w_{\perp, t o t}(t)=\sum_{i=1}^{N_{\text {hom }}} \frac{2 c}{\bar{\omega}_{i}} \frac{k_{i \perp}}{b^{2}} e^{-t / \tau_{i}} \sin \left(\bar{\omega}_{i} t\right)
$$

Given the high $Q$ values, even for the damped modes, of order $10^{4}$, with filling times of order microseconds, this field is not appreciably damped in the time it takes a bunch to re-enter the linac $(<1 \mu \mathrm{s})$. The wakefields from different passes add up, and we can write the total wakefield at time $t$, with $T_{p}$ the difference between the current time $t$ and the time of the $\mathrm{p}^{\text {th }}$ passage:

$$
w_{\perp, t o t}(t)=\sum_{i=1}^{N_{\text {hom }}} \frac{2 c}{\bar{\omega}_{i}} \frac{k_{i \perp}}{b^{2}} \sum_{p=1}^{N_{\text {pass }}} e^{-\left(t+T_{p}\right) / \tau_{i}} \sin \left[\bar{\omega}_{i}\left(t+T_{p}\right)\right]
$$

Figure 4-6 shows the typical long range transverse wake after a bunch first passage in the linac. A random detuning of $\pm 0.1 \%$ between cavities has been taken into account, in the same way as done in the TESLA design report [5]. On its second pass the bunch will experience this wake value for $t=T_{1}$ and leave an identical wake, shifted in time by $t=T_{1}$. The sum of these two wakes, calculated in $t=T_{2}$, is the wake experienced by the bunch on its third pass and so on.

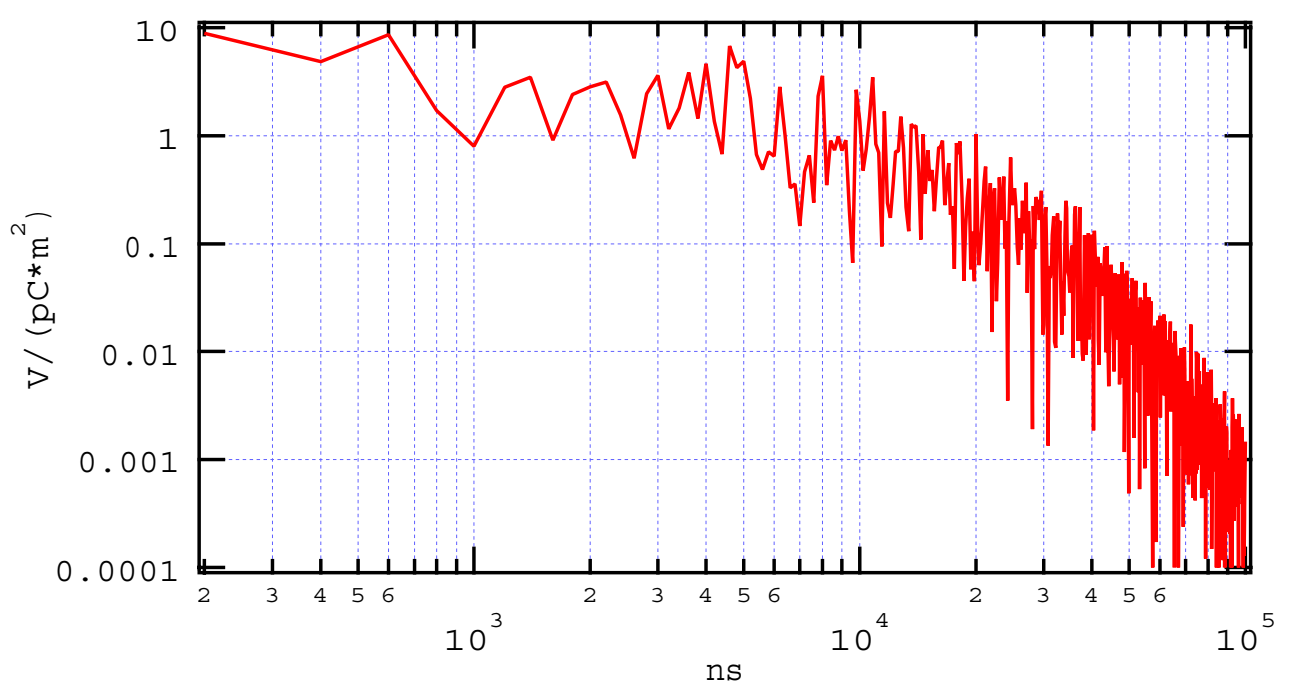

Figure 4-6 Transverse long-range wake averaged over 32 cavities with a frequency spread of $\pm 0.1 \%$.

Since the single-pass wakefield oscillates rapidly, its amplitude, as experienced by a bunch, depends on the exact time of passage. Even though later passes experience a sum of the wakes left from all the preceding passes, the oscillations in amplitude make it so that a bunch is not guaranteed to encounter a higher wake on a later pass, rather than on a previous one. We can see that the worst case is still that of the lowest energy pass, where the bunch is most sensitive to the transverse wake action. 
The wake has sufficient time to decay to a negligible level when bunches are injected at the $10 \mathrm{kHz}$ rate, and the long-range wake amplitude is fairly constant over the design bunch length of a few picoseconds.

Figure 4-7 shows a comparison of typical short and long range transverse wakefields for a 2 ps long bunch. It can be seen that the long range wake results in a small offset of the entire bunch. This also indicates that the amplitude of the long-range transverse wakefield is, generally, much smaller than the short-range one.

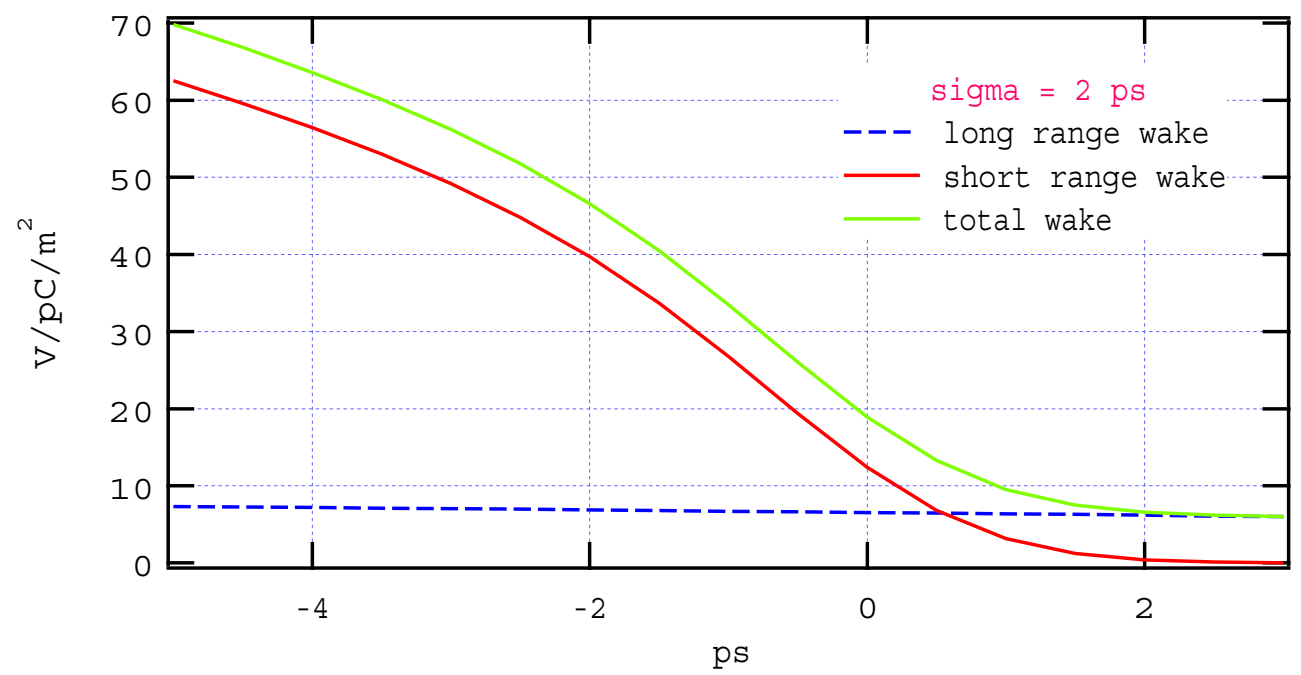

Figure 4-7 Short and long range transverse wakefields for a 2 ps long bunch.

\section{INJECTOR LINAC}

Before injection into the main linac, the electron bunch is accelerated from 10 to $120 \mathrm{MeV}$ in an injector linac. This pre-accelerator is composed of a single cryomodule of the same kind as in the main linac. Therefore we will assume that the wake fields are the same and use the same formulae already derived for the main linac, but with different linac length $(8.0 \mathrm{~m})$, injection energy and energy gain. An important difference with the main linac is that, due to the lower energy, the bunch is subject to the focussing effect from the RF field [7]. This effect is included in Equation (1), when there are no other focussing elements, by writing:

$$
k^{2}=\frac{1}{2}\left(\frac{r_{0} G}{e \gamma}\right)^{2}
$$

where $G \approx 14 \mathrm{MV} / \mathrm{m}$ is the average accelerating gradient in the pre-accelerator.

Given the high relative energy change of a factor 12 from injection to extraction, the betatron wavelength changes from $4.83 \mathrm{~m}$ at injection to $31.4 \mathrm{~m}$ at the end of the injector linac.

Under these conditions, Equation (1) can no longer be solved analytically. Therefore, we check that in the absence of focussing the wake field effect is still below the maximum allowed. This will be a conservative estimate since focussing can only help control the BBU growth. 
From Equation (4) it is readily seen that the bunch center transverse displacement at the end of the linac, for a 2 ps bunch, is

$$
\frac{x(z=0)}{x_{0}} \approx 2.410^{-3}
$$

As, this is a small effect, it may be ignored.

\section{LONGITUDINAL WAKEFIELD}

The voltage associated with the longitudinal wakefield in the main linac can introduce energy spread within a bunch. A classic remedy to this effect is accelerating the bunch off the rf wave crest, choosing an appropriate phase such that the variation of the main rf voltage along the bunch counterbalances the wakefield voltage.

We can write the rf voltage experienced by a particle inside the bunch as:

$$
V_{R F}\left(\varphi_{0}, \tau\right)=\hat{V}_{R F} \cos \left(\varphi_{0}+\omega_{R F} \tau\right)
$$

where $\varphi_{0}$ is the bunch centre phase, with respect to the rf wave, $\hat{V}_{R F}$ is the RF peak voltage and $\omega_{R F}$ its angular frequency, $\tau$ indicates a particle position inside the bunch, $\tau=0$ corresponding to the bunch center.

The voltage from the longitudinal wakefield is written as:

$$
V_{W}(s)=\int_{-\infty}^{s} \rho\left(s^{\prime}\right) w\left(s-s^{\prime}\right) d s^{\prime}
$$

where $\rho(s)$ is the bunch charge distribution, $w(s)$ the longitudinal wake function and $s$ is the distance from the bunch center.

Since $s=c \tau$, the total voltage along the bunch is

$$
V_{t o t}\left(\varphi_{0}, \tau\right)=V_{R F}\left(\varphi_{0}, \tau\right)+V_{W}(\tau)
$$

From its Taylor series expansion, about the bunch centre

$$
\begin{aligned}
& V_{\text {tot }}\left(\varphi_{0}, \tau\right) \approx V_{\text {tot }}\left(\varphi_{0}, 0\right)+\frac{\partial}{\partial \tau}\left[V_{t o t}\left(\varphi_{0}, \tau\right)\right]_{\tau=0} \tau= \\
& \quad=\hat{V}_{R F} \cos \varphi_{0}+V_{W}(0)-\left(\omega_{R F} \hat{V}_{R F} \sin \varphi_{0}-\frac{\partial}{\partial \tau}\left[V_{W}(\tau)\right]_{\tau=0}\right) \tau
\end{aligned}
$$

we see that canceling the term in between brackets would cancel the first-order dependence of the total voltage on the position inside the bunch. This means choosing a bunch center phase such that

$$
\sin \varphi_{0}=\frac{c}{\omega_{R F} \hat{V}_{R F}} \frac{\partial}{\partial s}\left(\int_{-\infty}^{s} \rho\left(s^{\prime}\right) w\left(s-s^{\prime}\right) d s^{\prime}\right)_{s=0}
$$

To calculate the integral in Equation (21) we use the following wake function:

$$
w(s)=-38.1\left\lfloor\frac{V}{p C \cdot m}\right\rfloor\left(1.165 e^{-\sqrt{s \mid 3.65 m m}}-0.165\right)
$$

given in [5] for the steady-state wake of a point charge. The use of the steady-state wake is justified for the main linac, but it is only approximately correct in the case of the shorter injector 
linac. The uniform longitudinal charge distribution we use is defined as $\lambda(s)=Q_{b} / l_{b}$ in the interval $0<s<l_{b}$, where $Q_{b}$ is the bunch charge and $l_{b}$ its length.

With these assumptions, Equation (21) yields an absolutely negligible value for the required rf phase, demonstrating that the longitudinal wakefield effect can be ignored in the main linac. For the case of the injector we find that timing jitter following the action of the bunch compressor require correction of the longitudinal wake effects in the injector linac up to the second order. To this end, using Equation (22) and the charge distribution defined above we calculate the electrons energy loss, as a function of their position in the bunch, using the following expression:

$$
\frac{1}{Q_{b}} \frac{d E(s)}{d z}=-19.05\left[\frac{V}{p C \cdot m}\right] \int_{-1}^{2 s / l_{b}}\left(1.165 e^{-\sqrt{\frac{s-x\left(l_{b} / 2\right)}{3.65 m m}}}-0.165\right) d x
$$

We found that the right hand side of Equation (23) can be accurately described by a quadratic polynomial. For the injector linac, with a bunch length of 20 ps we obtain:

$$
\frac{1}{Q_{b}} \frac{d E(s)}{d z}=\left\lfloor-9.32-6.34\left(\frac{2 s}{l_{b}}\right)+2.42\left(\frac{2 s}{l_{b}}\right)^{2}\right]\left[\frac{V}{p C \cdot m}\right]
$$

and for the main linac, with a 2 ps bunch length, we obtain:

$$
\frac{1}{Q_{b}} \frac{d E(s)}{d z}=\left\lfloor-15.25-13.72\left(\frac{2 s}{l_{b}}\right)+1.33\left(\frac{2 s}{l_{b}}\right)^{2}\right\rfloor\left[\frac{V}{p C \cdot m}\right]
$$

Figure 8 shows plots of the two fitting functions given in Equations (25) and (25) (colored dashed curves). We also evaluate directly Equation (23) for the same bunch lengths (solid black curves) and found that the results are virtually indistinguishable.

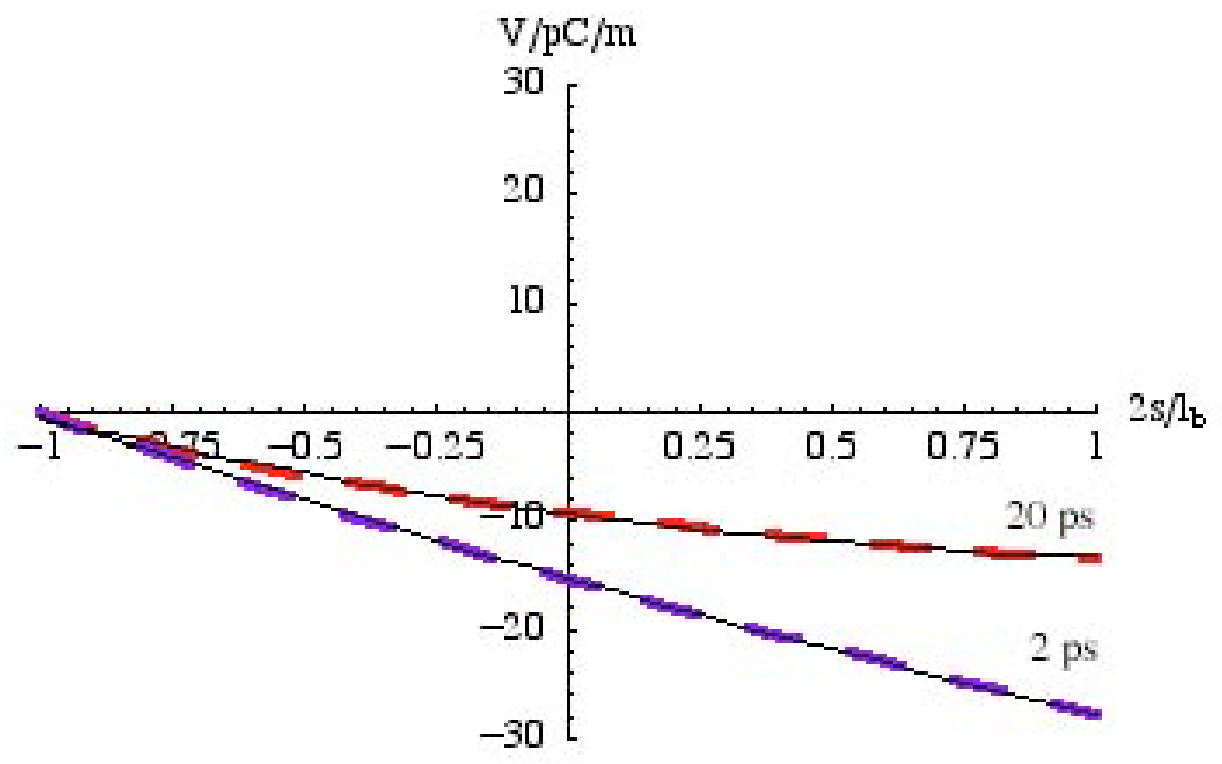

Figure 4-8 Energy loss of electrons, due to the longitudinal wake, for 2 ps and 20 ps bunches. 


\section{Tracking STUdies}

In addition to analytical estimates a more complete investigation including the nominal bunch distribution and tracking through the arcs has been made using a tracking code. For the present studies, we have used MERLIN [8], which allows the simulation of all the required effects.

\section{Linac Tracking}

The present design of the linac uses the TESLA 9 cell, $1.3 \mathrm{GHz}$ superconducting cavities [4]. These are standing wave structures, for which the transfer map (the Chambers' Matrix) is known [9]. The fringe-field focusing is included in this transfer map, and although the exit field of one cavity is (approximately) cancelled by the entrance field of the following cavity, the exit and entrance fields of the whole linac do not cancel because of the gain in energy through the linac. This effect is most significant in the first pass through the linac, since the relative gain in energy is large. There is an effect on the linear optics from the fringe-field focusing; this has been included in the lattice design produced using MAD 8acc [10]. The implementation of the transfer map in MERLIN differs in one respect from that in MAD 8acc, namely the MERLIN map includes a transverse focusing from the RF in the body of the cavity, which is negligibly small in the parameter regime (cavity parameters and beam energy) of the femtosecond light source. This term is omitted in the MAD 8acc map [11]. The Chambers' Matrix gives the correct adiabatic damping of the transverse and longitudinal emittances.

It is assumed that the orbit through the linac can be controlled to the level of a few tens of microns. The main contribution to the wake fields then comes from misalignments of the cavities. The specified vertical alignment tolerance of the cavities in the linac is $500 \mu \mathrm{m}$ rms within a cryostat, and $150 \mu \mathrm{m} \mathrm{rms} \mathrm{between} \mathrm{cryostats.} \mathrm{The} \mathrm{lattice} \mathrm{model} \mathrm{is} \mathrm{constructed} \mathrm{in}$ MERLIN in such a way that the beam sees the same set of misalignments on each pass through the linac.

The wake fields are implemented in the standard way for macroparticle tracking codes. Specifically, the macroparticles are binned to determine the longitudinal distribution, and an appropriate kick is determined for each macroparticle by forming the convolution of the distribution with the specified Green's function. In MERLIN, any number of steps can generally be taken through beamline components, with processes such as wake fields applied at the end of each step. However, a limitation specific for a standing wave cavity, is that the transfer map is valid only for a cavity consisting of an integer number of cells. In the present simulation, each cavity is treated as a sequence of nine cells with zero separation between; wake field kicks were therefore applied nine times for each cavity.

\section{Arc Tracking}

Symplectic tracking is implemented for standard (non-accelerating) beamline components. Nonlinear components such as sextupoles can be treated in various approximations; for the present studies, they were treated in the thin-lens approximation. The symplectic condition ensures that no spurious emittance growth or damping is introduced. 
The lattice design uses sextupoles to minimize the second-order dependence of time-of-flight on energy. Although the sextupole strengths are not great, vertical beam offset in the sextupoles could have a significant impact on machine performance through the coupling of horizontal emittance into the vertical plane. Even if the beam was injected on the design orbit into the linac for these simulations, the fringe fields and wake fields from misaligned cavities lead to orbit distortion. To separate the emittance growth from wake fields and sextupoles, we simply compare the results of tracking with and without sextupoles. In the latter case, there is an effect on the bunch length, with a longitudinal tail that is not expected to affect the results significantly. Note that the map for a dipole implemented in MERLIN includes second-order terms that can also give betatron coupling; some vertical emittance growth is therefore expected even with the sextupoles turned off, though this should be much less than the coupling resulting from the sextupoles. Below, the emittance growth in several situations, with wake fields and sextupoles turned on and off in various combinations, is compared.

The arcs include a phase trombone to allow control over the betatron phase advances. It is possible, for example, that there may be benefits to having half-integer phase advances over each arc. For the present studies, although the arcs were not specially tuned, the vertical phase advance over each arc was close to a half integer. This means that the wake field kicks on successive passes will nearly cancel.

\section{Benchmarking Wake Field Simulations}

To verify the wake field model in MERLIN, a bunch with nominal 2 ps bunch length, and (effectively) zero transverse emittance was first tracked through four passes of the linac. In this case, the bunch was injected so as to have a fixed vertical offset in each cavity, and the bunch was taken straight from the end of the linac on each pass and re-injected at the start of the linac: i.e. modeling the effects of perfectly achromatic arcs with integer betatron phase advances. This system has been studied semi-analytically and reported earlier, and provides a benchmark for the wake field model in our MERLIN simulations.

The results of the simulation are shown in Figure 4-9, tracking a bunch with the nominal 1 $\mathrm{nC}$ charge, consisting of 10,000 macroparticles: the macroparticles are somewhat sparse at the 3 sigma tails of the distribution. The vertical axis is normalized, so that the transverse deflection is expressed as a fraction of the initial bunch offset. In the previous analysis, it is assumed that the length of the linac is filled with structures, whereas the packing factor is actually about $80 \%$. Once this factor is taken into account, there is excellent agreement between the results of Figure 1 and the results shown in Figure 4-9.

It should be noted that the 35\% deflection after four passes observed in Figure 4-9 represents an extremely pessimistic case. With random misalignments, there will be significant cancellation between the wake fields of different cavities (aside from pathological cases). In addition, the phase advance over the arcs mentioned above will tend to cancel the effects of the kicks on successive passes. 


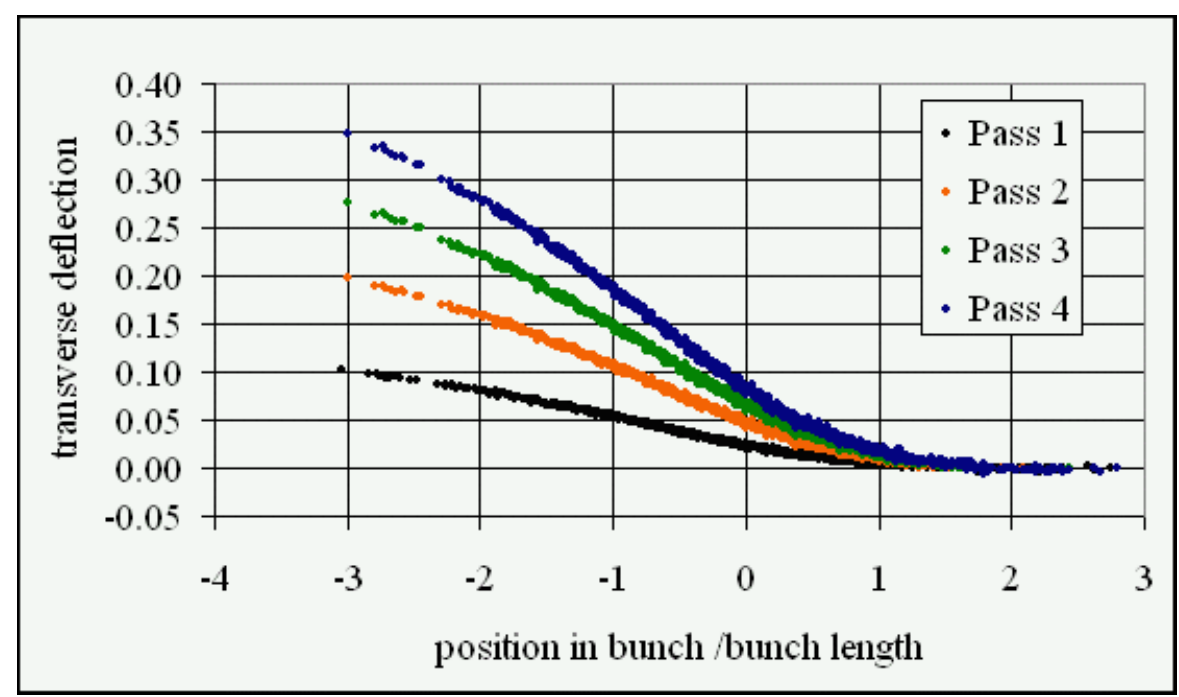

Figure 4-9 Transverse deflections of a 2 ps zero-emittance beam, after four consecutive passes at constant vertical offset through the linac.

\section{Vertical Emittance Growth}

To estimate the emittance growth from the wake fields in a linac with vertical cavity misalignments, a bunch (of 10,000 macroparticles) was tracked with the nominal parameters as specified in Table 4-1 from the entrance to the linac to the exit after the fourth pass. Vertical misalignments with a range of rms values (and a cut-off at 5 sigma) were applied to the cavities; as mentioned above, the beam sees the same misalignments in the simulation on each pass. The cavity misalignments were the only imperfections applied to the machine; the arc optics and alignment were as designed. For large cavity misalignments, there is significant orbit distortion that is expected to lead to vertical emittance growth from coupling in the arc sextupoles. No distinction was made between cryostats; in reality, cavity offsets are expected to be correlated according to which cryostat they are in. Since we find that the wake field effects for any reasonable cavity misalignments are so small, our conclusions are not likely to be affected by the fact that we have neglected this effect.

Table 4-1 Bunch parameters used in wake field simulations

\begin{tabular}{|c|c|}
\hline Initial bunch energy & $120 \mathrm{MeV}$ \\
\hline Bunch charge & $1 \mathrm{nC}$ \\
\hline Bunch length & $2 \mathrm{ps}$ \\
\hline Energy spread & $10^{-3}$ \\
\hline Horizontal emittance (normalized) & $20 \mu \mathrm{m}$ \\
\hline Vertical emittance (normalized) & $0.4 \mu \mathrm{m}$ \\
\hline
\end{tabular}


Results of tracking with the sextupoles turned on are shown in Figure 4-10; results with the sextupoles off are shown in Figure 4-11. Each figure shows the results of 200 seeds (the same seeds for each data series) of cavity misalignments with rms varying from zero up to $2 \mathrm{~mm}$. Note the different vertical scales in the two graphs, and that the vertical misalignment scale (horizontal axis) extends an order of magnitude beyond the specified alignment tolerance on the cavities.

It appears that the wake fields make negligible contribution to the emittance growth as can be shown with a simple estimate based on the results shown in Figure 4-9. For the mean vertical displacement we take the largest rms cavity misalignment divided by the square root of the number of cavities in the linac (the linac is composed of 32 cavities). The displacement between the head (+1 sigma) and the tail (-1 sigma) of the bunch after the fourth pass, given no phase advance over the arcs, is of the order $15 \%$ of the mean cavity misalignment. For $2 \mathrm{~mm} \mathrm{rms}$ (and hence $350 \mu \mathrm{m}$ mean) cavity misalignment, we expect a vertical displacement of the bunch tail with respect to the head of about $50 \mu \mathrm{m}$. This is to be compared with the bunch size of $70 \mu \mathrm{m}$ at the end of the fourth pass of the linac. The wake field cancellation resulting from the phase advance across the arcs can easily reduce the head-tail displacement by more than an order of magnitude; in which case, we would expect to see an emittance growth of the order 5\% (with 2 $\mathrm{mm}$ rms vertical cavity misalignment). From Figure 4-11, it appears the wake fields make an even smaller contribution to the vertical emittance; we note that the mean cavity misalignment in our simulations is actually closer to $100 \mu \mathrm{m}$ than $350 \mu \mathrm{m}$ (statistical fluctuations) when the rms cavity misalignment is around $2 \mathrm{~mm}$.

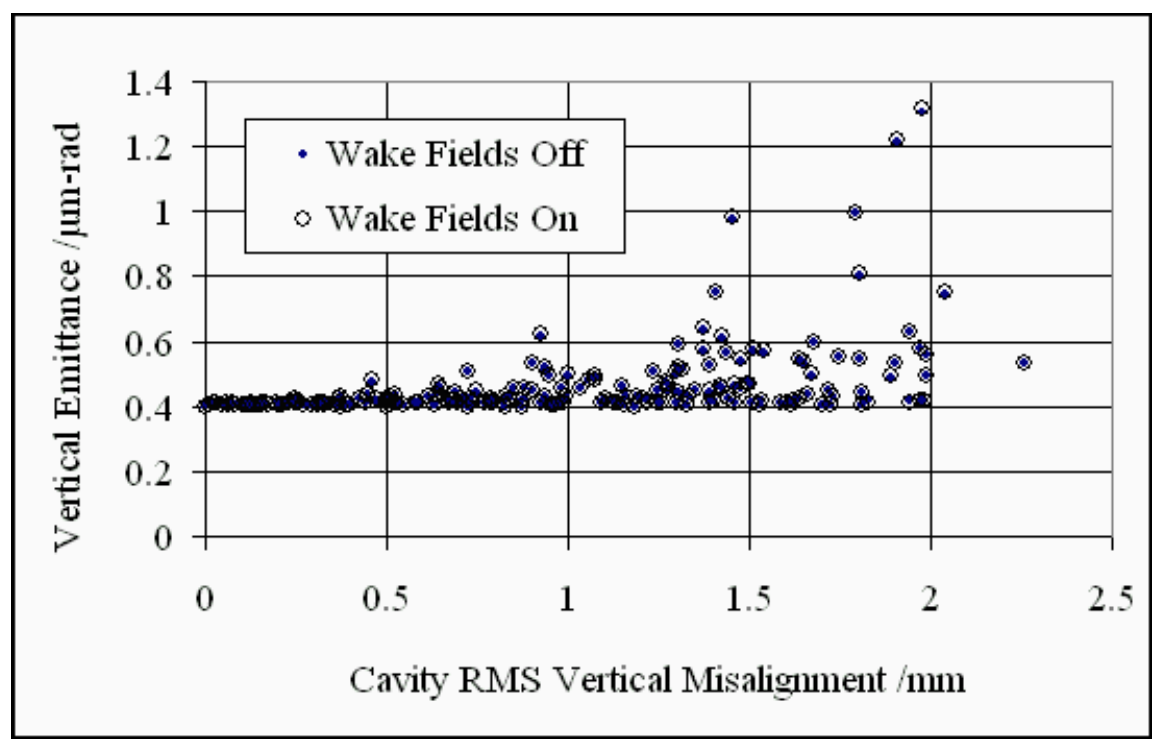

Figure 4-10 Results of tracking a nominal bunch from the first entrance of the linac through four passes (including the arcs) to the fourth exit from the linac. The arc sextupoles were turned on. 


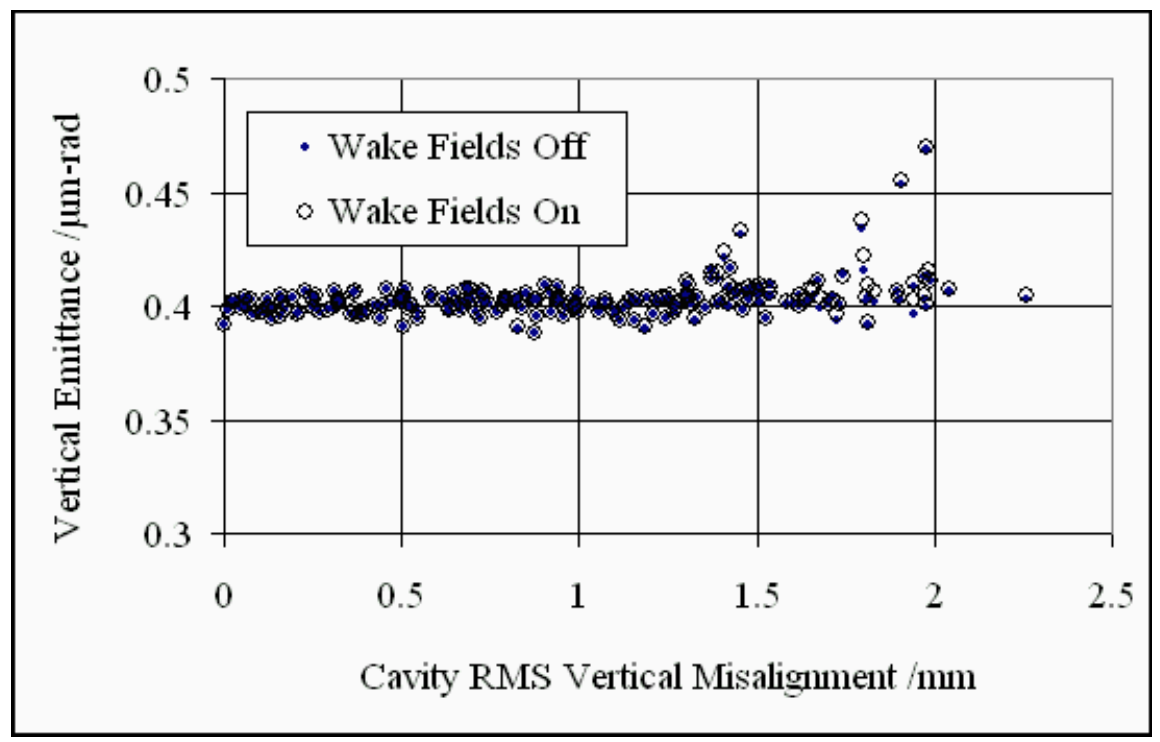

Figure 4-11 Results of tracking a nominal bunch from the first entrance of the linac through four passes (including the arcs) to the fourth exit from the linac. The arc sextupoles were turned off.

With the sextupoles turned on, any emittance growth from the wake fields is hidden by the very much larger emittance growth from the coupling. This should be easily fixed by proper orbit control.

The results of our simulations of emittance growth resulting from wake fields in the $\mathrm{rf}$ cavities are broadly consistent with rough estimates, based on knowledge of the wake field effects in the case of a constant beam transverse offset in the linac. For any reasonable cavity alignment (a few hundred microns rms with respect to the design orbit), the emittance growth from wake fields in the cavities is small. Controlling the orbit in the sextupoles to avoid emittance growth from betatron coupling is a more significant issue.

Figure 4-12 shows how the vertical-longuitudinal correlation, introduced by the wake fields, is negligible even when no coupling is present (sextupoles off).

\section{RESISTIVE WALL IMPEDANCE}

The transverse wakefield from the resistive wall impedance for a circular pipe of radius $b$, length $L$, electrical conductivity $\sigma_{\mathrm{c}}$ is given in $V / p C / m$ by [12]:

$$
W_{1}(z)=\frac{c L}{\pi b^{3}} \sqrt{\frac{Z_{0}}{\pi \sigma_{c}}} \frac{1}{\sqrt{z}}
$$

For a charge distribution $\rho\left(z^{\prime}\right)$ the wake is then:

$$
W_{1}(z)=\frac{c L}{\pi b^{3}} \sqrt{\frac{Z_{0}}{\pi \sigma_{c}}} \int_{-\infty}^{z} \rho\left(z^{\prime}\right) \frac{d z^{\prime}}{\sqrt{\left|z-z^{\prime}\right|}}
$$




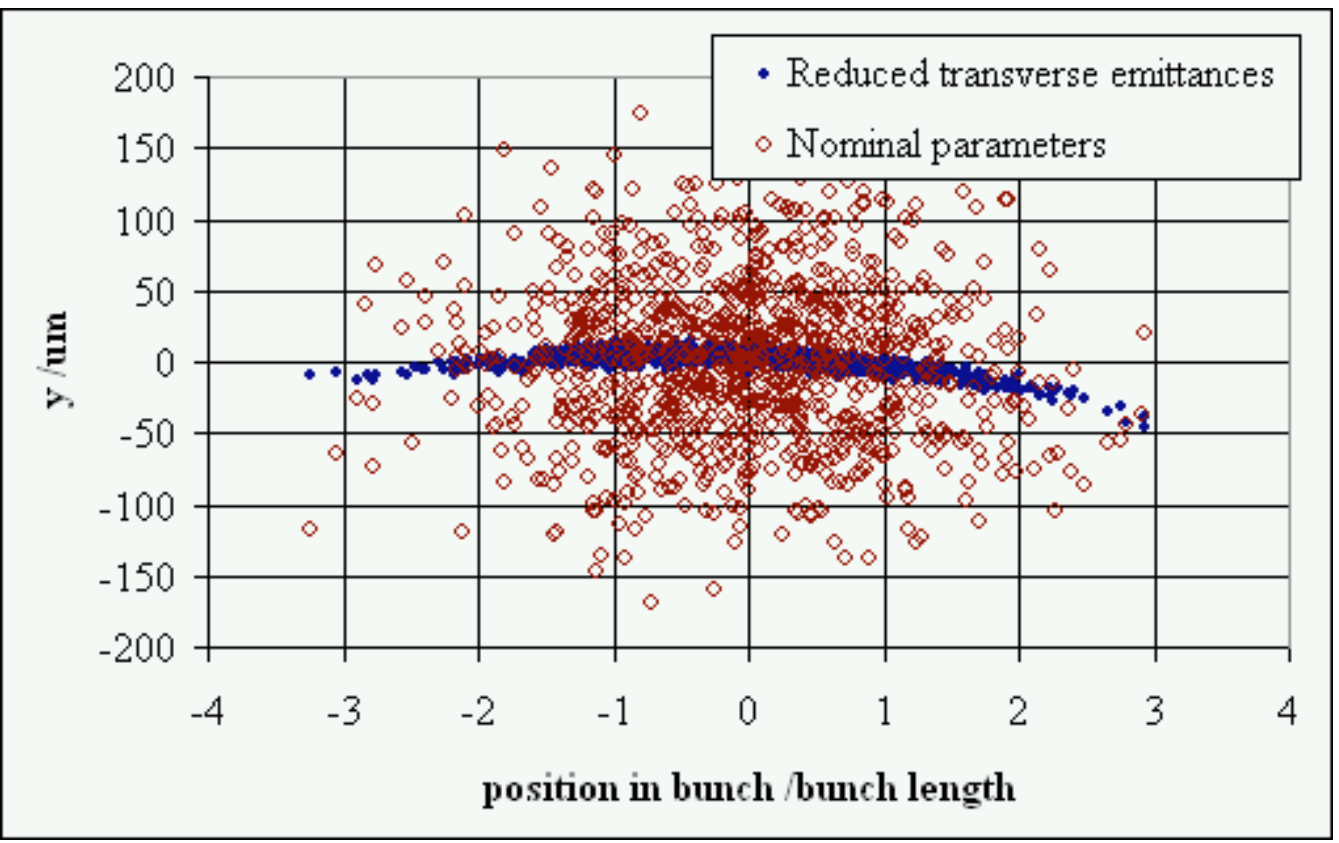

Figure 4-12 Results of tracking two bunches four passes through the linac and arcs. The cavities were misaligned with rms $2 \mathrm{~mm}$ and mean $350 \mathrm{~mm}$, and the sextupoles were turned off. The vertical-longitudinal correlation introduced by the wake fields is visible when the transverse emittances are reduced (by six orders of magnitude), but the effect on the nominal bunch is negligible.

which, for a Gaussian bunch of unit charge becomes:

$$
W_{1}(z)=\frac{c L}{\pi b^{3}} \sqrt{\frac{Z_{0}}{\pi \sigma_{c}}} \frac{1}{\sigma_{z} \sqrt{2 \pi}} \int_{-\infty}^{z} e^{-\frac{z^{2}}{2 \sigma_{z}^{2}}} \frac{d z^{\prime}}{\sqrt{\left|z-z^{\prime}\right|}}
$$

The deflecting voltage for a bunch of $\mathrm{N}$ particles of charge $e$ and offset $y_{0}$ is therefore:

$$
V_{\perp}(z)=N \text { e } y_{0} W_{1}(z)
$$

The angular deflection along a gaussian beam of energy $E$ is given by:

$$
\Delta y^{\prime}(z)=\frac{N e y_{0}}{\frac{E}{e}} W_{1}(z)
$$

Note that the resistive wall induced kick scales inversely with energy and the beamsize scales inversely with the square root of energy, thus the increase in projected emittance resulting from wakefield-induced distortions along the bunch is largest at low-energy.

Starting from the parameters of Table 4-2, wakefields are calculated solving the above expressions numerically for Gaussian bunches and similar expressions for a rectangular charge distribution. The resulting transverse deflection along the bunch is calculated for a unit length of beampipe. 


\section{Table 4-2 Baseline parameters for resistive wall calculations}

\begin{tabular}{|c|c|}
\hline Normalized vertical emittance & $0.4 \mathrm{~mm}$-mrad \\
$\gamma \varepsilon_{\mathrm{v}}$ & $10-30 \mathrm{~m}$ \\
$\beta$-function $\beta_{\mathrm{v}}$ & Beamsize $\sigma_{\mathrm{v}}$ \\
\hline Beam energy (pass \#) & $170-511 \mu \mathrm{m}$ \\
$120 \mathrm{MeV}(0)$ & $54-93 \mu \mathrm{m}$ \\
$710 \mathrm{MeV}(1)$ & $40-69 \mu \mathrm{m}$ \\
$1.3 \mathrm{GeV}(2)$ & $33-57 \mu \mathrm{m}$ \\
$1.9 \mathrm{GeV}(3)$ & $28-50 \mu \mathrm{m}$ \\
$2.5 \mathrm{GeV}(4)$ & $1.5 \mathrm{~cm}$ \\
Chamber radius & $1 \mathrm{~m}$ \\
Chamber length & $3.5 \times 10^{7}(\mathrm{Al})$ \\
Chamber conductivity & $100 \mu \mathrm{m}$ \\
Bunch offset & $6.24 \times 10^{9}$ \\
\hline
\end{tabular}

We choose here aluminum vacuum chambers, although other materials may be considered, for example a copper $\left(\sigma_{\mathrm{Cu}}=5.6 \times 10^{7}\right)$ vacuum chamber reduces the wakefield by $25 \%$. A stainless steel $\left(\sigma_{\text {St. St. }}=0.11 \times 10^{7}\right)$ vacuum chamber, however, increases the effect by a factor 5.6.

\section{First arc (710 MeV)}

Figure 4-13 shows the resistive wall wakefield together with the charge distributions for gaussian and rectangular bunches in the first full arc at $710 \mathrm{MeV}$. The deflection angles for the same parameters are shown in Figure 4-14. These figures show that the typical "banana" shaped distribution along the bunch resulting from the short-range resistive wall wakefield. Figure 4-15 shows the deflection along a bunch as a function of the bunch length.

The displacement $y$ along the bunch is calculated as the product of the deflection and the length of beampipe. Even for the case of a 1 ps rectangular bunch at $710 \mathrm{MeV}$, the deflection is $6.8 \times 10^{-10} \mathrm{rad} \mathrm{m}^{-1}$ and the distortion at the end of a $2 \mathrm{ps}, 1 \mathrm{nC}$, rectangular bunch arising from resistive wall transverse wakefields is $0.25 \%$ of the vertical beamsize for a complete arc of 200$\mathrm{m}$ length. For a $2 \mathrm{ps}$ bunch at $3 \mathrm{nC}$, the distortion is $0.5 \%$

The bunch distortion from the resistive wall wakefield is small in a $3 \mathrm{~cm}$ diameter aluminum beampipe under these conditions. For a $2 \mathrm{ps}, 3 \mathrm{nC}$, bunch and allowing for $\pm 250 \mu \mathrm{m}$ closed orbit distortion, the vacuum chamber vertical alignment with respect to the quadrupole centers must be better than $1 \mathrm{~mm}$. Wakefields arising from positive and negative offsets will tend to cancel each other, and an average integrated vacuum chamber (vertical) alignment of $1 \mathrm{~mm}$ gives a 5\% distortion within a bunch after the first pass. 


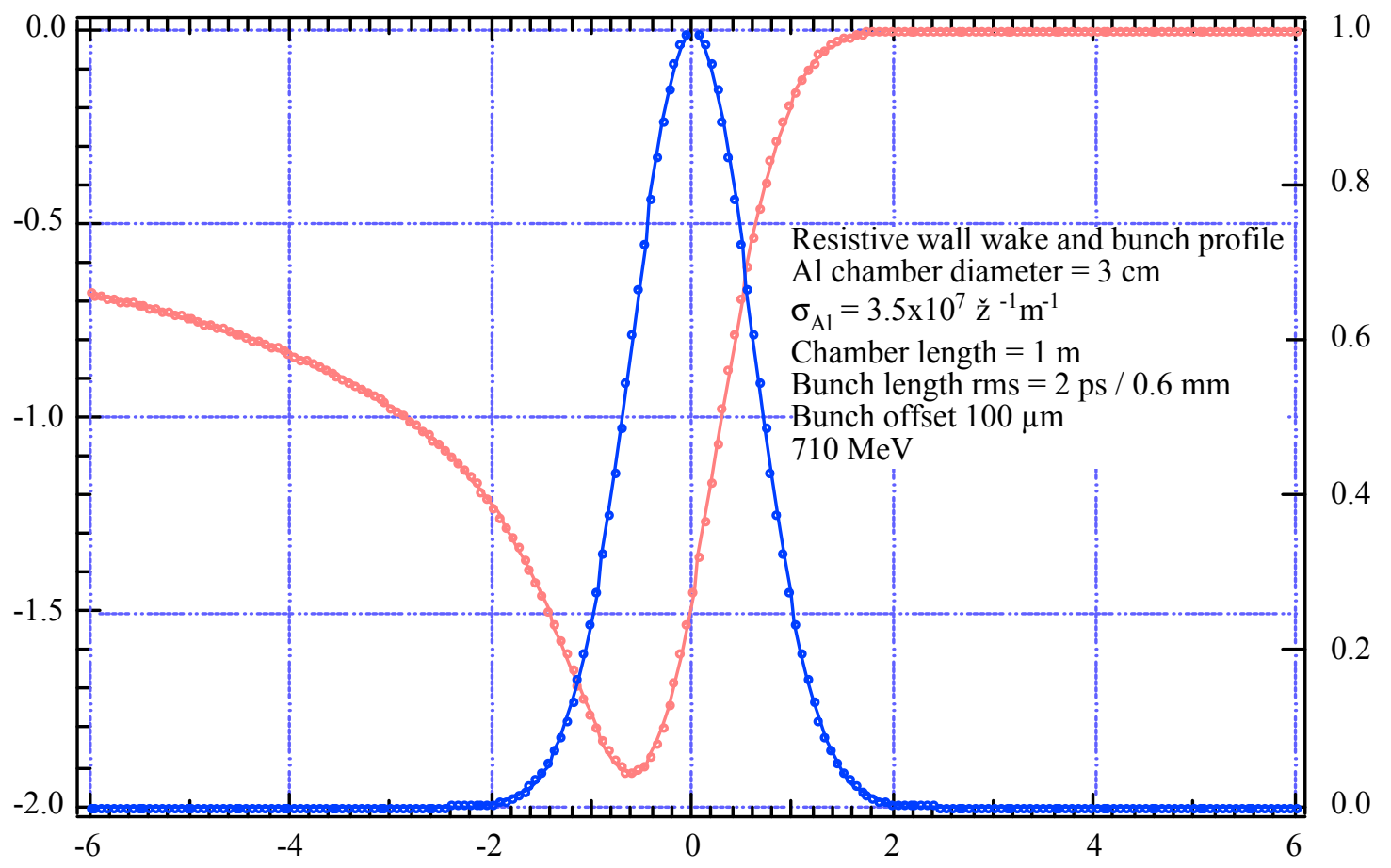

Position relative to bunch center, $\mathrm{mm}$

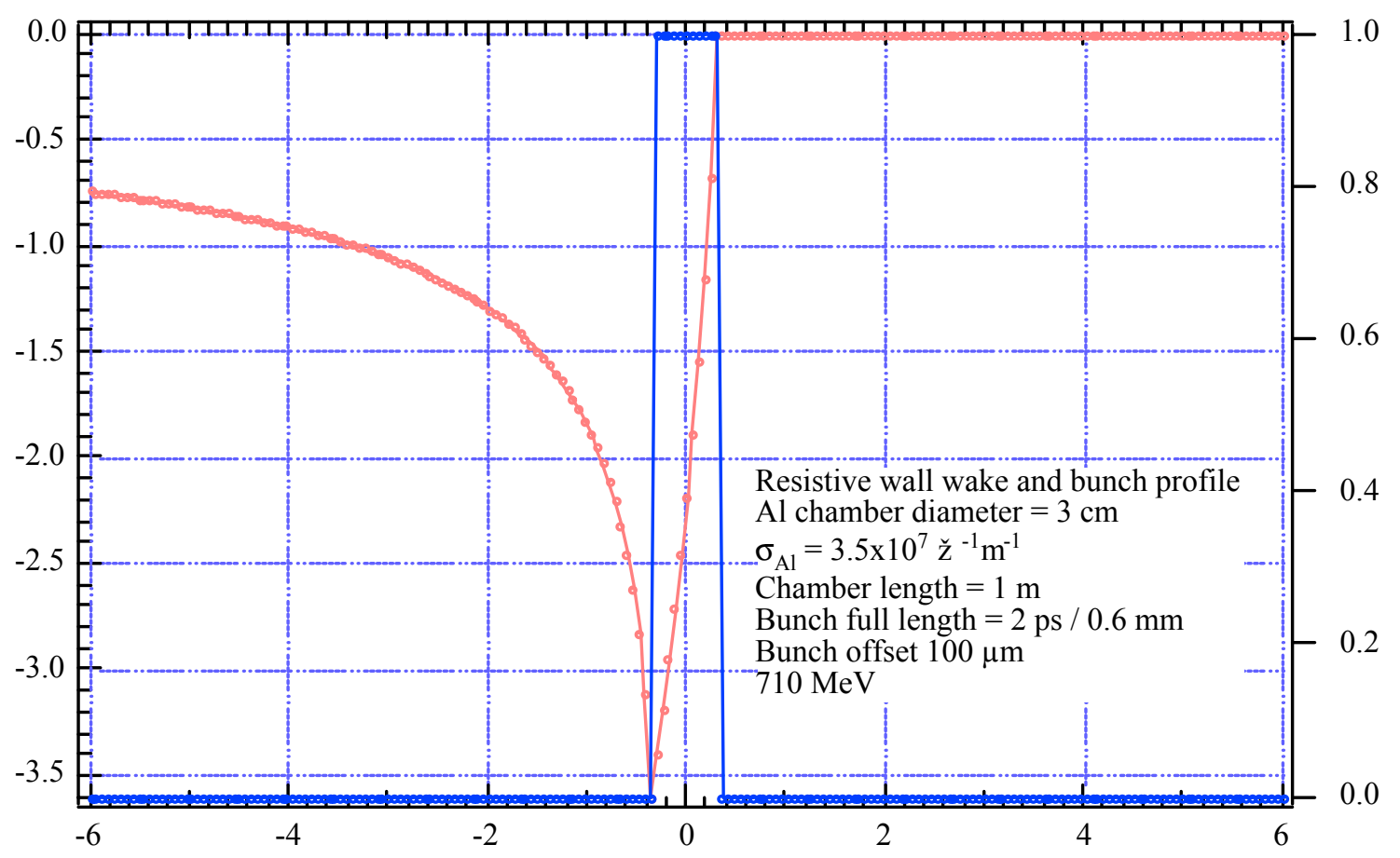

Position relative to bunch center, $\mathrm{mm}$

Figure 4-13 Charge distribution and resistive wall wake in the first arc, for gaussian (top), and rectangular (bottom) longitudinal distributions. 

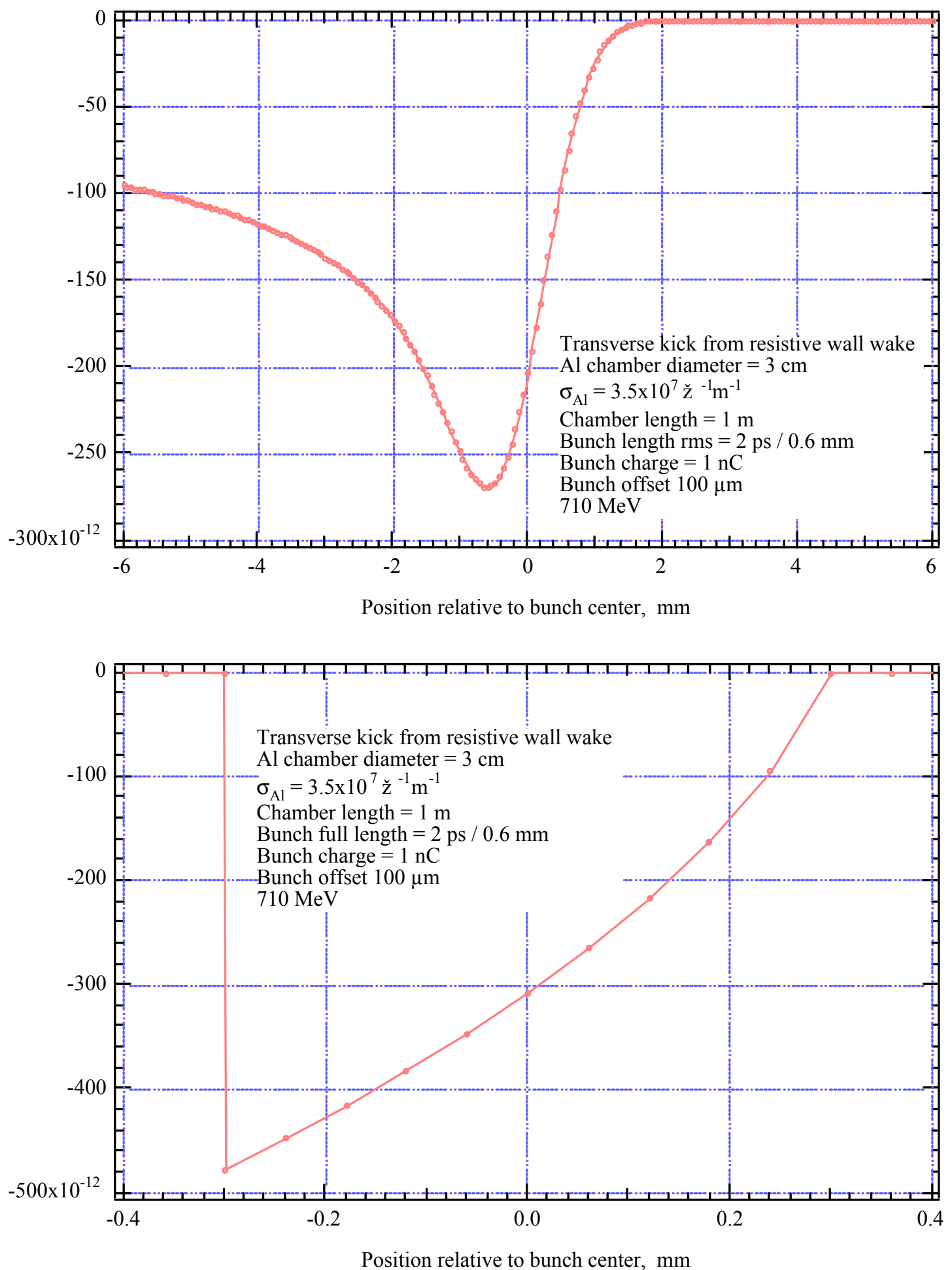

Figure 4-14 Transverse deflection (rad) for a 1-m long, 3-cm diameter vacuum chamber section in the first arc, for gaussian (top), and rectangular (bottom) longitudinal distributions. 


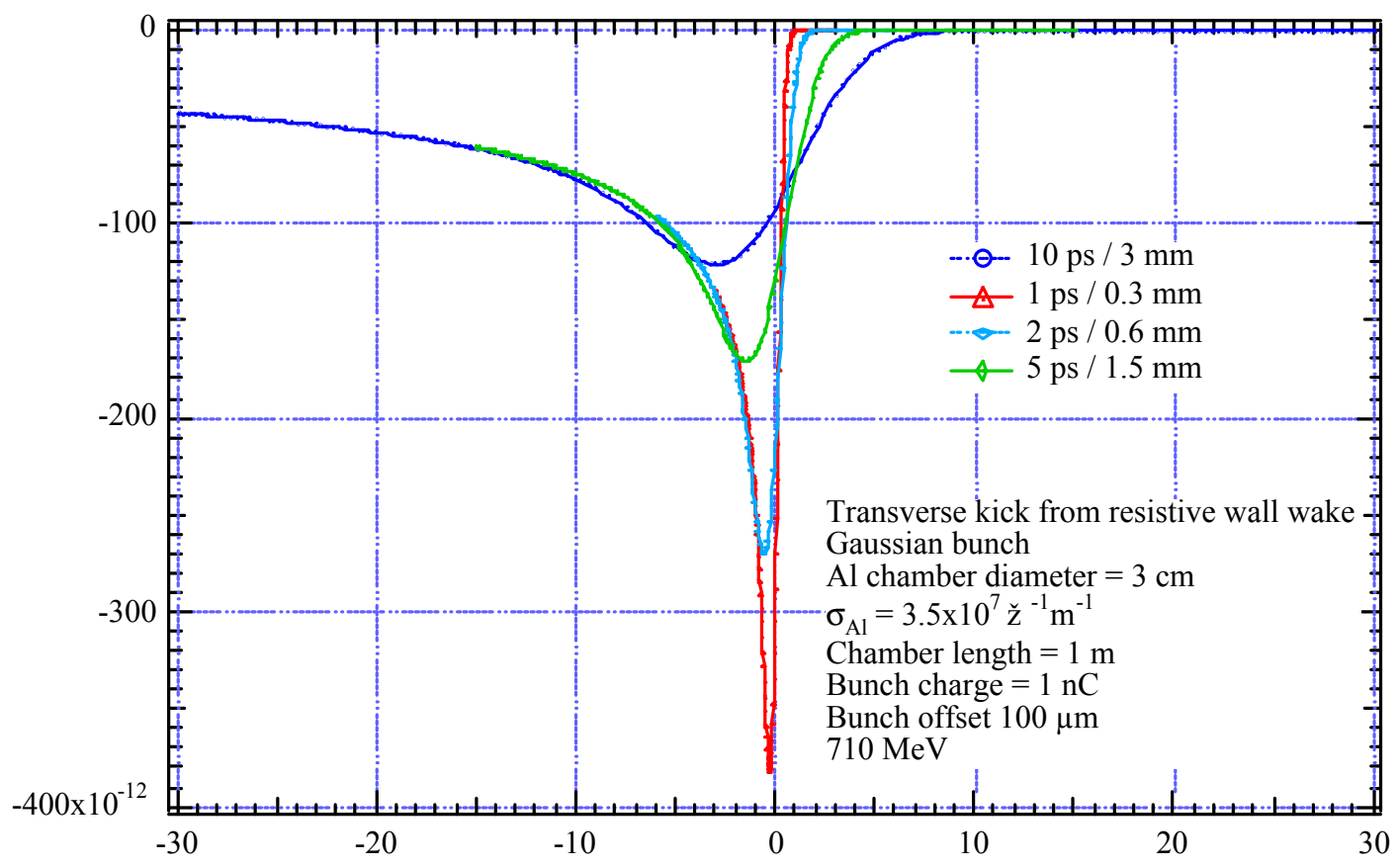

Position relative to bunch center, $\mathrm{mm}$

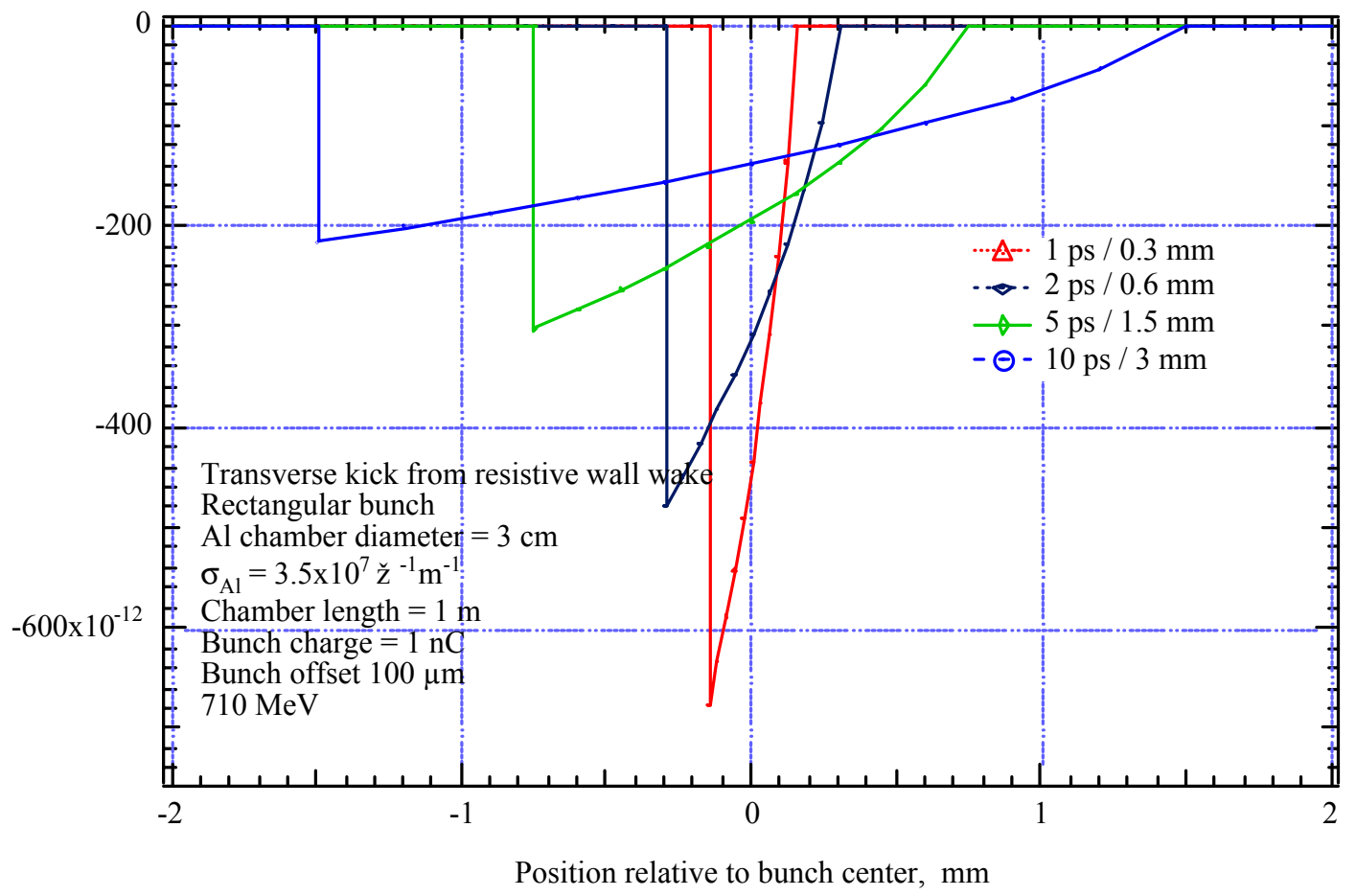

Figure 4-15 Deflection along bunches of 1 - 10 ps length with $1 \mathrm{nC}$ charge, offset $100 \mu \mathrm{m}$, over $1 \mathrm{~m}$ of $3 \mathrm{~cm}$ diameter vacuum chamber at $710 \mathrm{MeV}$, for gaussian (top), and rectangular (bottom) longitudinal distributions. 


\section{Injection (120 MeV)}

To shield from coherent synchrotron radiation effects in the low-energy beam passing through the Arc0 connecting the injector linac to the main linac, the vacuum chamber height is reduced to $5 \mathrm{~cm}$ (in the bending magnet sections only).

For a 3-m beampipe of 5-mm diameter, the distortion along the $2 \mathrm{ps}, 3 \mathrm{nC}$, rectangular bunch with $100 \mu \mathrm{m}$ offset is approximately $3 \%$ of $\sigma_{\mathrm{y}}$. Such an aperture must be aligned to $100 \mu \mathrm{m}$ of the beam orbit.

Since the wake scales as $1 / b^{3}$, an increase in beampipe diameter by a factor of 2 results in a factor 8 decrease in wakefield. A 9-mm diameter beampipe would require alignment to order $0.5 \mathrm{~mm}$ in the $120 \mathrm{MeV}$ dipole magnets to maintain beamsize growth in this section less than $2 \%$.

\section{Photon production arc}

Small-aperture undulators are used in the photon production section, and for a beampipe of $5 \mathrm{~mm}$ diameter at $2.5 \mathrm{GeV}$ with a $2 \mathrm{ps}, 3 \mathrm{nC}$ rectangular bunch we find a distortion of approximately $6 \% \sigma_{\mathrm{y}}$ over $20 \mathrm{~m}$. The vacuum chamber dimensions should be opened up between insertion devices to reduce this effect. Operating with cold walls in the superconducting insertion devices will improve the situation.

\section{COHERENT SYNCHROTRON RADIATION}

Here we present preliminary estimates for a coherent synchrotron radiation (CSR) of electrons in the recirculating linac x-ray source. As described elsewhere [13-15], CSR causes energy loss of electrons and emittance increase in the orbit plane resulting from the energy loss to coherent synchrotron radiation emitted as a bunch passes through the bend magnets. Since the design horizontal emittance is relatively large, small additional emittance growth seems not to be harmful. Therefore, we are mainly concerned with the energy loss and its potentially adverse effect on production of short x-ray pulses through increased energy spread in the bunch.

The electron bunch length and energy varies as a function of location in the recirculating linac lattice, and in each of the several types of dipole magnets. In all cases, we are in the socalled steady-state CSR [15] regime defined as follows:

$$
\varphi_{m} \geq\left(24 \frac{l_{b}}{\rho}\right)^{1 / 3}
$$

where $\varphi_{m}$ is the magnet arc angle, $\rho$ is the bending radius, and $l_{b}$ is the electron bunch length. When the above condition is met the magnet is sufficient long so that radiation emitted by particles in the tail catches and passes the particles at the head of a bunch before the bunch leaves the magnet. The CSR energy loss per unit length of trajectory can then be written as [1315]: 


$$
\frac{d E(s)}{d z} \cong-\frac{2}{3^{1 / 3} \sqrt{2 \pi}} \frac{N e^{2}}{\sigma_{0} \rho^{2 / 3}} \int_{-\infty}^{s}\left(e^{-\frac{s^{2}}{2 \sigma_{0}^{2}}}-e^{-\frac{\left(s^{\prime}-l_{b}\right)^{2}}{2 \sigma_{0}^{2}}}\right) \frac{d s^{\prime}}{\left(s-s^{\prime}\right)^{1 / 3}},
$$

where $N$ is a number of particles per bunch, and $e$ is the electron charge. In deriving Equation (32), we assume a uniform longitudinal density distribution $\lambda(s)=N / l_{b}$ in interval $0<s<l_{b}$ with smooth transitions at the edges with a characteristic length $\sigma_{0}$ as shown in Figure 4-15.

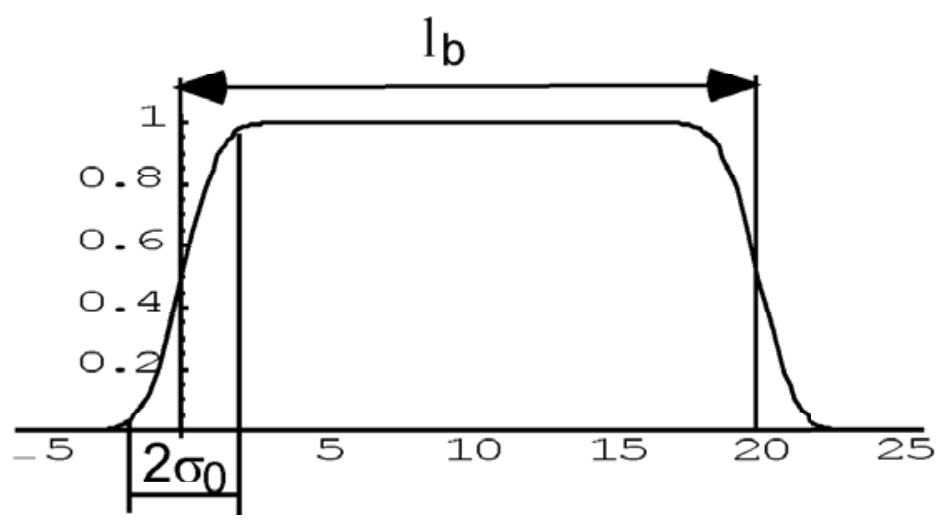

Figure 4-15 Longitudinal density profile of electrons in a bunch.

The integral in Equation (32) can be evaluated using analytical functions and the resulting function $d E(s) / d z$ is shown in Figure 4-16.

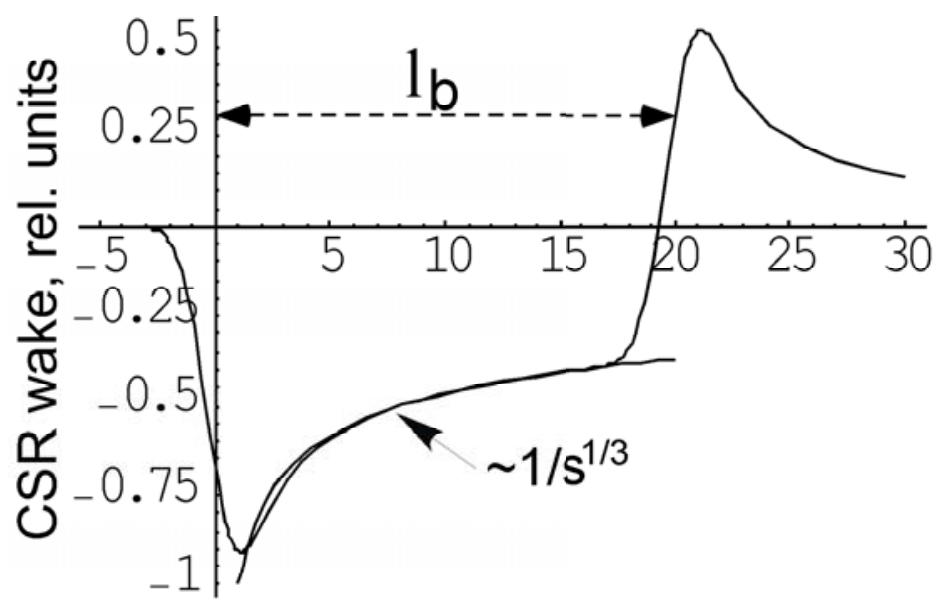

Figure 4-16 CSR wake function $d E(s) / d z$ for an electron longitudinal density distribution shown in Figure 15. 
Note that $d E(s) / d z \sim 1 / s^{1 / 3}$ over the entire length of the bunch excluding the edges. For this functional dependence one can consider partial compensation of the energy variation within the electron bunch induced by CSR, by using off-peak acceleration in the linac.

Using a $1 / s^{1 / 3}$ dependence for the CSR wake function, one can calculate the average energy loss per electron due to the CSR in a magnet of length $L_{m}$ for the main core particles with the expression [15]:

$$
\Delta E=\frac{L_{m}}{l_{b}} \int_{0}^{l_{b}} \frac{d E(s)}{d z} d s \cong \frac{3^{2 / 3} N e^{2}}{\rho^{2 / 3} l_{b}^{4 / 3}} L_{m} .
$$

Table 4-3 lists CSR energy loss for all magnets at all beam energies and bunch lengths. The total energy loss is the so-called a free-space radiation. In practice, the electron bunch travels inside a vacuum chamber that acts as a waveguide for emitted radiation, and does not allow propagation of radiation below a geometry determined cut-off frequency. Thus, not all spectral components of CSR propagate in the vacuum chamber and the actual radiated energy is less than in the free space environment. For an estimate of the shielding effect of the vacuum chamber we follow the analysis suggested in [16]:

$$
\Delta E_{\text {shielded }} / \Delta E_{\text {free space }} \cong 4.2\left(n_{t h} / n_{c}\right)^{5 / 6} \exp \left(-2 n_{t h} / n_{c}\right)\left(n_{t h}>n_{c}\right)
$$

Here $n_{t h}=\sqrt{2 / 3}(\pi \rho / h)^{3 / 2}$ is the threshold harmonic number for propagating waves, $h$ is the height of the vacuum pipe assumed for the purposes of this calculation to be a parallel-plate, and $n_{c}=\rho / \sigma_{c}$ is the characteristic harmonic number for a gaussian longitudinal density distribution with the rms value of $\sigma_{c}$. The meaning of $n_{c}$ is that the spectral component of radiation emitted with harmonic number beyond $n_{c}$ is incoherent. We define $\sigma_{c}=l_{b} / 3.22$, giving a good approximation of spectra for the uniform density distribution compared to the known spectra for a gaussian distribution. Table 4-3 shows two values of vacuum chamber height, $h$ equal to 0.9 $\mathrm{cm}$ and $0.7 \mathrm{~cm}$.

Table 4-3 Coherent synchrotron radiation energy loss in the dipole magnets for free space,

\begin{tabular}{|c|c|c|c|c|c|c|c|c|c|}
\hline Beam energy, MeV & 120 & 120 & 120 & 720 & 1320 & 1920 & 2520 & 2520 & 2520 \\
\hline Bunch length, ps & 20 & 2 & 2 & 2 & 2 & 2 & 2 & 2 & 2 \\
\hline Magnet length, cm & 125.66 & 125.66 & 20.4 & 80 & 80 & 80 & 80 & 20 & 25.4 \\
\hline Magnet field, kG & 3.33 & 3.33 & 6.4 & 11.12 & 10.23 & 9.93 & 10.56 & 9.54 & 20 \\
\hline Energy loss dE, keV & -19 & -409 & -103 & -176 & -111 & -85 & -74 & -17 & -36 \\
\hline \# magnets & 1 & 2 & 2 & 22 & 40 & 58 & 34 & 12 & 6 \\
\hline $\begin{array}{l}\text { Free space energy loss, } \\
\mathrm{keV}\end{array}$ & -19 & -818 & -205 & -3874 & -4447 & -4925 & -2509 & -207 & -215 \\
\hline \multicolumn{10}{|c|}{ Shielded energy loss for vacuum chamber height: } \\
\hline $9 \mathrm{~mm}$ height, $\mathrm{keV}$ & 0 & -419 & -147 & -1209 & -557 & -291 & -47 & -6 & -28 \\
\hline $7 \mathrm{~mm}$ height $\mathrm{keV}$ & 0 & -212 & -98 & -436 & -116 & -40 & 8 & -1 & -6 \\
\hline
\end{tabular}
$9 \mathrm{~mm}$ vacuum chamber, and $7 \mathrm{~mm}$ vacuum chamber as a function of beam energy. 
Appendix A - BBU tracking code

The tracking code is written as a Mathematica notebook. The bunch is divided longitudinally in a number of slices, or macroparticles, that can be choosen by the user. A gaussian or a uniform charge distribution can also be selected.

The linac is modeled as a series of $32 \mathrm{rf}$ cavities, grouped in 4 cryomodules and separated by drift spaces.

The transverse coordinate $x_{i}$ of each slice at the exit of the $i$-th cavity is calculated as a function of the displacement $x_{i-1}$ and angle $x_{i-1}^{\prime}$ at the exit of the preceding cavity:

$$
x_{i}=x_{i-1}+x_{i-1}^{\prime} L_{d r i f t}+x_{i-1}^{\prime} L_{c a v}+\Delta x_{i-1}^{\prime} \frac{L_{c a v}}{2}
$$

The additional transverse kick $\Delta x_{i}^{\prime}$ represents the cumulative effect of the wakefield induced by the upstream macroparticles. The wakefields are calculated using the analytical formula from [3-4].

The macroparticle angle also changes along the linac:

$$
x_{i}^{\prime}=x_{i-1}^{\prime}+\Delta x_{i-1}^{\prime}-\frac{x_{i-1}}{2 L_{c a v}}\left(\frac{\Delta \gamma}{\gamma_{i-1}}\right)^{2}
$$

where the effect of the RF focussing is taken into account by the last term in Equation (A.2).

It is also possible to introduce misalignments between cavities and cryomodules and cryomodules tilts. Again, the user can choose amongst different statistical distribution for these errors and determine the most probable values for the emittance increase, performing simulations using many sets of randomly generated alignment errors.

Finally, the user can arbitrarily select the betatron phase advance between each linac pass.

\section{REFERENCES}

[1] J. Corlett, et al., LBNL-48171 (2001).

[2] A. Chao, B. Richter, C.-Y. Yao, SLAC-PUB-2498 (1980).

[3] A. Mosnier, TESLA 93-11 (1993).

[4] S. De Santis and A. Zholents, LBNL-49483 (2002).

[5] TESLA Technical Design Report (2001).

[6] ALS Alignment Group, private communication.

[7] S. Hartman and J. Rosenzweig, Phys. Rev. E 47, 2031 (1993).

[8] "MERLIN, a C++ Class Library for Charged Particle Accelerator Simulations," http://www.desy.de/ njwalker/MerlinII/index.htm

[9] J. Rosenzweig and L. Serafini, "Transverse Particle Motion in Radio-Frequency Linear Accelerators," Phys. Rev. E, Volume 49 Number 2, February 1994

[10] “MAD Version 8,":http://www-project.slac.stanford.edu/lc/local/AccelPhysics/Codes/Mad /MAD8.html 
[11] M.D.Woodley, private communication

[12] Handbook of Accelerator Physics and Engineering, A. W. Caho and M. Tigner eds, World Scientific, 1998.

[13] L.I. Schiff, Rev. Sci. Instr. 17, 6 (1946).

[14] J.S. Nodvick and D.S. Saxon, Phys. Rev. 96, 180 (1954).

[15] E.L. Saldin, E.A. Schneidmiller, and M.V. Yurkov, NIM A 398, 373 (1997).

[16] R.Li, C.L. Bohn, J.J. Bisognano, Particle Accelerator Conference, 1644 (1997). 


\section{RF PHOTOCATHODE GUN}

\section{OVERVIEW}

The proposed facility requires a high brightness electron beam with an exceptionally low vertical emittance to produce, through beam manipulation and x-ray optics, hard x-ray photon pulses with pulse length of tens of femtoseconds. These requirements are satisfied by a laserexcited, photoemissive electron source, the photoinjector, with the photocathode residing in a high-gradient rf cavity, followed immediately by a short accelerator section to bring the beam up to the $10 \mathrm{MeV}$ range. Generation of low vertical emittance downstream is accomplished by a combination of a finite solenoid magnetic field at the cathode surface and a short skew quadrupole lattice situated downstream from the exit of the rf gun. Table 5-1 lists the parameters required of the flat beam injector.

\section{Table 5-1 Flat Beam Injector Parameters}

\begin{tabular}{|lcl|}
\hline Electron beam parameters at injector exit: & & \\
Energy & 10 & $\mathrm{MeV}$ \\
Charge & $1-3$ & $\mathrm{nC}$ \\
Normalized RMS horizontal emittance & $\sim 20$ & $\square \mathrm{mm}-\mathrm{mrad}$ \\
Normalized RMS vertical emittance @ 1 nC & $<0.4$ & $\square \mathrm{mm}-\mathrm{mrad}$ \\
Energy spread at 10 MeV & \pm 15 & $\mathrm{keV}$ \\
Pulse length (uniform distribution) & 20 & $\mathrm{ps}$ \\
Repetition rate & $\sim 10+$ & $\mathrm{kHz}$ \\
& & \\
RF gun parameters: & & \\
RF frequency & 1.3 & $\mathrm{GHz}$ \\
Peak electric field on a cathode & $\sim 64$ & $\mathrm{MV} / \mathrm{m}$ \\
Normalized geometric emittance at 1 nC & 3 & $\square \mathrm{mm}-\mathrm{mrad}$ \\
& & \\
Laser parameters: & & \\
Wavelength & & \\
(4 ${ }^{\text {th }}$ harmonic of Nd:YLF laser) & 267 & $\mathrm{~nm}$ \\
UV pulse energy at cathode & $\sim 10$ & $\mu \mathrm{J}$ \\
Pulse length (FWHM) & $20+$ & $\mathrm{ps}$ \\
\hline
\end{tabular}

The flat beam photoinjector is composed of two main sections: the rf gun with integrated photocathode, and the flat beam adapter including several skew quadrupoles. A schematic of the injector is shown in Figure 5-1. The $\sim 0.5-\mathrm{m}$ long $\mathrm{rf}$ gun is followed by a short $(\sim 30-40 \mathrm{~cm}) \mathrm{drift}$ section, and then the $\sim 1.5$-m long adapter section. 


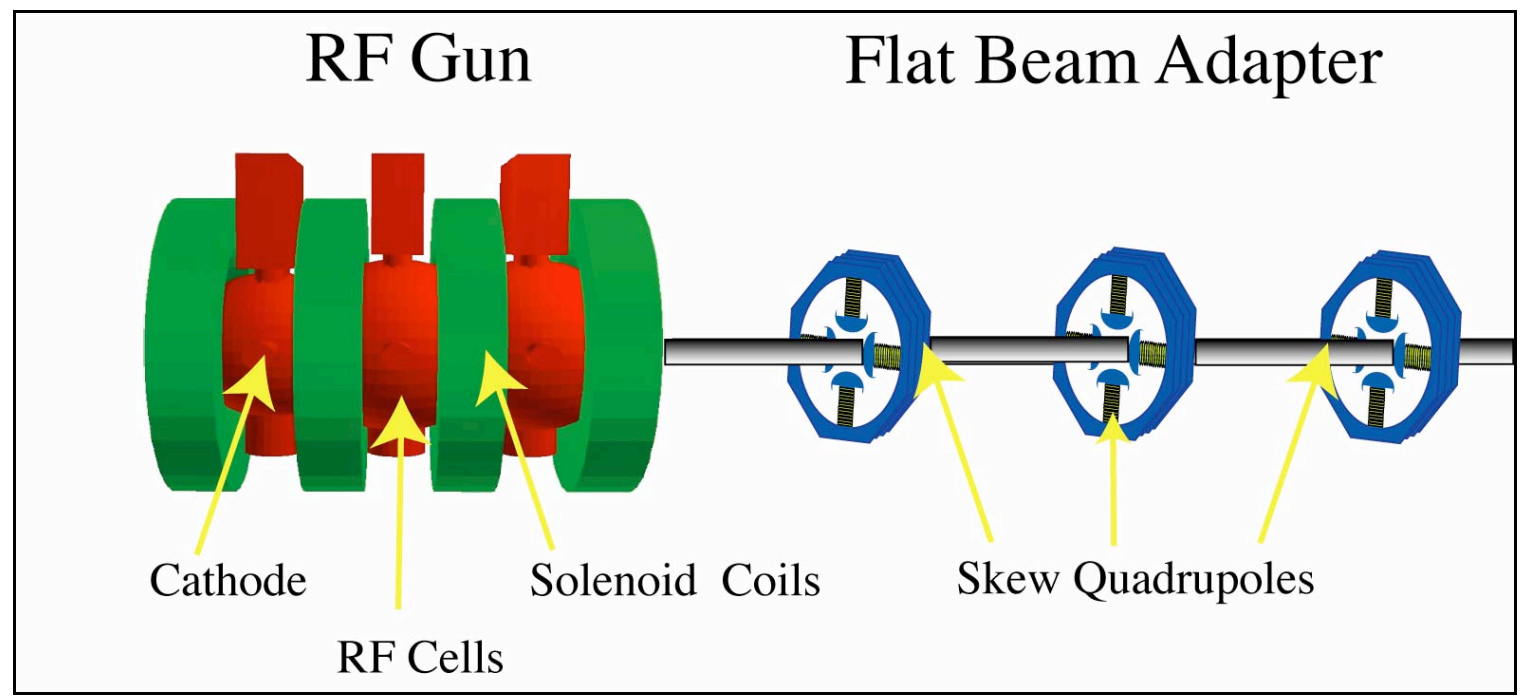

Figure 5-1 Flat beam injector beamline.

\section{Technical Issues for Photoinjectors}

There are difficult technical issues resulting from both the beam requirements tabulated above as well as the operational requirements of high duty factor and low maintenance required of a user facility. We discuss here, issues common to all high brightness photoinjectors, specific issues for flat beam production and transport, and issues relating to high duty factor operation.

\section{Generic issues for high brightness electron beam production}

The electron beam is generated when an intense laser pulse strikes a photocathode. The surface work function of the material defines the necessary laser frequency with which to liberate the electrons. These electrons scatter from the surface to the vacuum region adjacent to the cathode within the first rf cavity. At this point, they are captured by the rf field in the cavity and accelerated. By properly matching the rf cavity phase with the arrival time of the laser pulse, the liberated electrons are captured, accelerated, and passed on to subsequent accelerating cells.

The quantum efficiency (Q.E.) of the photoemissive cathode is the ratio of the number of emitted electrons to the number of incident photons. For various photocathode materials the Q.E varies from $<10^{-4}$ for copper to $\sim 0.10$ for $\mathrm{GaAs}(\mathrm{Cs}, \mathrm{O})$ (see Table 5-2). To produce an electron beam with charge $\mathrm{Q}_{\text {bunch }}$ from a photocathode requires a laser pulse capable of delivering energy 
Table 5-2 Summary of commonly used photocathode materials

\begin{tabular}{||c|c|c|c|c||}
\hline \hline Material & Q.E. Range (\%) & $\square(\mathrm{nm})$ & Lifetime & Vacuum Required (Torr) \\
\hline Metal $(\mathrm{Cu})$ & $0.02-0.06$ & 260 & Months & $10^{-7}$ \\
\hline $\mathrm{CsK}_{2} \mathrm{Sb}$ & $\sim 5$ & 527 & - & $10^{-10}$ \\
\hline $\mathrm{Cs}_{2} \mathrm{Te}$ & $1-5$ & 260 & Months & $10^{-9}$ \\
\hline $\mathrm{LaB}_{6}$ & $\sim 0.1$ & 355 & Months & $10^{-7}$ \\
\hline $\mathrm{GaAs}(\mathrm{Cs}, \mathrm{O})$ & $\sim 10$ & 527 & Days & $10^{-11}$ \\
\hline
\end{tabular}

$$
W_{\text {laser }}=\frac{Q_{\text {bunch }}}{Q . E .} \frac{h c / e}{\square_{\text {laser }}},
$$

where $h c / e$ is $1.24 \mu \mathrm{J}-\mathrm{nm} / \mathrm{nC}$. With a laser wavelength of $0.267 \mu \mathrm{m}(4.64 \mathrm{eV})$ to match the work function of the $\mathrm{Cs}_{2}$ Te surface, this energy is approximately $0.5 \mu \mathrm{J}$ per pulse to produce a $1 \mathrm{nC}$ bunch with a cathode Q.E. of $1 \%$. Operational requirements may push toward production of beams with $3 \mathrm{nC}$ per bunch, or even higher charge. Also, over time, the Q.E. of the photocathode may droop, requiring even larger laser pulse energies to be delivered to the cathode. For these reasons, the laser system is specified to deliver $\sim 10 \mu \mathrm{J}$ pulse.

Laser systems can routinely (even easily) deliver this energy in a single pulse to a photocathode surface. One challenge, however, is to operate these lasers at $10 \mathrm{kHz}$ and above. Thermal loading effects on the optics and amplifier media must be controlled to maintain stability and beam quality.

From the standpoint of beam dynamics and user operations, the laser must display short-term and long-term stability in its operation. Of greatest concern is the spatial-temporal distribution of the laser pulse at the cathode surface. The laser must deliver an illumination onto the cathode that is uniformly distributed in the transverse plane, as well as uniform in time along the pulse. Any deviations will induce non-uniformities in the electron beam distribution that can drive emittance growth downstream. Laser pulse shaping techniques in both the transverse and longitudinal dimensions are currently under development [1,2].

In addition to the laser, the photocathode must display uniform emission under optimal (uniform) illumination. The local distribution of quantum efficiency on the cathode surface must be uniform to produce a uniform beam. Thermal emittance characteristics of photocathode materials depend on the laser wavelength, angle of incidence, surface treatment and vacuum, and surface temperature. At present, understanding of the limiting effects on thermal emittance is only at a rudimentary level. There has been some theoretical work, but almost no experimental measurements. Work is underway at various laboratories $[3,4]$ to quantify the thermal emittance characteristics of various photocathode materials.

The vacuum requirements on coated photocathodes are severe, lest they display significant degradation in their quantum efficiency. They are typically prepared by sputtering onto a molybdenum substrate, which can then be stored for months in ultra-high vacuum $\left(\sim 10^{-11}\right.$ torr $)$ with little degradation of the surface work function and quantum efficiency. For optimal 
performance, cesium-telluride photocathodes must be operated in a vacuum of $\sim 10^{-9}$ torr. Operationally, cesium-telluride cathodes are known to degrade over a period of several months, with the quantum efficiency dropping from $\sim 5-10 \%$ to $\sim 0.5-1 \%$. The initial quantum efficiency can often be recovered by UV treatment of the surface [5].

The use of $\mathrm{Cs}_{2} \mathrm{Te}$ photocathodes in rf guns is relatively mature, and provides a useful set of parameters to create a working point design for the injector and ancillary drive laser system. It is not, however, the only available material. We will continue study into the use of alternative photocathode materials in order to ascertain the best choice, given our operating conditions of high duty factor, high brightness, and (relatively) low maintenance.

In a generic rf photoinjector the electron beam emittance is minimized by fast acceleration of the bunch to kinetic energies on the order of a few $\mathrm{MeV}$. At this point, the beam may be transported for a short distance ( tens of $\mathrm{cm}$ to $\sim 1 \mathrm{~m}$ ) before suffering from excessive space charge induced blow up. External solenoid focusing is used to balance the space charge forces so that the beam is prepared for injection into the subsequent accelerator section. During acceleration, the influence of space charge on emittance blow-up rapidly diminishes as the beam energy increases. The emittance at the entrance to the injector linac is essentially Frozen in.

High brightness photoinjectors are characterized by their normalized peak brightness,

$$
B_{\text {peak }}=\frac{I_{\text {peak }}}{v_{4}}=\frac{2 I_{\text {peak }}}{\square_{1}, \square_{\text {, }}}
$$

where $I_{\text {peak }}$ is the peak current carried by the beam, and the $4 \mathrm{D}$ rms transverse phase-space volume is characterized by the normalized rms mittances $\left(\square\right.$, and $\square_{\text {, }}$ ) via $\square_{4}=\square_{k}, / 2$. Figure 5-2 shows some recently constructed and commissioned photoinjectors, and the relation between peak brightness and operating $\mathrm{rf}$ frequency. The proposed flat beam photoinjector is added to the plot for comparison, and is seen to perform similarly to both the FNPL and TTF photoinjectors. 


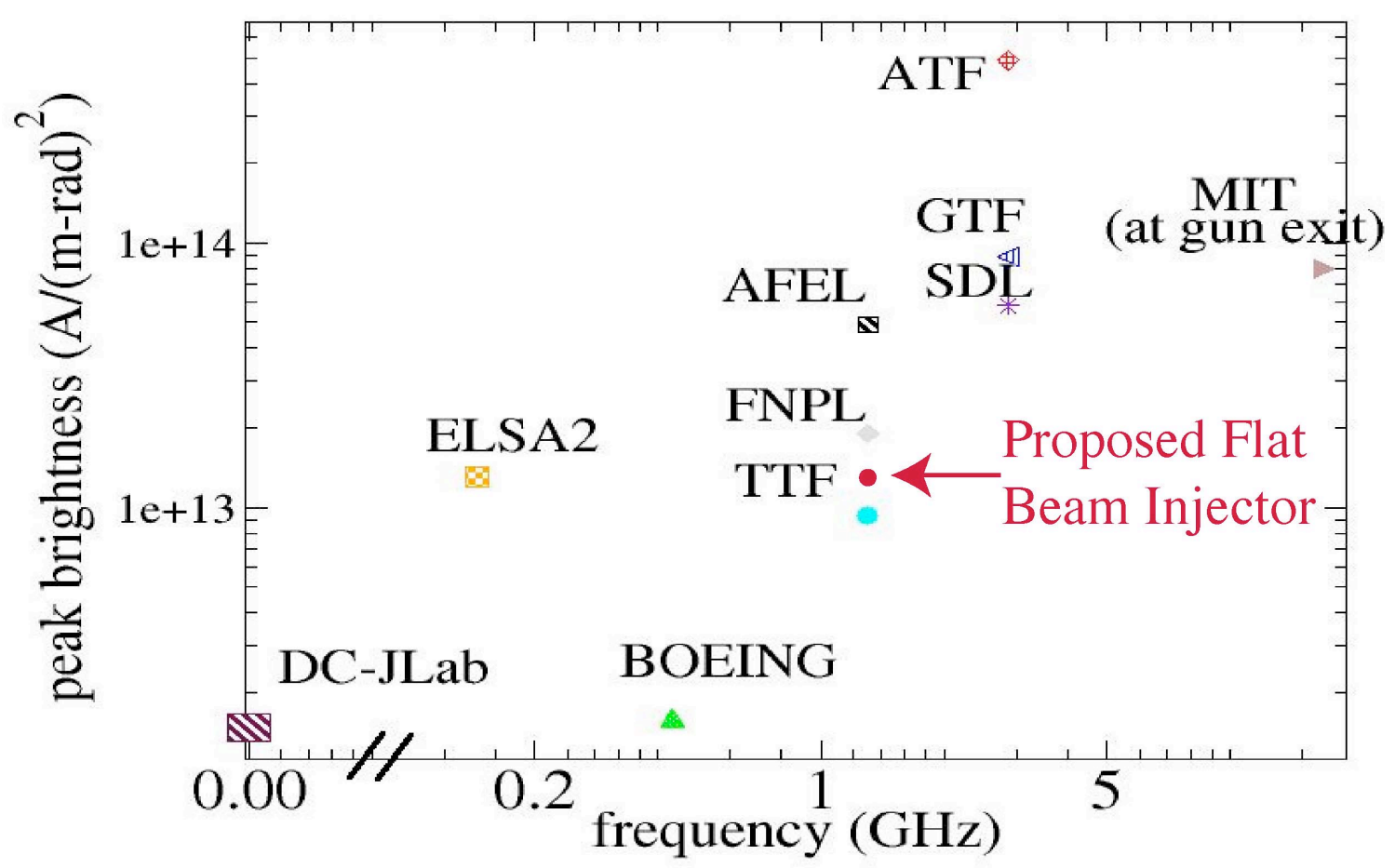

Figure 5-2 Measured peak brightness of recent photoinjectors. The proposed flat beam injector design brightness has been included for comparison. (Figure courtesy of P. Piot).

Specific issues for flat beam production

The flat beam photoinjector and transport scheme differs in some very important ways from the generic photoinjector described above. While this design shares the same issues as the generic case, it also brings in several new, specific issues.

In contrast to generic photoinjectors that employ reversed-polarity solenoids (bucking coils) to negate any residual magnetic field at the cathode plane, the generation of a flat beam through electron optics begins with the imposition of a strong solenoidal magnetic field at the photocathode. This will produce the necessary correlation between the horizontal and vertical phase spaces that ultimately results in a large emittance ratio. However, the presence of a large magnetic field at the cathode significantly changes the beam optics through the rf gun and beyond.

Additionally, upon exit from the gun the beam must be carefully matched to the flat beam adapter beamline. This short beamline of skew quadrupoles transforms the correlated, 4-D transverse phase space of the beam into uncorrelated, 2-D sub-spaces with a large horizontal: vertical emittance ratio. To do so, the quadrupole lattice must produce specifically tailored betatron phase advances in the horizontal and vertical planes.

The flat beam must be transported with a conventional quadrupole lattice and matched into the bunch compressor and subsequent linac structure. Localized variations in the bunch density, 
transverse and longitudinal profiles, and bunch-to-bunch charge fluctuations must be carefully handled to minimize emittance growth at all stages, particularly up to the entrance of the first linac.

\section{Thermal management in the high duty factor rf gun and laser}

The high repetition rate, $10 \mathrm{kHz}$ or more, of the 20 -psec beam pulse represents a low beam duty factor. However, the relatively long filling time of the cavity results in a $5 \%$ rf duty factor, with a correspondingly high wall power density. Careful thermal management of the photoinjector is necessary. The laser system must also be carefully designed to operate at such high duty factors, as previously mentioned.

This chapter and the following three chapters discuss the current design photoinjector and address our understanding of the issues involved in producing flat beams of large emittance ratio. The first section presents our current design for the rf gun, including calculations of electromagnetic mode structure and mechanical stress analysis from inductive heat loading, $\mathrm{rf}$ drive requirements, and the placement of focusing solenoids. The second section discusses beam dynamics within the rf gun, including space charge effects, the role of beam launch timing with respect to the $\mathrm{rf}$ phase, and the effect of offset phasing between the different rf gun cells. The third section examines the process of emittance compensation for angular momentum dominated beams. Theoretical tools to help understand compensation are discussed, followed by recent experimental results. The last section describes previous and current studies on the flat beam adapter optics. The basic process is described in detail followed by results from a proof-ofconcept experiment. Finally, a preliminary design for the flat beam adapter to be used in the proposed injector is presented, with the results from simulation that demonstrate the required low vertical emittance.

\section{RF Gun EleCtromagnetic DeSign}

The photoinjector will comprise a high-gradient reentrant gun cell with a $\mathrm{Cs}_{2} \mathrm{Te}$ photocathode producing a $20 \mathrm{psec}$ bunch with a peak current in the 50 ampere range, followed immediately by a 3-cell $\pi$-mode acceleration structure accelerating the beam to $10 \mathrm{MeV}$. All the cavities will be independently driven and phased. Calculations are presented on the design and performance of the rf gun cavities using the electromagnetic codes SUPERFISH [6], MAFIA [7], and NSYS [8].

Figure 5-3 shows the overall layout of the four cells, the left-most cell containing the photocathode, generating and accelerating the $20 \mathrm{psec}$ bunch to $2 \mathrm{MeV}$, followed by a 3-cell $\pi$ - mode accelerating structure, bringing the beam energy to $10 \mathrm{MeV}$. 


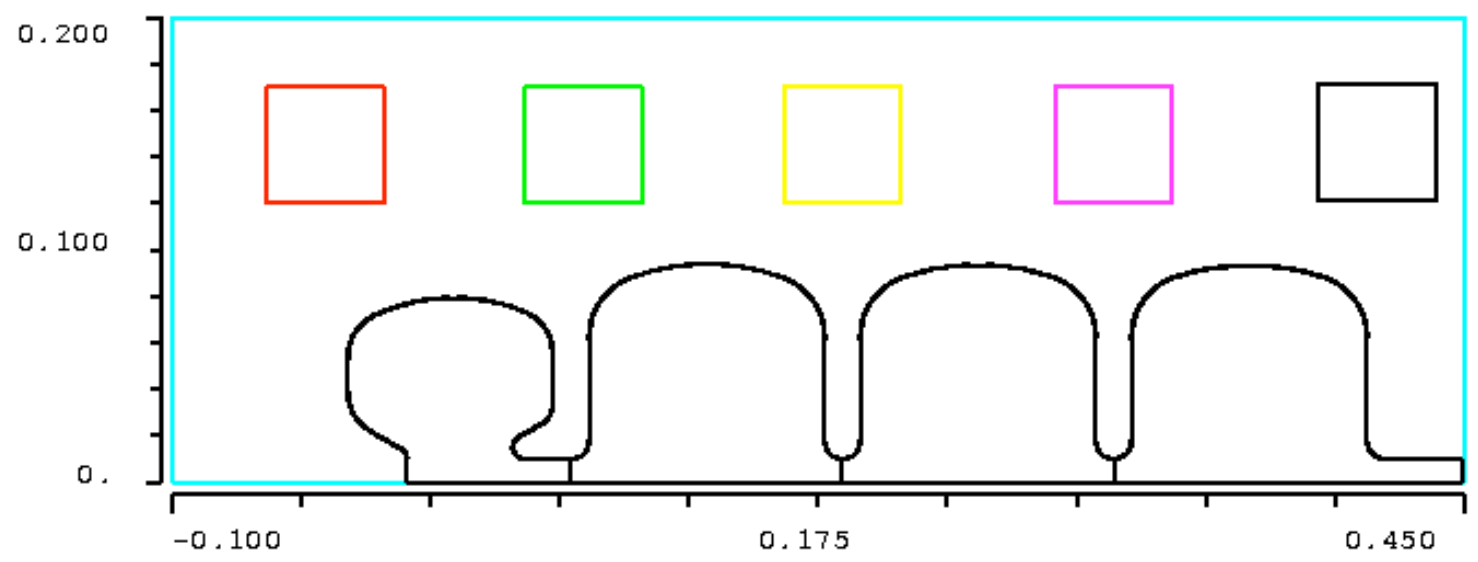

Figure 5-3 2-D model of rf gun cavities and solenoid magnets (MAFIA).

Solenoids surround the entire 4-cell structure, with waveguide couplers interposed between the separate windings. The longitudinal magnetic field at the cathode will be adjustable, to prepare the proper angular momentum of the launched beam for the flat beam transformer

The $1.3 \mathrm{GHz}$ operating frequency is chosen as a compromise between surface power density and achievable electric field gradient. The $10 \mathrm{kHz}$ repetition rate, folded with a 1/e cavity filling time of 3.4 microseconds with a loaded $Q_{L}$ of approximately $10^{4}$, requires that the cavity be overdriven to achieve full gradient in 5 microseconds. The cavity field variation during the 20-psecbeam pulse will be negligible, though pulse-to-pulse reproducibility is very important for timing stability.

\section{Cell 1}

The first cell is shaped to maximize both the beam energy at the end of the first cell and the field at the photocathode, while minimizing the peak electric field elsewhere in the cavity, minimizing the dark current, and reducing the tendency toward sparking. To minimize multipactoring and conditioning time, the walls of the cavity comprise sections of arcs, rather than parallel straight wall sections, used in former pillbox photoinjector configurations. 


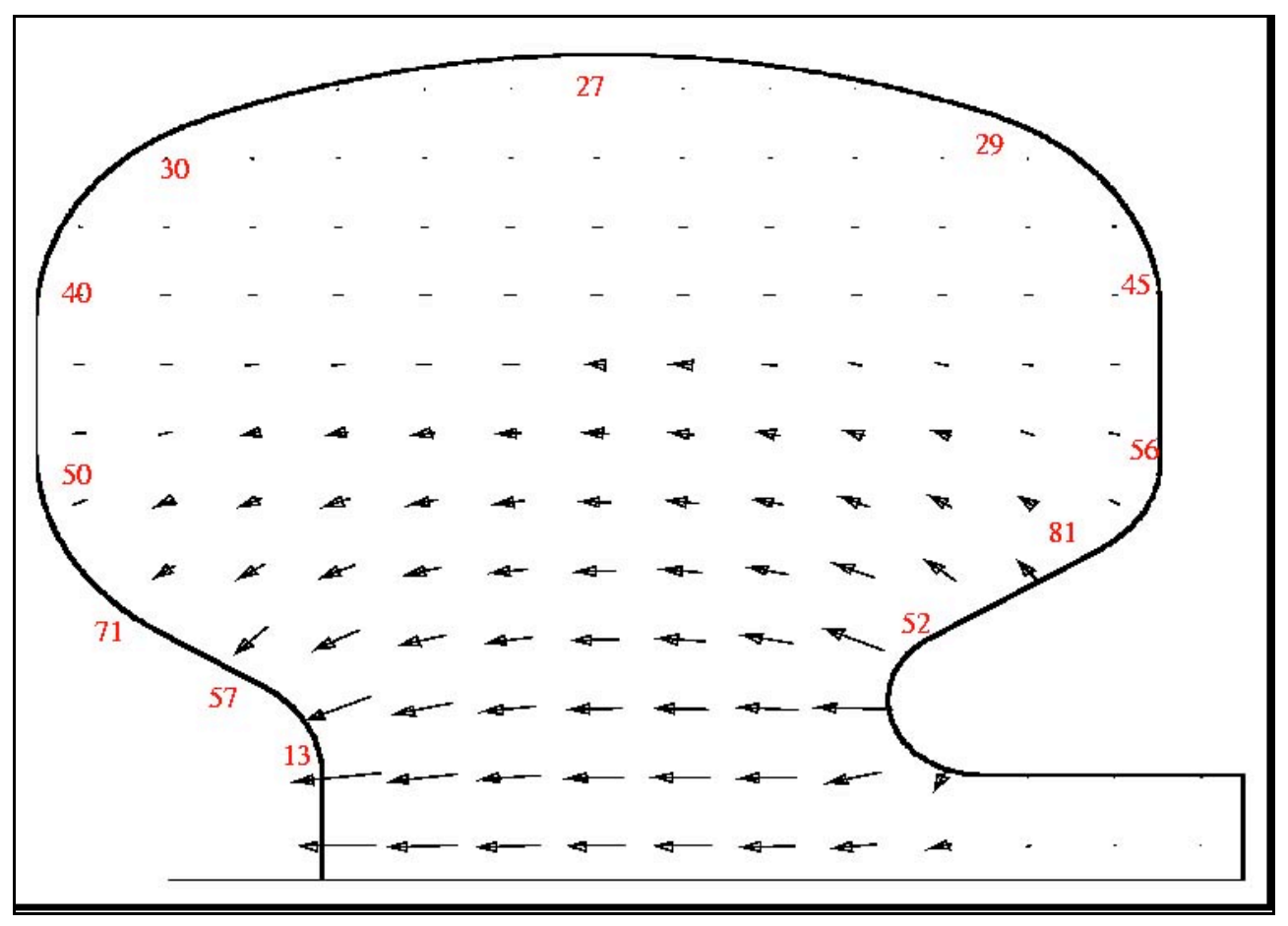

Figure 5-4 Cell-1 2-D geometry and accelerating mode electric field (SUPERFISH). The red numbers denote the corresponding average heat flux $\left(W / \mathrm{cm}^{2}\right)$ into the cavity at significant points.

Figure 5-4 shows the cavity outline, with the photocathode sited on a mesa, facing a reentrant nose-cone that increases the local field on the cathode surface and increases the shunt impedance of the cavity itself. The figure also shows the electric field lines for the $1.3 \mathrm{GHz}$ fundamental mode. To achieve $64 \mathrm{MV} / \mathrm{m}$ electric field at the photocathode surface, $752 \mathrm{~kW}$ peak rf power is required. Beam loading is insignificant for a $1 \mathrm{nC}, 20$ psec beam pulse accelerated to $2 \mathrm{MeV}$. The extracted beam energy is $\sim 2$ millijoule where the stored energy in the cavity is 1.95 joule at operating gradient. Table 5-3 lists cathode cell parameters.

The peak azimuthal $\mathrm{H}$-field over the rf pulse produces an RMS power dissipation in $100 \%$ steady-state operation of 2300 watts $/ \mathrm{cm}^{2}$, which, when operated at the drive power time dependence described below, becomes an RMS power dissipation of $81 \mathrm{watts} / \mathrm{cm}^{2}$ in the upper part of the exit nosecone and much less elsewhere in the cavity. Integrated over the entire cavity, the average RMS thermal power dissipation is $38 \mathrm{watts} / \mathrm{cm}^{2}$ at the peak of the rf pulse.

The long exit beampipe reduces the coupling between the first cell and the following $\square$-mode accelerating structure to essentially zero. The phasing between the first cell and the acceleration section is adjustable. 
Table 5-3 Cathode cell characteristics

\begin{tabular}{|lll|}
\hline Frequency & 1.30 & $\mathrm{GHz}$ \\
$\mathrm{Q}_{0}$ & 22000 & \\
Peak Power & 752 & $\mathrm{~kW}$ \\
Peak E $\mathrm{c}_{\text {cathode }}$ & 64 & $\mathrm{MV} / \mathrm{m}$ \\
Peak $\mathrm{E}_{\text {wall }}$ & 87 & $\mathrm{MV} / \mathrm{m}$ \\
Peak wall magnetic field & 71 & $\mathrm{kA} / \mathrm{m}$ \\
Transit time factor $(\mathrm{T}), \mathrm{\square}=1$ & 0.821 & \\
$\mathrm{Z}_{\text {shunt }}$ & 7.19 & $\mathrm{Mohm}$ \\
$\mathrm{Z}_{\text {shunt }} \mathrm{T}^{2}$ & 4.84 & Mohm \\
\hline
\end{tabular}

\section{The 3-cell Acceleration Section}

The cathode cell is followed by a 3-cell accelerating structure of conventional design. Each $1.3 \mathrm{GHz}$ cavity $(11.54 \mathrm{~cm}$ between cavity centers) supports a peak axial E-field of $40 \mathrm{MV} / \mathrm{m}$ to accelerate the electron bunch to a final energy of $10.2 \mathrm{MeV}$.

The coupling between the three accelerating cells is small, and each cell is independently driven through two waveguide ports, to reduce the peak power density at each drive port, and to eliminate any transverse dipole moment.

\section{Cavities - RF Drive}

The four cavities in the photoinjector will be driven independently. Each cavity will be driven from a pair of slot waveguide couplers to eliminate any tendency of exciting transverse dipole modes in the cavities, and to reduce the incident power density on the rf windows and coupling apertures. Figure 5-5 shows a MAFIA mesh of the first cell with the waveguide-driven slot coupler, in quarter-cut symmetry

Table 5-4 lists a summary of the characteristics of each of the three identical accelerating cells.

Table 5-4 Characteristics of rf gun accelerating cells

\begin{tabular}{|lll|}
\hline Frequency & 1.30 & $\mathrm{GHz}$ \\
$\mathrm{Q}_{0}$ & 29000 & \\
Peak rms Power & 1334 & $\mathrm{~kW}$ \\
Peak $\mathrm{E}_{\text {axis }}$ & 40 & $\mathrm{MV} / \mathrm{m}$ \\
Peak $\mathrm{E}_{\text {wall }}$ & 49.4 & $\mathrm{MV} / \mathrm{m}$ \\
Transit time factor $(\mathrm{T}), \mathrm{\square}=1$ & .689 & \\
$\mathrm{Z}_{\text {sh }}$, each cell & 6.09 & $\mathrm{Mohm}$ \\
$\mathrm{Z}_{\text {sh }} \mathrm{T}^{2}$, each cell & 2.89 & $\mathrm{Mohm}$ \\
Energy gain/cavity & 2.77 & $\mathrm{MeV}$ \\
\hline
\end{tabular}




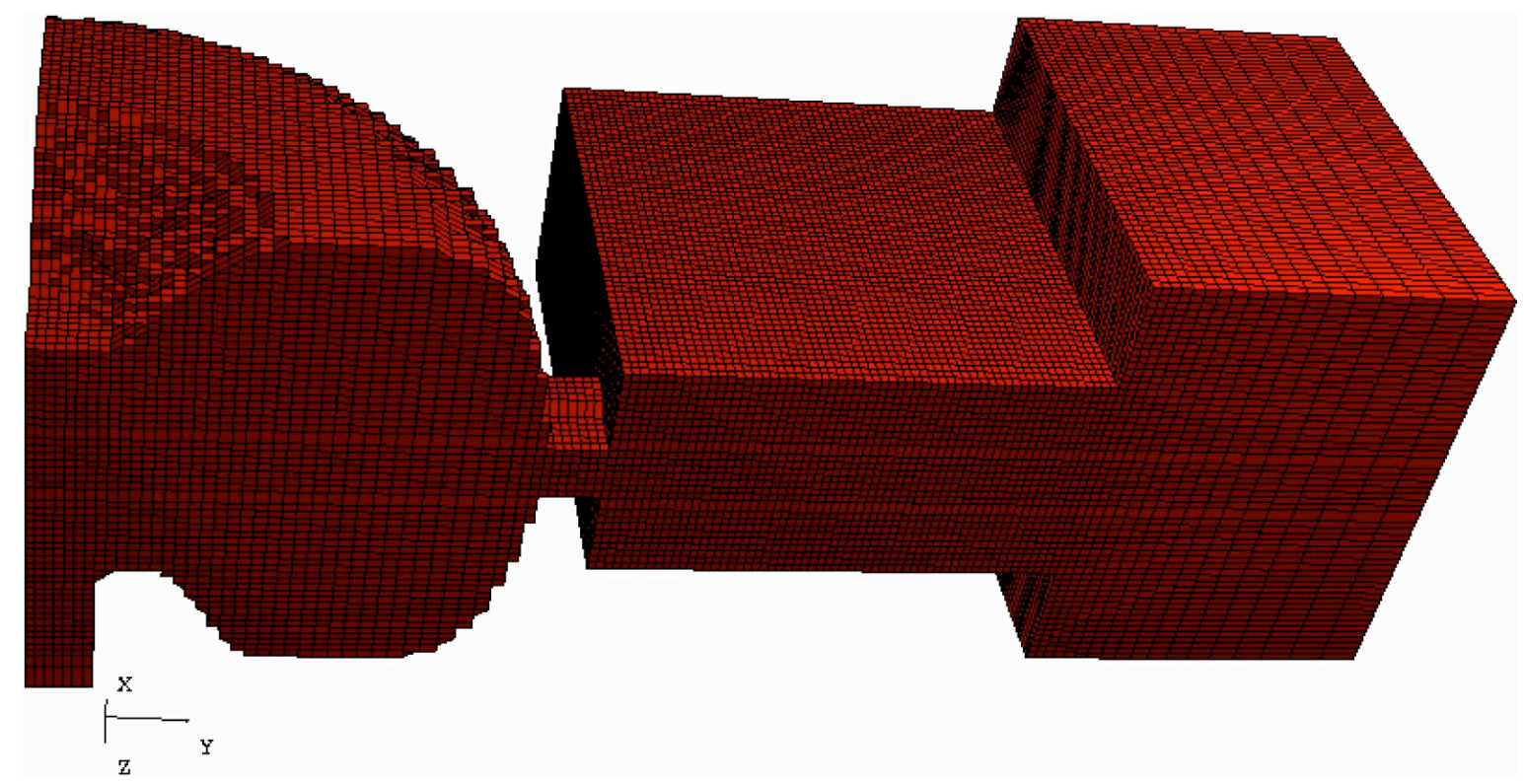

Figure 5-5 3-D model of rf cell, coupler, and input waveguide (MAFIA).

A full-height waveguide feeds a quarter-wavelength of half-height waveguide, which fits in between the solenoids, and which in turn drives the coupling slot itself. Each of the two coupling slots, located on the equator of the cavities, has a cross-sectional area of approximately $4 \mathrm{~cm}^{2}$, giving critical coupling, and is $1 \mathrm{~cm}$ long in order to provide space for the water cooling passages incorporated into the wall.

The cross-sectional profile of the coupling slot is a dumbbell shape, with the radii of the ends of the profile $0.75 \mathrm{~cm}$, and with the centers of the two ends separated by $2.5 \mathrm{~cm}$. This profile will minimize peak magnetic fields and therefore peak surface power dissipation that usually occurs at the ends of slot couplers.

Each of the four cells will be independently driven from separate klystrons, through circulators and power dividers into each of the two drive ports. The phasing of the rf drive to each of the three accelerating cavities is adjusted for $\pi$-mode operation. The small iris diameter $(2 \mathrm{~cm})$ effectively decouples the cavities from each other. The impact of transverse dipole wakefield from these irises upon emittance growth and energy spread is assessed.

The phasing between the first cell and the three acceleration cells is variable, and due to the long exit snout of the first cell, the coupling to the accelerating cells is effectively zero. In addition, the phasing of the laser pulse to the photocathode is also independently adjustable, with the electron emission occurring at a rather early $\left(20^{\circ}\right)$ phase relative to the rf phase in the first cell. The bunch passes through the centers of the three accelerating cells on the rf crest. 


\section{Cavity Wall Power Density and RF Drive Waveform}

The wall power density is of critical interest in this high duty factor injector. Nominally, the repetition rate is $10 \mathrm{kHz}$ with an $\mathrm{rf}$ pulse length of 5 microseconds, for a macro duty factor of $5 \%$. This is much higher than any existing photoinjector and results in a high average power to the cavities.

The pulse length of 5 microseconds is only $50 \%$ greater than the $1 / e$ cavity filling time of 3.4 microseconds. If the accelerating cavity is driven at its asymptotic power of $1.33 \mathrm{MW}$, the gradient will have risen to only $77 \%$ of full gradient at 5 microseconds. To bring the cavity to full gradient in 1.5 filling times, the drive voltage must be increased by $30 \%$, corresponding to a drive power increase of $69 \%$, or, for the accelerating cavities, about $2.5 \mathrm{MW}$.

The envelope of the rf waveform in the cavities will not be a square wave, but will clearly show the exponential rise and fall during and after the pulse. This waveform will actually reduce the average thermal power in the cavities.

If the cavities are overpowered by $69 \%$ for 5 microseconds and then allowed to decay after the 20 psec beam pulse, the actual thermal load is only $80 \%$ of an equivalent thermal load of a 5 microsecond square waveform in the cavities.

The average thermal power can be further reduced by actively removing the power from the cavities by reversing the drive phase of the applied power, which will remove the stored energy over a 2 microsecond period and deposit it in the dummy load associated with the circulator.

If the stored energy is actively removed from the cavity as described above, the total thermal power is reduced to only $59 \%$ of the equivalent square wave rf pulse in the cavities. Under the assumption that the actual cavity $Q_{0}$ is $85 \%$ of the theoretical copper $Q_{0}$, the wall power density savings are significant, with the worst-case wall power density in the first cell decreasing from 116 watts $/ \mathrm{cm}^{2}$ for the square-wave case to 81 watts $/ \mathrm{cm}^{2}$ with active stored energy removal and $85 \%$ copper conductivity.

The upper plot of Figure 5-6 shows the cavity voltage waveform for a 5 microsecond pulse and a 3.4 microsecond filling time with unity coupling, overdriven to achieve $100 \%$ gradient at 5 microseconds. The lower plot shows the effect of reversing the drive phase at 5 microseconds and continuing the drive for another two microseconds. In this case, the integrated thermal power is only $59 \%$ of the square-wave power. Studies show that increasing the coupling, reducing $\mathrm{Q}_{\mathrm{L}}$ and the filling time do not significantly lower the average thermal load in the cavity.

The rf gun will require $8.7 \mathrm{MW}$ of peak power, with 1.2 MW for the first cavity, and $2.5 \mathrm{MW}$ for each of the three accelerating cavities. At 5\% duty factor, 5 microsecond pulse length at $10 \mathrm{kHz}$, the average power requirement will be $435 \mathrm{~kW}$, or possibly more, if the phase flip technique of actively removing the stored energy from the cavity is used, where the pulse length may extend to 7 microseconds.

To obtain maximum flexibility for the adjustment of the flat beam parameters, the preferred solution is an arrangement of four independent klystron systems, one for each cavity in the photoinjector. This allows one to take full advantage of fast individual control of each resonator by a digital controller acting directly on a low-power vector modulator, avoiding the use of a high-power rf distribution network with ferrite phase shifters and attenuators. 

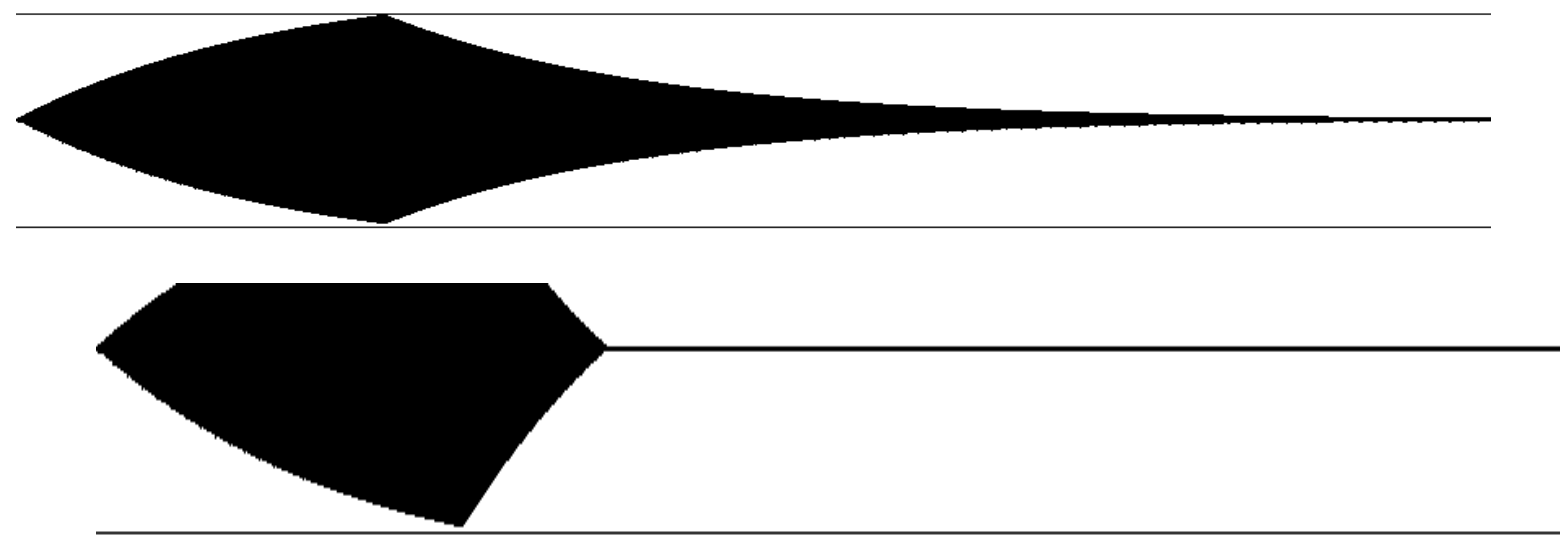

Figure 5-6 Cavity gradient with 5 microsecond rf pulse, $3.4 \mu$ s fill time. Top - no active stored energy removal; bottom - active stored energy removal

\section{Mechanical Considerations}

The four cavities that comprise the photoinjector will operate at a nominal $5 \%$ duty factor, or perhaps even higher. The wall power density will approach $81 \mathrm{watts} / \mathrm{cm}^{2}$ in certain regions of the OFHC cavity, and will average 38 watts $/ \mathrm{cm}^{2}$ over the entire cavity, which will require careful thermal management, but is within the conservative operating range for room temperature, water-cooled copper structures.

The photoinjector will incorporate water-cooling passages integral to the cavities themselves. ANSYS modeling of the temperature and mechanical stress starts with determining the wall power density with the $e$-mag electromagnetics module, cross-checked with MAFIA simulations to ensure that the wall power density in the vicinity of the drive ports is modeled correctly. Figure 5-7 shows an ANSYS model of an earlier configuration of a 4-cell photoinjector with large drive ports, now replaced with slot couplers, and an initial configuration of integral watercooling passages.

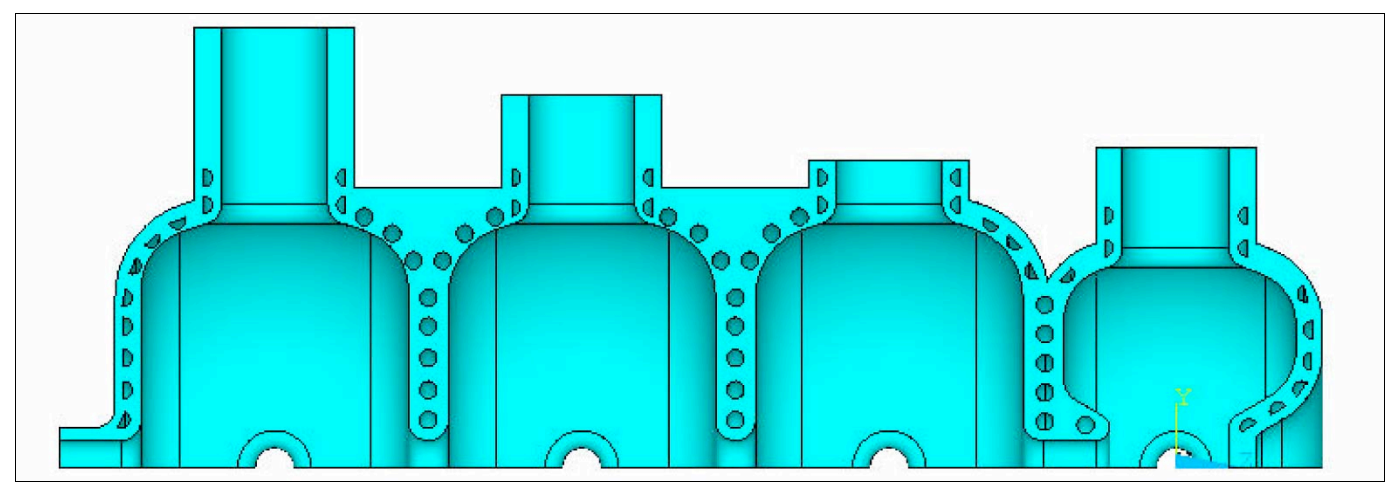

Figure 5-7 ANSYS cavity geometry. 
An ANSYS model of the 4-cell cavity has been assembled, and preliminary calculations have been carried out to investigate the temperature rise. Mechanical stress within the cavity structure will be studied in the future.

Figure 5-8 shows the temperature rise with $33 \mathrm{~kW}$ dissipated in each cavity. This is $71 \%$ of the power that would be dissipated with the active stored energy removal scheme and therefore would show $71 \%$ of the expected temperature rise. Subsequent calculations will reflect the revised rf drive geometry and will be renormalized to the expected wall power density.

The photocathode plug will be removable and will be introduced from the photocathode preparation chamber. The high electric fields and high displacement currents in this region will require careful engineering of the cathode assembly to integrate well into the cavity structure, and be compatible with high vacuum requirements and surface preparation methods. In addition, the geometry immediately surrounding the cathode may be modified to produce a small radial focusing field in the first cell.

During rf conditioning of the electron gun, the cathode will be replaced with an un-coated molybdenum plug, so as to avoid contamination or degradation of the surface coating due to vacuum breakdown events. After the gun has been conditioned, the molybdenum plug will be replaced with a fully coated cesium-telluride cathode.

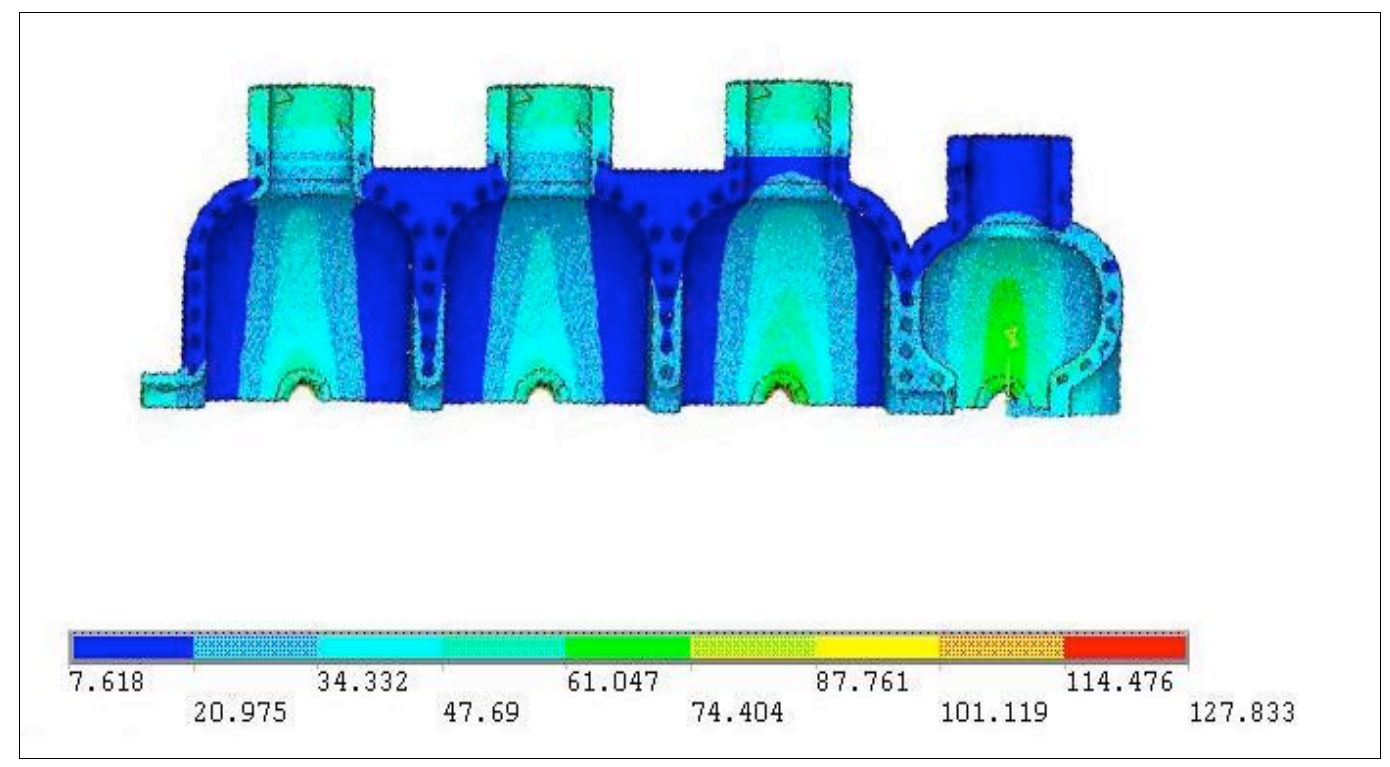

Figure 5-8 ANSYS model of steady-state thermal loading in the rf gun.

\section{Higher Repetition Rates}


The total rf power demand $(752+3 \times 1330=4742 \mathrm{~kW})$ is large, and at $5 \%$ duty factor at a $10 \mathrm{kHz}$ pulse rate approaches a quarter of a megawatt average power needing to be conducted away from the cavity. Operation at a higher pulse rate would require essentially $\mathrm{CW}$ operation of the cavities. In this case, the peak fields in the cavities would be reduced and the beam dynamics adjusted accordingly.

\section{FOCUSING SOLENOIDS}

The beam will be launched from the cathode immersed in a longitudinal magnetic field, and be focused throughout the 4-cell structure by three or four more solenoids through to the exit. The magnetic field strength on axis at the cathode is adjustable over the 1-1.4 kgauss range to prepare the beam for the flat beam transformer, and to allow for emittance compensation.

Four or five solenoid coils surround the structure, each with a cross section of $5 \mathrm{~cm}$ by $5 \mathrm{~cm}$, with a major radius of $13.5 \mathrm{~cm}$. The $\mathrm{rf}$ waveguide feeds and other cavity utilities occupy the space between the solenoids. Each solenoid will operate up to an excitation of 20000 ampereturns, or a current density of about $5100 \mathrm{amps} / \mathrm{in}^{2}$. With at least a $70 \%$ packing factor, this represents a reasonable margin of safety. Figure 5-9 shows five solenoids surrounding a 4-cell structure.

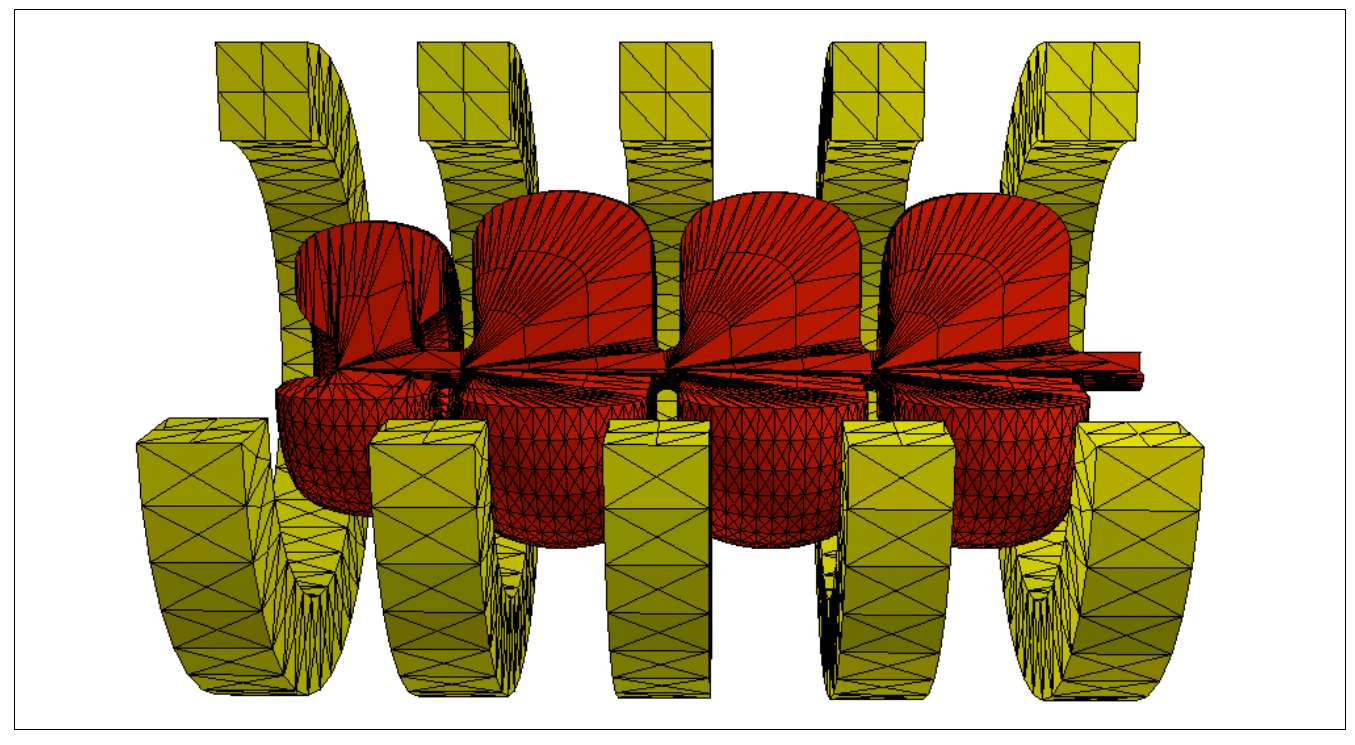

Figure 5-9 MAFIA 3-D model of 4-cell rf gun cavities and solenoid coils. The photocathode is located at the far left.

\section{REFERENCES}


[1] J. Yang, et al., 'Experimental Studies of Photocathode RF Gun with Laser Pulse Shaping', to appear in the Proceedings of the Eighth European Particle Accelerator Conference (EPAC 2002), Paris, France, 2002.

[2] H. Tomizawa, et al., 'Reduction of electron-Beam Emittance with Shaping Both Spatial and Temporal Profiles of UV Laser Light source for Photo-Cathode RF Gun', to appear in the Proceedings of the Eighth European Particle Accelerator Conference (EPAC 2002), Paris, France, 2002.

[3] P. Michelato, et al., 'Thermal Emittance Estimation Using a Time-of-Flight Spectrometer', Proceedings of the Seventh European Particle Accelerator Conference (EPAC 2000), Vienna, Austria, 2000.

[4] B. M. Dunham, et al., 'Emittance Measurements for the Illinois/CEBAF Polarized Electron Gun', Proceedings of the 1995 Particle Accelerator Conference, (IEEE, New York).

[5] W. Hartung, et al., 'Studies of Photo-Emission and Field Emission in an rf Photo-Injector with a High Quantum Efficiency Photo-Cathode', Proceeding of the 2001 Particle Accelerator Conference, (IEEE, New York), 2001.

[6] K. Halbach and R. Holsinger, Particle Accelerators, 3, 213 (1976).

[7] T. Weiland, et al., Proceedings of the Linear Accelerator Conference, SLAC-303, 282 (1986).

[8] ANSYS is a registered trademark of ANSYS, Inc. (www.ansys.com) 


\section{BEAM DYNAMICS IN THE RF GUN}

We have used three distinct computer simulation codes to model the behavior of the beam in the rf gun and the following beamline. HOMDYN [1] models the beam as a sequence of slices, with each slice propagated by a set of envelope equations. This code executes rapidly and may be used to model very long stretches of beamline. We use HOMDYN for initial parameter scans, to identify possible operating points. PARMELA [2] tracks particles in external fields via integration of the Maxwell-Lorentz equations of motion. Self-field effects are included in the electrostatic approximation, calculated in the beam's center-of-momentum frame. PARMELA has been used extensively to examine emittance growth in the rf gun and to further explore the possible operating points. MAFIA is a self-consistent electromagnetic solver that may track particles using a 2D relativistic particle-in-cell (PIC) module (TS2400). MAFIA simulations are intensive both in CPU cycles as well as in memory storage requirements. Hence, simulations with MAFIA are limited to exploring the detailed beam dynamics within the rf gun only, as a means of calibrating and benchmarking simulations with HOMDYN and PARMELA.

\section{Cross-Code Benchmarking Tests}

We have produced simulations and comparisons between the codes by modeling the previously considered 3-cell rf gun [3]. The gun was simulated in each of the codes for a range of operating parameters. For simplicity, the beam is assumed to have rectangular profiles both in radius and in time, with uniform charge density. The spot size on the cathode has a radius of $2 \mathrm{~mm}$, which represents a reasonable working point to balance initial thermal emittance with transverse space charge. For these initial tests to benchmark the codes, the thermal emittance was set to zero. The design goal is a geometric-averaged normalized emittance of $3 \mathrm{~mm}$-mrad at the output of the gun. The bunch parameters are recorded after the first cell $(\mathrm{z}=65 \mathrm{~mm})$, and at the exit of the gun $(z=300 \mathrm{~mm})$. In these simulations, a 3-cell model was used. The peak (on-axis) electric field in the first cell was $64 \mathrm{MV} / \mathrm{m}$, while the second and third cells had peak fields of $43 \mathrm{MV} / \mathrm{m}$ each.

\section{Space-charge effects}

The acceleration of a $20 \mathrm{psec}(\mathrm{FWHM}), 1 \mathrm{nC}$ bunch represents a 50-ampere instantaneous current with significant space charge defocusing force. The codes have been used to analyze the transport from the cathode to the end of a 3-cell structure. In these simulations, only the external rf fields in the cavities were present, no solenoidal focusing was yet present.

To study the effects of space charge the parameters were varied in two ways. First, the charge within the bunch was varied from $1 \mathrm{pC}$ to $1 \mathrm{nC}$ keeping all other parameters fixed. Figure 6-1 shows how the normalized transverse emittance varied, indicating a clear blow up at high charge as expected from space charge effects. MAFIA and PARMELA agree quite well at the exit of the first cell $(65 \mathrm{~mm})$ but differ at the gun exit $(300 \mathrm{~mm})$. 


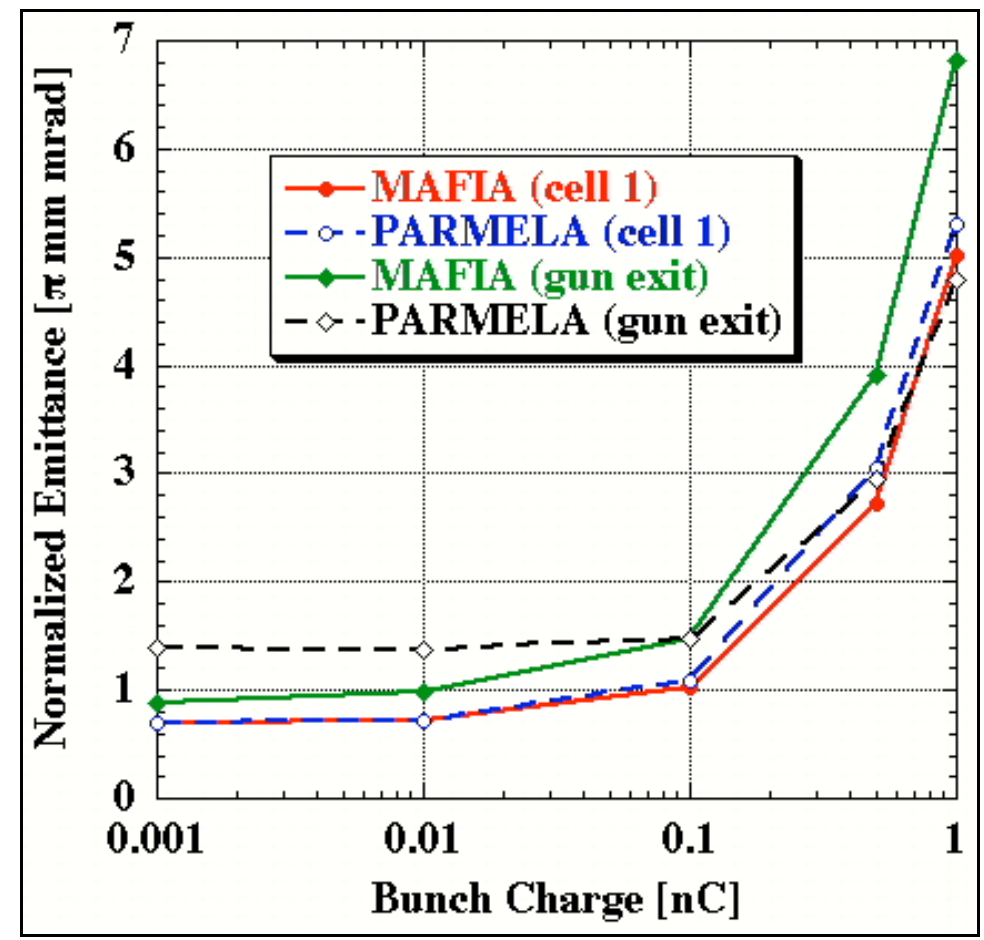

Figure 6-1 Variation of normalized transverse emittance with bunch charge, $64 \mathrm{MV} / \mathrm{m}$ on cathode, $60^{\circ}$ launch phase, 10 ps bunch length, $43 \mathrm{MV} / \mathrm{m}$ for cells 2 and 3.

The other method kept the charge fixed at $1 \mathrm{nC}$ and varied the bunch length from $10 \mathrm{ps}$ to $40 \mathrm{ps}$, thus reducing the charge density by up to a factor of four. Figure 6-2 shows how the transverse emittance from MAFIA varied over this range. Clearly there is less sensitivity to charge density in this case. At the end of the first cell $(65 \mathrm{~mm})$, the transverse emittance is seen to decrease monotonically with pulse length, as the longitudinal emittance increases linearly. That the longitudinal emittance increase linearly with pulse length in the early stages is expected since the energy spread has not yet had sufficient time to respond to longitudinal space charge or nonlinear rf forces. However, by the end of the rf gun, the effects due to increasing the bunch length become clearer. The transverse emittance shows a definite minimum as space charge forces lessen while nonlinear rf focusing increases. A similar trend is observed in the longitudinal phase space. The induced head-tail energy spread from space charge forces decreases with bunch length, while the nonlinear effects of the large rf phase range become more significant. However, we see that operation around the minima to decrease transverse emittance and space charge induced head-tail energy spread indicates a bunch length between 20 ps and 30 ps. 


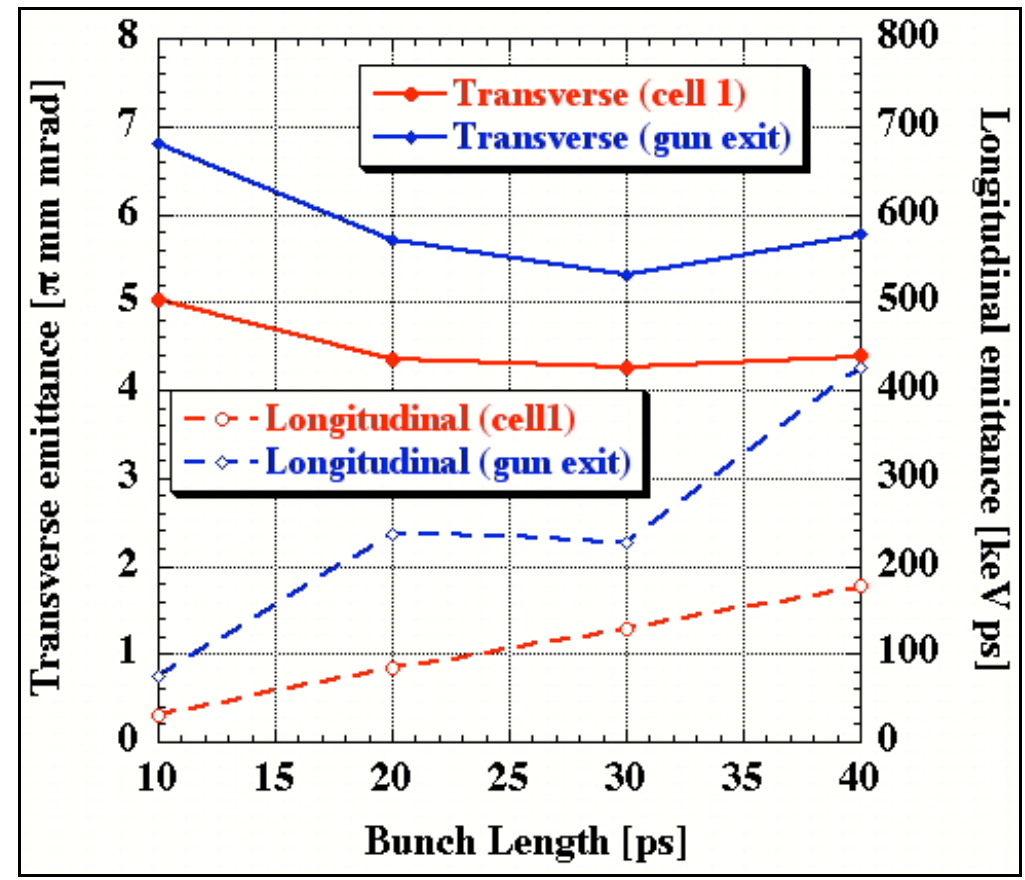

Figure 6-2 Variation of normalized transverse emittance with bunch length, $64 \mathrm{MV} / \mathrm{m}$ on cathode, $60^{\circ}$ launch phase, $1 \mathrm{nC}$ charge, $43 \mathrm{MV} / \mathrm{m}$ for cells 2 and 3 (MAFIA).

\section{Launch phase}

Another important parameter in the gun dynamics is the phase of the rf cycle at which the bunch is launched from the cathode. Simulations were run with launch phases from $10^{\circ}$ to $90^{\circ}$ with all other parameters fixed, see Figure 6-3. The optimum appears to be well ahead of crest. This may indicate that $\mathrm{rf}$ focusing is at least as important as accelerating field at the cathode.

Simulations show a similar trend in the longitudinal emittance after the first cell, although the codes differ on the final number. Bunching due to the slope of the rf waveform may be compensating for longitudinal space charge forces at low phase angle. The calculated longitudinal emittance leaving the last cell doesn't show a clear trend, however no attempt was made to optimize the relative phasing of the later cells for different launch phases.

\section{Phase offset between first and second cells}

Introduction of the short drift between the gun cell and the subsequent accelerating cells introduces a small timing offset. Retarding the downstream cells by about $30^{\circ}$ can compensate this. However, simulations indicate that apart from a small increase in the energy there is not much effect on the output beam parameters when this is added. 


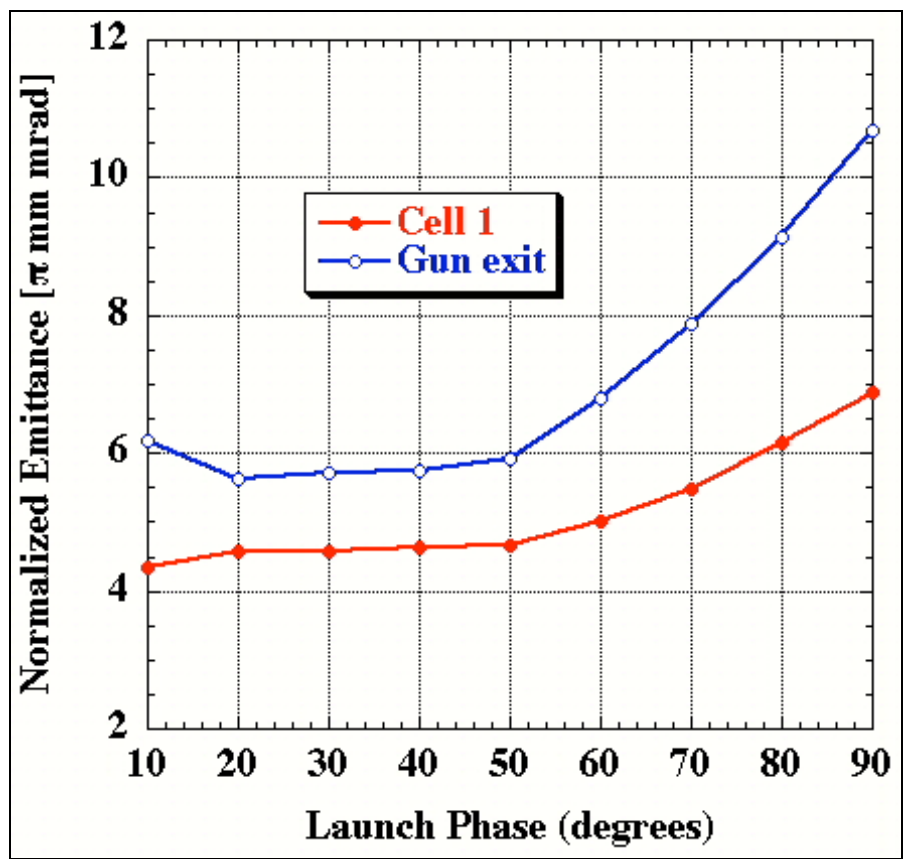

Figure 6-3 Variation of normalized transverse emittance with launch phase, $64 \mathrm{MV} / \mathrm{m}$ on cathode, $1 \mathrm{nC}, 10 \mathrm{ps}$ bunch length, $43 \mathrm{MV} / \mathrm{m}$ for cells 2 and 3 (MAFIA).

\section{SOLENOID COMPENSATION OF EMITTANCE}

Emittance compensation techniques have been thoroughly described in the literature [4,5]. Briefly, emittance compensation is the process by which the transverse phase space profiles of different slices along the bunch are made to re-align in a coherent way so as to reduce the sum, or projected, emittance of all the slices to its minimum value. This minimum value is bounded from below by the initial thermal emittance.

These techniques are employed to deliver the lowest possible transverse emittance at a specified point downstream from the exit of the rf gun. Typically, this point is made to coincide with the entrance of a subsequent accelerating structure. Shown in Figure 6-4 below is the result from a HOMDYN simulation of the 3-cell rf gun, without solenoidal focusing (uncompensated) and with solenoid coils excitation (compensated). In the compensated case, the solenoids have been tuned to produce a beam that exhibits very little projected emittance growth in the drift region nearly two meters past the end of the rf gun. This particular configuration does not generate the minimal normalized transverse emittance possible in the drift region. However, the very long beam waist achieved may be more beneficial for matching into a downstream skew quadrupole lattice. This issue is currently being considered. 


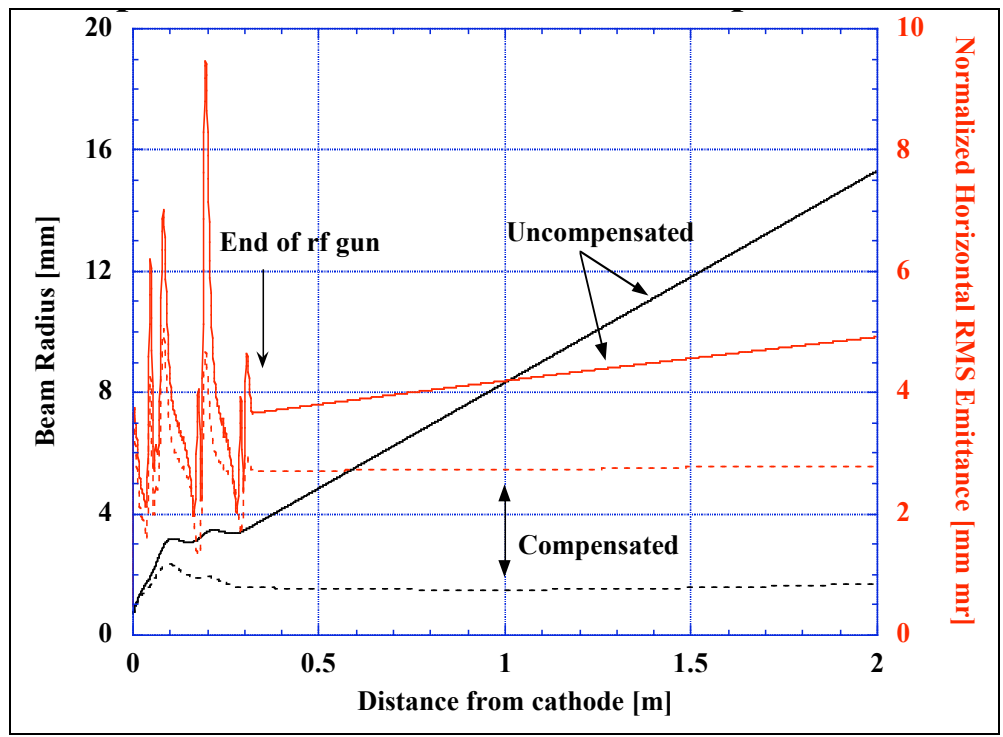

Figure 6-4 HOMDYN model of uncompensated (solid) and compensated (dashed) beam dynamics in the rf gun and drift section.

The flat beam photoinjector differs from previously studied rf photoinjectors in a fundamental way. Previous designs were concerned with propagating a beam through an azimuthally symmetric channel, at least as far as the exit of the pre-accelerators when space charge effects were no longer relevant to the detailed beam dynamics. Hence, symmetry was maintained until it no longer mattered. Actually, this is true for the majority of electron injectors, with the exception of sheet beam klystrons. For the proposed photoinjector, however, we must design a beamline that intentionally breaks the azimuthal symmetry by the introduction of skew quadrupoles in a region where space charge effects are still important. Moreover, while azimuthally symmetric photoinjectors strive to produce beams with emittances in both the horizontal and vertical planes of less than $1 \pi \mathrm{mm}$-mrad for a $1 \mathrm{nC}$ bunch charge, we need only produce a beam that has a geometric-averaged emittance of $\sim 3 \pi \mathrm{mm}$-mrad for a $1 \mathrm{nC}$ bunch charge. However, we must do this while producing a high ratio ( $>50-100: 1)$ between the horizontal and vertical emittances at the end of the injector. We must eventually produce a beam with vertical emittance approximately equal to the initial, thermal emittance value or less. Hence, our strategy for emittance compensation will differ in some respects from the already established technique.

\section{Theoretical Considerations}

Previous emittance compensation studies have assumed that the beam is emitted at a location free from magnetic fields, and that any contribution to the beam envelope due to canonical angular momentum is negligible. In this study, we intentionally apply a solenoid field such that lines of magnetic flux thread the cathode. The envelope equation describing the evolution along 
the beamline, parameterized by distance $(z)$, of the RMS radial beam spot size $\left(\square_{r}=\sqrt{\left\langle r^{2}\right\rangle}\right)$ can be expressed as [6]

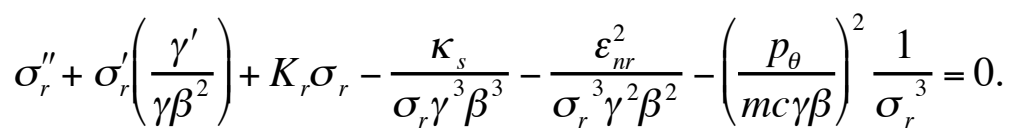

where the second term represents the focusing effect from acceleration. The third term multiplier, $K_{r}=\frac{e B_{z}}{2 \square m c}$, represents the influence of linear focusing in a solenoidal magnetic channel. The fourth term multiplier, $\square_{s}=2 I / I_{0}$, represents the defocusing influence of space charge effects, with $I_{0}=4 \square m c / \square_{0} e \square 17 k A$. The final two terms represent the defocusing influence of finite radial emittance and finite canonical angular momentum, which can be seen to influence the envelope dynamics in an equivalent manner.

The emittance term may be evaluated by several means [7]. In an azimuthally symmetric system, the total 4-D transverse emittance can be expressed as

$$
L_{h}=\frac{1}{4}(\square)^{2}\left[\left\langle r^{2} X \square^{2}+(r \square \square)^{2}\right\rangle \square\left\langle r \cdot r \nabla^{2} \square\left\langle r^{2} \square\right\rangle^{2}\right],\right.
$$

where $r \square=d r / d z, \quad[Z=d \square / d z$. This emittance represents the volume of the full 4-D transverse phase space and may be written in terms of the component emittances describing the radial and angular degrees of freedom,

$$
\begin{aligned}
& L_{h \mathrm{r}}=\frac{1}{4}(\square)^{2}\left[\left\langle r^{2}\right\rangle\langle r \square\rangle \square\left\langle r \cdot r \square^{2}\right]\right. \\
& \square_{h_{\square}}=\frac{1}{4}(\Pi)^{2}\left[\left\langle r^{2}\right\rangle\left\langle(r \square)^{2}\right\rangle \square\left\langle r^{2} \square\right\rangle^{2}\right] \text {. }
\end{aligned}
$$

For rigid rotation (constant $\nwarrow$ ), the last expression is identically zero. We may ignore the emittance in the angular degree of freedom, as this does not directly affect the evolution of the beam envelope until the beam enters the skew quadrupole channel.

The equation of motion for the angular degree of freedom of each particle can be expressed as the rate of the Larmor phase angle advance

$$
\square \square=\frac{p_{\square}}{\llbracket m c r^{2}}+\frac{e B_{z}}{2 \llbracket m c}
$$

In the absence of canonical angular momentum, the Larmor phase angle advances at one-half the rate of cyclotron phase angle.

This last expression is important to consider for the flat beam injector. The conditions at the cathode plane present initial conditions for the solution of this equation of motion. In the case of a typical injector, the magnetic field is designed to be null at the cathode plane. For this case, $B_{z}(z=0)=B_{z 0}=0$. From knowledge of the initial conditions at the cathode, we know that $\dot{\square}(z=0)=\square c \square(|l| z=0)=0$ and, hence, $\quad p_{\square}($ all $z)=0$. This last relation follows from global 
conservation of angular momentum. In the case of the flat beam injector, a finite magnetic field is required at the cathode plane. The conserved angular momentum for a particle emitted at radius $r_{0}$ is then

$$
p_{\square}=\square \frac{1}{2} e B_{z 0} r_{0}^{2} .
$$

The angular equation of motion determines the trajectory of beam particles in the angular phase-space. It depends upon the local particle radius, to which it is coupled by any finite canonical angular momentum. However, the radial envelope equation (as well as the individual particle's radial equation of motion) does not depend on the detailed evolution of the angular degree of freedom, so long as the beam remains azimuthally symmetric. This will be the case for the beams under consideration here, until they reach the entrance of the skew quadrupole transformer.

The radial envelope equation above describes the evolution of the radial degree of freedom of the ensemble of particles comprising the beam. The initial angular momentum contributes to the evolution of the beam envelope in the same way as the emittance. The effective radial emittance is then the sum in quadrature of the radial emittance $\left(C_{h r}\right)$ and the influence of the canonical angular momentum [7],

$$
\bar{\zeta}_{h r}^{f f}=\sqrt{\left(\square_{h r}\right)^{2}+\frac{1}{4}\left\langle\frac{p_{\square}}{m c}\right\rangle^{2}} .
$$

We may then study solutions to the envelope equation in the context of the design of the proposed rf gun by parameterizing with respect to the initial angular momentum. The rf gun design may then be considered optimized for a particular value of the initial angular momentum when the effective radial emittance is minimized. For the eventual generation of flat beams with large emittance ratio, we will see that large values of the angular momentum are required along with preservation of the radial emittance to values close to that of the initial thermal emittance.

\section{Large And Small Angular Momentum Regimes}

We have used the HOMDYN code to evaluate possible operating points of the flat beam injector. These operating points are defined by the set of parameters: solenoid coil excitations (solenoid tune), laser spot size at the cathode. For the proposed 4-cell, 5-solenoid rf gun, this presents a 6-D parameter space. The set of solenoid coil excitations define the solenoid magnetic field at the cathode, and the cyclotron phase advance once the rf fields and beam launch phase are given. The laser spot size at the cathode determines the thermal emittance, the canonical angular momentum (once the solenoid field at the cathode is known), and provides the starting point for solution of the envelope equation and for calculating the Larmor phase advance. By searching the available parameter space, we can make some initial predictions of the performance of the rf gun portion of the injector.

For any set of solenoid fields used, the degree of emittance compensation provided by solenoid focusing can be measured against the cyclotron phase advance $\square_{c}$, defined by 


$$
\square_{c}\left(t_{\text {final }}\right)=\prod_{0}^{t_{\text {final }}} d t \frac{e B_{z}}{m \square} .
$$

This parameter is only useful when comparing simulations or experimental data sets when the laser spot size incident on the photocathode is held constant from one case to another. In the general case where the spot size is also allowed to vary, the more general Larmor phase advance given by

$$
\square_{L}\left(t_{\text {final }}\right)=\square_{0}^{t_{\text {final }}} d t \stackrel{\square}{\square} \frac{p_{\square}}{m \square r^{2}}+\frac{e B_{z}}{2 m \square}
$$

is more appropriate. The utility of the cyclotron phase advance is such that it only depends on the solenoid tune and the beam energy. It provides a measure independent of the beam envelope dynamics, including space charge and emittance effects.

Below we present results that have been parameterized by the initial angular momentum in units of emittance ( $\pi \mathrm{mm}$-mrad). These simulations show the solution of the envelope equation of the beam as it is emitted from the cathode, passes through the rf gun, and exits the region of solenoid field to the drift section beyond. We do not consider the skew quadrupole channel here, except to match the beam envelope to the entrance of the channel located approximately $1 \mathrm{~m}$ from the cathode plane $(z=0 \mathrm{~m})$.

At the cathode plane, the thermal emittance is determined by the product of the laser spot size with the distribution of the transverse electron momenta,

$$
C_{h r}^{\text {hermal }}=\frac{1}{2}(\Pi)_{\text {cathode }} \sqrt{\left\langle r^{2}\right\rangle_{\text {cathode }}\left\langle r^{\square}\right\rangle_{\text {cathode }}} .
$$

For $\mathrm{Cs}_{2}$ Te cathodes, HOMDYN uses an analytic model [8] for the thermal emittance, approximately $0.7 \pi \mathrm{mm}$-mrad per $\mathrm{mm}$ of initial rms beam radius.

We have identified two regimes of operating points. The first regime occurs for small values of angular momentum $(<\sim 15 \pi \mathrm{mm}$-mrad $)$ and thermal emittance $(\sim 0.7 \pi \mathrm{mm}-\mathrm{mrad})$. A typical solution (with angular momentum contribution $\sim 8 \pi \mathrm{mm}-\mathrm{mrad}$ ) is shown in Figure 6-5. This regime can achieve optimized solutions that demonstrate radial emittance $\sim 3 \pi \mathrm{mm}$-mrad and very long beam waists. While the radial emittance has grown to several times that of the initial thermal value, it still remains within the design specifications. The beam envelope and radial emittance remain relatively constant over nearly $2 \mathrm{~m}$ of drift, which may be advantageous for matching into the flat beam adapter lattice.

The second regime occurs for larger angular momentum $(\sim 20-50 \pi \mathrm{mm}-\mathrm{mrad})$, and larger thermal emittance $(\sim 1-2 \pi \mathrm{mm}-\mathrm{mrad})$. This regime is characterized by larger spot sizes (up to $\sim 3 \mathrm{~mm}$ rms radius) to generate the larger initial angular momenta, and overall larger excitation of the focusing solenoids. The results of two optimized solutions in this regime are presented in Figure 6-6. The resulting envelope solutions show minima in radial emittance at the adapter entrance $(z \sim 1 \mathrm{~m})$ that exhibit only small growth $(\sim 10 \%)$ above the initial thermal emittance value. 
These two simulations demonstrate the feature of emittance oscillation and compensation. Both simulations demonstrate the radial emittance undergoing oscillations. The first of the minima is located at the cathode $(z=0 \mathrm{~cm})$, with the next local minima located (by careful optimization of the solenoid focusing channels) approximately $1 \mathrm{~m}$ from the cathode.

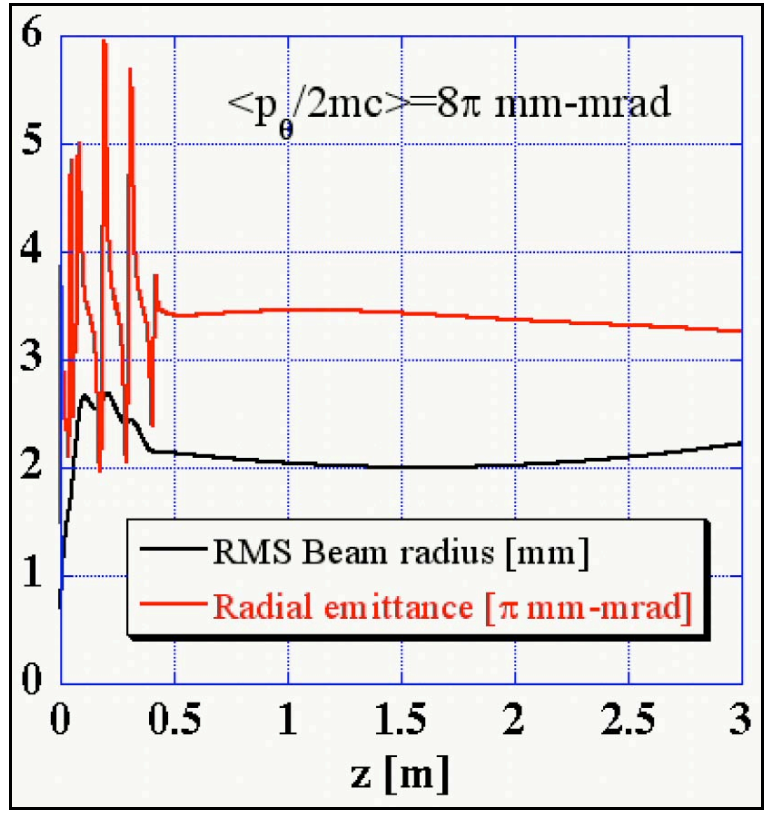

Figure 6-5 Small angular momentum regime (HOMDYN).

These solutions may be more suitable for the flat beam injector, provided they can be matched into the skew quadrupole channel. We will consider working points in the large angular momentum regime below when we discuss the initial design of the flat beam adapter for the proposed injector. 


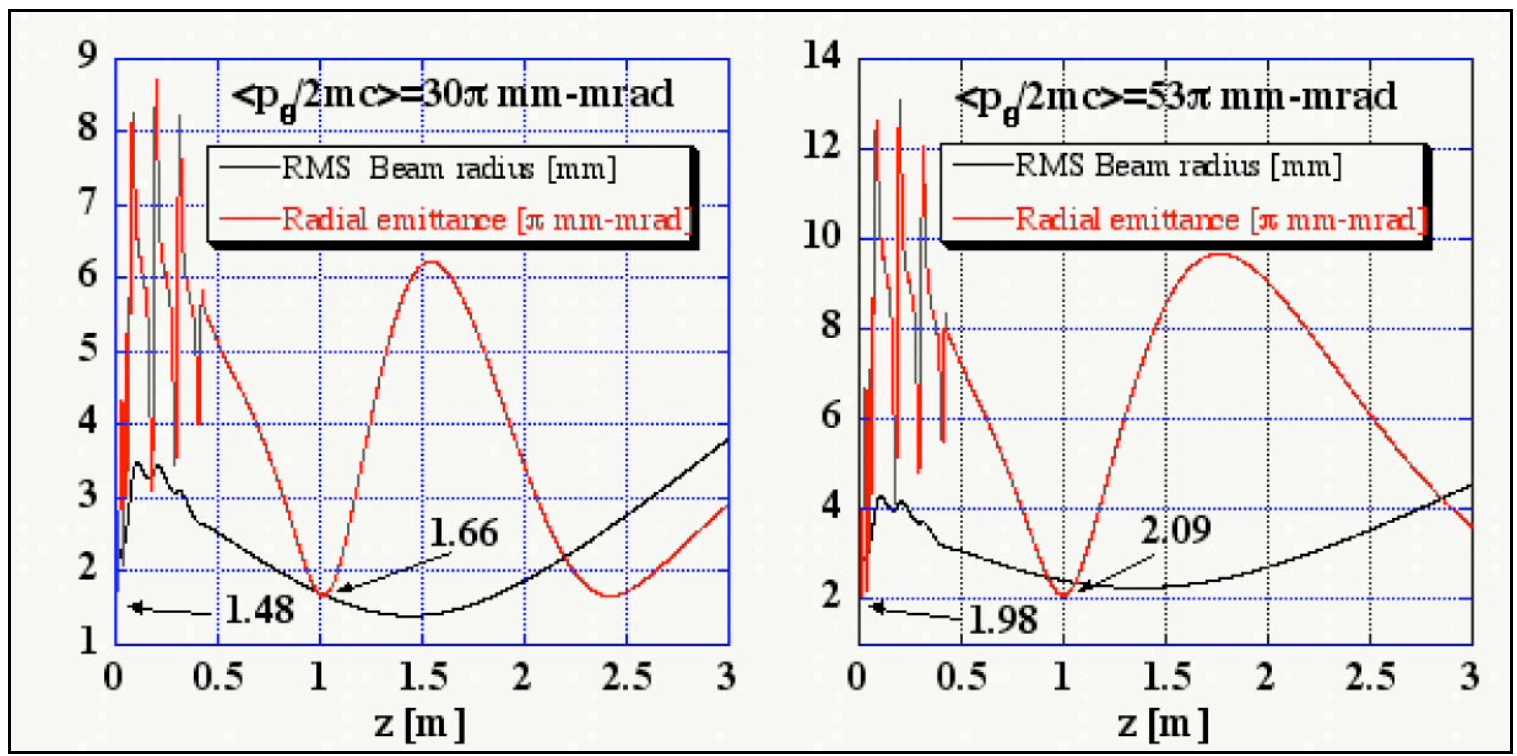

Figure 6-6 The initial thermal emittance and the projected emittance at the adapter entrance are indicated (HOMDYN)for the large angular momentum regime.

\section{REFERENCES}

[1] M. Ferrario, et al., 'Multi-bunch energy spread induced by beam loading in a standing wave structure', Particle Accelerators, 52, 1 (1996).

[2] L. Young and J. Billen, PARMELA code manual, LANL Report No. LA-UR-96-1835 (Revised), 2002.

[3] R. Rimmer, et. al., 'A High-Gradient, High-Duty-Factor rf Photo-Cathode electron Gun', to appear in the Proceedings of the Eighth European Particle Accelerator Conference (EPAC 2002), Paris, France, 2002.

[4] B.E. Carlsten, Nucl. Instrum. Methods Phys. Res. A 285, 313 (1989).

[5] L. Serafini and J.B. Rosenzweig, 'Envelope analysis of intense relativistic quasilaminar beams in rf photoinjectors: A theory of emittance compensation', Phys. Rev. E, 55, 7565 (1997).

[6] M. Reiser, Theory and Design of Charged Particle Beams (Wiley, New York, 1994).

[7] S. Nagaitsev and A. Shemyakin, 'Beam emittance calculation in the presence of an axially symmetric magnetic field', FERMILAB-TM-2107, 2000.

[8] K. Flöttmann, 'Estimation of the thermal emittance of electrons emitted by Cesium Telluride photo cathodes', TESLA-FEL-97-01, DESY-Hamburg, 1997. 


\section{EXPERIMENTAL STUDIES AT FNPL}

To pursue flat beam production techniques, we have been conducting experimental studies of rf gun operation and flat beam production in collaboration with personnel attached to the FNPL (Fermilab-NICADD Photoinjector Laboratory) facility [1]. The layout of the FNPL beamline is shown in Figure 7-1.

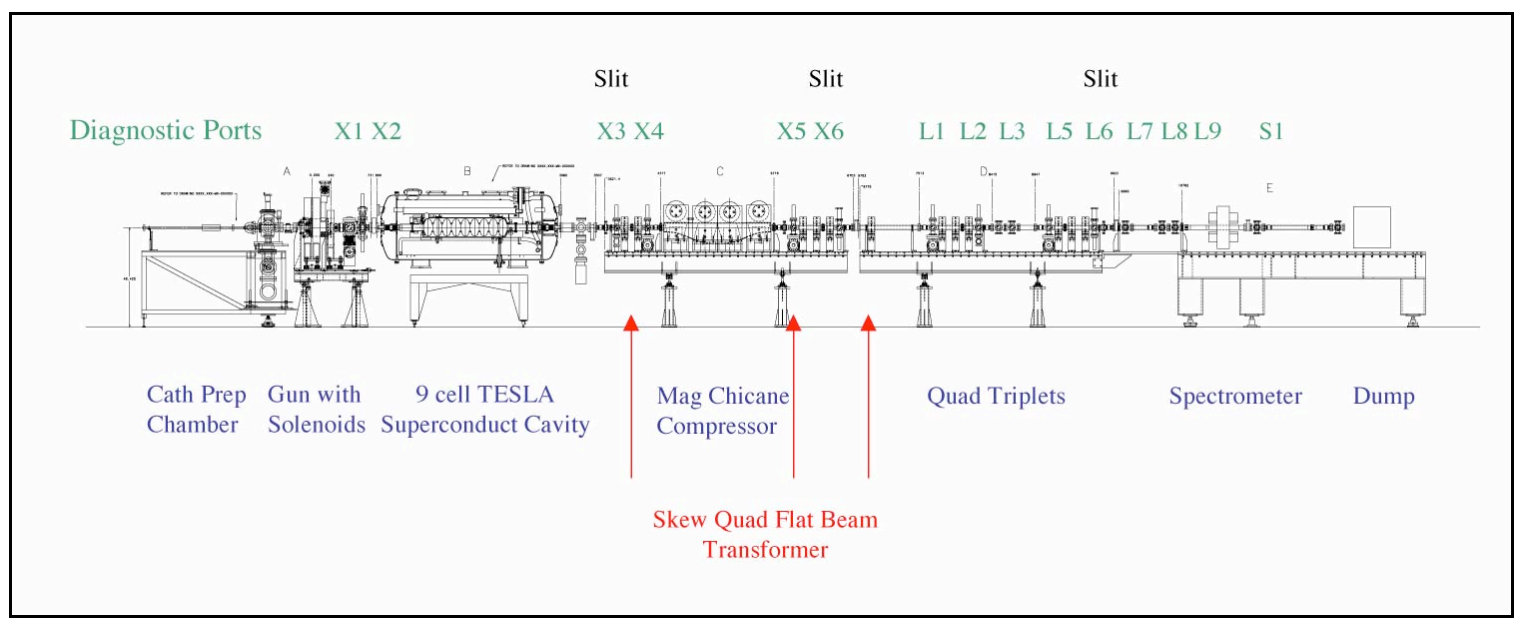

Figure 7-1 FNPL beamline.

The FNPL photoinjector consists of a 1.6 cell, $1.3 \mathrm{GHz}$ rf gun with a high quantum efficiency $\mathrm{Cs}_{2}$ Te photocathode driven by a frequency-quadrupled $\mathrm{Nd}$ :glass laser, followed by a 9-cell superconducting booster cavity resulting in an 14-15 MeV electron beam. A dipole chicane is present to provide magnetic bunch compression. The parameters of the photoinjector are summarized in Table 7-1.

Following the booster cavity are two sections of beamline for bunch-length compression and beam optical function matching. These sections are instrumented with diagnostic foils, optics, and CCD imaging cameras to record the beam spot at various locations along the beamline. Additionally, at several locations along the beamline, there are horizontal and/or vertical multislit analyzers that may be lowered into the path of the beam. When used in conjunction with downstream imaging foils, these analyzers can measure the beam's emittance in the horizontal or vertical planes. During flat beam operation, these same analyzers may be used to measure the angular momentum of the beam.

As shown in Figure 7-1, three of the normal quadrupoles have been rotated about their axis to become skew quadrupoles. During flat beam production, only these quadrupoles are energized. 
Table 7-1 FNPL Photoinjector parameters

\begin{tabular}{|ll|}
\hline RF $1.3 \mathrm{GHz}$ drive & $30-600 \square \mathrm{sec} @ 1 \mathrm{~Hz}$ \\
Typical Q.E. & $\sim 0.5-2 \%$ \\
Laser UV Energy & $1-5 \square \mathrm{J} /$ bunch \\
Bunch charge & $1-10 \mathrm{nC}$ \\
Total Energy after gun & $4.5-5 \mathrm{MeV}$ \\
Total Energy after booster & $14-15 \mathrm{MeV}$ \\
Compressor dl/(dp/p) $\left(\mathrm{R}_{56}\right)$ & $84 \mathrm{~mm}$ \\
Gun solenoids & $1200 \mathrm{Gauss}$ peak typical \\
Gun gradient & $35-40 \mathrm{MV} / \mathrm{m}$ on cathode \\
Eaco, $_{\text {a-Cell SC cavity }}$ & $10 \mathrm{MV} / \mathrm{m}$ \\
Macro-pulse repetition rate & $1 \mathrm{~Hz}$ \\
Micro-pulse structure & $1 \square \mathrm{sec}$ bunch spacing \\
\# Bunches per macro-pulse & $1-800(1-20$ typical $)$ \\
\hline
\end{tabular}

\section{EXPERIMENTAL STUDIES OF OPTIMIZATION OF TRANSVERSE EMITTANCE}

The FNPL injector was designed and built to generate high brightness round beams. The 1.6 cell $\mathrm{rf}$ gun is equipped with three solenoids to provide focusing and emittance compensation tuning. The first two coils, the bucking and the main, provide focusing in the region immediately downstream from the cathode while ensuring that no magnetic flux lines actually thread through the cathode surface - effectively zeroing any angular momentum contribution to the effective emittance. The third coil, the secondary, is located near the gun exit, and provides most of the tuning capability for emittance compensation and optical function matching downstream.

Parametric studies have been performed to examine the effect of solenoid focusing on flat beam final emittance and emittance ratio. Two parameters were varied - the current in the main solenoid and the current in the secondary solenoid. The main solenoid provides the field on the cathode and defines the initial canonical angular momentum on the beam. The secondary solenoid controls the matching into the booster accelerator. The remaining, bucking, solenoid was not used during these studies.

These studies attempt to address two distinct, though related, goals concerning the operation of high brightness injectors intended for flat beam production. The first goal has been to explore and contrast the differences that exist in successful space charge emittance compensation techniques used on pre-existing high brightness photo injectors and those that produce angular momentum dominated beams. That is, we seek to determine the requirements of the focusing solenoid channel in order to obtain a specified angular momentum at the photocathode such that it generates a beam with the lowest radial emittance. The second goal, once the first has been achieved, is the determination of the optimum placement of the skew quadrupole channel entrance. That is, we seek to determine how a beam becomes matched to the skew quadrupole lattice such that its four dimensional emittance is conserved and the lowest emittance in one plane is achieved. Studies to date have focused on the first of these goals. 
The cyclotron phase advance at the entrance to the booster module has been calculated using HOMDYN. Figure 7-2 shows the cyclotron phase advance (in units of $\square$ ) as the secondary solenoid current is varied over its full range, for different values of the main solenoid current. By varying the current in both the main and secondary solenoids a usable range of cyclotron phase advance from $\sim 0.8 \square$ to $\sim 1.5 \square$ radians can be explored. Additionally, we find three phase advance curves that each have $\sim 1.12 \square$ radians in their range, so that this point can be explored with three different settings of the main solenoid and, hence, three different values of canonical angular momentum.

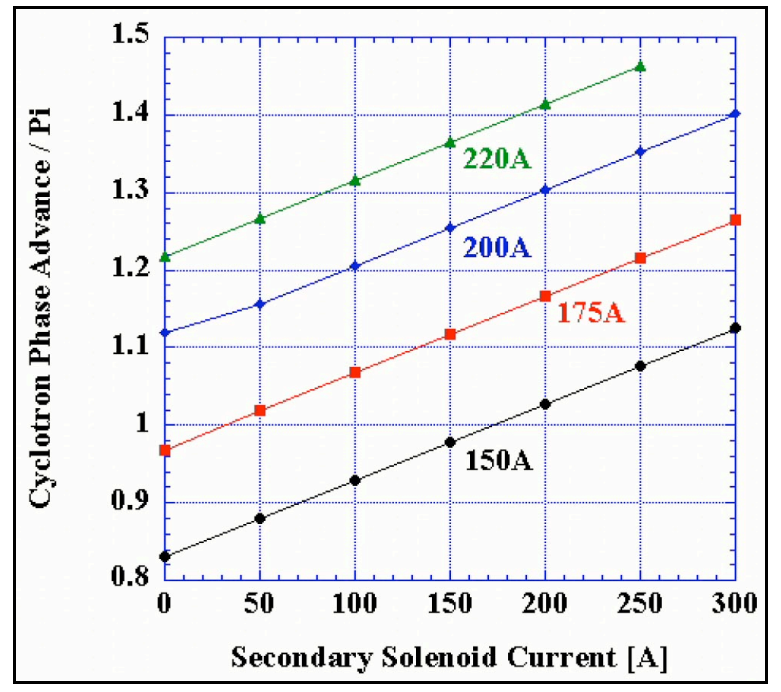

Figure 7-2 Cyclotron phase advance (in units of $\pi$ ) vs. secondary solenoid current (HOMDYN).

Over the studied range of cyclotron phase advance, we have identified four distinct regions: $<1 \square, \sim 1 \square, \sim 1.35 \square$, and $>1.4 \square$. Beam envelopes and radial emittances from HOMDYN simulations for these regions $(0.88 \square, 1.1 \square, 1.35 \square, 1.46 \square)$ are shown in Figure 7-3. 


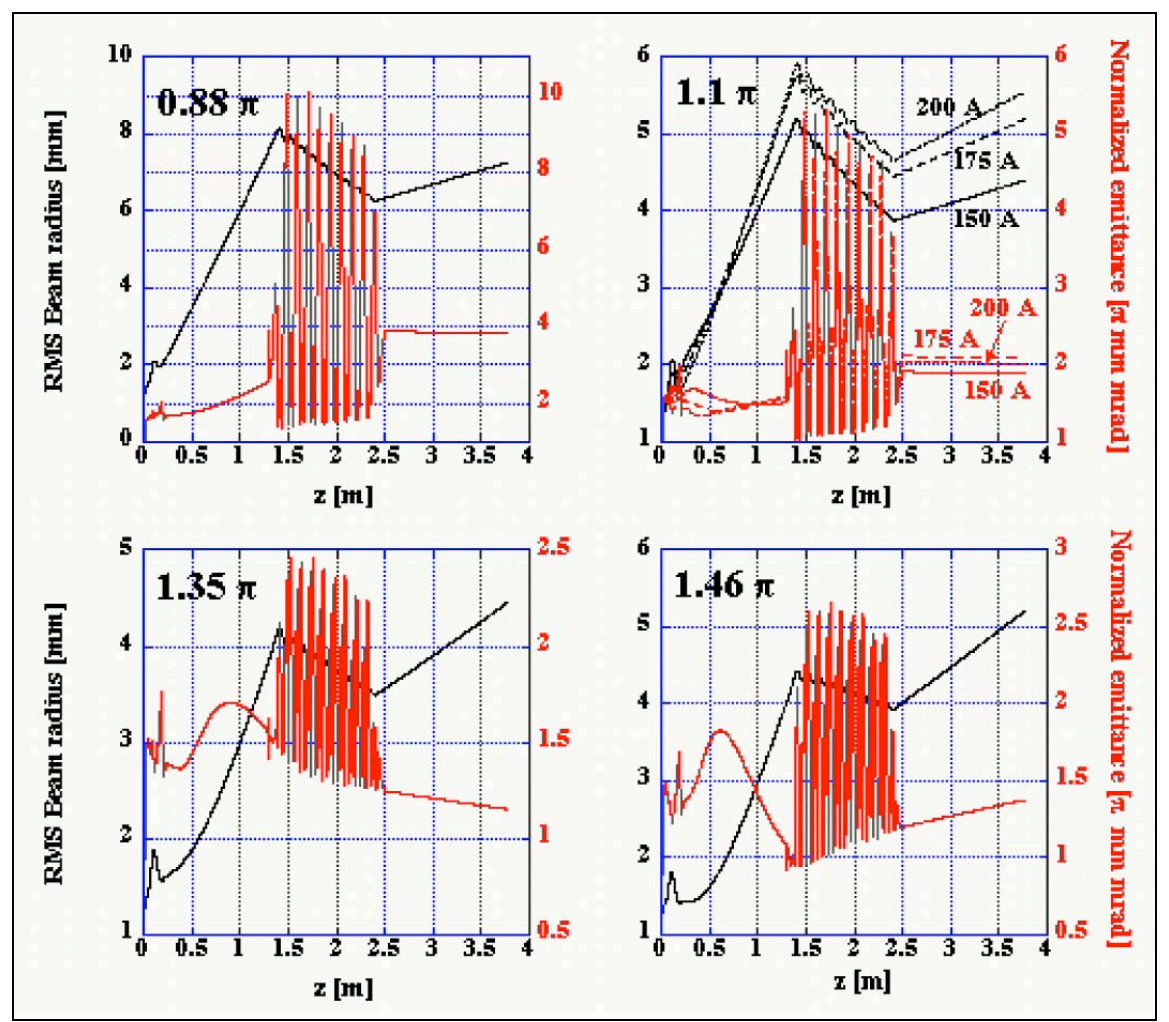

Figure 7-3 Beam envelope (black) and radial emittance (red) for cyclotron phase advances of $0.88 \pi, 1.1 \pi, 1.35 \pi$, and $1.46 \pi(H O M D Y N)$.

In the first region, with cyclotron phase advance of $0.88 \pi$ (main solenoid current of $150 \mathrm{~A}$ and secondary solenoid current of $50 \mathrm{~A}$ ), the beam is under-compensated, and space charge effects monotonically increase the radial emittance before the beam enters the booster cavity.

In the second region $(1.1 \pi)$, the beam is roughly compensated and matched into the booster. The radial emittance at the exit of the booster shows a reduction in half compared to the previous case. We see here the utility of cyclotron phase as a measure of emittance compensation. The plot shows the results of three separate simulations, with different excitation currents for the main and secondary solenoid coils (150A/300A, 175A/150A, and 200A/0 A, respectively). The solenoid field at the cathode is primarily dependent on the main coil excitation, with the secondary coil responsible for matching the beam optics downstream. The difference in the main coil setting results in differences in the beam envelope downstream. Nevertheless, the equivalent cyclotron tune ensures that the radial emittance following the booster cavity is nearly equal.

The third $(1.35 \pi)$ and fourth $(1.46 \pi)$ cases show the over-focused (over-compensated) case, where oscillations in the radial emittance are noticeable. There exists a local maximum in the radial emittance in both cases prior to injection into the booster cavity. In the third case, the projected radial emittance is still evolving towards the next minimum in the region following the booster cavity. In the fourth case, meanwhile, the larger degree of over-focusing enables the second minimum to be located at approximately the booster cavity entrance. 
By tuning the solenoids in the rf gun, the location of the second minimum can be placed downstream of the booster cavity at the entrance to the skew quadrupole adapter lattice. At this location, the results for the radial emittances are calculated and plotted as a function of cyclotron phase advance in Figure 7-4. We see a minimum in the radial emittance at cyclotron phase advances $\sim 1.35 \pi$. The region of overlapping cyclotron phases occurs for phase advances $\sim 1.1 \pi$

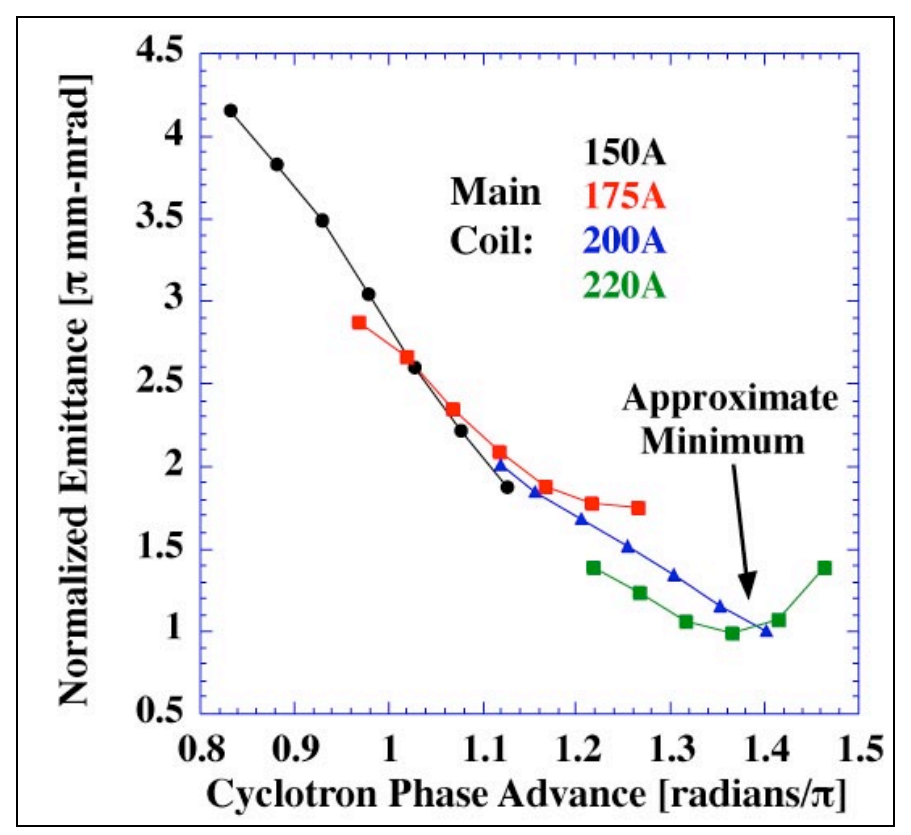

Figure 7-4 Vertical emittance vs. cyclotron phase advance (HOMDYN). The overlapping phases at $\sim 1.1 \pi$ are described in the text.

\section{Vertical Emittance Measurement}

The vertical emittance at the entrance to the skew quad channel has been measured using the horizontal slit analyzer at the booster cavity module exit and the imaging the beamlets on downstream optical transition radiation (OTR) foils. Measured values are shown in Figure 7-5. Over the range of parameters scanned, and by comparison with Figure 7-2, we see that the individual emittance minima occur for nearly equal values of the cyclotron phase advance as calculated by HOMDYN, with an absolute minimum seen when the main solenoid is set to $150 \mathrm{~A}$.

The absolute values of the emittance are properly recognized as upper bounds, as they reflect the projected emittance averaged over approximately 20 bunches with varying bunch charge, and hence varying compensated emittance values. One near-term goal of the experimental program at FNPL is to commission diagnostics capable of measuring the spot size and slit profiles with adequate signal-to-noise to permit an emittance measurement of a single bunch. 


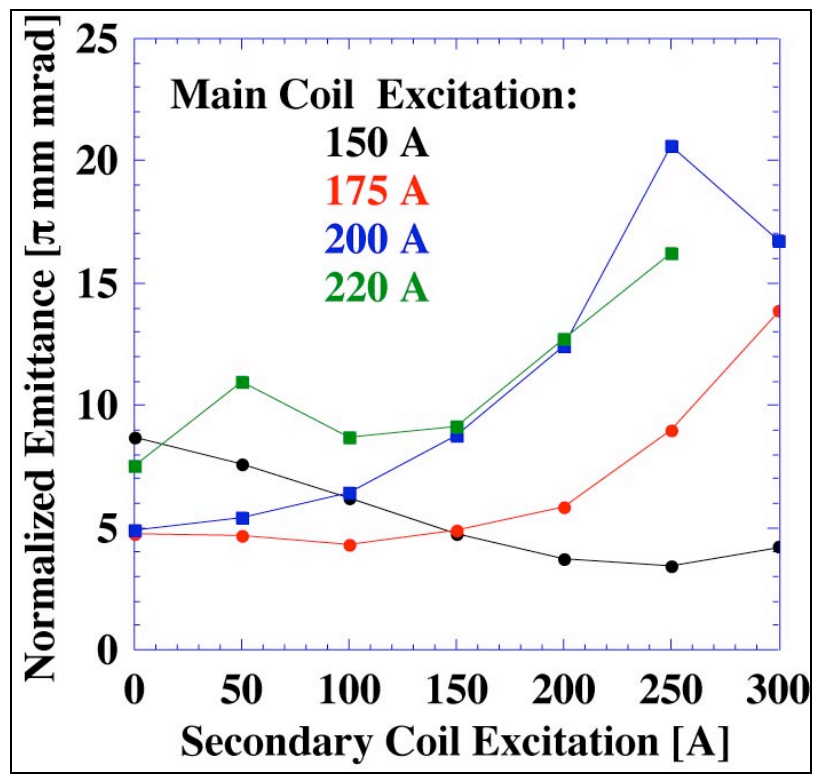

Figure 7-5 Measured vertical emittances of angular momentum-dominated beam at FNPL.

\section{Future Studies of EmitTance Compensation}

We will continue our studies of emittance compensation in the angular momentum dominated regime. The first task is to evaluate with simulation the performance of small and large angular momentum regimes when combined with adapter lattice optics. This can be completed in two separate stages: one is to determine the solenoid tunes which optimize the beam transport (with respect to radial emittance) for a given value of the canonical angular momentum; the other is to determine the required beam optical functions at the entrance to the adapter. When these two sets are combined an overlapping solution can then be identified. Different adapter lattices will be explored that may add additional flexibility to matching the incoming beam.

We will continue general sensitivity studies of systematic errors. These systematic errors include tuning errors in the solenoids and quadrupoles, laser spot transverse and temporal uniformity deviations, and nonlinear optical and space charge effects in the beam transport. The effects of rf gun errors including field balance, and amplitude, phase, and timing jitter will be examined as well.

We will continue to work with personnel at FNPL in developing better data sets as the laser system and diagnostics are improved. This will enable the exploration of larger regions of the available parameter space, and to conduct studies of emittance sensitivity to parameter variations.

\section{ReferenCES}

[1] J.P. Carneiro, et. al., 'Beam transport, acceleration and compression studies in the Fermilab high-brightness photoinjector', Proceedings of the XIX International Linac Conference, Chicago, 1998. 


\section{FLAT BEAM ADAPTER}

The flat beam adapter transformation of an angular momentum dominated was first proposed in the context of electron cooling of high-energy hadron beams [1]. The concept has subsequently been more thoroughly described [2,3] as well as applied to the problem of generation of flat electron beams [4]. In this section, we present a brief overview of the process, and then present an initial design of the optics that achieve the requirements of the proposed facility.

\section{GENERATION OF THE BEAM IN A SOLENOID FIELD}

Electrons are emitted from the photocathode surface into a region of large $(\sim 1 \mathrm{kG})$ solenoidal magnetic field. In the absence of thermal emittance, or finite spread in transverse velocities, the individual electrons would follow paths along the lines of magnetic flux. The influence of a thermal spread in transverse velocities forces the electrons to precess about the magnetic flux lines. The equations of motion for the electrons in this transport channel can be written in socalled 'normal form' that decouples the 4-D transverse phase space into independently evolving 2-D phase spaces. The normal mode variables in this region are drift-cyclotron or 'Larmor' coordinates. This is a 'guiding center' description of the motion of particles in a solenoidal field, which describes the motion of particles not in terms of the absolute Cartesian coordinates $(x, x \rrbracket y, y \square$ or the cylinder coordinates $\quad(r, r \llbracket \square, \square \backslash]$, but rather in terms of the 'drift' position $\left(d_{x}, d_{y}\right)$ of the Larmor center and 'cyclotron' rotation $\left(k_{x}, k_{y}\right)$ about the Larmor center (see Figure 8-1). The Larmor coordinates are constructed via the relations

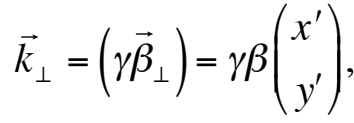

$$
\begin{aligned}
& \vec{d}=\left(\vec{r} \square \square_{L}\right)=\square_{y} x \square \frac{\square_{s}}{2}=y \square x
\end{aligned}
$$

Here, $\square_{s}=e B_{z} /(2 \square m c)$ is the equivalent beta-function in the solenoid channel.

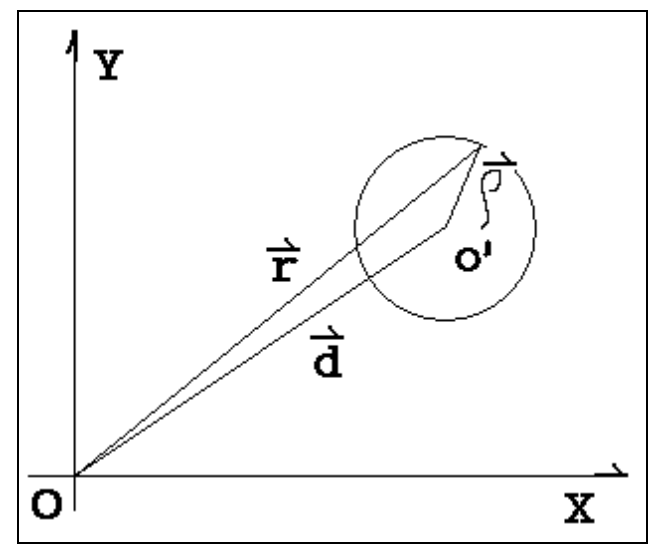

Figure 8-1 Drift-cyclotron coordinates 
It has been shown that the drift-cyclotron coordinates form canonically conjugate pairs [18], after proper normalization. The 4-D total transverse emittance is constructed from the independent sub-spaces, $\left(d_{x}, d_{y}\right)$ and $\left(k_{x}, k_{y}\right)$, as

$$
\square_{h}=\frac{1}{4}\left\langle d^{2}\right\rangle\left\langle k_{\square}^{2}\right\rangle=\square_{\text {rifit }} L_{\text {yclotron }},
$$

This is identical to the form described above, but specifically separates the contributions arising from the distinct normal modes. These two contributions can be quantified from parameters arising from conditions at the cathode surface during the emission process. The spot size of the beam at the cathode can be seen to determine the emittance in the drift plane, since $\left\langle d^{2}\right\rangle$ is proportional to the rms beam spot radius. Finite thermal emittance, originally arising from a distribution of electron velocities with finite spread, is immediately seen to contribute the cyclotron emittance that is proportional to the transverse velocity spread. Depending upon the beam spot radius, the thermal emittance for a $\mathrm{Cs}_{2}$ Te photocathode may vary from $\sim 0.5$ to $1.5 \pi$ mm-mrad. With a solenoid field at the cathode of magnitude $\sim 1 \mathrm{kG}$, the drift motion emittance may then be much larger than the cyclotron motion emittance. This can also be stated simply that the beam radius is much larger than the characteristic Larmor radius of the individual particle trajectories. This large ratio between drift and cyclotron emittances will be converted to a large ratio between horizontal and vertical emittances following the skew quadrupole adapter.

\section{EXTRACTION FROM THE SOLENOID FIELD}

Exiting the fringe field prepares the beam for the transformation in the skew quadrupole adapter. As the beam passes through the fringe field region at the end of the solenoid lattice, each particle is deflected by the radial magnetic field component. The Cartesian phase-space components are transformed in the thin lens approximation as

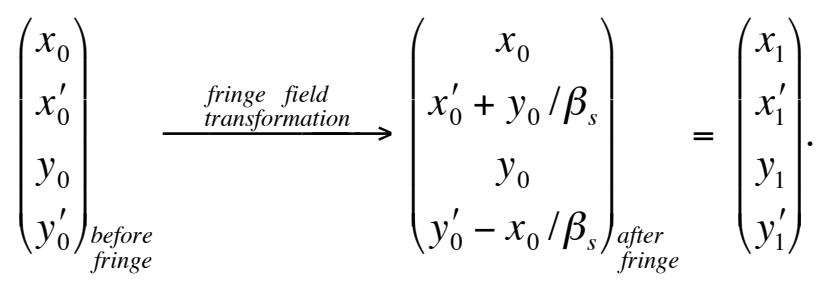

From the definitions above, the drift and cyclotron coordinates are mapped as

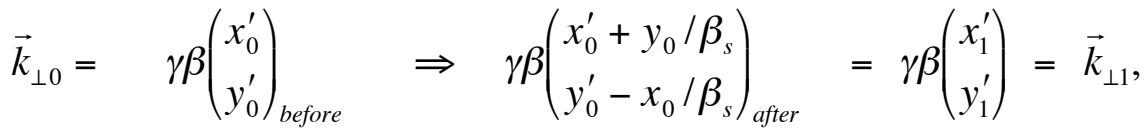

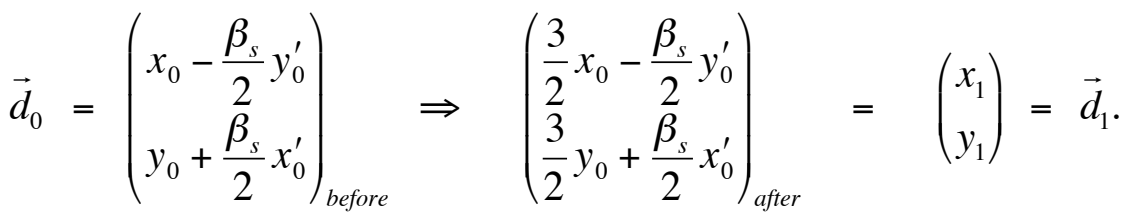


The Cartesian components display coupling between the horizontal and vertical phase spaces after the fringe field transformation. In the laboratory frame, this motion is displayed as a shear in the transverse $(x y)$ plane.

However, the drift and cyclotron modes are known from the previous section to describe independent degrees of freedom. This still remains the case. The drift and cyclotron degrees of freedom are mapped by the fringe field into independent circular modes with oppositely directed angular momenta.

That these are bona fide circular modes in the region following the fringe field may be checked by verifying that particle position is transverse to momentum

$$
\begin{gathered}
\vec{p}_{1}=\left(\prod m c \cdot x \rrbracket \square m c \cdot y \rrbracket\right. \\
\vec{r}_{1} \cdot \vec{p}_{1}=0=\prod m c\left(x_{1} \cdot x \square+y_{1} \cdot y \rrbracket\right. \\
\vec{r}_{1}=\left(x_{1}, y_{1}\right)
\end{gathered}
$$

for each degree of freedom separately. That they are have oppositely-directed angular momenta along the beamline axis $\left(L_{z}\right)$ is verified by direct calculation of $L_{z}=\operatorname{Tmc}\left(x_{1} y \square x \rrbracket \Psi_{1}\right)$ for each independent degree of freedom. For the circular modes derived from the drift and cyclotron motions, the angular momenta are calculated to be

$$
\begin{aligned}
& L_{z}^{\text {drift }}=\square \frac{1}{2} e B_{z}\left(x_{0}^{2}+y_{0}^{2}\right)=\square \frac{1}{2} e B_{z} d_{0}^{2}, \\
& L_{z}^{\text {cyclotron }}=\frac{1}{2} \frac{\left(\prod m c\right)^{2}}{e B_{z}}\left(x y_{\square}^{2}+y_{9}^{\natural}\right)=\frac{1}{2} \frac{k_{\square 0}^{2}}{e B_{z}}(m c)^{2} .
\end{aligned}
$$

From this calculation, we see that the drift and cyclotron degrees of freedom are mapped entirely onto independent circular modes with oppositely-directly angular momenta. The product of these two angular momenta is proportional (up to a sign, and factors of $m c$ ) to the 4-D transverse emittance above. The independent circular mode emittances are merely the magnitudes of their angular momenta (modulo $m c$ ). Hence, the large ratio between the drift and cyclotron emittances from the previous solenoid channel is recovered in the large ratio between the magnitudes of the angular momenta in the circular modes.

\section{Passage Through The SKeW QUADRUPOle BEAM ADAPTER.}

The final step in the process is to pass the beam through the skew quadrupole adapter. We write the coordinates associated with the drift motion as

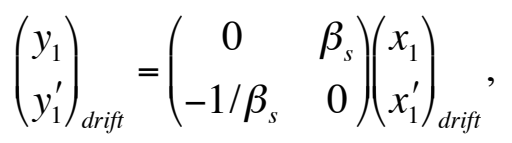

while the coordinates associated with the cyclotron motion are expressible as 


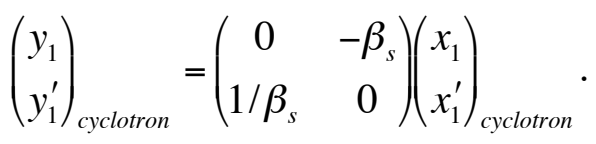

The quadrupole adapter lattice is constructed so that the relative betatron phase advance between the $x$ and $y$ phase spaces is $\pi / 2$, this condition cancels the remnant coupling between the $x$ and $y$ phase spaces. The adapter transforms the two circular modes into the two planes $\left(x, x^{\prime}\right)$ and $\left(y, y^{\prime}\right)$ oriented at $45^{\circ}$ with respect to the quadrupoles' normal planes. Thus, in order to transform the drift component in the solenoid into purely horizontal motion, the adapter has to be rotated by $45^{\circ}$. The resulting linear transformation is then written as

$$
\begin{aligned}
& M_{\substack{\text { skew quad } \\
\text { adapter }}}=\quad M_{\substack{\text { rotation } \\
\text { about } z}}\left(45^{\circ}\right) \circ M_{\substack{\text { quad } \\
\text { lattice }}} \circ M_{\substack{\text { rotation } \\
\text { about } z}}\left(\square 45^{\circ}\right)
\end{aligned}
$$

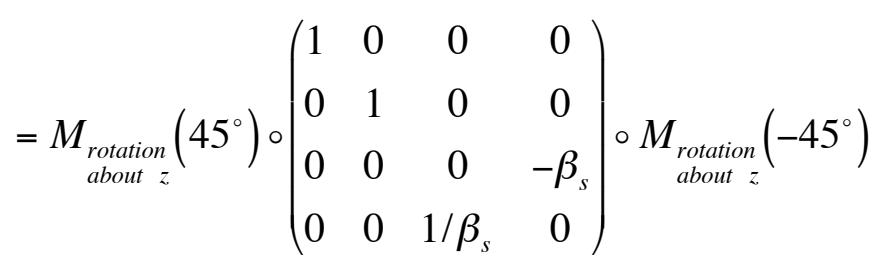

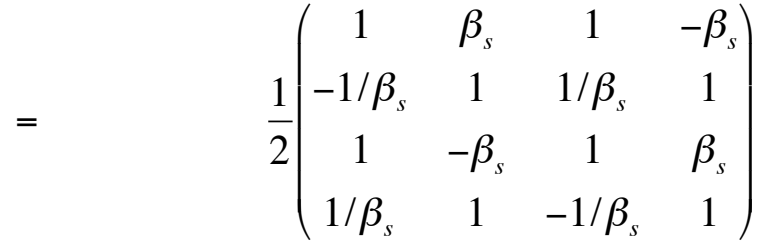

This linear transformation acts upon the independent cyclotron and drift modes, producing purely vertical and horizontal motions

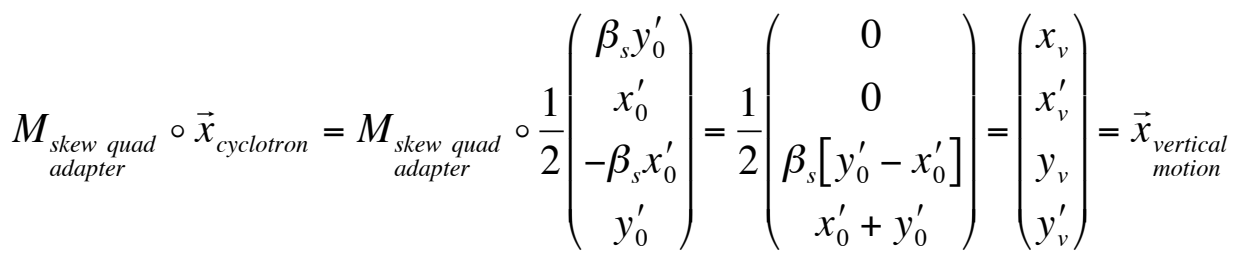

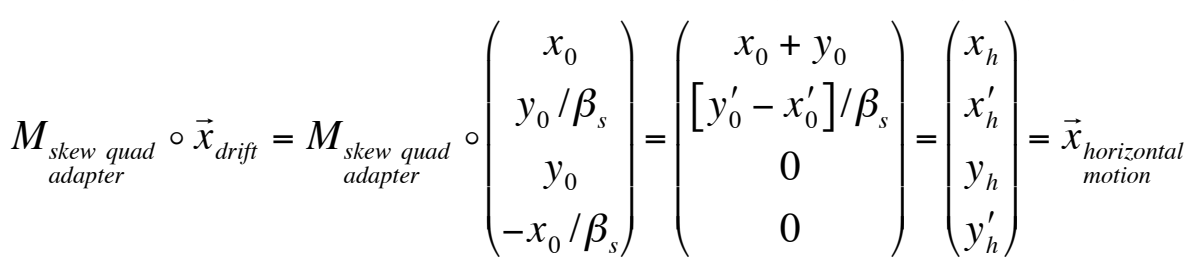

Hence, we see that the cyclotron motion is transformed into purely vertical motion, while the drift motion is transformed to purely horizontal motion. The emittances for these two modes are then 


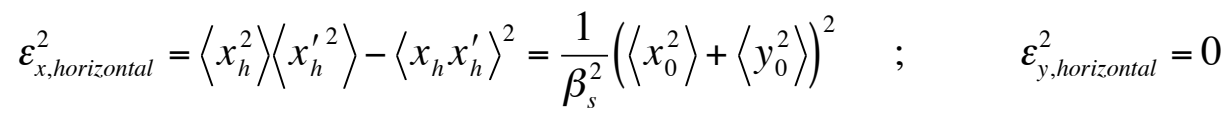

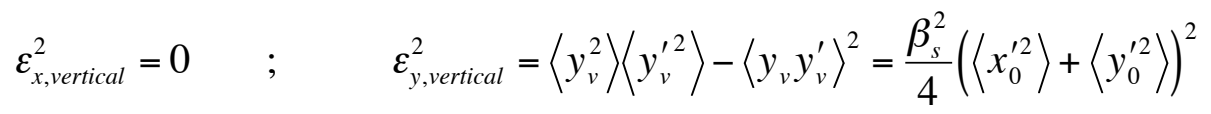

\section{Obtaining Vertical Emittance SMaller Than The Initial Thermal EMitTANCE}

The horizontal and vertical emittances are direct descendants of the emittances of the drift and cyclotron motions (or negative and positive circular mode motions). From the expressions for the horizontal and vertical emittances above, we may write

$$
\begin{aligned}
& \square_{k}=\frac{1}{\square_{s}}\left(\left\langle x_{0}^{2}\right\rangle+\left\langle y_{0}^{2}\right\rangle\right)=\frac{e B_{z}}{2 \square m c}\left(\left\langle x_{0}^{2}\right\rangle+\left\langle y_{0}^{2}\right\rangle\right),
\end{aligned}
$$

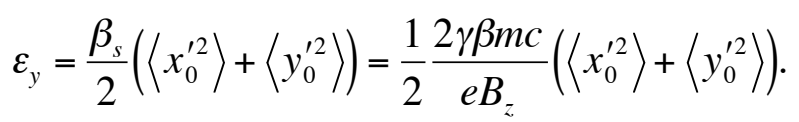

The emittance ratio, then, is [2]

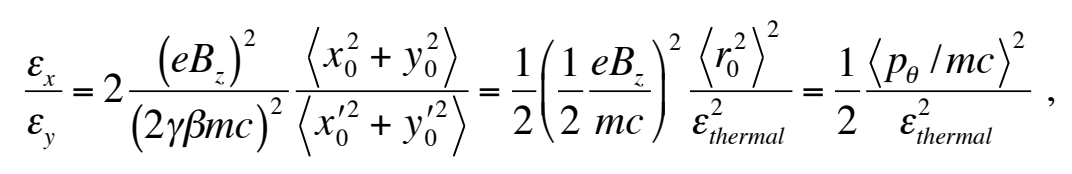

where the thermal emittance is

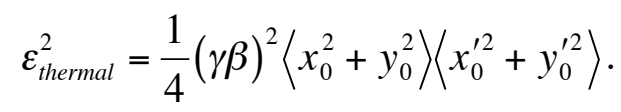

We can identify a condition by which we can obtain a vertical emittance lower than the initial thermal emittance,

$$
\left\langle\frac{p_{\square}}{m c}\right\rangle=\frac{1}{2} \frac{e B_{z}}{m c}\left\langle r_{0}^{2}\right\rangle>\sqrt{2} \square_{\text {hermal }} \text {. }
$$

Since the thermal emittance is proportional to $\sqrt{\left\langle r_{0}^{2}\right\rangle}$, the inequality can be satisfied easily by increasing the spot size at the cathode or the magnetic field at the cathode, or both.

This calculation of the horizontal and vertical emittances at the exit of the flat beam adapter assumes that there is no uncorrelated emittance growth along the beamline. The majority of the emittance growth in the beamline is correlated emittance growth between longitudinal slices of the beam. By employing compensation techniques described previously, the correlated emittance increase may be minimized, and the projected emittance at the adapter entrance can be brought near to the lower limit set by the thermal emittance at the cathode.

The remainder of the emittance growth is primarily attributable to intra-beam scattering from space charge effects. This produces growth in the uncorrelated, thermal spread of transverse 
momenta of the beam electrons. For large drift mode emittance, small changes to the transverse momenta will have little effect. However, the cyclotron mode emittance is proportional to the transverse thermal energy, and it will increase more quickly. The same is true for the horizontal and vertical emittances, respectively, after the adapter. Growth in the uncorrelated (thermal) emittance will impact the vertical emittance more severely than the horizontal, and will drive down the achievable emittance ratio.

\section{Previous Studies of Flat-Beam Transformation - Existence Proof}

Experiments have been conducted at FNPL [5-7] to demonstrate the technique of flat beam production from a photoinjector. We describe the results of those experiments here.

\section{Beam Rotation}

The beam is observed by CCD cameras viewing aluminum-on-glass OTR screens. A sequence of images at and downstream from the first horizontal slit array with nonzero magnetic field on the cathode, but with zero excitation of the adapter quadrupoles, is shown in Figure 8-2. $\mathrm{X} 3$ is located immediately before the entrance to the first skew quadrupole in the adapter lattice, while X4 and X5 are located immediately preceding and following the bunch compressor, respectively. That the beam has angular momentum is demonstrated in the apparent rotation of slit images, and may be measured for comparison with estimates from the theoretical model.

Measurement of the beamlet distributions in the slit images provides a means of determining the vertical emittance (assumed equal to the horizontal since azimuthal symmetry has not been broken), as well as the optical functions of the beam at the location of X3 [8].

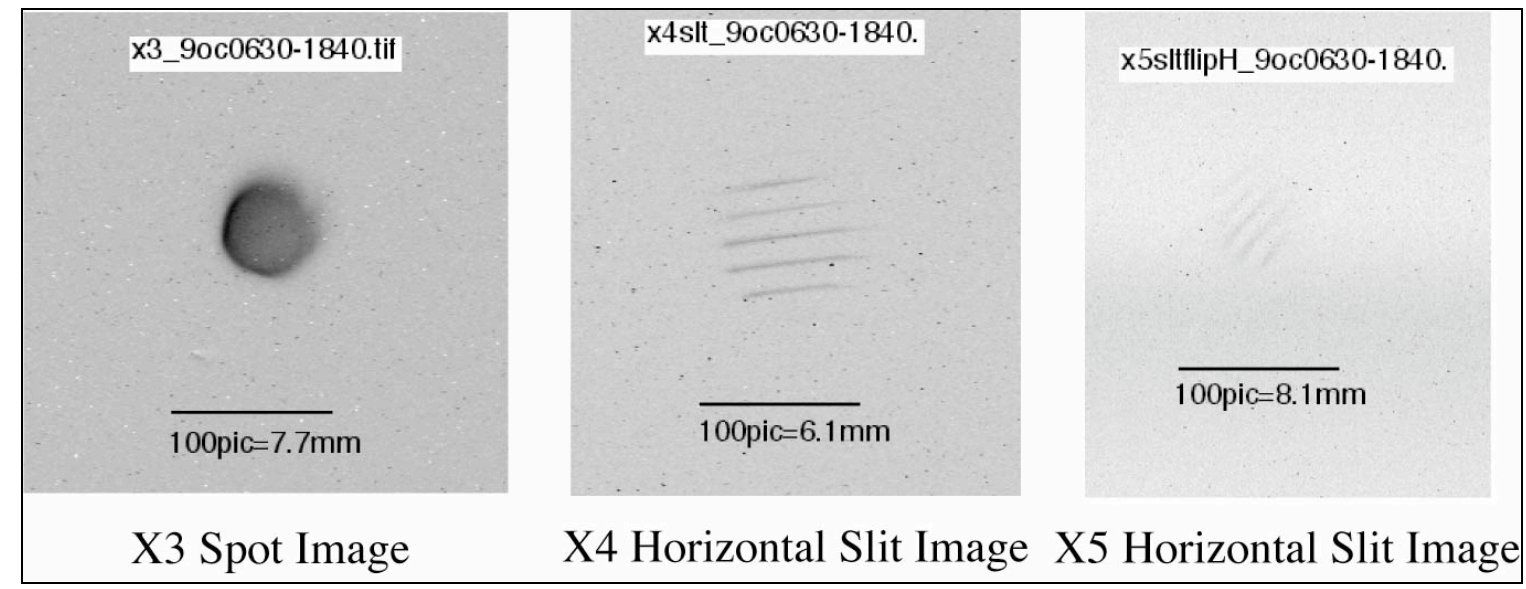

Figure 8-2 Beam spot at slit analyzer and downstream beamlet images showing angular momentum content of beam. 


\section{Flat Beam Emittance}

Flat beams were produced by energizing the skew quadrupoles shown in Figure 8-1 of section 7-Experimental Studies at FNPL. The beam was observed at various locations along the beamline downstream of the skew quadrupole adapter to ensure that the angular momentum was completely removed. At L6, approximately $3 \mathrm{~m}$ downstream from the adapter exit, the beam spot size was measured with an OTR foil. After measuring the spot size, vertical or horizontal analyzer slits were placed into the beamline and the resulting distribution of beamlets was measured at L7 and L8, located approximately $40 \mathrm{~cm}$ and $80 \mathrm{~cm}$, respectively, downstream from the analyzer slits. The spot size and beamlet distributions are shown in Figures 8-3 and 8-4.

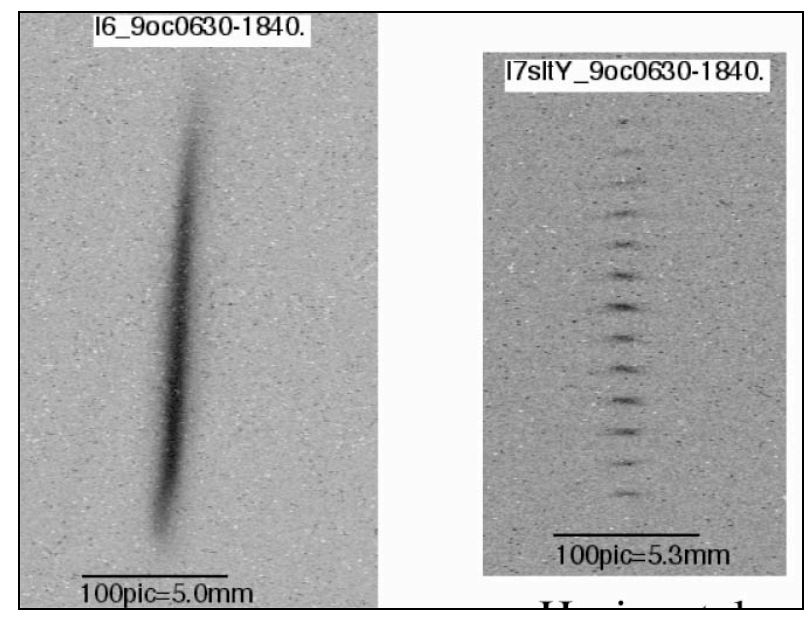

Figure 8-3 Beam spot image downstream from the adapter (left), and beamlet distribution produced by horizontal slits.

In these figures, the beam is 'flat' in the horizontal plane, with the larger emittance plane in the vertical direction. From slit data in this orientation, the measured ratio of emittances is $\sim 50: 45$ $\square \mathrm{m}$ (vertical) by $0.9 \square \mathrm{m}$ (horizontal) for a beam with charge $\sim 1 \mathrm{nC}$. This value is known to be a lower bound on the actual emittance ratio, since the horizontal emittance measurement is resolution limited, as seen in Figure 8-4. The standard deviation of the narrow distribution is comparable to a single pixel of the CCD camera viewing the screen. 


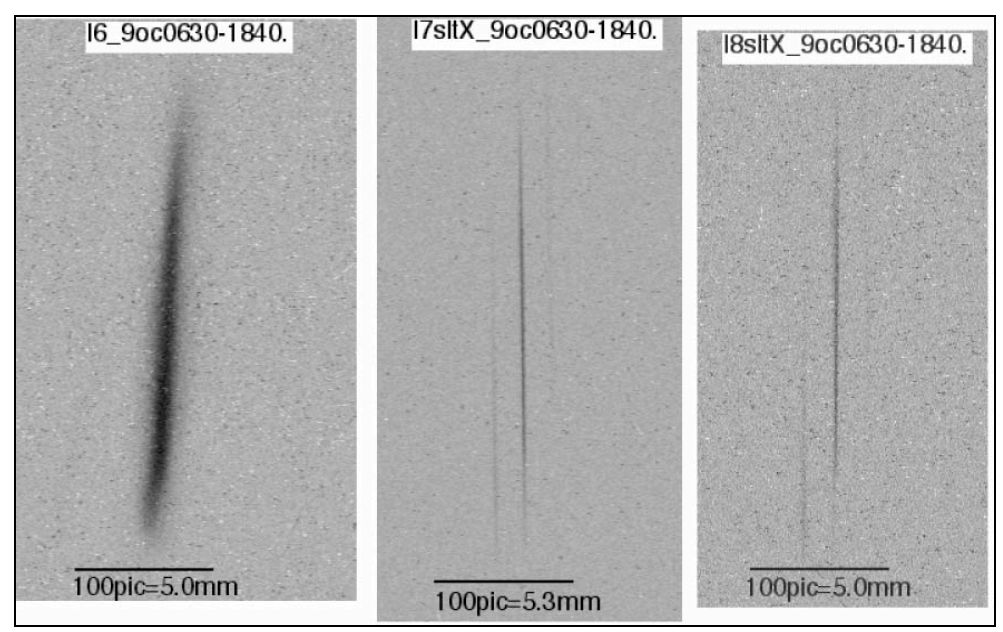

Figure 8-4 Beam spot image downstream from the adapter (left), and beamlet distribution produced by vertical slits (center and right).

\section{Future Work at FNPL}

Work at FNPL will continue to explore means of optimizing the flat beam transformation and to explore the available parameter space.

The influence of space charge effects will be decreased with an increase in the laser pulse length from $10 \mathrm{ps}$ to $30 \mathrm{ps}$. This will primarily affect the space charge induced thermal emittance growth from intra-beam scattering, and thus the growth in cyclotron emittance. However, the effect on the contribution to the projected emittance from correlations between different longitudinal slices must also be studied.

We will carry on parametric studies of emittance compensation and flat beam production. One particular are that will receive more attention in the near future is the sensitivity of the beam emittance to changes in the rf gun gradient and launch phase. We will also study flat beam transforms of higher $(>1 \mathrm{nC})$ charge beams.

The adapter optics and matching will be studied in more detail. The beam optical functions at the adapter entrance will be measured and compared with simulation. We will study via simulation different configurations of the adapter lattice (beyond the three skew quadrupoles) to optimize the flat beam transformation and to minimize the horizontal beam emittance downstream of the adapter.

We will continue working with FNPL personnel to develop diagnostic means of measuring large emittance-ratio beams. The current optical diagnostics are resolution limited, and an increase by a factor of 2-5 is foreseen to measure beams with emittance ratios greater than 50 . We will incorporate the measurement diagnostics into our simulations to more accurately model the experiments. 


\section{Flat BEAM AdAPTER DESign}

Preliminary studies with PARMELA of the integrated 4-cell rf gun with flat beam adapter have been performed to investigate the sensitivities of lattice geometry and space charge effects to emittance of the resulting beam. These on-going studies are being conducted in parallel with HOMDYN studies of emittance compensation described earlier, and will eventually be combined into an integrated description of the complete beam dynamics in the injector.

In the example shown here, we have simulated the beam dynamics in the rf gun for a particular set of parameters, shown in Table 8-1. The rf fields in this case were calculated with SUPERFISH. For the beam dynamics simulations in the region between the cathode and the entrance to the flat beam adapter, where the beamline still maintains azimuthal symmetry, the 2D (r-z) space charge algorithm was employed.

Table 8-1: RF Gun parameters for PARMELA simulation

\begin{tabular}{|l|l|l|}
\hline RF gradient (1st cell / 2nd-4th cells) & $64 / 40$ & $\mathrm{MV} / \mathrm{m}$ \\
Beam energy at RF gun exit & 10.22 & $\mathrm{MeV}$ \\
Solenoid field (peak / cathode) & $1500 / 1032$ & $\mathrm{Gauss}$ \\
Bunch charge & 1 & $\mathrm{nC}$ \\
Pulse length (FWHM) & 20 & $\mathrm{ps}$ \\
Spot size (edge / RMS radius) & $2.4 / 1.7$ & $\mathrm{~mm}$ \\
Thermal emittance (RMS) & 0.7 & $\pi \mathrm{mm}-\mathrm{mrad}$ \\
\hline
\end{tabular}

The solenoid field profile from the cathode plane $(z=0 \mathrm{~cm})$ to the entrance of the flat beam adapter $(z=100 \mathrm{~cm})$ is shown in Figure 8-5. The beam envelope and radial emittance up to the adapter entrance are shown in Figure 8-6.

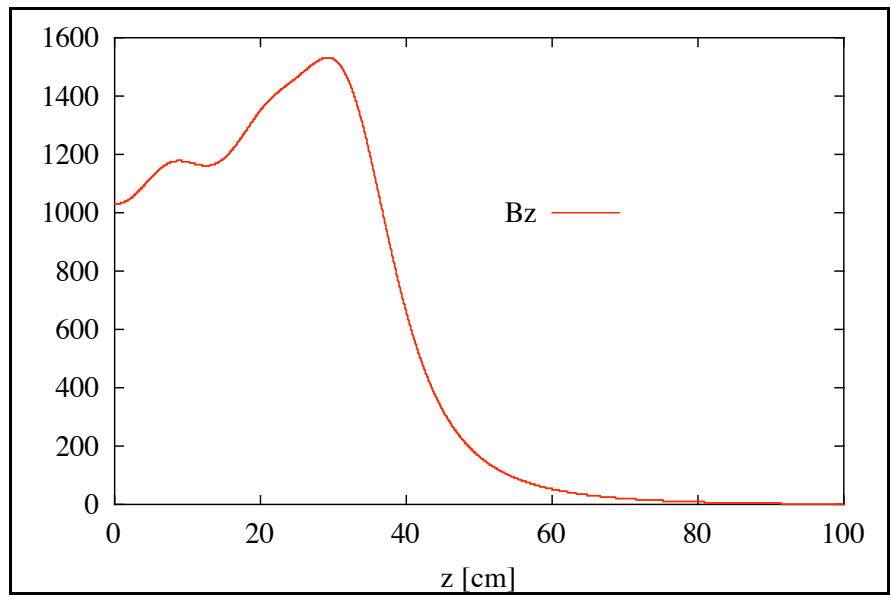

Figure 8-5 Longitudinal distribution of on-axis solenoid field in rf gun 
With a solenoid field value at the cathode of 1032 Gauss and beam edge radius of $2.4 \mathrm{~mm}$, the canonical angular momentum contributes $\left(p_{\square} / 2 m c\right) \sim 44 \pi \mathrm{mm}-\mathrm{mrad}$ to the effective emittance. The theoretical achievable emittance ratio in this configuration is then seen to be $\left(p_{\square} / m c\right)^{2} / 2 Z_{\text {hermal }} \sim 8000$. This simple estimate does not include degradation due to nonlinear space charge and external field influenced emittance growth. Any normal emittance growth experienced between the cathode and the adapter will increase the emittance in the cyclotron degree of freedom and, hence, lower the achievable emittance ratio while raising the lower bound on the achievable vertical emittance.
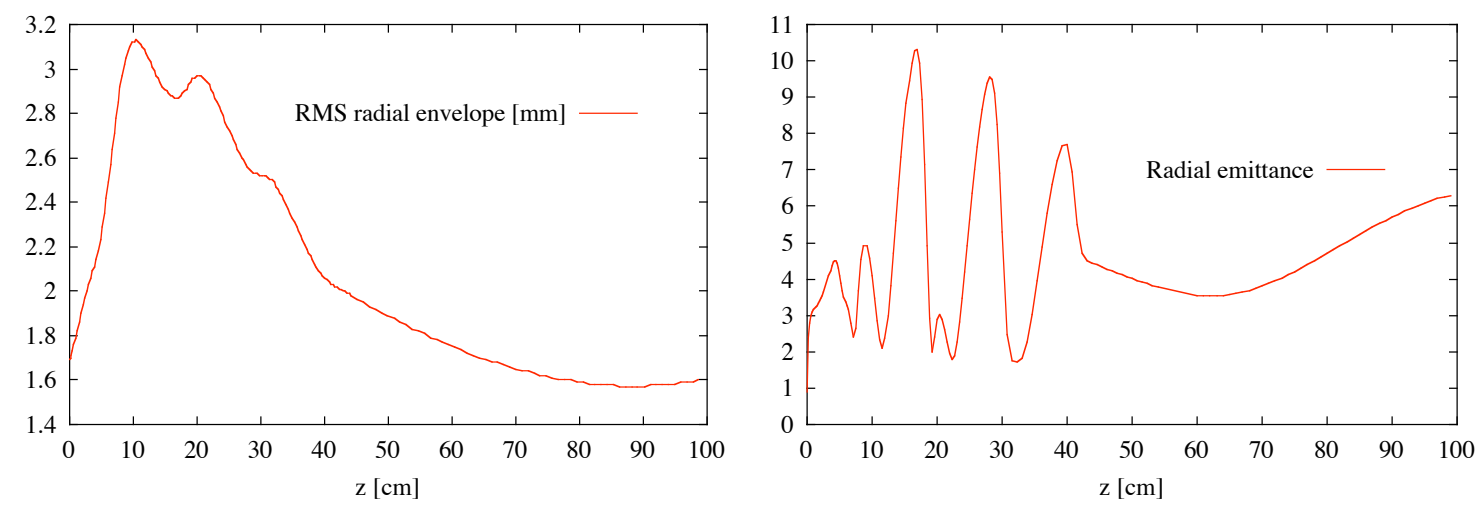

Figure 8-6 RMS beam envelope $(\mathrm{mm})$ and projected $R M S$ radial emittance $(\pi \mathrm{mm}-\mathrm{mrad})$ (PARMELA)

At the entrance to the adapter section, PARMELA calculates the drift mode emittance to be $\sim 70 \pi \mathrm{mm}$-mrad and the cyclotron mode emittance to $\sim 0.097 \pi \mathrm{mm}$-mrad. The adapter skew quadrupole lattice under consideration here is shown in Figure 8-7.

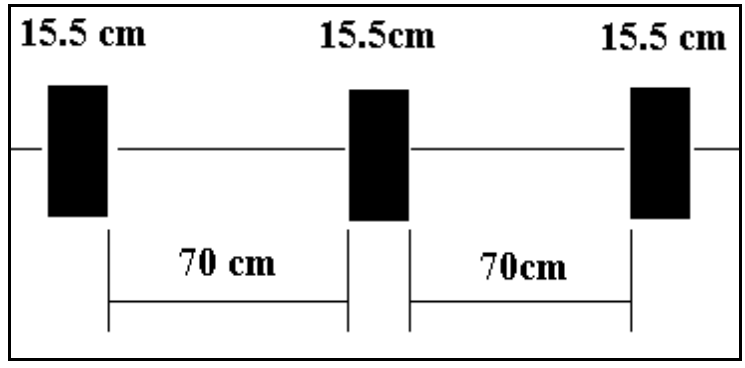

Figure 8-7 Geometry of skew quadrupole adapter beamline.

The hard-edged skew quadrupole gradients were determined to be $-43.75,12.5$, and -5.88 gauss $/ \mathrm{cm}$ for the entrance, middle, and exit quadrupoles, respectively. These gradients were determined by minimizing the vertical emittance at the exit of the adapter section, but without the contribution from space charge effects. Once this operating point was found, 3D space charge contributions were added to subsequent PARMELA runs. The horizontal and vertical emittances calculated are listed in Table 8-2. 
Table 8-2 Calculated emittances from PARMELA in the adapter section

\begin{tabular}{|l|l|l|}
\hline & $\mathrm{Q}(\pi \mathrm{mm}-\mathrm{mrad})$ & $\begin{array}{l}\mathrm{Q},(\pi \mathrm{mm}- \\
\mathrm{mrad})\end{array}$ \\
\hline Adapter entrance & 69.754 & 0.097 \\
\hline Exit: No Space Charge & 69.756 & 0.097 \\
\hline Exit: 3D Space Charge & 69.667 & 0.273 \\
\hline
\end{tabular}

\section{Space charge effects}

From above example, in the absence of space charge effects in the adapter beamline, the circular mode emittances precisely predict the flat beam emittances. However, with the influence of space charge effects included in the calculation, the vertical emittance almost tripled.

The growth in vertical emittance results from two sources: (i) mismatching of the optical functions in the adapter from linear space charge detuning and dispersion; and (ii) emittance growth from mismatching the input beam distribution due to nonlinear space charge forces. Our task, then, is to find the optimal working point in the presence of space charge detuning, to calculate emittance growth from nonlinear space charge forces and dispersion, and to devise means of minimizing the emittance growth.

\section{Non-uniform longitudinal profile - slice effects}

The transverse beam distribution at the exit of the adapter beamline can be analyzed with respect to longitudinal position along the bunch. Space charge and dispersion effects in the upstream portion of the injector redistribute the longitudinal profile from uniform to a more parabolic shape. This is shown in Figure 8-8.

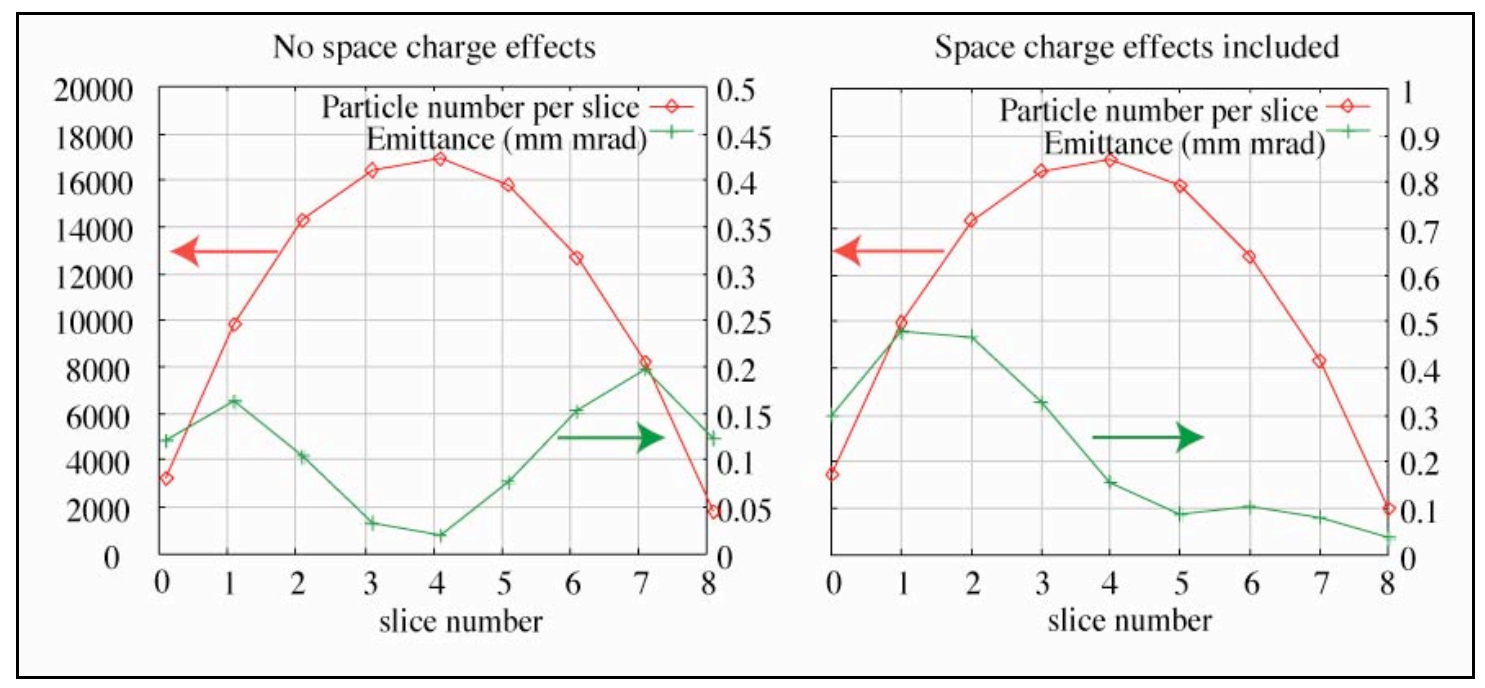

Figure 8-8 Slice population and transverse emittance at the adapter exit. 
In the absence of space charge effects, the symmetry of the slice emittance distribution at the adapter exit follows that of the longitudinal phase space at the adapter entrance. Figure 8-9 shows the influence of space charge effects in the adapter upon the longitudinal phase space. We see that the correlated energy spread approximately doubles in this region.

\section{Matching to the adapter lattice}

The adapter parameters have been tuned to match the center slice of the beam. From the $y-z$ plane view, a dumbbell-shaped distribution is observed. Figure 8-10 shows the head and tail are mismatched with respect to the beam center. They carry residual angular momentum of opposite sign. At the exit of the transformer, the $y-z$ beam distribution shows the matching point has shifted towards the tail (and lower energies). The slice emittance in Figure 8-88.8 shows the head contributing more heavily to the projected emittance.

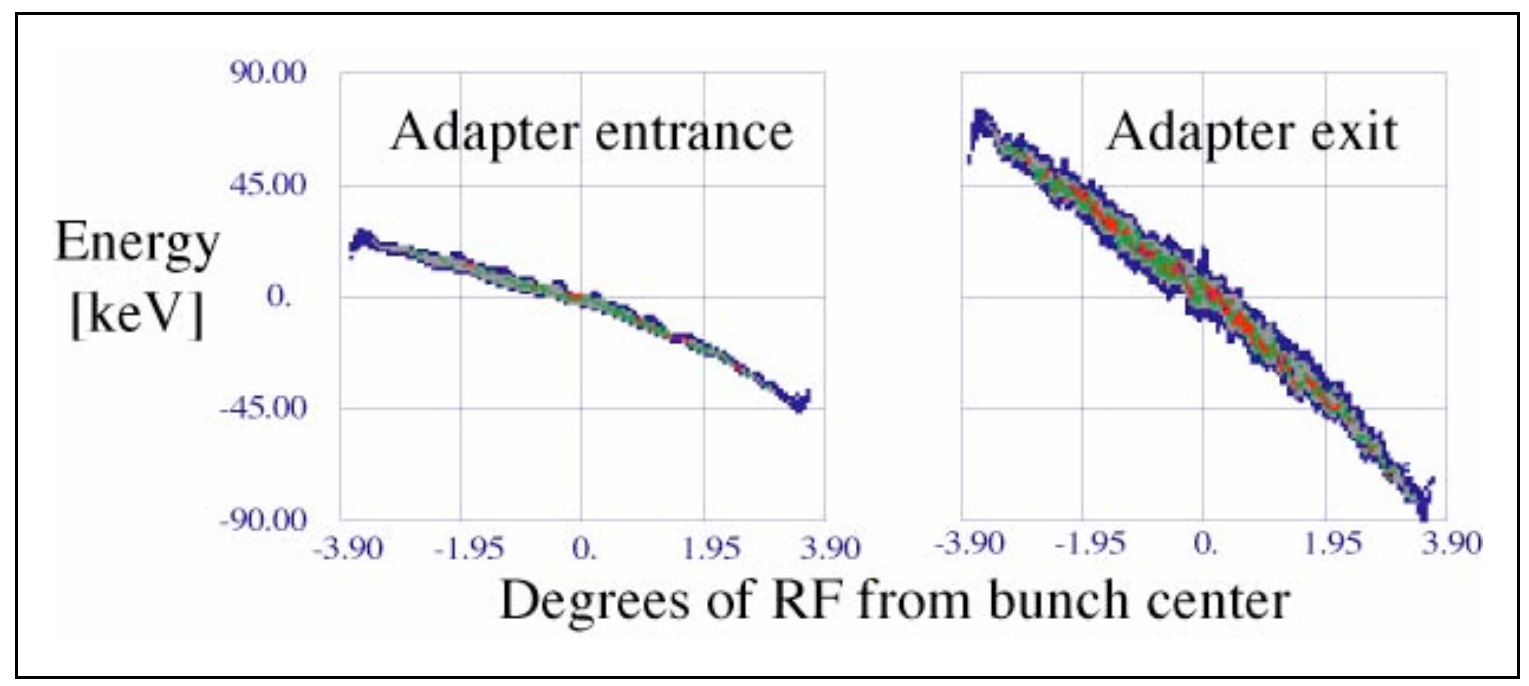

Figure 8-9 Longitudinal phase space at adapter entrance $(z=100 \mathrm{~cm})$ and exit $(z=286.5 \mathrm{~cm})$. 


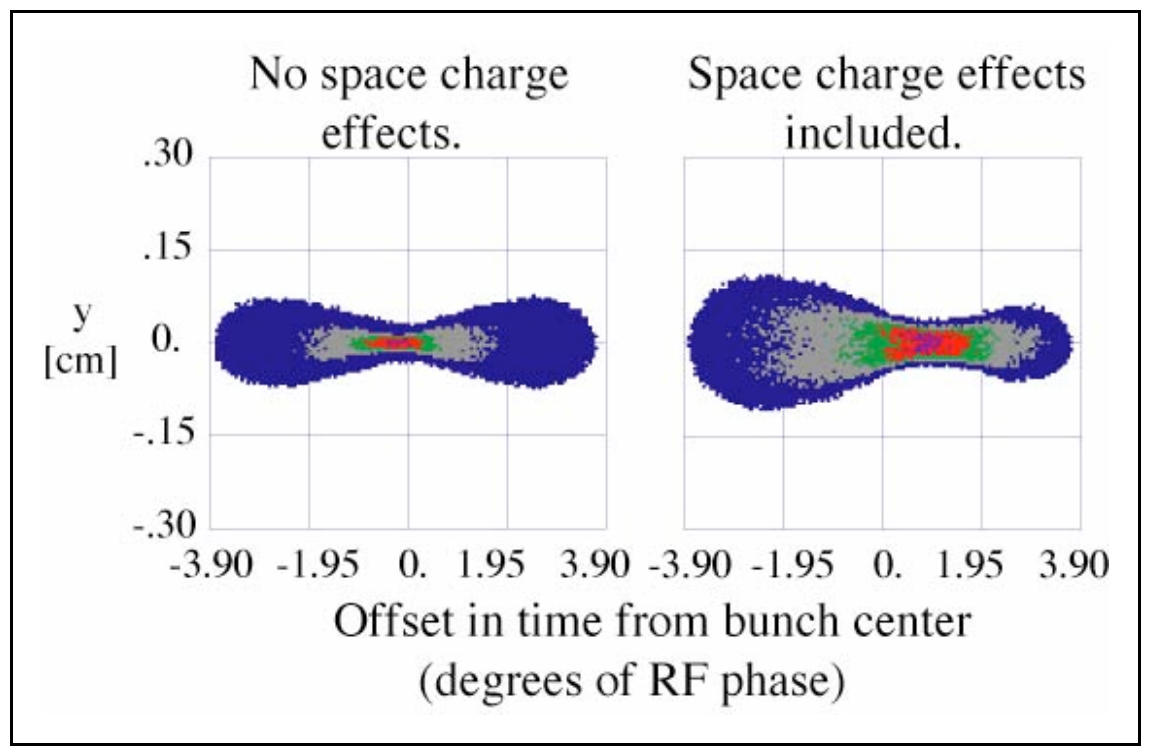

Figure 8-10 The y-z beam distribution at the adapter exit.

\section{Influence of energy mismatch}

To study the effect that a mismatch in the beam energy may have on the adapter transformation without the influence of additional space charge effects, we have simulated the beam dynamics through the adapter by intentionally varying energy at which the adapter is matched. In this way, skew quadrupole gradients have been re-optimized for beam energies varying from $10.22 \mathrm{MeV}$ (the nominal value) to $9.50 \mathrm{MeV}$. The beam (with energy 10.22 $\mathrm{MeV}$ ) is then re-run through this lattice and the output beam distribution is analyzed (see Figure 8-11).

From these plots, we observe that the matching point shifts from the bunch centroid toward the bunch head as the mismatch energy decreases, with a similar behavior to the effect of space charge detuning.

Adding the influence of space charge effects demonstrates this effect more clearly. In Figure 8. 12, we plot the projected vertical emittance of the beam at the adapter exit, both with and without space charge effects, as a function of the mismatch energy. Without space charge effects, the projected emittance rises monotonically with the degree of mismatch, starting from a matched condition at the nominal energy $(10.22 \mathrm{MeV})$. In the case where space charge effects are included, the optimal condition of the lattice corresponds to an energy of $\sim 9.66 \mathrm{MeV}$ (calculated without detuning effects). 


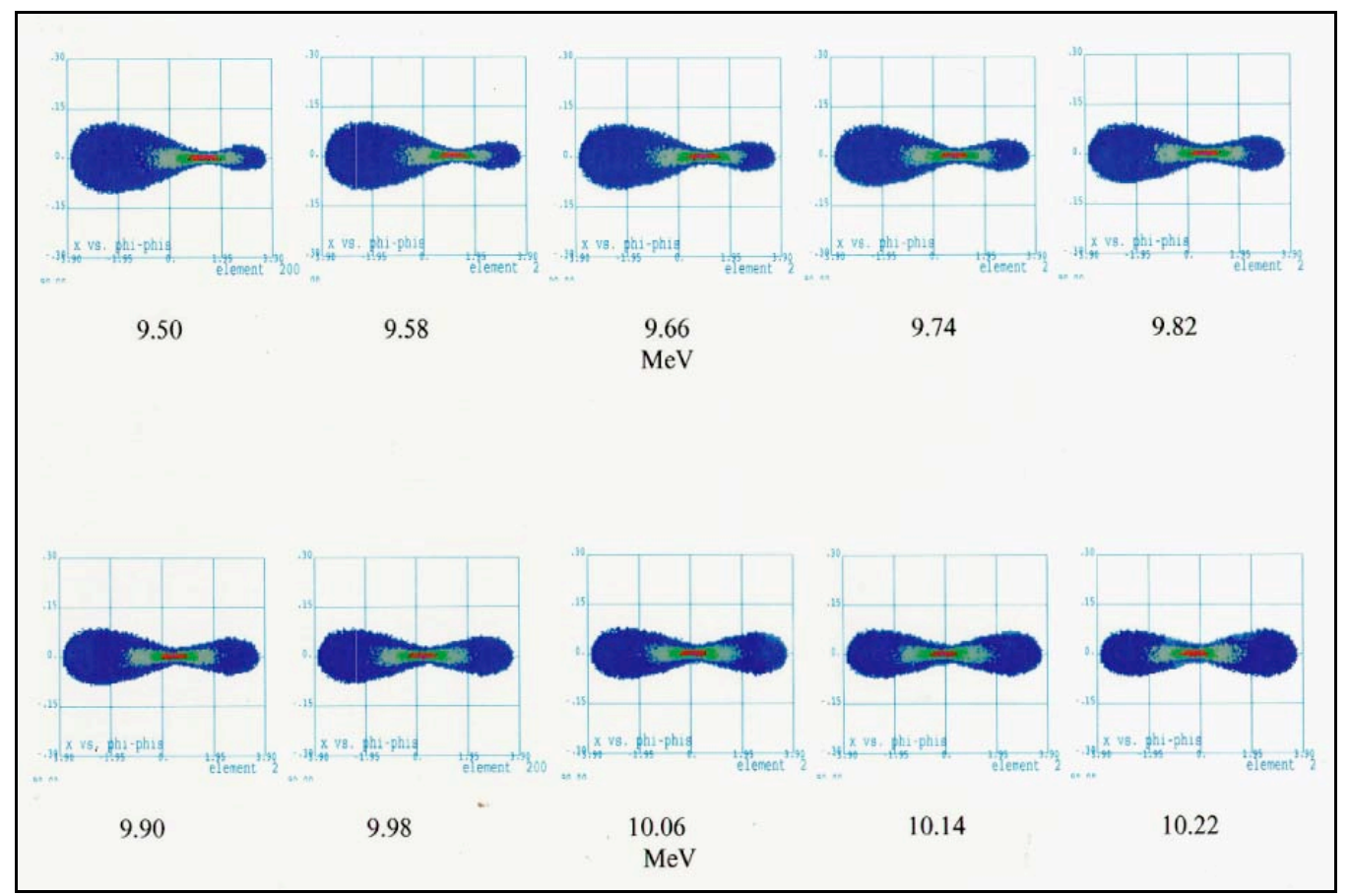

Figure 8-11 Vertical-longitudinal $(y-z)$ beam distribution under energy mismatch conditions (space charge effects neglected).

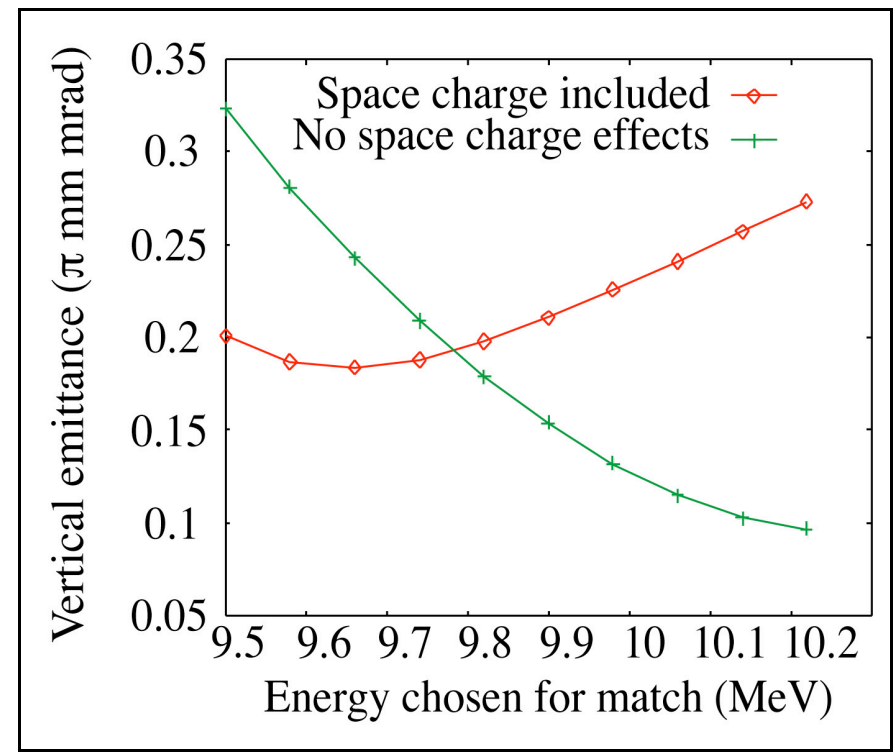

Figure 8-12 Vertical emittance versus adapter mismatch energy. 


\section{Discussion of results and future studies}

The adapter optics beamline and the beam transport simulations shown here do not yet represent an optimized design. The evolution of the radial emittance in the rf gun and following drift section to the adapter entrance has not been optimized for lowest possible emittance. The adapter entrance is, in fact, located near the secondary local minimum of the radial emittance oscillation. The distribution of slice emittances at the adapter entrance (which matches the distribution at the adapter exit in the absence of space charge effects within the adapter; see Figure 8-8) shows the lack of compensation. However, a slightly different tune of the solenoids around the rf gun will place local emittance minimum at the adapter entrance.

We have demonstrated two effects of space charge on the beam transport in the adapter section. First, the effect of linear space charge tune depression alters the matching condition of the beam within the skew quadrupole adapter lattice such that the beam centroid is matched only for higher quadrupole gradients. The effect of beam charge on the betatron phase advances required for the transformation will be studied. Second, the differences in the correlated sectors of the slice emittances resulting in the variation of cyclotron tunes advance for different slices creates a longitudinally varying mismatch condition along the beam. Again, this may be corrected once emittance compensation is employed to it fullest extent.

The energy spread of the beam at the adapter entrance is roughly $\pm 15 \mathrm{keV}$, matching the required specifications. However, this value increases by a factor of $\sim 2$ by the end of the adapter section. Whether this is due to space charge effects only, or if there is an effect due to the coupled transverse beam distributions transiting the skew quadrupole channel will be studied. For a purely longitudinal space charge effect, this correlated energy spread may be corrected by appropriate phasing of the individual rf gun cavities.

We must assess the impact of different optics and models on the emittance development. The fringe-field at the exit of the solenoid region has not been optimized. Implementing iron field clamps at the exit will cause the fringe to decay more rapidly without affecting the cyclotron tune. The decay of the fringe is responsible, however, for preparing the beam to be injected into the adapter. A more detailed study of the dynamics within the fringe field region needs to be performed and the impact on the emittances clarified. Likewise, the skew quadrupoles used in the adapter will have very significant fringe fields, so that simulation of the beam dynamics within the adapter will need to include the effect of pseudo-multipoles.

\section{REFERENCES}

[1] A.V. Burov and V.V. Danilov, 'An Insertion to Eliminate Horizontal Temperature of High Energy Electron Beam,’ FERMILAB-TM-2043, 1998.

[2] A. Burov, et al., 'Optical Principles of Beam Transport for Relativistic Cooling,' FERMILAB-Pub-00/100-T, 2000.

[3] A. Burov, et al., 'Circular Modes, Beam Adapters and their Applications in Beam Optics', FERMILAB-Pub-01/060-T, 2001.

[4] R. Brinkmann, et. al., 'A Flat Beam Electron Source for Linear Colliders,' TESLA 99-09, DESY-Hamburg, 1999. 
[5] I. Bohnet, et al., 'The Flat Beam Experiment at the FNAL Photoinjector,' Proceedings of the XX International Linac Conference, Monterey, 2000.

[6] D. Edwards, et al., 'Status of Flat Electron Beam Production', Proceeding of the 2001 Particle Accelerator Conference, (IEEE, New York), 2001.

[7] E. Thrane, et al., 'Photoinjector Production of a Flat Electron Beam,' to appear in Proceedings of the XXI International Linac Conference, Gyeongyu, Korea, 2002.

[8] S.G. Anderson, et al., 'Space-charge effects in high brightness electron beam emittance measurements,' Physical Review Special Topics - Accelerators and Beams, 5, 014201 (2002). 


\section{SUPERCONDUCTING RF LINACS}

\section{Choice of RF Technology}

The baseline recirculating linac design requires acceleration through four passes in a $600 \mathrm{MeV}$ linac up to a final energy of $2.5 \mathrm{GeV}$. A $1 \mathrm{nC}$ bunch is launched from the $\mathrm{rf}$ photocathode gun at a $10 \mathrm{kHz}$ rate, and passes through the linac four times to give an average current of $40 \mu \mathrm{A}$ in the main linac.

Tremendous progress has been made in both superconducting and normal conducting rf linac technology in recent years, mainly resulting from research on linear collider projects. The proposed linac design should take advantage of these developments.

Research on superconducting structures for the TeV-Energy Superconducting Linear Accelerator (TESLA) project has greatly advanced the technology [1]. Accelerating gradients of $42 \mathrm{MV} / \mathrm{m}$ have been achieved in an electro-polished seamless single cell $\mathrm{Nb}$ cavity at $1.3 \mathrm{GHz}$, and 9-cell TESLA cavities have been tested at gradients exceeding $25 \mathrm{MV} / \mathrm{m}$ and with unloaded quality factors $Q_{0}$ over $10^{10}$. More recently an electro-polished 9-cell $\mathrm{Nb}$ cavity has reached a record gradient of $32 \mathrm{MV} / \mathrm{m}[2]$.

The development of copper structures has a long history and the technology is relatively mature. In recent years the Next Linear Collider (NLC) project has led the field in research on room temperature copper rf structures for high-gradient linacs, and gradients of over $50 \mathrm{MV} / \mathrm{m}$ have been demonstrated at $11.4 \mathrm{GHz}$ although $\mathrm{rf}$ breakdown was observed around $70 \mathrm{MV} / \mathrm{m}$ in some structures [3]. The SLAC S-band $(2.856 \mathrm{GHz})$ linac has been running successfully at $\sim 20 \mathrm{MV} / \mathrm{m}$ for decades.

In principle, both superconducting and normal warm copper structures are capable of providing the required accelerating gradient for the re-circulating linac although they each raise different technical challenges. Table 9-1 summarizes parameters for several linac options assuming using either superconducting (SC) or normal conducting (NC) rf technologies.

For the NC rf approach, SLAC S-band and X-band traveling wave (TW) linac parameters are used, assuming a $3 \square$ filling time without flat top for all the calculations. The NC L-band linac calculations were scaled from the SLAC S-band linac parameters. For the SC linac, parameters for the TESLA Test Facility (TTF) cryomodule are used.

The large quality factors for the SC structures $\left(Q_{0} \sim 10^{10}\right)$ result in a very long filling time $(>1$ $\mathrm{ms}$ at $1.3 \mathrm{GHz}$ ), and the SC linac must be operated in continuous wave (CW) mode for the required $10 \mathrm{kHz}$ bunch repetition rate (100 $\mu$ s time interval). The resultant power dissipation due to $\mathrm{rf}$ currents on the cavity inner surfaces increases significantly over the TESLA design parameters, and implications will be discussed in Chapter 13-Cryogenics. A cryogenic system of approximately $3.5 \mathrm{MW}$ capacity is required for operations of the whole facility at $20 \mathrm{MV} / \mathrm{m}$.

Conventional room temperature linacs can be operated in pulsed mode at $10 \mathrm{kHz}$, but require $\sim 10^{3}$ increase in peak rf power over the SC linac. Operating at high duty factor of approximately $8 \%$, and dissipating $\sim 1 \mathrm{MW} / \mathrm{m}$ average rf power, such linacs are technically very demanding and are not known to exist for such applications. 
To preserve beam emittance, the influence of wakefields in the linac must be minimized. Wakefield amplitude scales with frequency as $W_{L} \mu f^{2}$ and $W_{T} \mu f^{3}$, where $W_{L}$ and $W_{T}$ denote longitudinal and transverse wakefield, respectively. The lower frequency linac thus has a significant advantage in terms of beam dynamics.

Even at the same resonant frequency, normal conducting cavities usually have optimized geometry to provide much larger $R / Q$ values compared to super-conducting cavities, in order to improve the efficiency of the structure (maximized accelerating voltage per unit input power, and minimized wall losses). The large $R / Q$ leads to increased wakefield amplitude and higher loss factors. For superconducting cavities, however, the cavity geometry is optimized to eliminate multipacting and field emission, since quality factor $Q$ may be many orders of magnitude higher than for copper cavities cavities, and $R / Q$ is not as significant a concern.

Table 9-1 Comparisons between superconducting (SC) and normal conducting (NC) rf systems for a $600 \mathrm{MeV}$ linac

\begin{tabular}{|l|c|c|c|c|}
\hline \multicolumn{1}{|c|}{ Parameter } & NC S-Band & NC L-Band & NC X-Band & SC L-Band \\
\hline Frequency $[\mathrm{GHz}]$ & 2.856 & 1.3 & 11.4 & 1.3 \\
\hline Phase Advance & $2 \square / 3(\mathrm{TW})$ & $2 \square / 3(\mathrm{TW})$ & $2 \square / 3(\mathrm{TW})$ & $\square(\mathrm{SW})$ \\
\hline Shunt Impedance $[\mathrm{M} \square / \mathrm{m}]$ & 50 & 34 & 80 & $\sim 10^{7}$ \\
\hline Accelerating Gradient $[\mathrm{MV} / \mathrm{m}]$ & 20 & 20 & 50 & 20 \\
\hline Pulse Length $[\square \mathrm{s}]$ & 2.5 & 8.1 & 0.3 & $\mathrm{CW}$ \\
\hline Quality Factor (unloaded) & 15,000 & 22,000 & 7,000 & $10^{10}$ \\
\hline Quality Factor (loaded) & 7,500 & 11,000 & 3,500 & $2.6 \times 10^{7}$ \\
\hline Linac Length $($ active) $[\mathrm{m}]$ & 30 & 30 & 12 & 33 \\
\hline Filling time $\square=(2 \mathrm{Q} / \mathrm{L})[\square \mathrm{s}]$ & 8.3 & 2.7 & 0.098 & $\sim 3180$ \\
\hline Repetition Rate $[\mathrm{Hz}]$ & 10,000 & 10,000 & 10,000 & $\mathrm{CW}$ \\
\hline Duty Factor & $2.5 \%$ & $8.1 \%$ & $0.3 \%$ & $\mathrm{CW}$ \\
\hline Total Peak RF Power $[\mathrm{MW}]$ & 240 & 354 & 375 & 0.288 \\
\hline Total Average RF Power $[\mathrm{MW}]$ & 6 & 29 & 1.13 & 0.288 \\
\hline
\end{tabular}

Based on these considerations superconducting rf linac technology has been chosen for the recirculating linac, and our design is based on TESLA 1.3 GHz cavities and cryomodules [1].

\section{Linac Design}

The linac design is based on superconducting rf technology developed for the TESLA project [1]. We propose to use modules similar to those designed for the TESLA-FEL injector to take advantage of the developments made by the TESLA collaboration in obtaining high gradient accelerator cavities, and to avoid an expensive and time-consuming R\&D program. The TESLA cavity parameters are well suited to our requirements, and many complex issues including higher-order mode damping, suppression of multipacting, cavity materials preparation and fabrication, input power coupler design, have been studied and solutions explored. 
We also follow developments in the Continuous Electron Beam Accelerator Facility (CEBAF) upgrade project at Jefferson Laboratory. The CEBAF upgrade module incorporates 7cell cavities at $1.5 \mathrm{GHz}$ and aims at $20 \mathrm{MV} / \mathrm{m}$. This design could prove to be a viable choice in the future.

Figure 9-1 shows the cross-section schematic view of a TESLA 9-cell superconducting rf (scrf) cavity [1]. Eight such cavities are integrated with cryogenics and other systems into a TESLA FEL injector linac cryomodule, with cross-section (end view) shown in Figure 9-2. Each of these cryomodules is approximately $12 \mathrm{~m}$ long, and four such cryomodules are required to achieve a total energy of $\geq 2400 \mathrm{GeV}$, i.e. $\geq 600 \mathrm{MeV}$ per pass. We will operate slightly off-crest of the rf field maximum and the design considerations here are for a peak accelerating gradient of $20 \mathrm{MV} / \mathrm{m}$. Operating at the TESLA gradient of $23 \mathrm{MV} / \mathrm{m}$ would allow a final beam energy of $\sim 3 \mathrm{GeV}$, but the lower energy gain provides a design margin and reduces the power dissipation in the cryostat.

The injector linac consists of an additional cryomodule, with reduced gradient of $15 \mathrm{MV} / \mathrm{m}$ providing a total energy gain of $110 \mathrm{MeV}$.

The superconducting cavities have a high intrinsic quality factor $\left(Q_{0} \sim 10^{10}\right)$ resulting in a cavity time constant of about 2.4 seconds. The effective time constant of an SC cavity in the circuit environment is two to three orders of magnitude lower since determined by the loaded $Q$, the choice of which is discussed below. Typically, power is applied to standing wave cavities about three time constants before the beam enters the structure. This allows time for energy to build up in the cavity so that the required field can be developed. Since we want to operate at bunch rates of $10 \mathrm{kHz}$ and greater, the resulting repetition time of $100 \square \mathrm{s}$ is much too short to change the field in the cavities commensurately.

The linac rf system must therefore be operated in continuous wave $(\mathrm{CW})$ mode. This, of course, increases the average power requirements of the system, both in terms of the rf drive power and the cryogenic system power, which is needed to absorb the wall losses in the cavities.

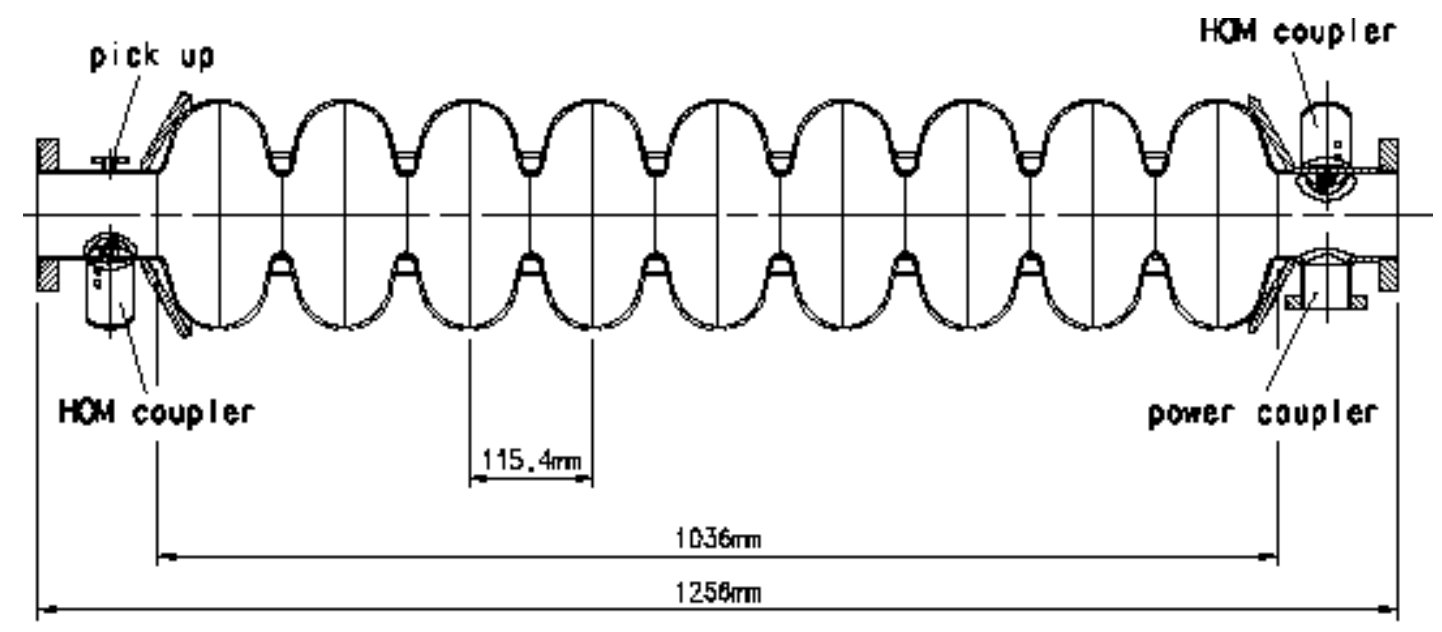

Figure 9-1 TESLA 9-cell superconducting $1.3 \mathrm{GHz}$ cavity, longitudinal cross-section. 


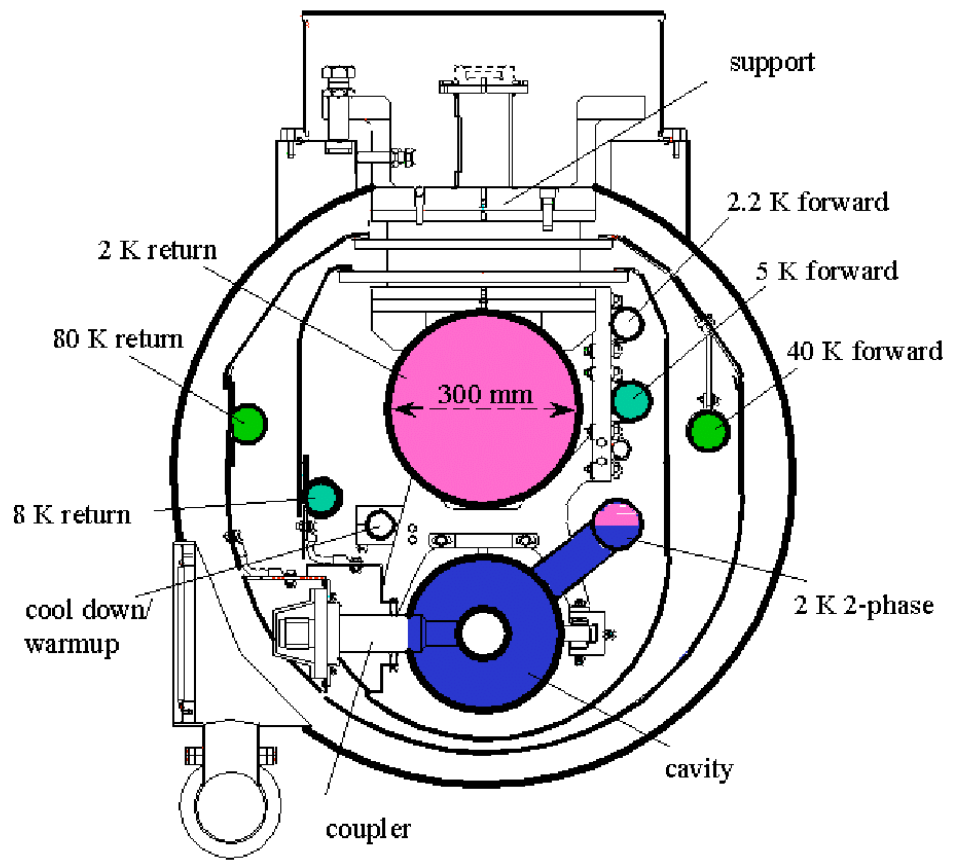

Figure 9-2 TESLA cryomodule end view cross-section

There are a total of 32 cavities in the baseline design $600 \mathrm{MeV}$ main linac, therefore the accelerating voltage for on-crest acceleration is $600 / 32=18.75 \mathrm{MV}$ per cavity. Operation offcrest by as much as $10^{\circ}$ brings this to $\sim 19 \mathrm{MV}$. Together with a nominal length of $1.038 \mathrm{~m}$ per cavity the nominal gradient is $18.34 \mathrm{MV} / \mathrm{m}$. To provide some safety margin these figures are rounded respectively to $20 \mathrm{MV}$ per cavity and $20 \mathrm{MV} / \mathrm{m}$ cavity gradient.

\section{Tuning Variations of the TESLA Cavity}

The basic parameters of the TESLA cavity are

$$
\begin{aligned}
& f_{\mathrm{r} f}=1300 \mathrm{MHz}, \\
& Q_{0}=1 * 10^{10}, \\
& \left.R / Q=518 \square \text { (equivalent circuit definition } \mathrm{R} / \mathrm{Q}=\mathrm{V}^{2} /(2 \square \mathrm{U})\right)
\end{aligned}
$$

The intrinsic 3-dB bandwidth of the TESLA cavity is then

$$
B W_{0}=\frac{f_{r f}}{Q_{0}} \square \frac{1300 \square 10^{6}}{10^{10}} \square 0.13 \mathrm{~Hz}
$$

The big problem for the use of superconducting cavities is the fact that systematic as well as random tuning errors are orders of magnitude larger than this intrinsic bandwidth. 
An example for the systematic part is by the detuning by the radiation pressure forces or Lorentz force detuning, given by [4]

$$
\frac{\partial f_{r}}{\partial U^{2}} \square 0.9 \frac{H z}{\left(\frac{M V}{m}\right)^{2}}
$$

This effect is as large as $360 \mathrm{~Hz}$ for a gradient of $20 \mathrm{MV} / \mathrm{m}$. It is of great concern for pulsed cavities, but can be easily corrected in the present application where the field is continuous.

More serious are the random tuning deviations. Figures 9-3 through 9-5 below show measurements taken at a TTF cryomodule [4].

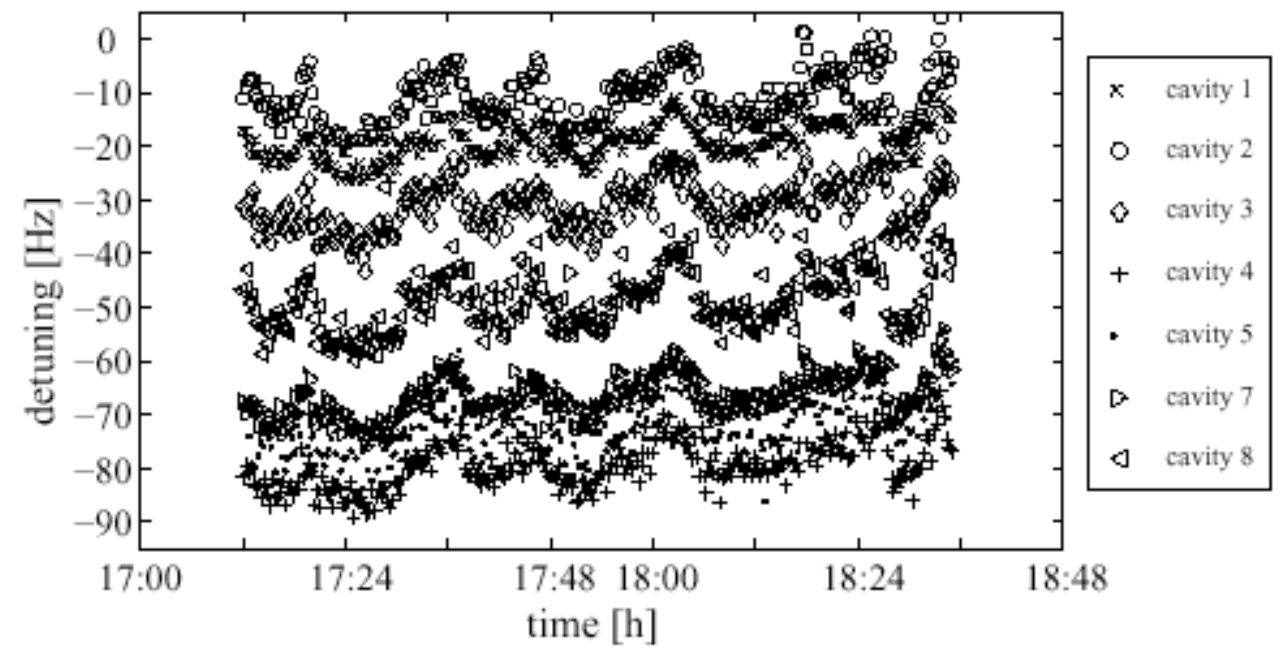

Figure 9-3 Longterm variation of the resonant frequency of the eight cavities in a TTF cryomodule.

The random tuning perturbations fall in two categories:

A. Relatively slow perturbations, with periodic intervals in the minute range. The detuning of all eight cavities is highly correlated; this hints at random variations of the helium pressure as the common cause. The cavity resonant frequency and the pressure variations are related by the factor

$$
\frac{\partial f_{r}}{\partial p} \square 20 \frac{H z}{m b a r}
$$

The behavior of cavity \#1 is shown in detail in Figure 9-4. 

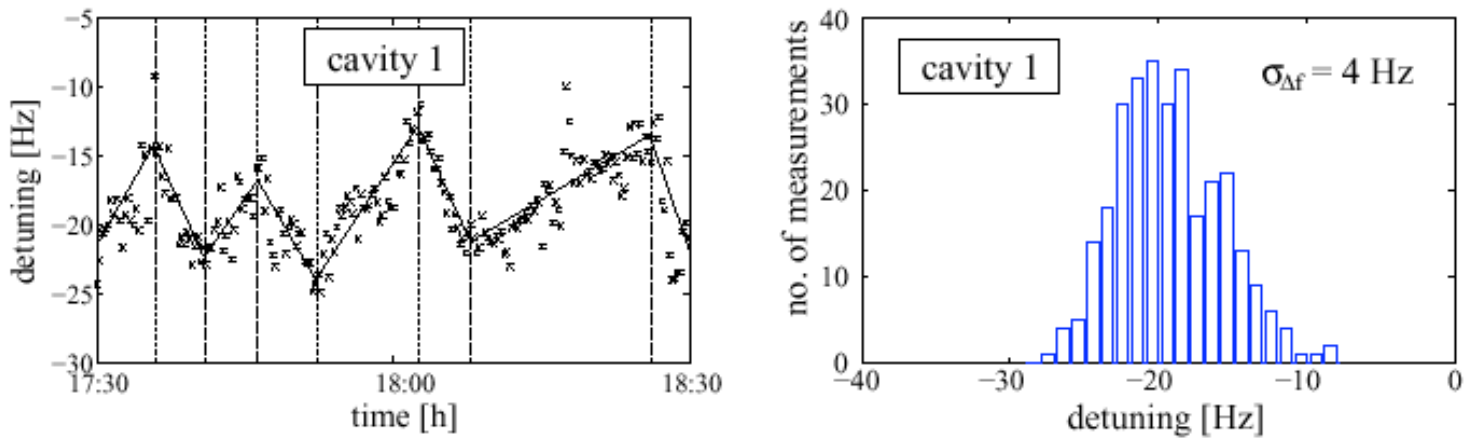

Figure 9-4 Resonant frequency variation of cavity \#1 (left), and corresponding histogram (right)

The periodic tuning variations are shown on the left, together with the assumed contribution of the helium pressure changes which are represented by straight lines. A histogram of the uncorrected raw data points is given on the right, showing a standard deviation of $\square_{\square \mathrm{f}}=4 \mathrm{~Hz}$ and a mean tuning error of $19 \mathrm{~Hz}$

B. Fast perturbations due to microphonics in the acoustical frequency range, caused by mechanical stimuli (pumps, turbulence in the helium flow etc). The response is shaped by structural resonances and directional sensitivities of the cavities. Figure 9-5 shows synthesized histograms that consider only these small "residual microphonics" around the triangular lines in Figure 4 (left). It is assumed that the slow variations have been eliminated by a mechanical tuner, with control bandwidth $\sim 1 \mathrm{~Hz}$.

The fact that these histograms show a Gaussian-like distribution is a strong hint that they are created by a multitude of uncorrelated resonances, since single resonances would lead to peaks at the tails of the distribution [4]. The results are summarized in Table 9-2.

Table 9-2 Microphonic levels of the cavities in the TTF cryomodule; total rms error, and rms error after elimination of low frequency components

\begin{tabular}{|c|c|c|c|c|c|}
\hline $\begin{array}{c}\text { Cavity } \\
\text { no. }\end{array}$ & $\begin{array}{c}\square_{\square \mathrm{ff}}(\mathrm{rms}) \\
\text { raw data }\end{array}$ & $\begin{array}{c}\square_{\square \mathrm{f}}(\mathrm{rms}) \\
\text { slow error eliminated }\end{array}$ & $\begin{array}{c}\text { Cavity } \\
\text { no. }\end{array}$ & $\begin{array}{c}\square_{\square \mathrm{f}}(\mathrm{rms}) \\
\text { raw data }\end{array}$ & $\begin{array}{c}\square_{\square \mathrm{f}}(\mathrm{rms}) \\
\text { slow error eliminated }\end{array}$ \\
\hline 1 & $4 \mathrm{~Hz}$ & $2 \mathrm{~Hz}$ & 5 & $9 \mathrm{~Hz}$ & $7 \mathrm{~Hz}$ \\
\hline 2 & $5.5 \mathrm{~Hz}$ & $4 \mathrm{~Hz}$ & 6 & $($ detuned $)$ & $($ detuned $)$ \\
\hline 3 & $5 \mathrm{~Hz}$ & $3 \mathrm{~Hz}$ & 7 & $4.5 \mathrm{~Hz}$ & $3 \mathrm{~Hz}$ \\
\hline 4 & $7 \mathrm{~Hz}$ & $6 \mathrm{~Hz}$ & 8 & $6.5 \mathrm{~Hz}$ & $5 \mathrm{~Hz}$ \\
\hline
\end{tabular}

As a basis for the design of the rf system, we take as a reference the performance of cavity \# 5 which shows the largest residual microphonics of $\square_{\square \mathrm{f}}=7 \mathrm{~Hz}$ (corrected for slow variations). The largest fast tuning variation is expected $3 \cdot \square_{\square \mathrm{f}} \sim 20 \mathrm{~Hz}$. It appears realistic to assume that the slow tuning offsets can be reduced to $\sim 5 \mathrm{~Hz}$, and it is further assumed that these two components add linearly. The total tuning excursion to be taken into account is therefore about $25 \mathrm{~Hz}$. 
It may be tacitly assumed that through additional R\&D future generations of superconducting cavities will show equal or superior microphonics characteristics than the units considered here.
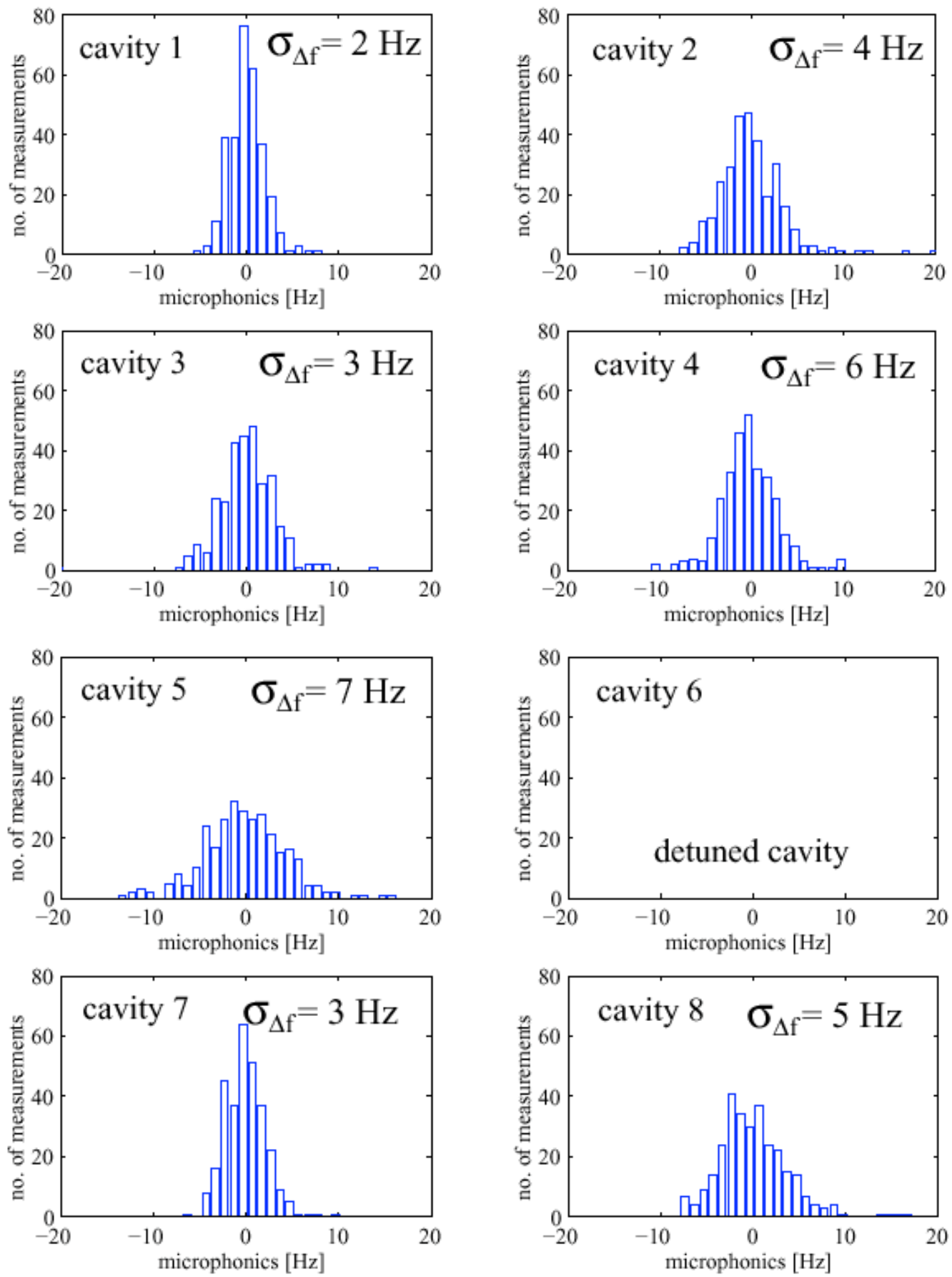

Figure 9-5 Microphonics in the TTF module (helium pressure variations compensated by hypothetical tuner) 


\section{Choice of the Cavity Coupling Factor}

The coupling between the SC cavity and the rf generator determines the overall system bandwidth and is therefore a key factor for the ability of the rf system to cope with cavity tuning variations. The amount of coupling is usually expressed in terms of the coupling factor $\square$, defined as

$$
\square=\frac{r_{\text {shunt }} / k^{2}}{Z}
$$

where $Z$ is the characteristic impedance of the input power feed line, $r_{\text {shunt }} / k^{2}$ is the cavity shunt impedance (circuit convention) transformed to the input line by the voltage transmission factor $\mathrm{k}$ of the coupler.

In other words, $\square$ is the mismatch factor or voltage standing wave ratio (VSWR) of the cavity input port with respect to the generator impedance $Z$, and $Z=1$ would lead to a perfect match of the bare cavity exempt of beam loading.

Klystrons have to be protected against load mismatch by a circulator or isolator, which in turn provides an almost ideal generator output impedance $Z$ (usually $50 \square$ ). The overall impedance at any plane of the system is given the parallel connection of transformed generator impedance $Z$ and transformed cavity impedance. It can be easily shown that the real part of impedance at resonance is reduced by the factor $(1+\square)$.

It follows then

$$
Q_{L}=\frac{Q_{0}}{1+\square}
$$

where $Q_{L}$ is the loaded $Q$ of the cavity/generator assembly. The loaded 3-dB bandwidth $\mathrm{BW}_{\mathrm{L}}$ of the overall combination becomes

$$
B W_{L}=\frac{f_{r}}{Q_{L}}=B W_{0} *(1+\square)
$$

The rf system must provide sufficient generator power to establish the nominal field in the cavity under worst-case conditions, i.e. for full detuning by microphonics. The rf power requirement $\mathrm{P}_{\mathrm{g}}$ can be expressed analytically by [5]

$$
P_{g}=\frac{P_{c}}{4 \square}\left\{(1+\square+b)^{2}+\left[2 Q \frac{\square f}{f}-b \tan (\square B)\right]^{2}\right\}
$$

where $P_{C}$ is the cavity wall dissipation $(42 \mathrm{~W})$, $b$ is the ratio beam power/cavity wall power, $\square_{B}$ is the beam stable phase from crest (0).

For the purpose of investigating capabilities of the system, and adequately specifying components for possible future upgrades, we consider three operating scenarios with varying beam current. Figure 6 shows the necessary generator power for the scenarios of $1 \mathrm{nC}$ at $10 \mathrm{kHz}$, $3 \mathrm{nC}$ at $10 \mathrm{kHz}$, and $3 \mathrm{nC}$ at $100 \mathrm{kHz}$, as a function of the coupling factor, with the detuning $\square f$ as a parameter. 


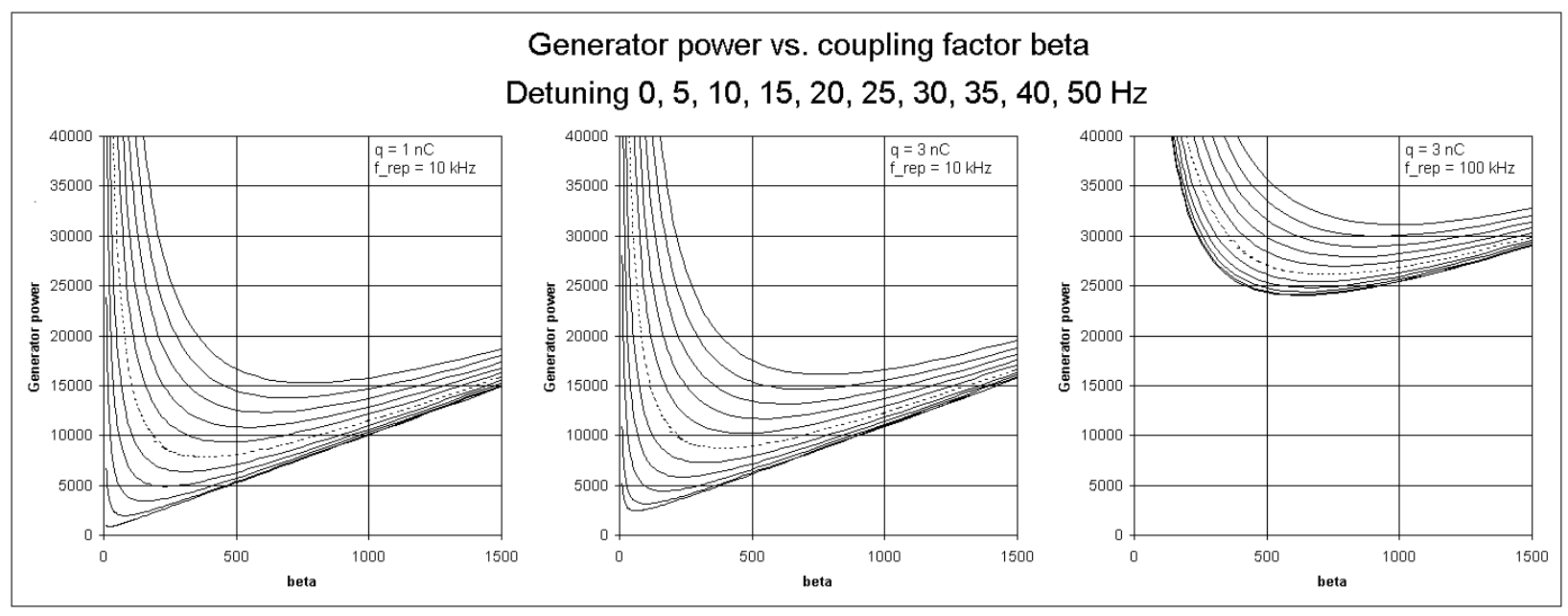

Figure 9-6 Generator power required for $1 \mathrm{nC}$ at $10 \mathrm{kHz}, 3 \mathrm{nC}$ at $10 \mathrm{kHz}$, and $3 \mathrm{nC}$ at 100 $\mathrm{kHz}$, as a function of the coupling factor, and the detuning $\square f$.

For each detuning there exists an optimum coupling factor that leads to minimum generator power. For a given beam current, the generator power increases for increasing tuning deviation. Figure 9-7 shows the generator power for four beam currents under the assumption that the coupling factor has always been adjusted for the respective highest tuning deviation, i.e. following the minima in Figure 6 above. Not surprisingly, the tuning deviation has a particularly important effect at low beam currents.

The key factors for the nominal detuning of $25 \mathrm{~Hz}$ are summarized in Table 9-3 for the three assumed operating scenarios.

Table 9-3 Cavity parameters for nominal detuning $25 \mathrm{~Hz}$

\begin{tabular}{|c|c|c|c|c|c|c|}
\hline $\begin{array}{c}\text { Beam } \\
\text { charge }\end{array}$ & $\begin{array}{c}\text { Beam } \\
\text { Rep. rate }\end{array}$ & $\begin{array}{c}\text { Beam } \\
\text { current }\end{array}$ & $\begin{array}{c}\text { Coupling } \\
\square \text { opt }\end{array}$ & $\begin{array}{c}\text { Loaded Q } \\
\mathrm{Q}\end{array}$ & $\begin{array}{c}\text { Loaded } \\
\mathrm{BW}_{\mathrm{L}}\end{array}$ & $\begin{array}{c}\text { Generator } \\
\text { power }\end{array}$ \\
\hline $1 \mathrm{nC}$ & $10 \mathrm{kHz}$ & $40 \square \mathrm{A}$ & 383 & $26.0 \cdot 10^{6}$ & $50 \mathrm{~Hz}$ & $7.86 \mathrm{~kW}$ \\
\hline $3 \mathrm{nC}$ & $10 \mathrm{kHz}$ & $120 \square \mathrm{A}$ & 398 & $25.1 \cdot 10^{6}$ & $51.9 \mathrm{~Hz}$ & $8.75 \mathrm{~kW}$ \\
\hline $3 \mathrm{nC}$ & $100 \mathrm{kHz}$ & $1200 \square \mathrm{A}$ & 750 & $13.3 \cdot 10^{6}$ & $97.6 \mathrm{~Hz}$ & $26.15 \mathrm{~kW}$ \\
\hline
\end{tabular}




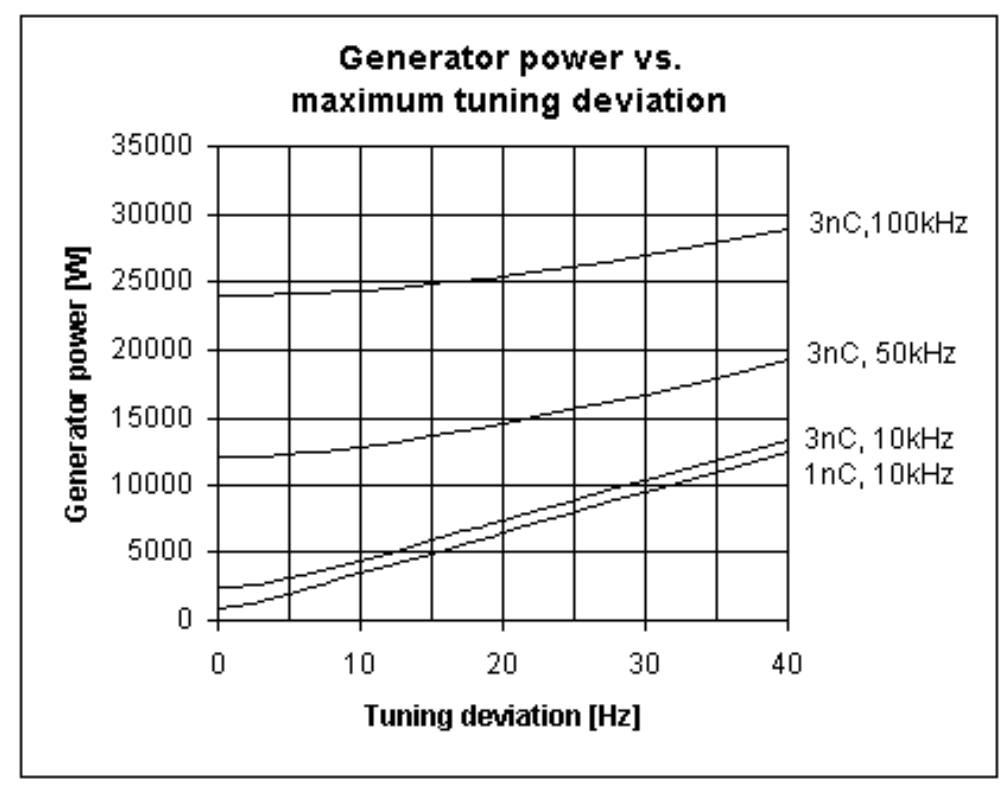

Figure 9-7 Generator power for four beam currents under the assumption that the coupling factor has always been adjusted for the respective highest tuning deviation,

For small beam currents, the optimum loaded bandwidth of the system $Q_{\text {Lopt }}$ coincides with $2^{*} \square$ f. In other words the optimum system bandwidth then just holds the two-sided (double single-sided) frequency deviation. With small beam loading and high $Q$, the magnitude of the cavity conductance is almost equal to the reactive component since the resistive part is negligibly small; the matching condition "magnitude of load conductance = generator conductance $1 / Z$ " then leads to this result.

In all three cases the coupling is significantly weaker than in the standard TESLA cavity, where $\square=3332$ and $Q_{L}=3 \cdot 10^{6}$. This should be no problem since it means withdrawal or shortening of the capacitive power coupler.

Table 9-4 summarizes the baseline main linac requirements, together with the TESLA pulsed system specifications for comparison. 
Table 9-4 Baseline parameters for the recirculating linac, and the TESLA TTF linac specifications

\begin{tabular}{|c|c|c|}
\hline Parameter & $\begin{array}{c}\text { Recirculating } \\
\text { linac }\end{array}$ & TESLA \\
\hline Cavity Length $[\mathrm{m}]$ & 1.038 & 1.038 \\
\hline$E_{a c c}[\mathrm{MV} / \mathrm{m}]$ & 20 & 23.4 \\
\hline Frequency [GHz] & 1.3 & 1.3 \\
\hline$Z T^{2} / Q$ Per Cavity [ $]$ & 1036 & 1036 \\
\hline Flat Top $[\square \mathrm{s}]$ & $\mathrm{CW}$ & 950 \\
\hline Pulse Length $[\square \mathrm{s}]$ & $\mathrm{CW}$ & 1370 \\
\hline Beam Current [mA] & 0.04 & 9.5 \\
\hline Quality Factor $\mathrm{Q}_{0}$ & $10^{10}$ & $10^{10}$ \\
\hline Beam Repetition Rate [Hz] & 10,000 & 5 \\
\hline Duty Factor & 1 & 0.00685 \\
\hline Beam Power Per Cavity $[\mathrm{kW}]$ & 0.83 & 231 \\
\hline Total Beam Power (4 modules) [kW] & 26.6 & 7384 \\
\hline Peak Power Loss Per Cavity [W] & 42 & 57 \\
\hline External Quality Factor $\mathrm{Q}_{\mathrm{ext}}$ & $2.6 \square 10^{7}$ & $2.5 \square 10^{6}$ \\
\hline Bandwidth [Hz] & 50 & 520 \\
\hline Average RF Power Loss Per Cavity [W] & 42 & 0.39 \\
\hline Average RF Load Per Module [W] & 333 & 3.12 \\
\hline Average Power Loss For 4 Modules [kW] & 1.331 & 0.0125 \\
\hline Peak Input RF Power For 4 modules [kW] & 288 & 7,384 \\
\hline
\end{tabular}

\section{REFERENCES}

[1] TESLA Technical Design Report, DESY 2001-011, ECFA 2001-209, TESLAS Report 2001-23, TESLA-FEL 2001-05 (March 2001)

[2] W. Decking, "The TESLA Linear Collider", 2002 Linear Collider Conference, SLAC, California, USA (Feb. 2002)

[3] The NLC Collaboration edited by N. Phinney, "2001 Report on the Next Linear Collider”, LBNL-PUB-47935, SLAC-R-571 (June 2001)

[4] TESLA Report 98-20, pp. 95-97 and private communication

[5] L.Merminga and J.R.Delayen, CEBAF note TH\#96-022 


\section{DEFLECTING CAVITIES}

\section{INTRODUCTION}

RF cavities operating in a dipole mode $\left(\mathrm{TM}_{110}\right.$-like) deflect the head and tail of the $2.5 \mathrm{GeV}$ electron bunches to allow for compression of the x-ray pulse in beamline optics. The center of the electron bunch passes the cavities at zero phase of the rf field such that the head and tail are deflected in opposite directions, and the center of the bunch experiences no deflection. This introduces a divergence into the electrons within a bunch that is much greater than the opening angle of hard x-ray radiation (the radiation opening angle is $7 \mu \mathrm{rad}$ at $1 \AA$ ), and the angular divergence of the electrons $(\sim 6.4 \mu \mathrm{m})$ in the undulators.

The deflecting voltage may be specified in terms of the $\mathrm{x}$-ray pulse duration (see Chapter 1Overview):

where

$$
\begin{gathered}
\square_{x-r a y} \geq \frac{E}{e V_{\square} k_{R F}} \square{ }_{y}^{R F} \sqrt{1+\left(\frac{\square r^{\prime}}{\square \text { undulator }}\right)^{2}} \\
\square_{y}^{R F}=\sqrt{\frac{\Pi}{\square_{R F}}} \quad \square_{r^{\prime}}=\sqrt{\frac{\square_{x-\text { ray }}}{\square L_{\text {undulator }}}} \quad \square_{y}^{\text {undulator }}=\sqrt{\frac{\square}{\square \text { undulator }}}
\end{gathered}
$$

Figure 10-1 shows the dependence of x-ray pulse duration (plotted as full-wdith half maximum) on the deflecting voltage for the baseline parameters (beta-function at the cavities $\square_{\mathrm{RF}}=90 \mathrm{~m}$ beta-function at the insertion devices $\square_{\text {undulator }}=2 \mathrm{~m}$, beam energy $E=2.5 \mathrm{GeV}$, radiation opening angle $\square_{\mathrm{r}}$, beam emittance $\square=9.8 \times 10^{-11} \mathrm{~m}$, and rf wavenumber $\mathrm{k}=82 \mathrm{~m}^{-1}$ ).

There is an advantage in operating the deflecting cavities at high frequency, since for a given peak rf voltage the slope of the voltage waveform around zero-crossing phase is proportional to the frequency of the rf waveform. A practical disadvantage of higher frequencies is the increased resistive losses in the superconducting material (the resistive losses scale as frequency squared).

The voltage required for baseline operation at $2 \mathrm{GeV}$ is approximately $6 \mathrm{MV}$. To obtain an $\mathrm{x}$ ray pulse duration of less than 50 fs for photon energies above $5 \mathrm{keV}$, with a $3.1 \mathrm{GeV}$ electron beam, requires a deflecting voltage of approximately $8 \mathrm{MV}$, and to allow overhead for feedback and reliability, the cavity design voltage is taken to be $8.5 \mathrm{MV}$. Superconducting cavities are a natural choice in obtaining such a large voltage, and the design of a multi-cell structure with large transverse shunt impedance has been developed. A seven-cell $\square$-mode cavity design has been selected. This represents a compromise between a large number of cells to increase transverse shunt impedance and reduce the number of cavities required, and smaller number of cells to minimize the number of cavity modes and potential mode-coupling. The cavity design is similar to a multi-cell deflecting cavity design for the kaon separation project at Fermiliab [1]. 


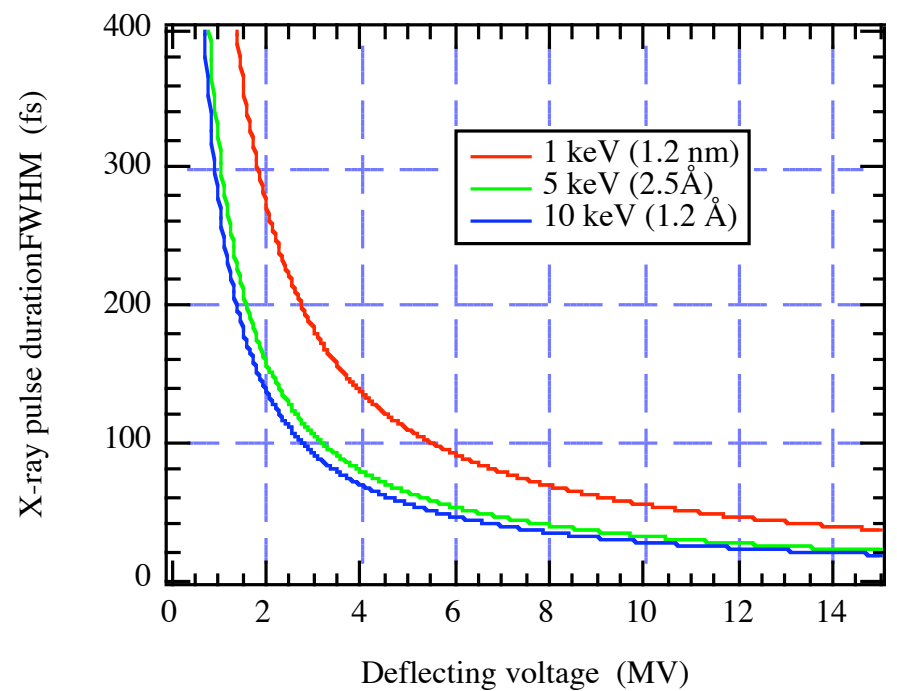

Figure 10-1 X-ray pulse duration as a function of deflecting cavity voltage, for three photon energies, baseline machine parameters.

Figure 10-2 illustrates a typical electromagnetic field distribution of a deflecting dipole mode in a single cell cylindrical pillbox cavity with beampipe, one quarter of the cavity is shown. Preliminary cavity design for the 7-cell cavity has been conducted using the MAFIA and URMEL electromagnetic simulation codes. The cavity operates in a dipole mode with $\square$ phase advance per cell. Due to the presence of the beam iris, the mode is not a pure TM dipole mode, but in a hybrid mode consisting of both TM and TE like modes. For the $\mathrm{TM}_{110}$ mode in an ideal cylindrical pillbox cavity, a beam travelling on-axis experiences a transverse kick from magnetic forces only. Once the beam iris is introduced, as in the present case, both the magnetic and electric fields contribute to the transverse kick, and the forces add in-phase (provided a $\square$ cell-tocell phase advance).

The $\mathrm{TM}_{110}$-like dipole mode is not the lowest frequency mode in the cavity, and there exists a monopole mode (actually a family of modes for a multi-cell cavity) at a lower frequency. These lower order modes (LOM's) could be excited by beam, and their fields may in turn act back on the beam and introduce energy spread. Damping of the LOM's is required, to a level where energy spread is acceptable.
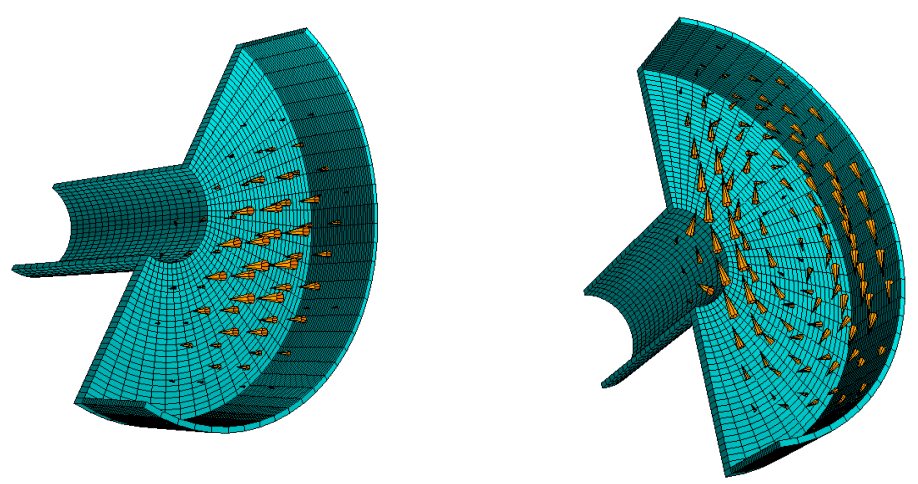

Figure 10-2 Electric (left) and magnetic (right) field distribution of a deflecting dipole mode in a pillbox cavity with beampipes. 
To minimize rf power requirements, reduce short-range wake-field effects, and increase the cavity efficiency, $3.9 \mathrm{GHz} \mathrm{CW}$ superconducting multi-cell dipole rf cavities have been chosen for this application.

\section{Transverse Shunt Impedance}

For the TM dipole mode of an ideal closed cylindrical pillbox cavity, there is no electric field on-axis. Beam going through such a cavity on-axis would experience a transverse force from the magnetic fields only. Once beam irises are introduced, as is necessary for a practical cavity, TElike modes are introduced in the iris (between cells) and beampipe regions. The deflecting mode is no longer a pure $\mathrm{TM}_{110}$, but becomes a hybrid of $\mathrm{TM}_{110}$ and $\mathrm{TE}_{111}$ modes in order to satisfy the new boundary conditions introduced by the irises and beampipes. A beam passing through the cavity on-axis will not just experience transverse forces from the magnetic fields, but also from the transverse electric fields near the iris region. For a cavity with $\square$ phase advance, these two transverse forces add.

To calculate the impedance of the operating mode of the deflecting cavity, we define the transverse shunt impedance as follows,

$$
\begin{gathered}
\left(\frac{R}{Q}\right)_{\square}=\frac{\left|E_{z}\left(r=r_{o}\right) e^{j k_{R F} z} d z\right|^{2}}{\left(k_{R F} r_{o}\right)^{2} \square U}=\frac{V_{\square}^{2}}{\square U} \\
R_{\square}^{*}=\left(\frac{R}{Q}\right)_{\square} Q
\end{gathered}
$$

where the Panofsky-Wenzel theorem has been applied to obtain the deflecting voltage using the off-axis longitudinal electric fields only. $V_{\square}$ is the transverse voltage; $\square=2 \square f$ with $f$ as the resonant frequency; $U$ the stored energy of the mode at the resonant frequency; $E_{z}$ the longitudinal electric field, and $r_{o}$ is the radius at which the longitudinal electric field is integrated along the trajectory $z$. Note that the definition of transverse shunt impedance is in Ohms, and indicates that the deflecting kick is independent of beam position in the cavity.

\section{Preliminary Cavity Design}

Taking advantage of similarities in requirements with the FNAL design for the kaon separation project [1,2], and crab cavity designs developed at KEK and Cornell University [3], the cavity design was initially scaled from the KEK-B crab cavity geometry, and then extended to a 7-cell structure and modeled using the 3-D MAFIA code.

An initial $(R / Q)_{\square}$ of $50 \square /$ cell was obtained for a 7-cell cavity at $3.9 \mathrm{GHz}$ without optimizing the cavity geometry. The $(R / Q)_{\square}$ may be further increased by perhaps $10-15 \%$ with more thorough cavity geometry optimization efforts, which will be performed with the development of the project. 


\section{Simulation Results of a 7-cell Cavity}

The electric field distribution of the deflecting mode is shown in Figure 10-3 as the result of a simulation in 2D ( $r-z$ geometry) using the MAFIA code. The beam pipe radius at the end cell is enlarged to allow for an input power coupler to be added. The end-cell geometry was adjusted (the cavity gap was shortened, and the radius enlarged) in order to obtain good field flatness. This same MAFIA model has also been used for higher- and lower-order mode calculations.

Based on experimental achievements of superconducting cavity technology, a (somewhat conservative) limit of $5 \mathrm{MV} / \mathrm{m}$ gradient in the deflecting field has been chosen, as a result of restricting the critical surface magnetic fields to be around $80 \mathrm{mT}$, (corresponding to a $25 \mathrm{MV} / \mathrm{m}$ acceleration gradient in the TESLA SC cavities). The unloaded quality factor $Q_{0}$ is estimated to be $2 \square 10^{9}$, as measured in tests of a five-cell cavity at Fermilab [2]. Table 1 presents simulation results for one 7 -cell cavity.

To obtain the required 8.5 MV deflecting (peak) voltage, seven 7-cell cavities are required with total rf power dissipation of about 20 watts at $2 \mathrm{~K}$. rf power requirements will depend on the coupler, achievable $Q_{\text {ext }}$ and control of microphonics, and will be assessed in the future.

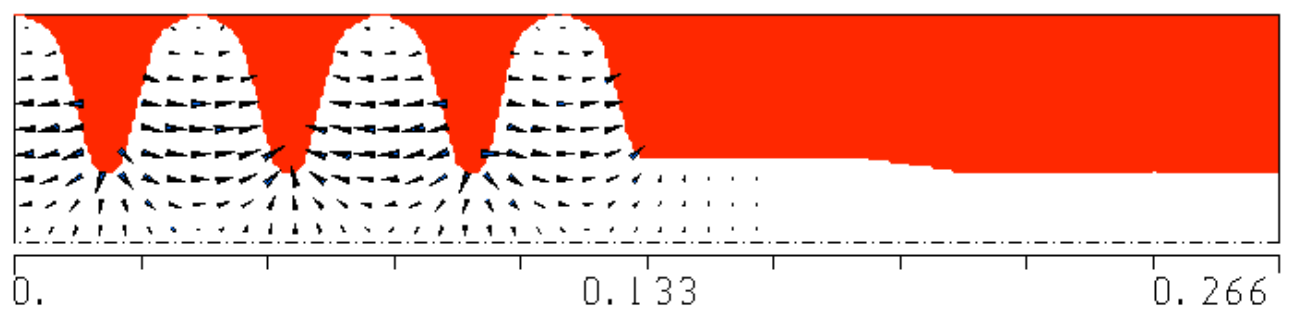

Figure 10-3 Longitudinal electric field distribution of the deflecting mode in the 7-cell cavity at $3.9 \mathrm{GHz}$. The mode was simulated using the 2D MAFIA code using a symmetry plane to model half of the cavity (3.5 cells).

\section{Polarization of the Cavity}

For a perfectly cylindrical rf cavity, there exist a pair of degenerate dipole modes. In order to split the degeneracy sufficiently to avoid coupling between the modes of different polarization, and to orient the required polarization in the vertical direction, the cavity geometry must be perturbed from the purely cylindrical symmetry. We plan to deform the cavity in one plane to achieve these goals, as has been used successfully at KEK and FNAL [2-3] 
Table 10-1 Parameters for one 7-cell SC cavity

\begin{tabular}{|l|c|c|}
\hline Cavity frequency & 3.9 & $\mathrm{GHz}$ \\
\hline Phase Advance per cell & $180^{\circ}$ & Degree \\
\hline Cavity Equator Curvature & 1.027 & $\mathrm{~cm}$ \\
\hline Cavity Radius & 4.795 & $\mathrm{~cm}$ \\
\hline Cell length & 3.846 & $\mathrm{~cm}$ \\
\hline Iris Radius & 1.500 & $\mathrm{~cm}$ \\
\hline Beam pipe radius & 1.500 & $\mathrm{~cm}$ \\
\hline TM mode cut-off frequency & 7.634 & $\mathrm{GHz}$ \\
\hline TE mode cut-off frequency & 5.865 & $\mathrm{GHz}$ \\
\hline$(R / Q)_{\square}$ & 350 & $\Omega$ \\
\hline$Q_{0}$ & $2 \square 10^{9}$ & \\
\hline Transverse shunt impedance & $7 \square 10^{5}$ & $\mathrm{M} \Omega$ \\
\hline Active cavity length & 26.92 & $\mathrm{~cm}$ \\
\hline Effective deflecting gradient & 5 & $\mathrm{MV} / \mathrm{m}$ \\
\hline Transverse voltage & 1.346 & $\mathrm{MV}$ \\
\hline Power dissipation at 2 K & 2.6 & Watts \\
\hline
\end{tabular}

\section{Higher Order Modes (HOMs) and Lower Order Modes (LOMs)}

MAFIA 2D simulations of the 7-cell cavity have been performed to study the higher and lower order modes. Combinations of symmetry and boundary conditions have been applied to simulate all modes below beam pipe cut-off frequencies. Loss factors and shunt impedance of these modes are shown in Tables 10-2 and 10-3. We have assumed $Q_{0}$ of $2 \times 10^{9}$ for all modes, with no damping (a worst case estimation). It is worth noting that both longitudinal and transverse HOMs and LOMs will be damped to some extent by the main rf coupler, and further investigation is underway.

Note that the definition of transverse impedance used in Table 10-2 follows the convention used for beam dynamics and instability calculations, in order to take into account the variation in beam-induced signal as a function of transverse offset from the cavity axis. The induced wakefield dependence on transverse offset is included in the expression:

$$
R_{\square}=\left(\frac{R}{Q}\right)_{\square} Q k_{R F}
$$

where the units are $\Omega / \mathrm{m}$.

A steady state longitudinal voltage, $V_{\text {induce }}$ induced by $1 n C$ bunch at $10 \mathrm{kHz}$ repetition rate is estimated for each monopole mode, and shown in Table 10-3. For the monopole modes, two LOMs dominate, and electric field distributions of these two modes are plotted in Figures 10-4 and10-5. These two dominant lower order monopole modes must be damped to reduce quality factors to below $10^{5}-10^{6}$ in order to maintain an energy spread of $10^{-4}$ at $2.5 \mathrm{GeV}$ beam energy. 
A 3D MAFIA model with rf power coupler will be built to study the damping efficiency of the coupler on all LOM and HOM modes. A coaxial type LOM damper through the beam pipe, proposed for the KEK-B factory, could be adopted if necessary, but this may increase the complexity and cost of the cavity.

\section{Beam Loading of the Deflecting Mode}

Beam passing through deflecting cavity interacts with the cavity, loses energy, and induces a voltage in the cavity. This voltage acts back on the beam and causes energy spread and transverse deflection along the bunch. The beam induced transverse voltage generated in the operating mode may be written as:

$$
V_{\square}=\left(\frac{R}{Q}\right)_{\square} \frac{Q_{o}}{1+\square} k_{R F} \square r I
$$

where $I, \square r$ and $\square$ are beam current, displacement from cavity axis and rf coupling constant, respectively, and baseline parameters are $k_{R F}=82 \mathrm{~m}^{-1}(3.9 \mathrm{GHz}), \square r=0.1 \mathrm{~mm}$ and $\square \square 26$ (assuming an achievable bandwidth of $50 \mathrm{~Hz}$ which corresponds to an external $Q$ of $7.8 \square 10^{7}$ ). For a beam current of $10 \square \mathrm{A}$ in a seven cell cavity, the induced transverse voltage is then $2.1 \mathrm{kV}$, only $0.18 \%$ of the cavity deflecting voltage of $1.2 \mathrm{MV}$.

The power induced in the operating mode of the cavity by beam offset $0.1 \mathrm{~mm}$ is

$$
P=\frac{1}{2} \quad f_{r e p}^{2} q^{2}\left(\frac{R}{Q}\right)_{\square} \frac{Q_{o}}{1+\square} k_{R F} \square r
$$

or $10 \mathrm{~mW}$, and is small compared with 2.6 watts dissipated in the cavity. The loss factor for the operating deflecting mode is $176 \mathrm{~V} /(\mathrm{pC} \mathrm{m})$. 
Table 10-2 Dipole modes (HOM's) of the 7-cell cavity

\begin{tabular}{|c|c|c|c|}
\hline Frequency & $(\mathrm{R} / \mathrm{Q})_{\square}$ & Loss factor & $\begin{array}{c}\text { Transverse } \\
\text { impedance } \mathrm{R}_{\square}\end{array}$ \\
\hline$[\mathrm{GHz}]$ & {$[\square]$} & {$[\mathrm{V} / \mathrm{pC} / \mathrm{m}]$} & {$[\mathrm{M} \square / \mathrm{m}]$} \\
\hline 3.9112 & 0.3 & 0.1 & $4.117 \square 10^{4}$ \\
\hline 3.9212 & 3.0 & 1.5 & $4.862 \square 10^{5}$ \\
\hline 3.9390 & 0.2 & 0.1 & $4.033 \square 10^{4}$ \\
\hline 3.9662 & 0.3 & 0.1 & $4.579 \square 10^{4}$ \\
\hline 4.0045 & 0.3 & 0.2 & $5.756 \square 10^{4}$ \\
\hline 4.0523 & 0.0 & 0.0 & $1.177 \square 10^{2}$ \\
\hline 4.2213 & 0.2 & 0.1 & $3.921 \square 10^{4}$ \\
\hline 4.2939 & 1.2 & 0.7 & $2.093 \square 10^{5}$ \\
\hline 4.4175 & 0.0 & 0.0 & $1.015 \square 10^{3}$ \\
\hline 4.5725 & 1.7 & 1.2 & $3.275 \square 10^{5}$ \\
\hline 4.7488 & 2.7 & 2.0 & $5.316 \square 10^{5}$ \\
\hline 4.9336 & 7.8 & 6.3 & $1.617 \square 10^{6}$ \\
\hline 5.1024 & 1.2 & 1.0 & $2.490 \square 10^{5}$ \\
\hline 5.3002 & 0.3 & 0.2 & $5.823 \square 10^{4}$ \\
\hline 5.3058 & 0.1 & 0.1 & $1.957 \square 10^{4}$ \\
\hline Sum & & 13.7 & $3.720 \square 10^{6}$ \\
\hline
\end{tabular}


Table 10-3 Monopole modes (LOM and HOM) of the 7-cell cavity

\begin{tabular}{|c|c|c|c|c|}
\hline Frequency & $(R / Q)$ & $\begin{array}{c}\text { Loss } \\
\text { factor }\end{array}$ & $\begin{array}{c}\text { Shunt } \\
\text { impedance } \\
\mathrm{R}\end{array}$ & $\begin{array}{c}\text { Induced } \\
\text { voltage }\end{array}$ \\
\hline$[\mathrm{GHz}]$ & {$[\square]$} & {$[\mathrm{V} / \mathrm{pC}]$} & $[\mathrm{M}]]$ & {$[\mathrm{MV}]$} \\
\hline 2.8132 & 1 & 0.0038 & 1,731 & 0.017 \\
\hline 2.8208 & 1 & 0.0056 & 2,507 & 0.025 \\
\hline 2.8321 & 13 & 0.0597 & 26,830 & 0.268 \\
\hline 2.8453 & 10 & 0.0427 & 19,107 & 0.191 \\
\hline 2.8581 & 284 & 1.2742 & 567,621 & 5.676 \\
\hline 2.8685 & 411 & 1.8515 & 821,827 & 8.218 \\
\hline 2.8750 & 56 & 0.2546 & 112,760 & 1.128 \\
\hline 5.7836 & 0 & 0.0017 & 379 & 0.004 \\
\hline 5.8026 & 0 & 0.0002 & 53 & 0.001 \\
\hline 5.8348 & 4 & 0.0357 & 7,783 & 0.078 \\
\hline 5.8797 & 12 & 0.1105 & 23,923 & 0.239 \\
\hline 5.9343 & 5 & 0.0498 & 10,693 & 0.107 \\
\hline 5.9912 & 0 & 0.0002 & 33 & 0.000 \\
\hline 6.0377 & 0 & 0.0013 & 271 & 0.003 \\
\hline 6.6123 & 2 & 0.0233 & 4,496 & 0.045 \\
\hline 6.6135 & 0 & 0.0033 & 633 & 0.006 \\
\hline 6.7391 & 0 & 0.0010 & 185 & 0.002 \\
\hline 6.8025 & 2 & 0.0227 & 4,244 & 0.042 \\
\hline 6.8722 & 0 & 0.0037 & 678 & 0.007 \\
\hline 6.9377 & 0 & 0.0048 & 874 & 0.009 \\
\hline 7.0615 & 32 & 0.3507 & 63,240 & 0.632 \\
\hline 7.5036 & 10 & 0.1124 & 19,077 & 0.191 \\
\hline 7.5093 & 0 & 0.0014 & 243 & 0.002 \\
\hline SUM & & 4.2147 & $1,689,188$ & 16.892 \\
\hline
\end{tabular}

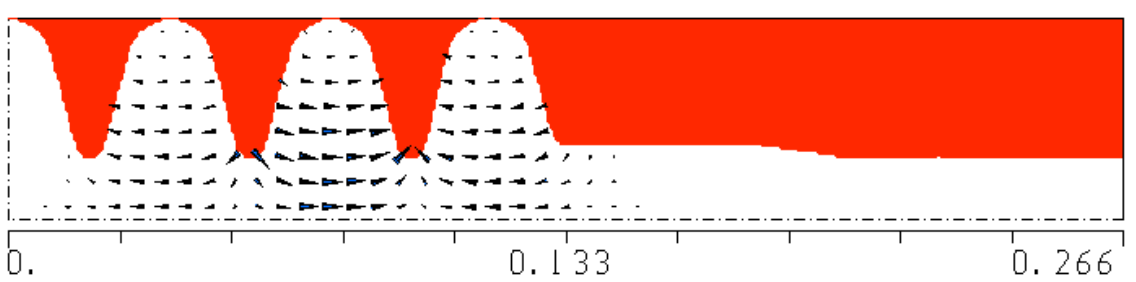

Figure 10-4 Electric field distribution of the monopole LOM at $2.8581 \mathrm{GHz}$ 


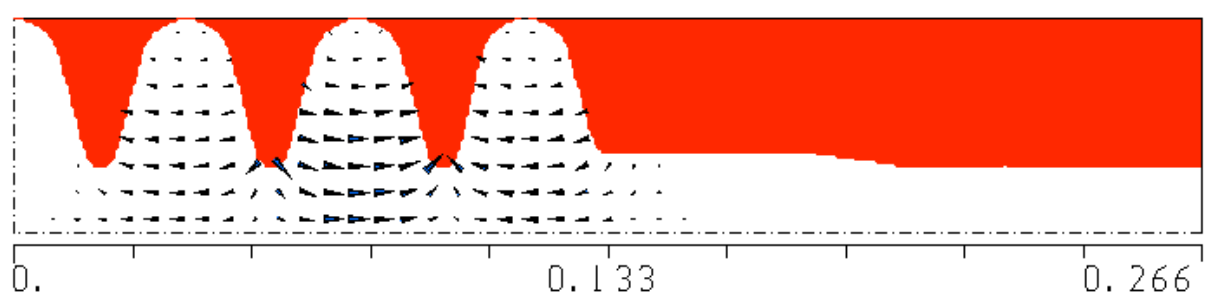

Figure 10-5 Electric field distribution of the monopole LOM at $2.8685 \mathrm{GHz}$

\section{REFERENCES}

[1] L. Bellantoni, et al., "Design and Measurements of a Deflecting Mode Cavity for an RF Separator.” Proc. PAC2001, Chicago, 2001

[2] M. Champion, et al., "Engineering, Design and Prototype Tests of a $3.9 \mathrm{GHz}$ TransverseMode Superconducting Cavity for a Radiofrequency-Separated Kaon Beam," Proc. PAC2001, Chicago, 2001

[3] K. Akai, "Development of Crab Cavity for CESR-B," Proc. PAC 1993, Washington, May 1993. 


\section{LINAC RF SYSTEMS}

\section{Availability of Klystrons}

Presently available L-band klystrons used in other accelerators are listed in Table 11-1. The term "output power" refers usually to the saturated output power but is not consistently defined. This figure should be at least $15-20 \%$ higher than the nominal required power at the cavity input, to take into account losses in the circulator and feeder line, lack of linearity close to the saturation limit, end-of-life condition, etc.

Table 11-1 Available 1.3 GHz CW klystrons

\begin{tabular}{|l|l|l|l|l|}
\hline Manufacturer & Designation & Frequency & $\begin{array}{l}\text { Output } \\
\text { power }\end{array}$ & Where used \\
\hline CPI & VKL7811ST & $1300 \mathrm{MHz}$ & $10 \mathrm{~kW}$ & Stanford, Rossendorf \\
\hline $\begin{array}{l}\text { NORTHROP } \\
\text { GRUMMAN }\end{array}$ & L-4941 & $1497 \mathrm{MHz}$ & $8 \mathrm{~kW}$ & JLab \\
\hline
\end{tabular}

The CPI klystron operates at the desired frequency, it features the highest power output which covers the first project phase adequately. The NORTHROP-GRUMMAN klystron can be converted to the lower frequency, but this involves redevelopment and still results in a unit with smaller output power.

Other manufacturers have been contacted with mostly negative results. THALES delivers a pulsed $1300 \mathrm{MHz}$ klystron of $>40 \mathrm{~kW}$ average power to CERN, but would not consider its conversion to $\mathrm{CW}$ unless a large quantity is involved. TOSHIBA may have suitable items, although their response is pending.

It follows that the VKL7811ST klystron from CPI is presently the best choice pending availability from other manufacturers.

\section{Going to Higher Beam Power}

The possibility of operating at higher beam power is explored in response to possible future needs of the user community. High beam power entails high generator power, and a $10-\mathrm{kW}$ klystron will be at its limit for about $120 \square$ A beam current. CPI will propose upgraded versions of the VKL7811ST unit for 20, 30 and $40 \mathrm{~kW}$ output power. These units are expected to feature electromagnetic instead of permanent-magnetic focusing, to work at higher HV voltage together with lower perveance to improve the efficiency, and may possibly be equipped with an improved output window.

However, increasing the rf power is not necessarily the best approach, since apart from higher investment and operating cost it also involves possible problems with the cavity input coupler, the beam dump, and the cryogenic system, to name but a few.

The rf power requirement may be reduced by several methods: 
Fast tuning of the cavity. This aims at reducing the tuning error by means of e.g. a piezoelectric tuner inside the cavity, or a mechanical/ferrite tuner in the feeder line. This method is most effective at low beam currents but of limited effect at higher intensities (see Figure 9-7 of Chapter 9-Superconducting RF Linacs).

Linac energy management 'LERM'. Cavities with lower than the expected maximum tuning errors require considerably less rf power. At low beam currents, a perfectly tuned cavity needs only half the amplifier power compared to a unit with the maximum admissible tuning error. This follows from the fact that the tuning error limit for optimum coupling coincides with the 3dB bandwidth point, and can also be seen in Figure 9-6 of Chapter 9-Superconducting rf Linacs. The strategy for a population of cavities with different microphonics errors is to attribute a larger fraction of the total required voltage to the well-tuned cavities while decreasing the fraction to off-tune units. An automated optimization algorithm is reported to have achieved a benefit of about $25 \%$ average amplifier power [1].

RF energy recovery. The high-energy beam is not dumped but decelerated by being returned through the linac with phase reversal to deliver its energy back to the system. Due to their long time constant, the SC cavities integrate over the accelerating and decelerating cycle such that the effective beam current is reduced by an order of magnitude or more. Besides the advantages of reducing the power in the coupler and in the cryogenic system, the dumping of the beam at low energy $(10 \mathrm{MeV})$ is also very attractive from a radiation protection point of view. The drawback, however, is the necessity of a beam return line to recirculate the beam after the final arc, and the additional complexity of controlling the beam through four more passes.

A possible development scenario is therefore to start the project with low beam charge and low repetition rate ( $1 \mathrm{nC}$ and $10 \mathrm{kHz}$ respectively) which can be safely handled by the available $10-\mathrm{kW}$ klystrons. Operation at higher beam intensity is possible by voltage distribution shaping, the introduction of fast tuners, and eventually by rf power recovery. This should allow the beam current to be increased gradually to an ultimate design goal of $1200 \square \mathrm{A}$, without the need of higher-power klystrons. This does not mean however that the possibility of using improved klystrons from the beginning should be discarded. 


\section{RF System Layout}

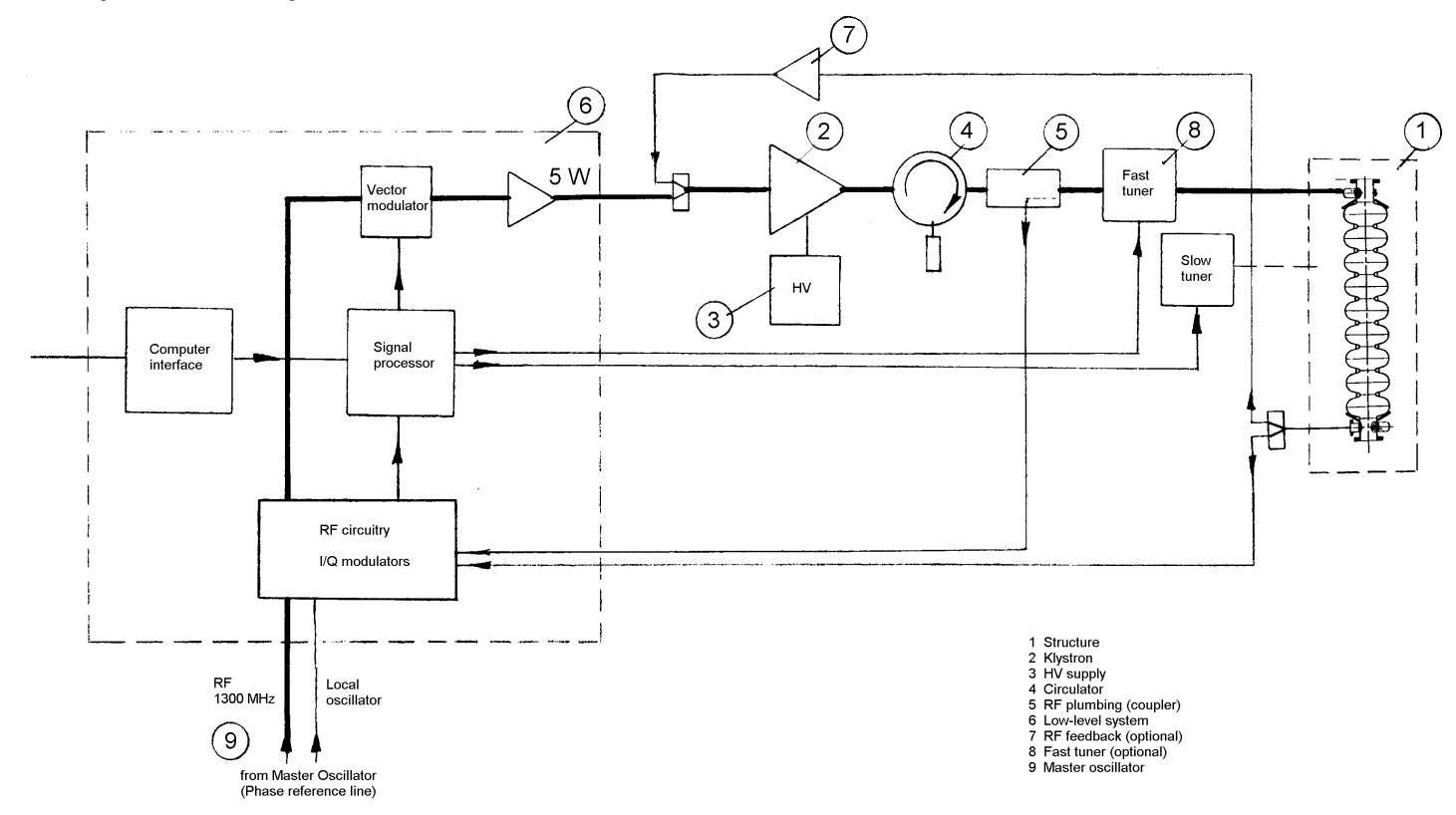

Figure 11-1 Linac rf system schematic layout

The design of the rf system follows well established principles, and the schematic layout is shown in Figure 11-1. The only element of predetermined limited lifetime is the klystron, for which no semiconductor equivalent (yet) exists. All elements are of course subject to "unscheduled maintenance" but are well within standard technology.

Comments to the different parts of the system:

1. Accelerating structure. A cryostat contains eight or more 9-cell TESLA cavities, together with accessory equipment such as steering magnets. As the design of cavities and cryostat is constantly improved in the context of other large projects, the final configuration will depend on the state of the art at project time.

2. Klystron. (see above).

3. HV supply. There is a choice of two technologies, namely switching supplies (cheap, efficient, low stored energy and flexible, but prone to high ripple) vs. continuously regulated supplies (opposite characteristics). The steady progress in semiconductor and ferrite technology had a major impact on the structure of switching power supplies which become lighter and smaller due to ever-increasing switch frequencies. The voltage of the presently available klystrons $(16 \mathrm{kV})$ is relatively low which alleviates insulation and maintenance problems.

4. Circulator. This ferrite-loaded non-reciprocal device protects the klystron by diverting the reflected wave from the highly mismatched cavity input port to a separate power absorber.

5. RF plumbing. The entire high-power rf distribution is composed of WR650 waveguide components, except where building constraints may dictate short sections of coaxial lines. 
The size of the waveguides is determined by the operating frequency. Due to the large dimensions their breakdown limit is much higher than the operational fields, therefore no special precautions such as pressurization are necessary.

6. Low-level system. Consisting of two parts:

Analog rf circuitry. Standard analog building blocks such as power splitters, mixers and filters are used to manipulate and distribute the operational frequency, based on the signals received from the master oscillator. A vector modulator for the control of amplitude and phase, and a 5-W semiconductor amplifier are the most prominent parts in the main signal path that leads to the input of the klystron. Other analog modules perform the signal conditioning to transform the signals received at $1300 \mathrm{MHz}$ to a frequency around $80 \mathrm{MHz}$ for treatment by the digital controller.

Digital signal processor. This is the part where the progress in signal handling and information processing leads to spectacular results in terms of system diagnostic possibilities and operational flexibility. The conditioned signals at the frequency of $\sim 80 \mathrm{MHz}$ are converted into digital form by A/D converters, treated by the digital signal processor, passed through D/A converters and finally routed to the outputs. These include in particular the vector modulator for control of the cavity field, together with actuators such as the cavity tuner.

An accelerator rf system comprises at least three interacting feedback loops, namely for cavity amplitude, cavity phase and resonator tuning. All of these feedback loops are implemented here in a fully digital form to allow the easy introduction of different control algorithms by software. While the control of each cavity is self-contained and local, a fast interface allows remote operation and data collection by a central computer. A separate section of the digital controller takes on the mundane tasks of an interlock system such as switching the system ON/OFF and to protect the equipment in case of failure.

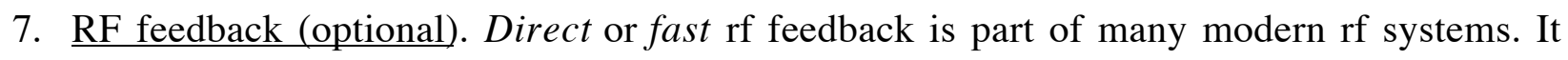
permits an increase in the small-signal rf bandwidth and linearizes the transfer function of the klystron. Its implementation for a 9-cell cavity is more complicated than for a single cell due to the adjacent secondary resonances but proven solutions exist to solve this problem [2]. It is an optional device that can be added at a later stage.

8. Fast tuner (optional). The aim of a fast tuner is to reduce the rf power requirements by decreasing the cavity tuning deviations. It can be implemented as a mechanical or as an electronically controlled ferrite-based device, and is most effective at low beam currents as explained above.

9. Master oscillator and phase reference line. All linac rf chains receive an input signal from a phase reference line to assure phase coherence in the different resonators. The reference signal is created in a common master oscillator and distributed by a temperaturecompensated line that runs along the linac structures. Directional couplers in the pickup points together with a low-reflection generator assure a high degree of mutual decoupling. It is planned to derive the master oscillator from the optical laser resonator to meet the stringent synchronization requirements. 


\section{Power Consumption}

The necessary peak $\mathrm{AC}$ power to generate the maximum rf power of $10 \mathrm{~kW}$ is determined by the efficiencies of the klystron $(\sim 33 \%)$ and the HV power supply $(\sim 90 \%)$; it amounts to about $33.7 \mathrm{~kW}$. It is expected that the average power consumption for low beam current is about $25 \%$ lower. Power requirements for ancillary devices such as filaments, blowers, ion pump on the klystron etc. are estimated at 500 watts leading to a total of about $34.2 \mathrm{~kW}$ peak, $27.5 \mathrm{~kW}$ average. The total requirement for the $32 \mathrm{rf}$ chains of the main linac is therefore $1.1 \mathrm{MW}$ peak, $0.88 \mathrm{MW}$ average.

\section{Conclusions}

We find that a net klystron power of $8 \mathrm{~kW}$ per cavity is required to accelerate $1 \mathrm{nC}$ bunches in four passes at a repetition rate of $10 \mathrm{kHz}$. Substantially larger beam currents can still be handled with the same klystrons but will ultimately require rf power recovery by a return line. The main linac rf parameters are summarized in Table 11-2.

\section{Table 11-2 Main linac parameters}

\begin{tabular}{|l|l|}
\hline Energy gain per pass in main linac & $600 \mathrm{MeV}$ \\
\hline Accelerating gradient in main linac & $20 \mathrm{MVm}{ }^{-1}$ \\
\hline Design voltage per cavity & $20 \mathrm{MV}$ \\
\hline Duty factor & $\mathrm{CW}$ \\
\hline Wall power losses per cavity & $42 \mathrm{~W}$ \\
\hline rf losses per cryomodule & $336 \mathrm{~W}$ \\
\hline Average beam current $(4$ passes $)$ & $40 \square \mathrm{A}$ \\
\hline Beam power per cavity & $800 \mathrm{~W}$ \\
\hline Cavity $\mathrm{Q}_{0}$ (unloaded) & $1 * 10^{10}$ \\
\hline Cavity $\mathrm{R} / \mathrm{Q}$ (“linac" definition, $\left.\mathrm{P}=\mathrm{V}^{2} / \mathrm{R}\right)$ & $1036 \square$ \\
\hline Cavity coupling factor $\square$ & 384 \\
\hline Cavity $\mathrm{Q}_{\mathrm{L}}$ (loaded) & $26 \mathrm{x} 10^{6}$ \\
\hline Cavity loaded bandwidth & $50 \mathrm{~Hz}$ \\
\hline Total main linac rf power & $320 \mathrm{~kW}$ peak, $256 \mathrm{~kW}$ average \\
\hline Total main linac AC power $\left(\square_{\text {overall }}=29.7 \%\right)$ & $1.1 \mathrm{MW}$ peak, $0.9 \mathrm{MW}$ average \\
\hline
\end{tabular}

\section{REFERENCES}

[1] J. R. Delayen, L. R. Doolittle, C. E. Reece, “Operational optimization of large-scale srf accelerators," Proc. 1999 Particle Accelerator Conference, New York, 1999

[2] D. Boussard, H.P. Kindermann, V. Rossi, "RF Feedback applied to a Multicell Superconducting Cavity," Proc. EPAC 88, June 1988, Rome, Italy. 


\section{PHOTOCATHODE GUN RF SYSTEMS}

The rf power requirements of the 4-1/2 cell normal conducting electron injector are summarized in Table 12-1. The figures refer to a $5 \mu \mathrm{s}$ rf pulse, i.e. the filling time is included in the power required to achieve gradient (see Chapter 5-RF Photocathode Gun)

Table 12-1 RF power requirements of the electron injector:

\begin{tabular}{|l|c|c|}
\hline \multirow{2}{*}{\multicolumn{1}{c|}{ Resonator }} & \multicolumn{2}{c|}{ rf power requirement } \\
\cline { 2 - 3 } & Peak & Mean \\
\hline Rf-gun & $1.2 \mathrm{MW}$ & $60 \mathrm{~kW}$ \\
\hline Accelerating cavity 1 & $2.5 \mathrm{MW}$ & $125 \mathrm{~kW}$ \\
\hline Accelerating cavity 2 & $2.5 \mathrm{MW}$ & $125 \mathrm{~kW}$ \\
\hline Accelerating cavity 3 & $2.5 \mathrm{MW}$ & $125 \mathrm{~kW}$ \\
\hline Total & $\mathbf{8 . 7} \mathbf{M W}$ & $\mathbf{4 3 5} \mathbf{~ k W}$ \\
\hline
\end{tabular}

To obtain maximum flexibility for the adjustment of the flat beam parameters, the preferred solution is an arrangement of four independent rf chains. This allows full advantage to be taken of fast individual control of each resonator by a digital controller acting directly on a low-power vector modulator, avoiding the use of a high-power rf distribution network with ferrite phase shifters and attenuators.

The structure of each chain is then very similar to the linac rf systems shown in Chapter 5-RF Photocathode Gun with a low power regulating system implemented in digital technology feeding the high-power part consiting of klystron, HV supply or modulator and circulator. The high-power part, however, is operating in pulsed rather than $\mathrm{CW}$ mode which leads to specific design considerations for the HV power supply (see Modulator below).

\section{KLYSTRON REQUIREMENTS}

The requirements for the klystrons remain well within reasonable technological limits. Units have been built at much higher and lower frequency bands with substantially higher output powers, in terms of peak as well as average ratings. The choice in L-band is nevertheless rather limited. A klystron that comes close to our requirements is the E3718 from TOSHIBA, described in Table 12-2 [1]. It has been built in small quantities for the Japan Nuclear Cycle Development Institute (JNC), and could be used for our proposed facility after re-tuning from $1249 \mathrm{MHz}$ to the frequency of $1300 \mathrm{MHz}$. The maximum power output covers the requirement with large reserve, even taking unto account that the useable range is only about $90 \%$ of the indicated saturated power limit. The system design considerations are tentatively based on this klystron, pending availability of suitable devices from other manufacturers. This choice may be revised later. 
Table 12-2 TOSHIBA klystron E3718

\begin{tabular}{|c|c|c|}
\hline Operation mode & CW & Pulsed \\
\hline Rf frequency & \multicolumn{2}{|c|}{$1.249 \mathrm{GHz}$} \\
\hline Rf power & $1.2 \mathrm{MW}$ & $4.2 \mathrm{MW}$ \\
\hline Efficiency & $65 \%$ & $50 \%$ \\
\hline Beam voltage & $90 \mathrm{kV}$ & $147 \mathrm{kV}$ \\
\hline Cathode current & $25 \mathrm{~A}$ & $56.5 \mathrm{~A}$ \\
\hline Drive power & $12 \mathrm{~W}$ & $15 \mathrm{~W}$ \\
\hline Pulse width & - & $800 \square \mathrm{s}$ \\
\hline Repetition rate & - & $250 \mathrm{~Hz}$ \\
\hline
\end{tabular}

Even though the required power for the rf-gun is less than half of those for the cavities, it is proposed to provide the same rf system in all four chains. This allows overpowering of the resonator of the electron gun to reduce the filling time. It also enhances maintainability and simplifies the logistics.

\section{MODULATOR REQUIREMENTS}

Taking the klystron described above as a realistic starting point, the required modulator voltage is $150 \mathrm{kV}$, together with a peak output power of $5 \mathrm{MW}$ for a klystron efficiency of $\square=0.5$, augmented by $10 \%$ reserve. The requirements for a single rf system are given in the Table $12-3$.

Table 12-3 Modulator requirements

\begin{tabular}{|l|l|}
\hline Parameter & Required values \\
\hline \hline Modulator voltage & $150 \mathrm{kV}$ \\
\hline Modulator peak power & $5.5 \mathrm{MW}$ \\
\hline Pulse repetition time & $100 \square \mathrm{s}($ rep. rate $10 \mathrm{kHz})$ \\
\hline Pulse duration & $5-7 \square \mathrm{s}$ \\
\hline Pulse rise time & $500 \mathrm{~ns}$ \\
\hline Duty factor & $5-7 \%$ \\
\hline Modulator average power & $275-385 \mathrm{~kW}$ \\
\hline
\end{tabular}


An adequate design goal for the risetime of the modulator pulse is $\sim 500 \mathrm{~ns}$. This allows consideration of the full range of modulators, based on thyratrons or on semiconductors, where the rate of current rise is a limiting factor. On the other hand, this risetime is still short in comparison with the rf pulse length, and it leads to a reduction of less than $3 \%$ in the cavity voltage at the end of the $5 \square \mathrm{s}$ pulse for a cavity with $Q_{0}=24000$ (less reduction for lower $Q_{0}$ ). Alternatively the klystron beam can be switched on $500 \mathrm{~ns}$ earlier than the rf pulse to get full $\mathrm{rf}$ power capabilities immediately and maximum cavity field, at the expense of a $10 \%$ increase in the average power consumption. Either possibility or combination thereof is acceptable, with the best compromise to be found in practical operation.

If the stored energy in the gun cavities is to be removed actively by reversing the drive phase after the bunch has been accelerated, the nominal $5 \mu \mathrm{s}$ pulse is extended to $7 \mu \mathrm{s}$, as shown in Table 12-3.

\section{Implementation of the modulator}

The key element in the modulator is the high-power switch. One elegant possibility to pulse a hypothetical custom-built klystron is to provide internal beam modulation capabilities, such as a modulating anode or a laser-driven photocathode. However for existing klystrons external pulsing is necessary. Excluding more exotic possibilities like a hard-tube modulator or a Hobertron (Hollow beam valve) there remains a choice between vacuum-tube technology and semiconductor technology. Three feasible designs are presented, namely two line type modulators based respectively on a thyratron and on a semiconductor thyratron replacement switch, and finally an induction modulator with composite semiconductor switch.

A. Line type modulator with thyratron switch. This well-proven technology consists of the following modules [2]:

A1.Thyratron: This is a Turn-ON only switch, based on triggered avalanche conduction in a hydrogen or deuterium-filled tube. Conduction has to be stopped by current reversal in the external circuit which determines the pulse duration. This is performed by a pulse forming network (PFN) that is described below.

For the present application there are two main critical thyratron parameters:

- The time necessary for the de-ionization of the discharge path. This limits the applicable pulse rate in standard units to $400 \mathrm{~Hz}$, respectively to $50 \mathrm{kHz}$ in special units.

- The average current, which determines the average amount of ion bombardment of the cathode. It is usually limited to a few amperes, whereas the requested rating is of the order of $10 \mathrm{~A}$.

Either limitation may be overcome by means such as extensive de-rating or parallel connection of several klystron/PFN assemblies into a common pulse transformer.

Table 12-4 gives the thyratron specifications required, supposing a PFN charged to $40 \mathrm{kV}$. Parameters of readily available units are shown in columns 3 and 4. 
Table 12-4 Thyratron parameters

\begin{tabular}{|c|c|c|c|}
\hline \multirow{2}{*}{ Parameter } & \multicolumn{3}{|c|}{ Value } \\
\cline { 2 - 4 } & $\begin{array}{c}\text { Proposed } \\
\text { system }\end{array}$ & $\begin{array}{c}\text { CX2412 } \\
\text { (Marconi) }\end{array}$ & $\begin{array}{c}\text { CX 1635A } \\
\text { (Marconi) }\end{array}$ \\
\hline \hline Peak voltage $[\mathrm{kV}]$ & 40 & 60 & 20 \\
\hline Peak current [A] & 275 & 15000 & 1000 \\
\hline Average current [A] & 13.8 & 15 & 1 \\
\hline Rate of current rise $[\mathrm{kA} /[\mathrm{s}]$ & 0.7 & 75 & 100 \\
\hline Pulse rate $[\mathrm{kHz}]$ & 10 & 5 & 50 \\
\hline
\end{tabular}

It can be seen that all specifications except the pulse rate can be met by the thyratron CX 2412, and that on the other hand a fast thyratron such as the CX 1635A falls short in terms of power handling capability (peak voltage and average current). The ratio (average current)/(peak current) of thyratrons is generally of the order of $10^{3}$, whereas the application requires $\geq 5 \times 10^{3}$. The choice of a thyratron in this case is therefore governed by the available average power, with a large reserve in peak power.

It is possible that a thyratron can be found that meets all the specifications in a single unit, but the use of two thyratrons in parallel has to be envisaged. Investigations with a manufacturer are under way.

A2. Pulse forming network (PFN): The PFN is essentially a low-pass LC network composed of HV capacitors and inductors, optimized to deliver a near to ideal square pulse of a given duration when discharged. The PFN behaves like a transmission line with a characteristic impedance $Z$ and a delay time $\square$ which is half the required pulse duration due to the full pulse reflection at the far end. Here $Z=20 \times 10^{3} / 275=72.7 \square$ and $\square=2.5 \square \mathrm{s}$.

The load has to be matched to the characteristic impedance $Z$, the output voltage is then half the charging voltage. The $V^{*} A$ rating of the switch has therefore to be twice the load power.

The capacitors should exhibit low series inductance and use self-healing dielectric. The coils are of the air-core type, mutually coupled and individually adjustable by field displacement plungers.

The ratio between voltage and current of the switch determines the characteristic impedance of the line. It can be varied over a wide range to match all requirements. The number of LC sections determines the quality of the output pulse in terms of risetime and flatness of the pulse top. Variable characteristic impedance along the line may be necessary to compensate for the droop caused by the magnetizing current of the pulse transformer. The PFN may pose some technical problems but is not a critical element.

A3. Pulse transformer A pulse transformer is necessary to bridge the gap between the very high voltage needed by the klystron and the lower voltage that practical switches can sustain. Pulse transformers with step-up ratios 1:3 to 1:25 are in current use. Since klystrons are operated 
with the anode at ground potential, the cathode together with the filament connection is at high negative potential with respect to ground. A bifilar or coaxial secondary winding is therefore necessary to carry the filament power to the cathode. The pulse transformer is usually housed in an oil-filled cubicle for reasons of $\mathrm{HV}$ insulation, cooling and reduction of acoustic and electromagnetic interference.

For a PFN charging voltage of $40 \mathrm{kV}$ the resulting primary voltage is $20 \mathrm{kV}$ and a transformer ratio of 1:7.5 is necessary to achieve a secondary voltage of $150 \mathrm{kV}$. A practical design has been devised and described in Reference [2]. It is assembled from three cores, each of outer diameter $=500 \mathrm{~mm}$, inner diameter $=200 \mathrm{~mm}$, height $=100 \mathrm{~mm}$, wound of METGLAS 2605SA1, 20 micron thick, from Allied-Honeywell. The high permeability of this material leads to a low magnetizing current, and the high saturation limit of $2.5 \mathrm{~T}$ allows a low number of turns to be used. This feature is important to minimize the stray inductance, which is further minimized by the winding layout. The primary winding consists of two legs of 3 turns each, connected in parallel and distributed over the circumference; the secondary of 30 turns is arranged with variable spacing according to the increasing voltage from turn to turn.

The calculated stray inductance of this winding layout is only $\sim 45 \quad \square \mathrm{H}$, supposing that the entire core cross-section is free of magnetic flux when the primary winding is short-circuited. The real conditions depend critically on the layout of the primary winding and are therefore difficult to take into account. Even if the real stray inductance is twice as large the resulting transformer risetime of $\sim 67 \mathrm{~ns}$ is still small compared to the permissible overall risetime of $500 \mathrm{~ns}$. This large margin can either be used to reduce the rate of current rise in the switching element by slowing down the input pulse, or the number of transformer turns can be increased to reduce the core losses and the magnetizing current. The best compromise has to be found by practical tests.

The cores are operated at 0.025 volt-seconds, which together with an effective core crosssection of $31 * 10^{-3} \mathrm{~m}^{2}$ leads to a magnetic induction swing of $0.806 \mathrm{~T}$. The core loss at this modest induction is estimated at about $100 \mathrm{~J} / \mathrm{m}^{3}$ for the pulse duration of $5 \square \mathrm{s}$, the total dissipation in the effective core volume of $34.7 \times 10^{-3} \mathrm{~m}^{3}$ is then about $3.5 \mathrm{~J}$ per pulse or about $4 \mathrm{~J}$ per pulse together with the copper losses. At a pulse repetition rate of $10 \mathrm{kHz}$, the transformer losses amount to $40 \mathrm{~kW}$.

If better performing but more expensive core material such as $2714,2705 \mathrm{M}$ or Finemet is chosen, then the core losses could be reduced to less than 2 Joules per pulse for an efficiency of about $95 \%$.

Could a single pulse transformer feed all four klystrons in parallel? The core losses remain the same, but the copper losses would increase by an order of magnitude, and the risetime by a factor of four. A detailed technical study is needed to ponder this possibility. The same applies for an increase of the pulse length to $6 \square \mathrm{s}$ to allow one additional microsecond for phase reversal at the end of the rf pulse.

Going to a significantly higher repetition rate, however, would necessitate a complete rethinking of the pulse transformer to raise the efficiency and master the cooling problems. 
A4. Resonant charger: essentially a switched inductor that transmits charge from a storage capacitor to the pulse forming network (PFN), doubling the voltage in the process. The value of the inductor is chosen such that the resulting resonant charging current resets the core of the pulse transformer.

A5. Charging supply: A regulated AC/DC converter, which rectifies the $60 \mathrm{~Hz}$ supply voltage and charges a storage capacitor to $20 \mathrm{kV}$. The precision of this DC voltage determines the precision of the pulsed output voltage of the modulator. The charging supply with average $\mathrm{AC}$ input power of about $350 \mathrm{kVA}$ is at the same time the major cost element.

B. Line-type modulator with solid-state "thyratron replacement" switch.

The tendency to replace thyratrons in existing systems by solid-state devices is in full swing. Switch modules containing 3 to 6 thyristors or SCR's together with drive electronics and rated 10 to $12 \mathrm{kV}$ are available from at least two manufacturers $[3,4]$. The switches are of the Turn-ON only type therefore a PFN is needed as before, the topology of the modulator remains unchanged.

Table 12-5 shows the relevant parameters for the two available semiconductor modules in columns 3 and 4, column 2 giving again the project requirements. In both cases the series connection of three modules is assumed to comply with a PFN charging voltage of $27 \mathrm{kV}$. With these devices the rms current has to be taken as a yardstick of multipulse performance (contrary to thyratrons where the average current is the limit).

Table 12-5 Parameters of available "Thyratron replacement" solid-state switches

\begin{tabular}{|l|l|l|l|}
\hline \multirow{2}{*}{ Parameter } & \multicolumn{3}{c|}{ Value } \\
\cline { 2 - 4 } & $\begin{array}{l}\text { Propose } \\
\text { d system }\end{array}$ & $\begin{array}{l}\text { "10 kV module" } \\
\text { (Applied Pulse } \\
\text { Power) }\end{array}$ & $\begin{array}{l}\text { EFM-SPR } \\
\text { 08F4500-6-WC } \\
\text { (Asea Brown } \\
\text { Boveri) }\end{array}$ \\
\hline \hline Peak voltage [kV] & 27 & $3 \times 10$ & $3 \times 12$ \\
\hline Peak current [A] & 407 & 8000 & 1500 \\
\hline RMS current [A] & 91.1 & $>35$ (not tested) & 94.8 \\
\hline Rate of current rise $[\mathrm{kA} / \square \mathrm{s}]$ & 1.02 & 30 & 1.5 \\
\hline Pulse rate $[\mathrm{kHz}]$ & 10 & $>0.06$ (not tested) & $>0.4$ (not tested) \\
\hline
\end{tabular}

The $10 \mathrm{kV}$ module from Applied Pulse Power has an impressive margin in peak power capability. Maximum rms current and maximum pulse rate are neither tested nor specified. According to the manufacturer, the given data represent the limits of the test power supply rather than the device. Successful tests with the series connection of three modules are reported, in normal mode and under fault conditions. 
The module EFM-SPR 08F4500-6-WC from Asea Brown Boveri meets all requirements except pulse rate. Again it is not clear whether the latter represents a limit of the device or the test facilities. The possibility of series connection is explicitly mentioned in the device description.

Both devices show promise for the present application. In general, the ratio of mean power/ peak power is considerably larger than in thyratrons, hence these devices are more suitable for high duty factor. It is conceivable that the apparent pulse-rate limit is the result of the test setup restraints but does not reflect the inherent capabilities of these devices. According to catalog data the circuit-commuted turn-off time of similar devices is of the order of $\sim 50 \square$ s therefore operation at $10 \mathrm{kHz}$ and higher should be possible, but the maximum obtainable pulse rate remains a critical parameter.

\section{Induction modulator with semiconductor ON-OFF switch}

This approach is based on Turn-OFF switches such as IGBTs (Isolated Gate Bipolar Transistor) or MOSFETS (Metal Oxide Semiconductor Field Effect Transistor). They allow full control of current flow, into the conducting as well as non-conducting state. The pulse length is no longer determined by a pulse forming network, but by the drive pulse to the switches which can be adjusted by computer control. These devices work at peak voltages up to $3 \mathrm{kV}$.

The operating principle of the induction modulator or inductive adder is similar to an induction linac. It permits a high voltage transformation ratio to be obtained with low stray inductance, resulting in short pulse risetime. A secondary winding passes through a number of induction cores which are driven individually, enclosing their total rate of change of magnetic flux, hence adding the output voltages. An arrangement of $n$ individual cores is also said to consist of $n$ (or $1 / n)$ fractional turns with respect to the common secondary. While the initial concept is based on a single turn in primary and secondary windings, the method is also applicable to an arbitrary number of either turns, to accommodate electromagnetic and dimensional constraints.

Table 12-6 gives in columns 3 and 4 the characteristics of induction modulators of existing and proposed systems. Column 3 shows the parameters of the $C L W$ modulator (named after its inventors, Crewson, Lindholm and Woodburn) that is used in a commercial cancer treatment system from Scanditronix and in the Japan National Cancer Center [5]. The power is controlled by eight $1-\mathrm{kV}$ modules equipped with IGBT switches, which take the required energy from a common storage capacitor. A sophisticated winding scheme for the primary turns is used to assure that at least two independent switches feed each fractional core, to assure that the core is always energized and to prevent overvoltages in case of trigger malfunction.

Column 4 shows the data of a replacement project for the conventional SLAC 5045 klystron modulator [6]. The huge peak output power is needed as a common source for eight klystrons. Each core is driven by two PC boards with one $3.3 \mathrm{kV}$ IGBT per board that can be easily exchanged for maintenance. Freewheeling reverse diodes protect the semiconductor switches. 
Table 12-6 Parameters of induction modulators

\begin{tabular}{|l|l|l|l|}
\hline \multirow{2}{*}{ Parameter } & \multicolumn{3}{c|}{ Values } \\
\cline { 2 - 4 } & $\begin{array}{l}\text { Proposed } \\
\text { system }\end{array}$ & $\begin{array}{l}\text { CLW } \\
\text { modulator } \\
\text { (operating) }\end{array}$ & $\begin{array}{l}\text { SLAC 5045 } \\
\text { Replacement } \\
\text { (Project) }\end{array}$ \\
\hline \hline Peak output voltage & $-150 \mathrm{kV}$ & $-140 \mathrm{kV}$ & $-500 \mathrm{kV}$ \\
\hline Peak output current & $36.7 \mathrm{~A}$ & $95 \mathrm{~A}$ & $2120 \mathrm{~A}$ \\
\hline Peak output power & $5.5 \mathrm{MW}$ & $13.3 \mathrm{MW}$ & $1060 \mathrm{MW}$ \\
\hline Duty factor & $50 * 10^{-3}$ & $3 * 10^{-3}$ & $0.36 * 10^{-3}$ \\
\hline Average power & $275 \mathrm{~kW}$ & $40 \mathrm{~kW}$ & $382 \mathrm{~kW}$ \\
\hline Pulse width & $5 \square \mathrm{s}$ & $3 . .10 \square \mathrm{s}$, & $3 \square \mathrm{s}$ \\
& variable & \\
\hline Pulse risetime & $0.5 \square \mathrm{s}$ & $0.4 \square \mathrm{s}$ & $0.4 \square \mathrm{s}$ \\
\hline Pulse repetition rate & $10 \mathrm{kHz}$ & $0.3 \mathrm{kHz}$ & $0.12 \mathrm{kHz}$ \\
\hline Number of secondary turns & 30 & 70 & 3 \\
\hline Number of "fractional" turns & 3 & 2 & 80 \\
\hline Switches / fractional turn & 2 & 4 & 2 \\
\hline Peak voltage at switch & $1.67 \mathrm{kV}$ & $1 \mathrm{kV}$ & $2.1 \mathrm{kV}$ \\
\hline Peak current at switch & $0.55 \mathrm{kA}$ & $1.6625 \mathrm{kA}$ & $3.18 \mathrm{kA}$ \\
\hline Peak power at switch & $0.917 \mathrm{MW}$ & $1.6625 \mathrm{MW}$ & $6.67 \mathrm{MW}$ \\
\hline Average power at switch & $45.85 \mathrm{~kW}$ & $5 \mathrm{~kW}$ & $2.4 \mathrm{~kW}$ \\
\hline RMS current at switch & $123.1 \mathrm{~A}$ & $91.1 \mathrm{~A}$ & $60.3 \mathrm{~A}$ \\
\hline
\end{tabular}

Column 2 shows a possible design for the proposed femtosecond Xray source based on the same pulse transformer as described above. Compared to the other two examples its ratings are very conservative except for the average power together with the pulse repetition rate. The IGBT proposed for the SLAC project, part FZ80033KF1 from EUPEC, is rated for an RMS current of $800 \mathrm{~A}$ and should be able to provide the required $123 \mathrm{~A}$ with large reserve, supposing adequate cooling. Again the turn-off time with respect to the maximum obtainable pulse rate needs clarification with the manufacturer.

\section{Summary of pulsed rf gun systems}

The rf system for the photocathode rf gun is entirely feasible for $10 \mathrm{kHz}$ repetition rate and can be implemented with very small development risk. All three modulator concepts appear to be feasible by reasonable extrapolation of similar existing equipment. The maximum obtainable pulse rate is a critical parameter in all designs. A technical study is required to investigate the different possibilities in close contact with component manufacturers.

The most attractive concept is Variant $\mathrm{C}$, the induction modulator. It provides the reliability of low-voltage ground-referenced components, the flexibility of pulse length control and the convenience of a uniform modular structure. The superiority of semiconductor solutions may make the thyratron technology obsolete and lead to procurement problems in the long run.

Last but not least the cost may be a factor in the final decision. 


\section{REFERENCES}

[1] Koichiro Hirano et al., "High Power RF Test of the L-band CW Klystron," Paper presented at the $12^{\text {th }}$ Symposium on Accelerator Science and Technology, Waho, Japan 1999, pg 257..259.

[2] L.L. Reginato, LBNL, private communication.

[3] S.C.Glidden, "High voltage, high current, high di/dt solid state switch," Paper presented at the 4 Modulator Workshop MDK2001 in Geneva.

[4] A. Wellemann et al., "Semiconductors replace thyratrons and ignitrons," Paper presented at the 4 Modulator Workshop MDK2001 in Geneva.

[5] W.Crewson, "A new solid state High power pulsed modulator", Paper presented at the 4 Modulator Workshop MDK2001 in Geneva.

[6] R.L. Cassel et al., "Solid State Modulator Replacement for the Conventional SLAC 5045 Klystron modulator," XX Linac Conference Monterey 2000. 


\section{REFRIGERATION AND CRYOGENIC DISTRIBUTION SYSTEM}

This chapter describes the essential elements of the cryogenic system. The cryogenic distribution system starts at the level of the linac superconducting rf cavities [1] and moves out through the cryogenic piping to the liquid helium refrigeration plant that will be used to cool the rf cavities and the undulator magnets. For this report, the cryogenic distribution system and cryogenic refrigerator includes the following elements:

1) The piping within the linac cryogenic modules will influence the heat transfer through the super-fluid helium from the outer surface of the TESLA niobium cavity and the liquid to gas interface within the horizontal header pipe where the super-fluid helium boils. This piping determines the final design of the linac cryogenic module.

2) The supply and return piping dimensions are determined by the acceptable pressure drops.

3) The helium distribution system is determined by the need to cool down and warm up the various elements in the light source.

4) The size of the cryogenic plant is determined by the heat loads and the probable margin of error on those heat loads. Since the final heat loads are determined by the acceleration gradient in the cavities, a linac with five cryogenic modules will be compared to a linac with only four cryogenic modules.

The design assumes that all cryogenic elements in the facility will be cooled using a common cryogenic plant. To minimize vibration effects on the beamlines, this plant is assumed to be located some distance from the synchrotron light beamlines. All of the cryogenic elements in the facility will be attached to the helium refrigeration system through cryogenic transfer lines. The largest single cryogenic load is the main linac, which consists of four or five cryogenic modules depending on the design gradient for the cavities in the linac section. The second largest heat load comes from the cryogenic modules that contain the transverse deflecting rf cavities. The injector linac is the third largest heat load. The seven superconducting undulator magnets in the hard x-ray production section are the smallest heat loads connected to the light source refrigeration plant. The linac and deflecting cavity sections require helium cooling at $1.9 \mathrm{~K}, 5 \mathrm{~K}$ and $40 \mathrm{~K}$. The undulator magnets require two-phase helium cooling at 4.3 to $4.5 \mathrm{~K}$.

\section{TESLA CRYOGENiC MOdULE MODIFICATIONS}

The TESLA cavities have been demonstrated to operate routinely at gradients above $25 \mathrm{MV}$ per meter, and tests at the DESY test facility have shown operations above $35 \mathrm{MV}$ per meter in some cavities [1]. The proposed facility requires operating the superconducting cavities in $\mathrm{CW}$ mode to allow $10 \mathrm{kHz}$ bunch repetition rate, as opposed to the $0.7 \%$ duty factor of the TESLA design, resulting in significantly larger heat loads. A nine cell cavity operating in $\mathrm{CW}$ mode at $25 \mathrm{MV}$ per meter will have $63.5 \mathrm{~W}$ heat generated as a result of rf current flow on the inner surface of the cavity. Added to this is $8.5 \mathrm{~W}$ heat entering the cavity niobium body from the input rf power coupler. This dynamic heat load is to be transferred through the niobium to the cavity outer surface in the super-fluid helium liquid bath, then to the super-fluid helium surface where boiling occurs at $1.8 \mathrm{~K}$, without quenching the cavity. In a super-fluid helium test bath there is no problem transferring this heat from the cavity outer surface to the super-fluid helium surface, however, the transport of about $72 \mathrm{~W}$ from the cavity outer surface through the helium tank, the feed-pipe and the header-pipe configuration proposed for the TESLA cryomodules will require some modifications. 
With modifications to the helium tank, helium feed pipe and the helium header pipe the transport of heat within the TESLA cavity cryogenic module is not a problem. The heat transfer from the cavity surface to the liquid helium surface is limited by the heat transfer area in the tank (the area of between the cavity convolutions and the cavity helium tank wall), the total area of the helium feed pipe or pipes, and the area of the cross-sectional area of liquid helium that is in the feed pipe. In general, the heat transfer limit for the liquid super-fluid helium in the tank, the feed pipe and the header is about 0.6 to $0.7 \mathrm{~W} \mathrm{~cm}^{2}$ [2]. In TESLA, the area of the space between the cavity convolutions and the tank is about $40 \mathrm{~cm}^{2}$. The area of the feed pipe between the header and the tank is $28 \mathrm{~cm}^{2}$. The cross-sectional area of the liquid in a half-full header pipe is about $20 \mathrm{~cm}^{2}$.

In order to provide sufficient heat transfer from the cavity outer surface to the surface of the liquid in the header, the following modifications to the basic TESLA cryogenic module design are recommended:

1) The number of feed pipes between the rf cavity helium tank and the two-phase helium stand pipe should be increased from one to two.

2) The helium feeds should enter the helium tank at near the ends so heat can flow two ways along the rf cavity. If one puts the feeds at the one-quarter and three-quarter points along the cavity, the flow along the cavity is split four ways, and cavity cool down is not as effective.

3) The inside diameter of the helium tank should be increased by 10 to $15 \mathrm{~mm}$ to increase the spacing between the cavity convolutions and the cavity helium tank inner wall.

4) The liquid helium feed pipes from the stand-pipe to the tank should have their inside diameter increased from $60 \mathrm{~mm}$ to $100 \mathrm{~mm}$ (so that the effective inside area of the pipe is at least $78 \mathrm{~cm}^{2}$ ).

5) The two-phase helium header pipe should have its inside diameter increased from $70 \mathrm{~mm}$ to $100 \mathrm{~mm}$ to allow heat to flow through the liquid in a half full pipe.

If one does all of these things, the heat flow through the super-fluid helium in the tank and the pipes will less than $0.45 \mathrm{~W} \mathrm{~cm}^{2}$ in all of the liquid helium spaces. Figure 13-1 shows a crosssection view of the proposed modified TESLA cryogenic module. If the proposed changes do not fit into the standard TESLA cryogenic module cryostat vacuum vessel, the diameter of the 300-mm helium gas return pipe can be reduced to the next standard pipe size with an inside diameter greater than $250 \mathrm{~mm}$. 


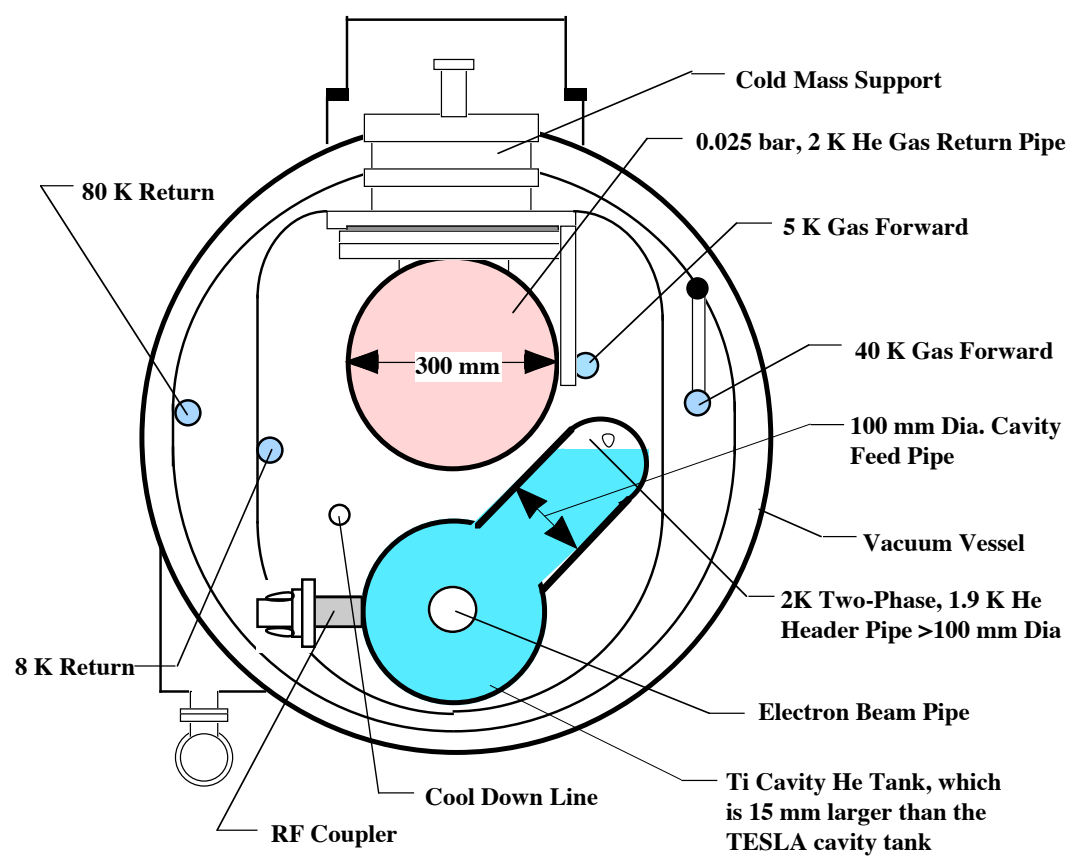

Figure 13-1 A cross-section of the proposed cryogenic module for the linac.

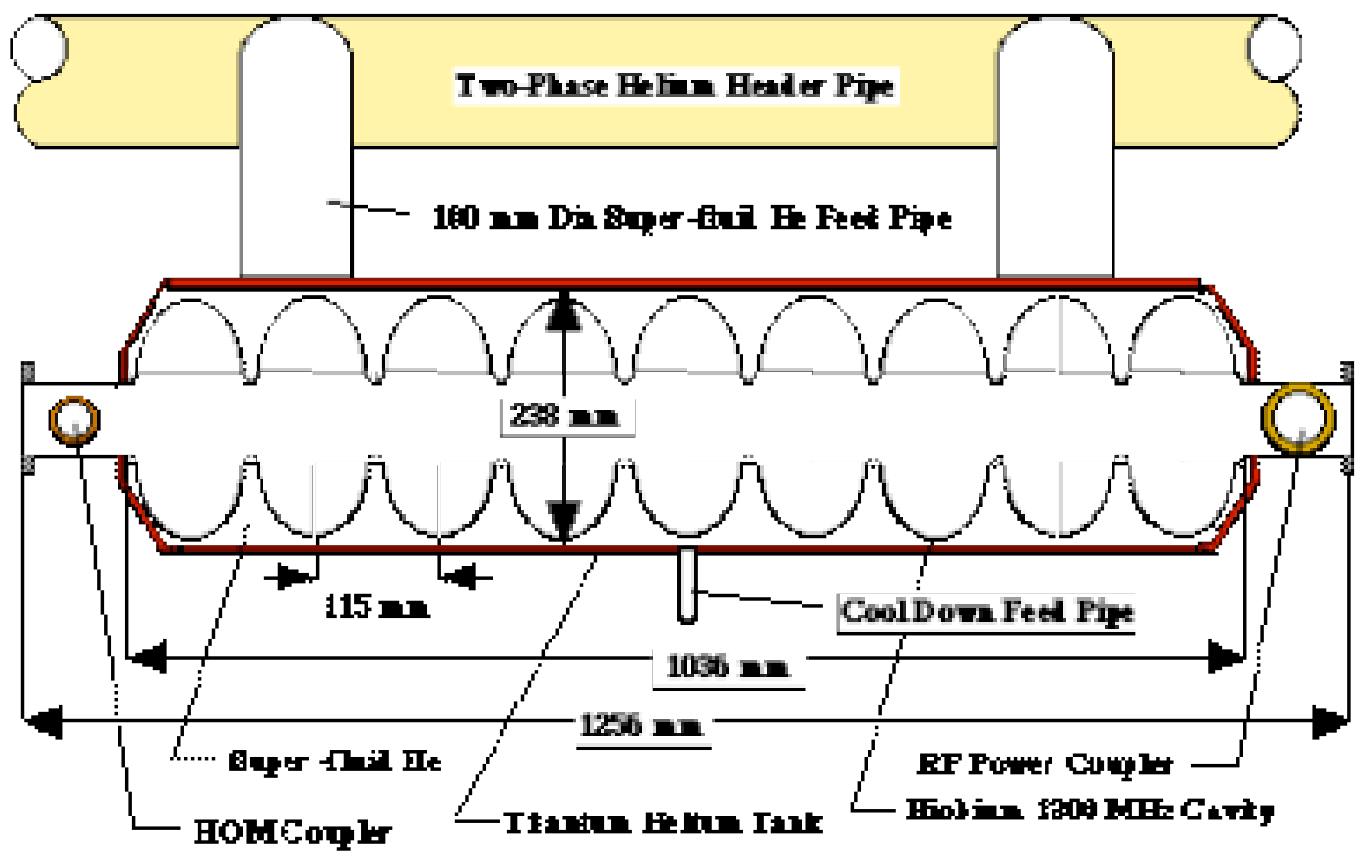

Figure 13-2 A Cross-section view of the increased diameter TESLA cavity helium tank, superfluid helium feed pipes, and the two-phase helium header. 


\section{CRYOGENIC MODUle PIPING AND TRANSFER LiNES TO THE COLD BOX}

As with the TESLA design, the linac cryogenic modules will be used as part of the helium transport system to and from the 1.8 to $1.9 \mathrm{~K}$ refrigerator (see Figures 13-1 and 13-2). The most important transfer line in the cryogenic module is the return line that carries $1.9 \mathrm{~K}$ helium back to the refrigerator cold box, since the pressure drop in this transfer line determines the operating temperature of the rf cavities. Helium gas in equilibrium with liquid helium at $2.0 \mathrm{~K}$ is at a pressure of $0.0313 \mathrm{bar}$ ( $23.5 \mathrm{torr})$. If the helium is at $1.8 \mathrm{~K}$, the saturation pressure drops to 0.0164 bar (12.3 torr). Helium in this range of temperature and pressure is an ideal gas with very low density, and as a result the helium volume flow through the return pipe must be high.

The pressure drop in the return line not only determines the pressure and temperature in the cavity helium tank, but it also determines the operating temperature of the cryogenic system cold box if the cavities operate below the lambda transition temperature of $2.176 \mathrm{~K}$. Since the excellent thermal conductivity of super-fluid helium is an integral part of removing the heat from the rf cavities, the operating temperature of the cavities can not be more than about $2.0 \mathrm{~K}$. Since the pressure drop along a pipe carrying gas in turbulent flow is proportional to the pipe length, proportional to the gas mass flow rate squared, and inversely proportional to the pipe inside diameter to the 4.8 power, we find that for given length of return line and mass flow one can get the desired pressure drop by increasing the pipe diameter. If the cavity operating temperature is set at $2.0 \mathrm{~K}$, a reduction of the return pipe size means that the cold box must operate at a lower temperature. A lower temperature cold box requires more input power to the compressors and is more expensive. In short, there is an optimum diameter for the $1.9 \mathrm{~K}$ return line to the cold box of about $250 \mathrm{~mm}$ for the proposed facility cryogenic system operating at $2 \mathrm{~K}$. (See Figure 13-3.) The TESLA design 300-mm return pipe shown in Figure 13-1 is just about the correct size. Because, the helium gas flow per TESLA cavity is increased by an order of magnitude, there must be frequent interconnect between the header pipe and the $1.9 \mathrm{~K}$ return.

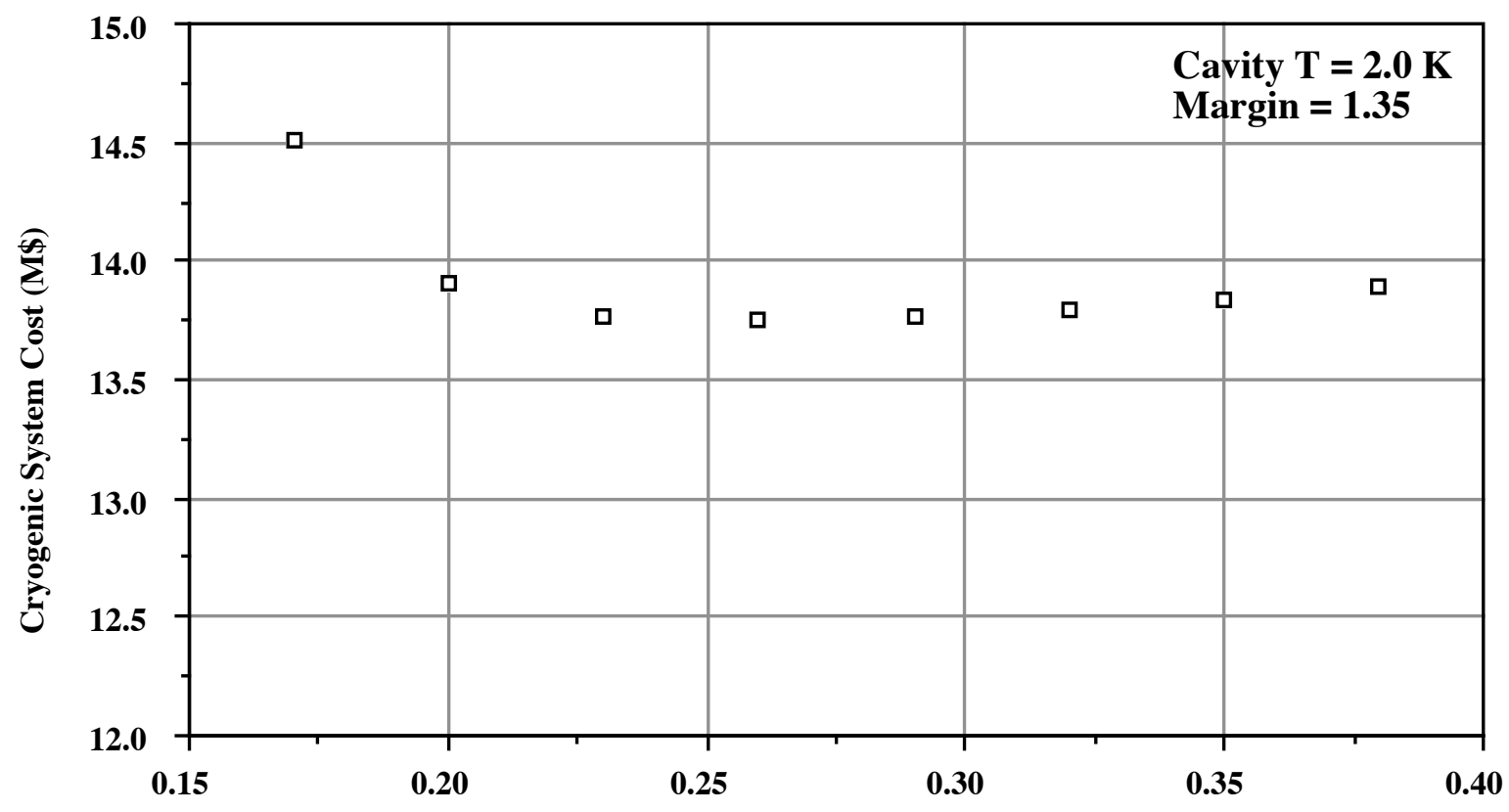

Return Pipe Diameter (m)

Figure 13-3 Refrigeration system cost versus the diameter of the $1.9 \mathrm{~K}$ helium return pipe. (The cold box is sized 35 percent larger than the calculated heat load.) 
The optimization of the return line has been performed for a number of cavity loads and refrigeration operating margins, and in no case has the optimum helium return line diameter been less than $240 \mathrm{~mm}$. As one can see from Figure 13-3, the optimum is rather broad. The 300-mm diameter pipe shown in Figure 13-1 seems to be a reasonable size that allows for extra line length and increased heat loads.

The two-phase helium header pipe shown in Figures 13-1 and 13-2 serves as the cryogenic supply line for the superconducting linac and is sized for heat transfer in the super-fluid helium. The pressure drop along this line during normal operation is quite low as long as the boil off gas is taken from the two-phase helium header and fed to the cold helium return pipe. This gas should be taken off at about every cavity in the cryogenic module. The header extends from one cryogenic module to the next, and the cold helium gas return pipe also extends down the length of the linac from one cryogenic module to the next.

The cool down pipe shown in Figure 13-2 is sized to allow the cavities to be cooled down quickly. Since dynamic (rf induced) loss is not a factor in this pipe size, the size used for the TESLA cryogenic modules is also adequate for our purposes. The cool-down pipe enters the bottom center of the cavity helium tank and the cool-down helium leaves the upper part of the tank through the super-fluid feed pipes, and is taken back to the cold box through the cold helium return pipe. During $2 \mathrm{~K}$, operation, the cool down line is filled with helium II.

Figure 13-4 shows a schematic representation of the flow circuit for the cryogenic modules of one of the rf sections. The circuit shown in Figure 13-4 applies for the main linac, the injection linac and the deflecting cavities. The line labeled the "cool down line" comes from lower end of the final expansion engine in the refrigerator cold box. The line labeled the "twophase helium header" also comes from the final expansion turbine. The gas in this line leaves the turbine, passes through a J-T heat exchanger and through a J-T valve. The other side of the $\mathrm{J}-\mathrm{T}$ heat exchanger is cooled by the $1.9 \mathrm{~K}$ gas returning from the rf cavities.

The flow circuits for the undulator magnets are different from the flow circuits for the rf cavity strings. The undulator magnets will be indirectly cooled using two-phase helium in tubes attached to the iron and coil package. The two-phase helium cooling system proposed is similar to the cooling system used for large detector magnets. The heat exchanger and phase separator causes the helium to enter the magnet string in the sub-cooled state. The magnets are electrically connected to the outside world through combined high temperature superconductor (HTS) leads and gas-cooled leads. The magnet shield and gas-cooled leads will be cooled by a helium stream from the $40 \mathrm{~K}$ circuit of the refrigerator cold box. This helium will pass through the shield then the gas-cooled leads returning to the compressor intake at $300 \mathrm{~K}$. An illustration of an undulator cooling circuit is shown in Figure 13-5. Note the top of the HTS leads is cooled from the gas in the cooled shield, the gas is then returned to the compressor intake at $300 \mathrm{~K}$. 


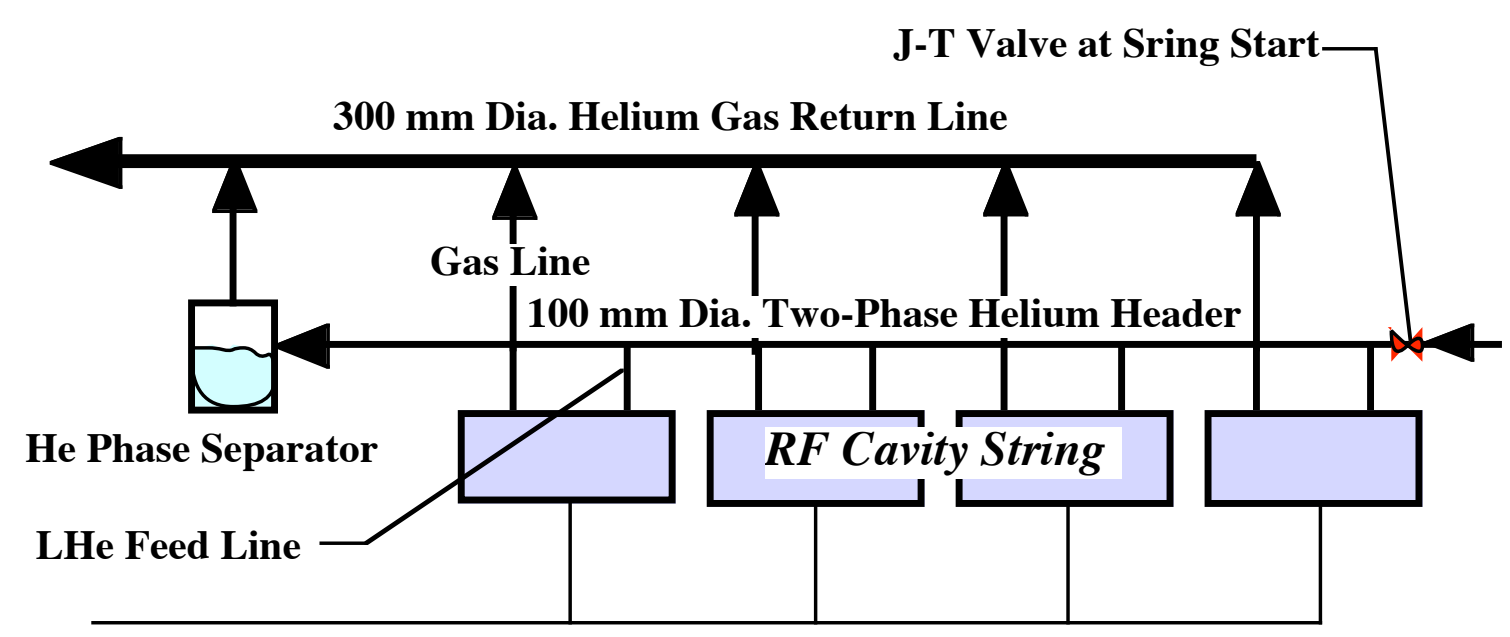

Cool Down Line

Figure 13-4 Simplified flow schematic diagram for the cooling of and the cool down of the rf Cavities. Note: the two-phase helium in the header comes from refrigerator cold box final J-T heat exchanger. The final J-T valve is shown at the start of the rf cavity string.

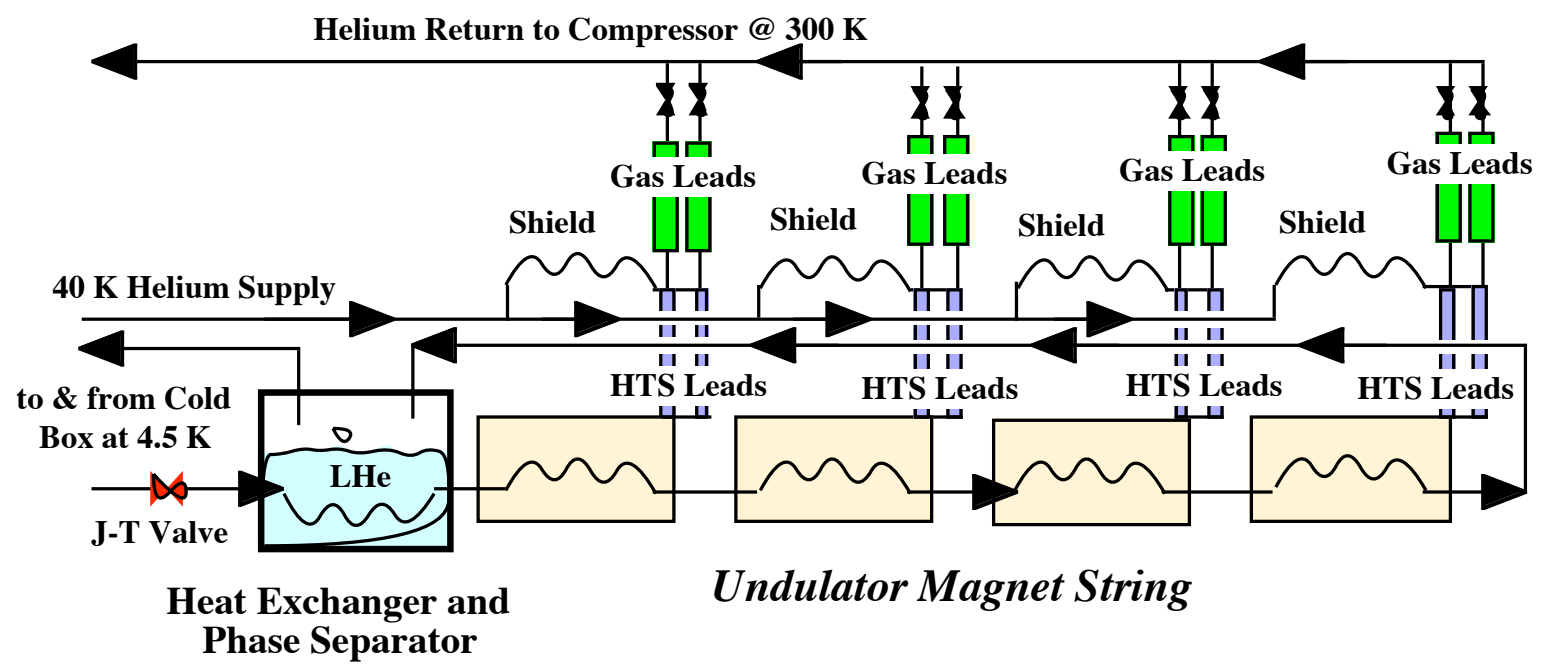

Figure 13-5 A flow schematic for the undulator magnet two-phase helium cooling system and the shield cooling. Note: Only four of the undulator magnets are shown. This circuit can be applied for many magnets in a string. 


\section{THE 1.9K HELIUM COLD BOX, WARM COMPRESSORS AND COLD COMPRESSORS}

A flow schematic for a typical $1.9 \mathrm{~K}$ cold box is shown in Figure 13-6. The cold box consists of a warm $(300 \mathrm{~K})$ compressor station, oil separation and inter-coolers, a series of helium heat exchangers (minimum of three pass heat exchangers), several stages of expansion engines, the final expansion valves to produce $1.9 \mathrm{~K}$ liquid helium (this valve is not shown in Figure 13-6, but it is shown in Figure 13-4.), and cold compressors to compress the helium at 0.0233 bar before it goes up the heat exchanger to the first stage compressor intake [3,4].

The compressor shown in Figure 13-6 is a three-stage compressor set, with low-pressure intake at 0.4 bar. The first intermediate pressure is at 1.4 bar; the second intermediate stage is at $5 \mathrm{bar}$; and the final stage is at 18 bar. The compression ratio of each stage is about 3.6. This ratio is quite typical for screw compressors used in modern helium refrigeration plants. Each stage of compression is followed by an inter-cooler to cool the helium to approximately $320 \mathrm{~K}$. After the final stage of compression an oil removal system is used to remove the oil that floods the compressors to improve cooling of the gas as it is compressed. The oil removal system and purifiers are not shown in Figure 13-6.

This arrangement of the expanders and heat exchangers is one that has been successfully used for $1.9 \mathrm{~K}$ systems. The configuration of a modern machine may, however, be somewhat different from that shown in Figure 13-6, in particular the refrigerator manufacturer will likely define the turbine and expander and heat exchanger configuration to best fits the type of turbine and the thermal dynamic cycle preferred. More efficient cycles will have more stages of optimized expansion, whereas cycles with fewer expansion stages and simpler heat exchanger configurations will less expensive to build. The final configuration of the refrigeration cycle will be set by the manufacturer during the proposal and bid process based on cost and the detailed machine design parameters.

A key element in modern $1.9 \mathrm{~K}$ systems is a cold compressor system that compresses the cold helium coming back through the J-T heat exchanger on the return side. The use of cold compressors reduces the size of the heat exchangers on the return side of the refrigerator cold box, and eliminates the low-pressure side of the heat exchanger pressure drop as a design factor. Early systems that operated without cold compressors were limited in low temperature capacity by this pressure drop. The cold compressors shown in Figure 13-7 are assumed to be about 65 percent efficient, large size cold compressors may be somewhat more efficient [5]. The use of cold compressors does not significantly improve the overall efficiency of the cycle, but they do reduce the capital cost of the cold box and warm compressor system. 


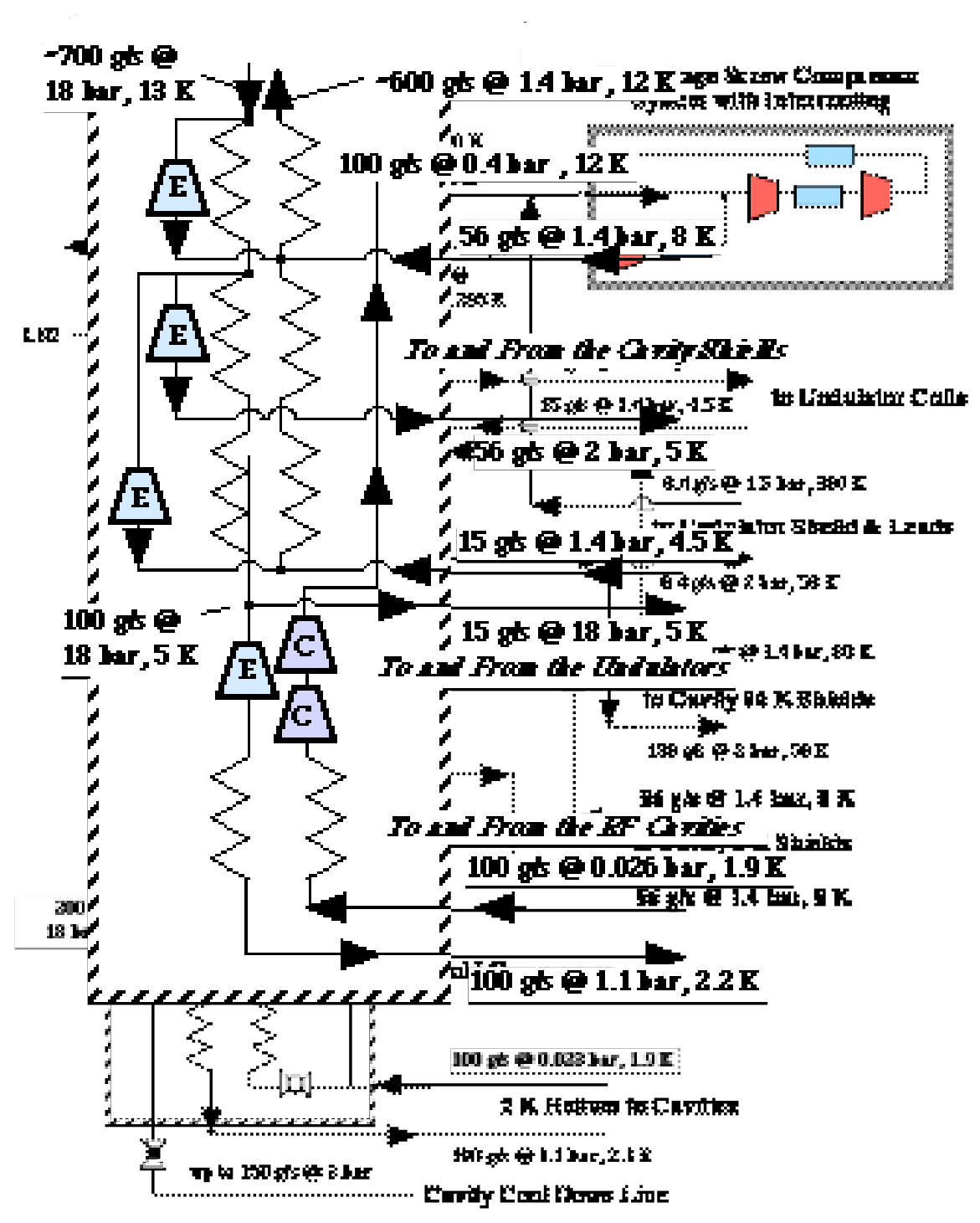

Figure 13-6 A flow schematic for the cold box and its warm and cold compressors. Note: the cold box used for the $1.9 \mathrm{~K}$ refrigerator may have more than three passes in the heat exchanges, and the compressor may have more stages than are shown. A more detailed view of the cold compressor end of the refrigerator cold box is shown in Figure 13-7. 


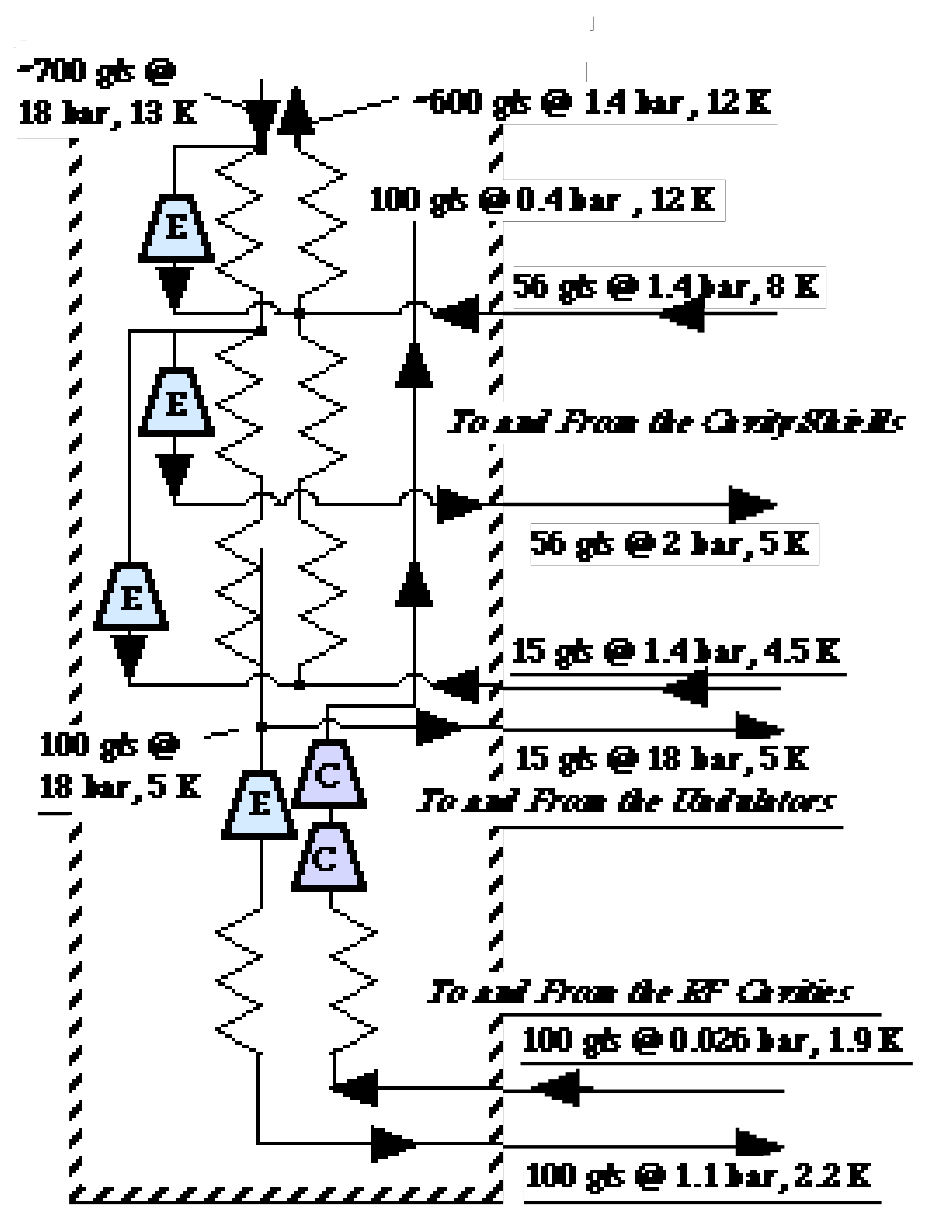

Figure 13-7 A flow schematic for the cold compressor end of a $1.9 \mathrm{~K}$ helium cold box. Note: The rest of the cold box is shown in Figure 13-6.

\section{CALCULATED HEAT LOADS AND REFRIGERATION REQUIREMENTS}

Table 13-1 shows the calculated heat loads for the cryogenic components. The injector linac is assumed to have one cryogenic module with an acceleration gradient of $15 \mathrm{MV}$ per meter. The cryogenic modules containing seven deflecting cavities are assumed to have a transverse gradient of $5 \mathrm{MV}$ per meter. There are seven 2-meter long undulator magnets that operate at 4.5 $\mathrm{K}$ in the hard $\mathrm{X}$-ray production section.

Two designs for the main linac are included in Table 13-1, the first assumes five cryogenic modules with eight cavities each operating at a design gradient of $20 \mathrm{MV}$ per meter, the second has four cryogenic modules with eight cavities each that operate with a design gradient of $25 \mathrm{MV}$ per meter. The higher gradient linac occupies less real estate, but the overall cost for that system might be higher. In all of the rf cavities the design $Q$ value was set at $10^{10}$, although the actual average cavity in the linac may have a higher $Q$ and this can dramatically reduce the dynamic loss at the cavity design operating temperature of $2.0 \mathrm{~K}$. Table 13-1 shows that $\mathrm{CW}$ operation of the rf systems results in heat loads at all temperatures that are dominated by dynamic losses. 
The second linac also has a lower operating margin for the cavity $Q$ and it has a higher temperature drop from the cavity wall to return gas. From tests of TESLA cavities at DESY, the average $Q$ of cavities operating at $25 \mathrm{MV}$ per meter is about $1.2 \times 10^{10}$. On the other hand, the average $Q$ of cavities operating at $20 \mathrm{MV}$ per meter is about $1.5 \times 10^{10}$. The average $Q$ for the cavities will have an effect on the operating margin (or design heat load) chosen for the refrigeration system used to cool the cavities. For the linac operating at $20 \mathrm{MV}$ per meter, the $2 \mathrm{~K}$ design heat load chosen is the same as the calculated heat load. For the linac operating at $25 \mathrm{MV}$ per meter, the $2 \mathrm{~K}$ design heat load chosen is 1.3 times the calculated heat load. For the pre-linac cavities and the deflecting cavities the design $2 \mathrm{~K}$ heat load is the calculated heat load. For heating at all other temperatures, the design heat load is set at 1.3 times the calculated.

Table 13-2 shows the design heat load for the entire machine at all cryogenic systems temperatures. For all cases, the design heat load is at least a factor of 1.3 higher than the calculated heat load. In the second and fourth columns of Table 13-2, the design heat load is converted to equivalent helium refrigeration at $4.5 \mathrm{~K}$. This allows one to calculate the size and cost of the helium plant needed. The refrigeration at $2 \mathrm{~K}$ is the largest fraction of the equivalent refrigeration at $4.5 \mathrm{~K}$, and has the largest influence on the cost of the overall refrigeration system. The cost of the refrigeration plant is proportional to the equivalent refrigeration at $4.5 \mathrm{~K}$ to the 0.7 power [6]. The cost of cryogenic system control, transfer lines, and compressor cooling is not strongly dependent on the equivalent design refrigeration at $4.5 \mathrm{~K}$. The cryogenic system cost with the shorter 25 MV per meter main linac is about $20 \%$ more expensive than the cryogenic system for the longer linac with a gradient of $20 \mathrm{MV}$ per meter.

Table 13-1 Calculated heat loads for various components

\begin{tabular}{|c|c|c|c|c|c|}
\hline Parameter & $\begin{array}{c}\text { Main } \\
\text { Cav }\end{array}$ & $\begin{array}{l}\text { linac } \\
\text { ities }\end{array}$ & $\begin{array}{l}\text { Injector } \\
\text { linac } \\
\text { Cavities }\end{array}$ & $\begin{array}{c}\text { Deflecting } \\
\text { Cavities }\end{array}$ & $\begin{array}{l}\text { Undulator } \\
\text { Magnets }\end{array}$ \\
\hline $\begin{array}{l}\text { Accelerating gradient }(\mathrm{MV} / \mathrm{m}) \\
\text { No. Cryogenic Modules } \\
\text { No. Cavities / Module }\end{array}$ & $\begin{array}{r}20 \\
5 \\
8\end{array}$ & $\begin{array}{r}25 \\
4 \\
8\end{array}$ & $\begin{array}{r}15 \\
1 \\
8\end{array}$ & $\begin{array}{l}5 \\
2 \\
7\end{array}$ & $\begin{array}{r}\text { NA } \\
7 \\
\text { NA }\end{array}$ \\
\hline $\begin{array}{l}\text { Heats Loads at } 2.0 \mathrm{~K} \\
\text { AC Losses }(\mathrm{W}) \\
\text { Static Heat Load }(\mathrm{W}) \\
\text { Total Heat Load }(\mathrm{W})\end{array}$ & $\begin{array}{r}1728.8 \\
32.2 \\
1761\end{array}$ & $\begin{array}{r}2139.6 \\
27 \\
2166.6\end{array}$ & $\begin{array}{r}198.6 \\
11.3 \\
209.9\end{array}$ & $\begin{array}{r}395.1 \\
15.4 \\
410.5\end{array}$ & $\begin{array}{l}-\cdots \\
- \\
-\end{array}$ \\
\hline $\begin{array}{l}\quad \text { Heat Loads at } 4.5 \mathrm{~K} \\
\text { AC Losses (W) } \\
\text { Static Heat Load (W) } \\
\text { Total Heat Load (W) }\end{array}$ & $\begin{array}{l}-- \\
- \\
- \\
-\end{array}$ & $\begin{array}{l}-- \\
-- \\
-\end{array}$ & $\begin{array}{l}-- \\
-- \\
--\end{array}$ & $\begin{array}{l}-- \\
-- \\
-\end{array}$ & $\begin{array}{r}0 \\
157.6 \\
157.6\end{array}$ \\
\hline $\begin{array}{l}\text { Heat Loads from } 5 K \text { to } 8 K \\
\text { AC Losses }(W) \\
\text { Static Heat Load }(\mathrm{W}) \\
\text { Total Heat Load }(\mathrm{W})\end{array}$ & $\begin{array}{r}394.4 \\
99.4 \\
493.8\end{array}$ & $\begin{array}{r}326.2 \\
81.9 \\
408.4\end{array}$ & $\begin{array}{r}75.5 \\
29.5 \\
105\end{array}$ & $\begin{array}{r}112.1 \\
43 \\
155.1\end{array}$ & $\begin{array}{l}- \\
-- \\
--\end{array}$ \\
\hline $\begin{array}{l}\text { Heat Loads from } 40 \mathrm{~K} \text { to } 80 \mathrm{~K} \\
\text { AC Losses }(\mathrm{W}) \\
\text { Static Heat Load }(\mathrm{W}) \\
\text { Total Heat Load }(\mathrm{W})\end{array}$ & $\begin{array}{r}15327.4 \\
500.2 \\
15827.6\end{array}$ & $\begin{array}{r}17918.5 \\
408.4 \\
18326.9\end{array}$ & $\begin{array}{r}1879.8 \\
132.1 \\
2011.9\end{array}$ & $\begin{array}{l}508.7 \\
202.4 \\
711.1\end{array}$ & $\begin{array}{r}0 \\
407 \\
407\end{array}$ \\
\hline
\end{tabular}


The input power to the cold box compressors is to first order proportional to the equivalent refrigeration at $4.5 \mathrm{~K}$. The longer linac is slightly more efficient than the smaller. In both cases, the efficiency assumed is about 22 percent of Carnot (without liquid nitrogen pre-cooling). Given the recent experience with machines at DESY and CERN one might expect actual machine efficiencies to approach 30 percent of Carnot. Roughly half of the cold box inefficiencies are in the helium compressors.

\section{CONCLUDING COMMENTS}

With modifications the TESLA cryogenic module can be used for $\mathrm{CW}$ operations at gradients up to $25 \mathrm{MV}$ per meter. It appears that the required modifications are feasible and fit within the existing TESLA design cryogenic module vacuum vessel.

Table 13-2 Design heat loads at various temperatures, the equivalent design refrigeration at $4.5 \mathrm{~K}$, and the input power needed to operate the cryogenic system.

\begin{tabular}{|l|r|r|r|r|}
\hline \multirow{2}{*}{} & \multicolumn{2}{|c|}{$20 \mathrm{MV}$ per $\mathrm{m}$} & \multicolumn{2}{c|}{$25 \mathrm{MV}$ per m } \\
\cline { 2 - 5 } & $\begin{array}{r}\text { Design } \\
\text { Load } \\
(\mathrm{W})\end{array}$ & $\begin{array}{r}\text { Equivalent } \\
\text { Refriger. } \\
\text { at 4.5 K } \\
(\mathrm{kW})\end{array}$ & $\begin{array}{c}\text { Design } \\
\text { Load }\end{array}$ & $\begin{array}{r}\text { Equivalent } \\
\text { Refriger. } \\
\text { at 4.5 K } \\
(\mathrm{kW})\end{array}$ \\
\hline Heat \& Refrigeration at 2.0 K & 2381.4 & 5.5 & 3437 & 7.99 \\
Heat \& Refrigeration at 4.5 K & 157.1 & 0.16 & 157.1 & 0.16 \\
Heat \& Refrigeration at 5 to 8 K & 980 & 0.88 & 868.7 & 0.78 \\
Heat \& Refrigeration at 40 to 80 K & 24522.7 & 2.43 & 27771.4 & 2.75 \\
Total Equivalent 4.5 K Refrigeration & & 8.97 & & 11.68 \\
Input Power for the Cryogenic System & & $3.4 \mathrm{MW}$ & & $4.3 \mathrm{MW}$ \\
Minimum Installed Cooling Capacity & & $3.6 \mathrm{MW}$ & & $4.5 \mathrm{MW}$ \\
\hline
\end{tabular}

The refrigeration plant needed to cool the cavities and undulator magnets is similar in size to one of the three existing HERA cryogenic plants at DESY. The technology for such plants is well understood and there are at least two reliable vendors who can produce the cryogenic plant. The rest of the cryogenic system is understood well enough to allow a cost estimate to be made. The cryogenic system also fits within the parameter space of cryogenic systems that have been built at other locations.

The choice of acceleration gradient in the linac cavities does have an impact on the cost and the operating margin for the cryogenics system, and it appears that a lower acceleration gradient linac may be the more cost effective option. 


\section{Acknowledgements}

The design team wishes to thank D. Proch, S. Wolff, and B. Peterson of DESY for their candid comments concerning the superconducting rf cavity cooling system for CW operations.

\section{REFERENCES}

[1] “TESLA Technical Design Report,” DESY 20010011, March 2001

[2] S. Wolff, DESY, private commination July 2002

[3] J. L. Smith Jr. and G. Robinson, "A Tribute to Samuel C. Collins: September 28, 1898 June 19, 1984," Advances in Cryogenic Engineering 31, p 1, Plenum Press, New York (1985). On page 6 , this paper references the first closed cycle $1.8 \mathrm{~K}$ helium refrigerator ever built by Sam Collins. The report refers to a cold compressor designed by Sam Collins in 1967. The notes were never published and the compressor was never built.

[4] G. M. Gistau, G. Claudet, "The Tore Supra 300 W - 1.75 K Refrigerator," Advances in Cryogenic Engineering 31, p 607, Plenum Press, New York, (1985)

[5] J. Fuerst, "Selection of Cold Compressors for the Fermilab Tevetron," Advances in Cryogenic Engineering 39, p 863, Plenum Press, New York (1993)

[6] R. A. Byrns and M. A. Green, "An Update on Estimating the Cost of Cryogenic Refrigeration," co-authored R. Byrns, Advances in Cryogenic Engineering 43, p 1661, Plenum Press, (1997), LBNL-40188 


\section{MAGNET DESIGNS}

\section{INTRODUCTION}

The lattice consists of conventional dipole, quadrupole and sextupole magnets constructed with hollow conductor water cooled windings and laminated iron pole and yokes. The dipole magnets within the arc sections of each of the four rings are identical rectangular " $\mathrm{C}$ " type. Curved magnets are planned for the transition arc from the injector to the main linac. The quadrupole and sextupole magnets are variations on magnets designed and built for the ALS booster ring. To allow for future modifications and design maturation, the magnet designs incorporate a $150 \%$ margin on beam energy [1].

The magnet system employs the most mature of technologies associated with accelerators. Despite this, a few of the magnets in the lattice demand relatively sophisticated design and engineering. Other magnets within the lattice can be extrapolated from existing (proven) designs. The spatial constraints as well as the need to allow for future upgrades require that the designs be highly evolved and that the parameters be narrowly defined.

\section{REQUIREMENTS}

The accelerator baseline design includes four electron rings with beam energies of approximately $0.7,1.3,1.9$ and $2.5 \mathrm{GeV}$. All four beams are accelerated through the same linac and the different energy beams are separated in the horizontal plane before they orbit through their respective rings. The most demanding magnet designs are those in the separator and combiner sections where the separation between beamlines is small resulting in requirements for septum styled dipoles and quadrupoles (see Figure 14-1). The leakage field from the septum magnets to the adjacent beamlines must be quantified and reduced. An upgrade to a final energy of $3.1 \mathrm{GeV}$ is anticipated but this falls well within the $150 \%$ margin applied to the baseline final energy.

Although the fields and gradients of the various magnet types are modest for the baseline beam energy, in addition to having to deal with pole and yoke saturation effects, the $150 \%$ field margin represents a $>225 \%$ power increase with similar demands on the cooling circuits. The ability to operate at $150 \%$ of the baseline energy requires a more complete and detailed magnet design than the "generic" geometries typically used in zero-order conceptual designs. Consequently, the magnet designs presented in this document reflect a greater amount of engineering than is typical of a concept at this point of development.

The total power requirement for all lattice magnets for the facility is approximately $2 \mathrm{MW}$. Details of coil package and magnet power supply requirements are to be found in the references at the end of this chapter. 


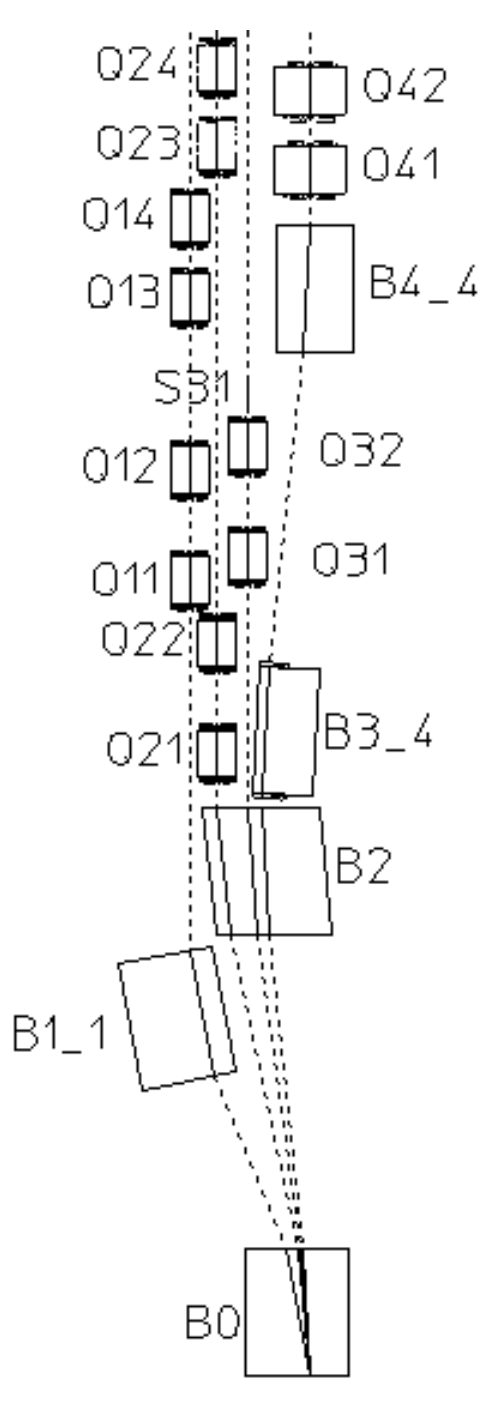

Figure 14-1 Magnets in a beam spreader region. The linac is at the lower end of the diagram, and the four rings continue at the upper end.

\section{Field Quality}

At present, the field quality requirements are not completely specified for each magnet type. Therefore, field quality requirements for magnets employed in a typical third generation synchrotron radiation storage ring have been used as achievable goals. This should be adequate since the stringent field quality requirements for storage rings with a beam lifetime of many hours are typically more demanding than for most other accelerators. 


\section{SEPTUM DIPOLE (B3_4)}

The septum dipole, shown in Figure 14-2, is the most demanding of the magnets [2]. The limited space between the 1.9 and $2.5 \mathrm{GeV}$ beamlines requires a high current density in the septum. At $150 \%$ the nominal energy, the water circuit topology requires one water circuit per turn. Detailed POISSON calculations have been performed to evaluate both the field uniformity in the gap as well as the leakage field in the adjacent beamline with an iron shield inserted in the narrow 4-mm space between the magnet and the $1.9 \mathrm{GeV}$ beamline.

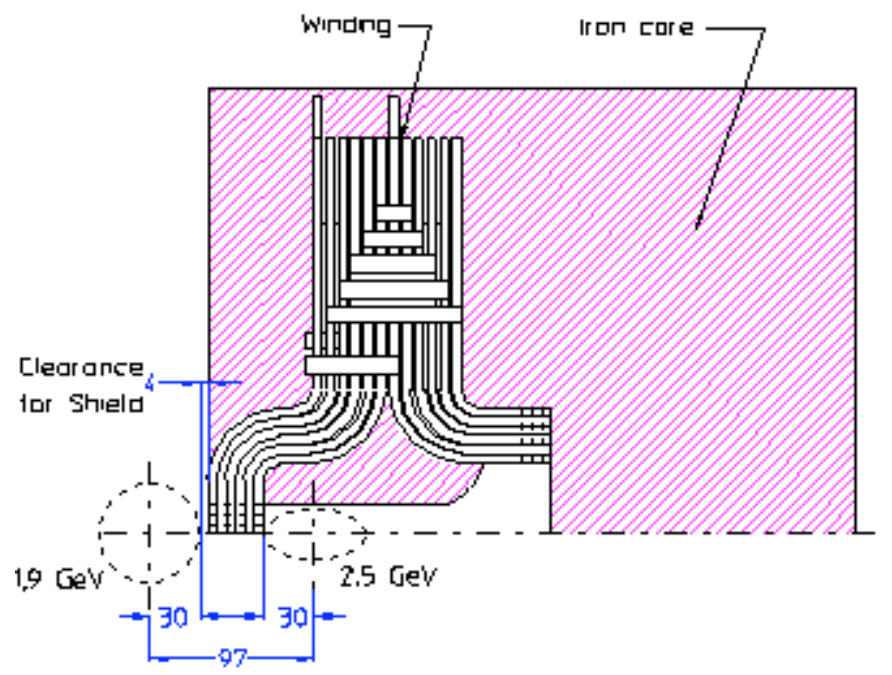

Figure 14-2 Septum Dipole Magnet.

\section{SEPTUM QUADRUPOLE}

The septum quadrupole used in the combiner and spreader sections, shown in Figures 14-3 and 14-4, employs a figure-8 design [3]. The 7.64- $\mathrm{mm}$ space between the edge of the magnet and the adjacent beamline will be utilized for magnetic shielding, although the narrow vertical leg provide some shielding. Since magnet requirements vary widely, two variations are used, one with half the number of windings than the other. 


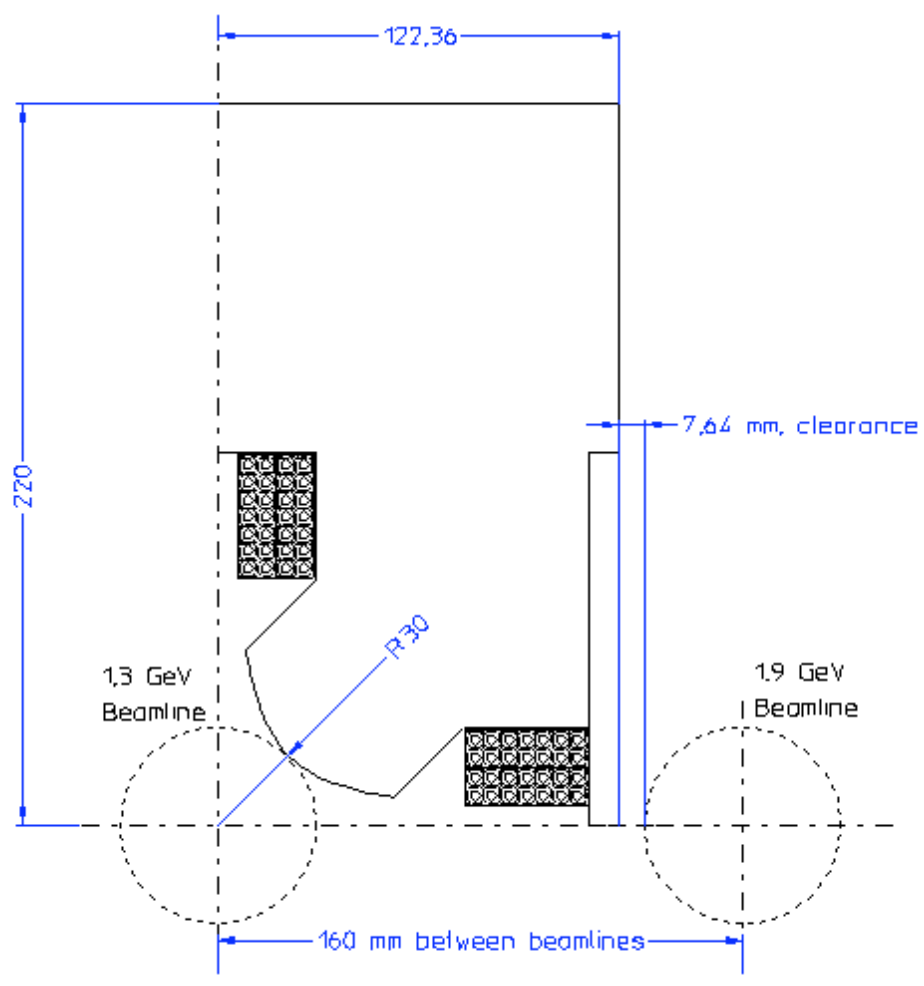

Figure 14-3 One quadrant of a septum quadrupole magnet in cross section.

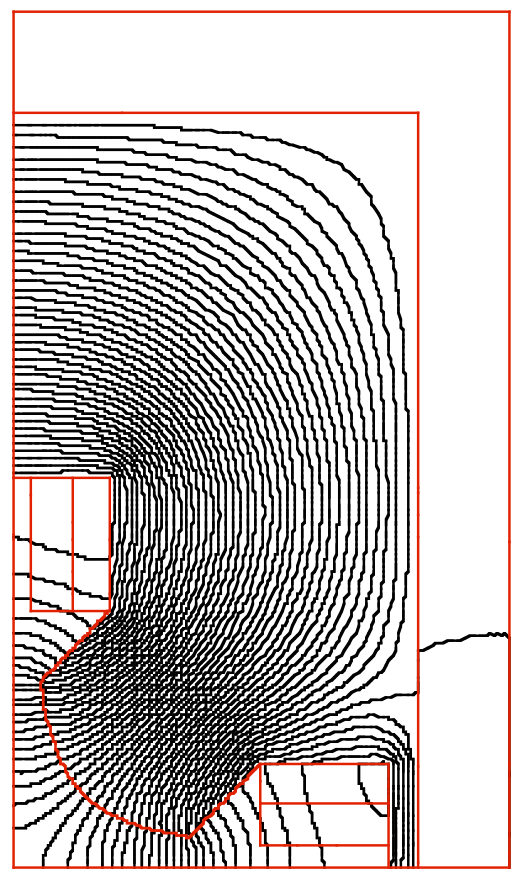

Figure 14-4 Plot of magnetic field lines for septum quadrupole. 


\section{MAIN Ring BENDS}

Longitudinal and transverse spatial constraints result in a tall coil package and a narrow pole $[4,5]$. To meet a large dynamic range requirement, good field uniformity needs to be maintained from $<1 \mathrm{~T}$ to $>1.5 \mathrm{~T}$.

A layout of the lattice at the entrance to the arc sections is shown in Figure 14-5. The baseline geometry is constrained by the $17.5 \mathrm{~mm}$ half sagitta for the softest $(0.7 \mathrm{GeV})$ beam. The $15-\mathrm{mm}$ half aperture, including a reasonable pole overhang to assure good field quality, results in the pre-optimized pole geometry shown in Figure 14-6. The coil shape has been determined by requiring a reasonable current density to minimize power consumption. This results in a fairly steep pole-edge angle.

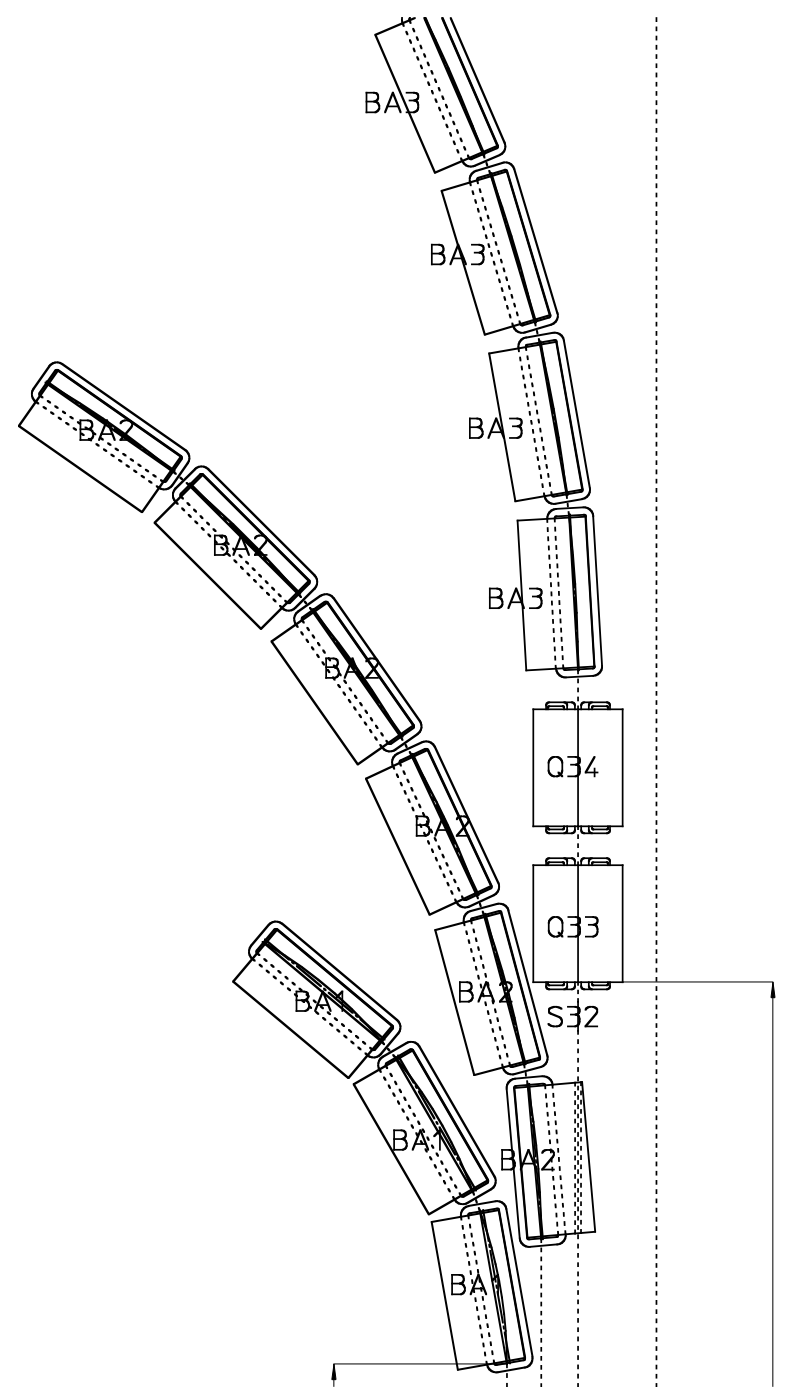

Figure 14-5 Magnet layout at the entrance to the ring arc sections. Bend magnets in the arcs are denoted $B A$. 


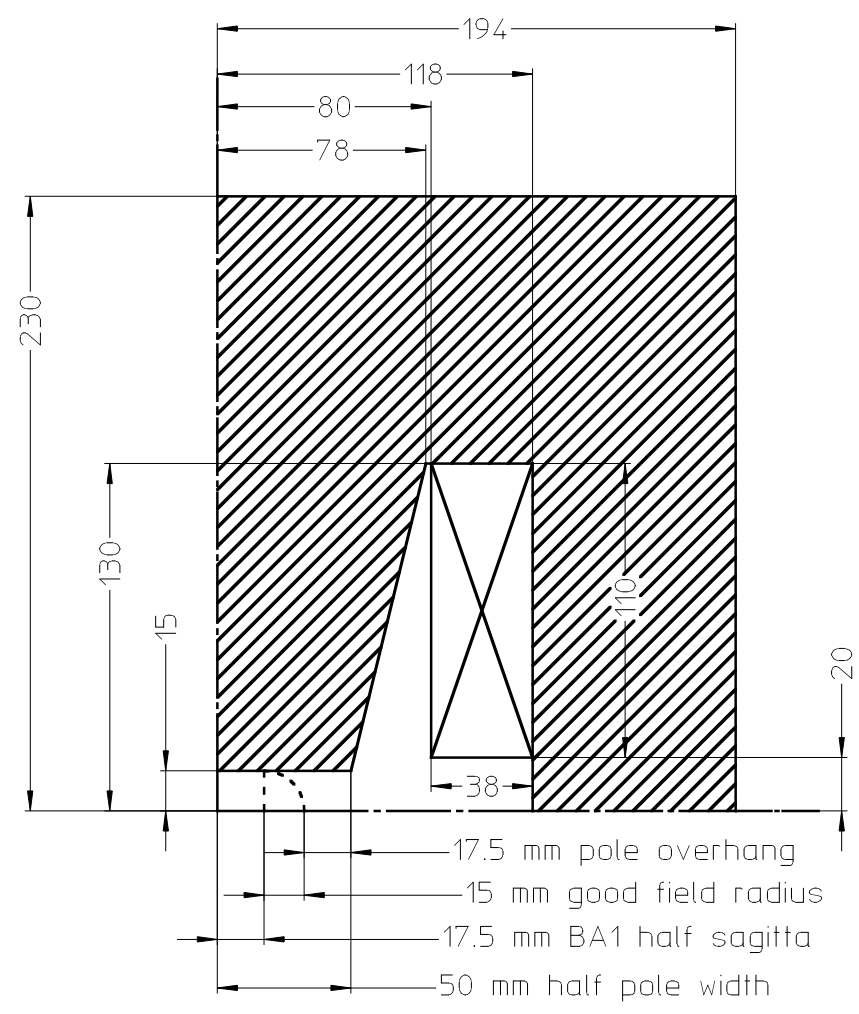

Figure 14-6 Starting geometry for bend magnet pole optimization.

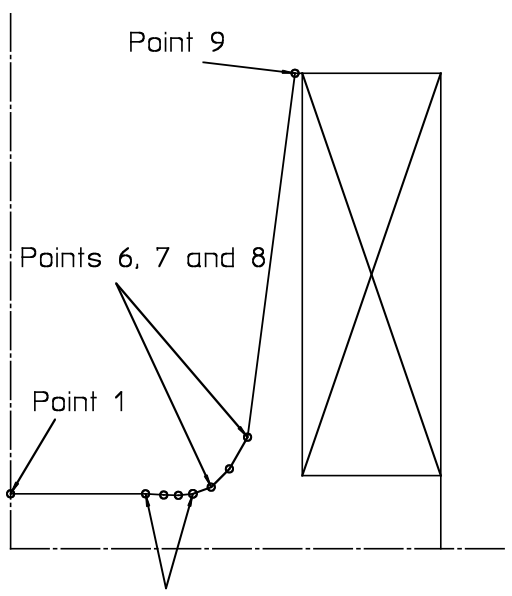

\begin{tabular}{|c|c|c|}
\hline Points & $\mathrm{X}(\mathrm{mm})$ & $\mathrm{Y}(\mathrm{mm})$ \\
\hline 1 & 0 & 15 \\
\hline 2 & 37 & 15 \\
\hline 3 & 42 & 14.7 \\
\hline 4 & 46 & 14.6 \\
\hline 5 & 50 & 15 \\
\hline 6 & 55 & 16.82 \\
\hline 7 & 60 & 21.82 \\
\hline 8 & 65 & 30.48 \\
\hline 9 & 78 & 130 \\
\hline
\end{tabular}

Points 2, 3, 4 and 5

Figure 14-7 Dimensions of an optimized arc bend magnet pole.

We have placed a radius on the pole corner in order to maintain field uniformity throughout the full range of excitation (a sharp pole corner tends to disappear as it saturates at high field). Finally, amplitudes of selected points on the pole were adjusted until good field uniformity is achieved at both low and high excitations. 


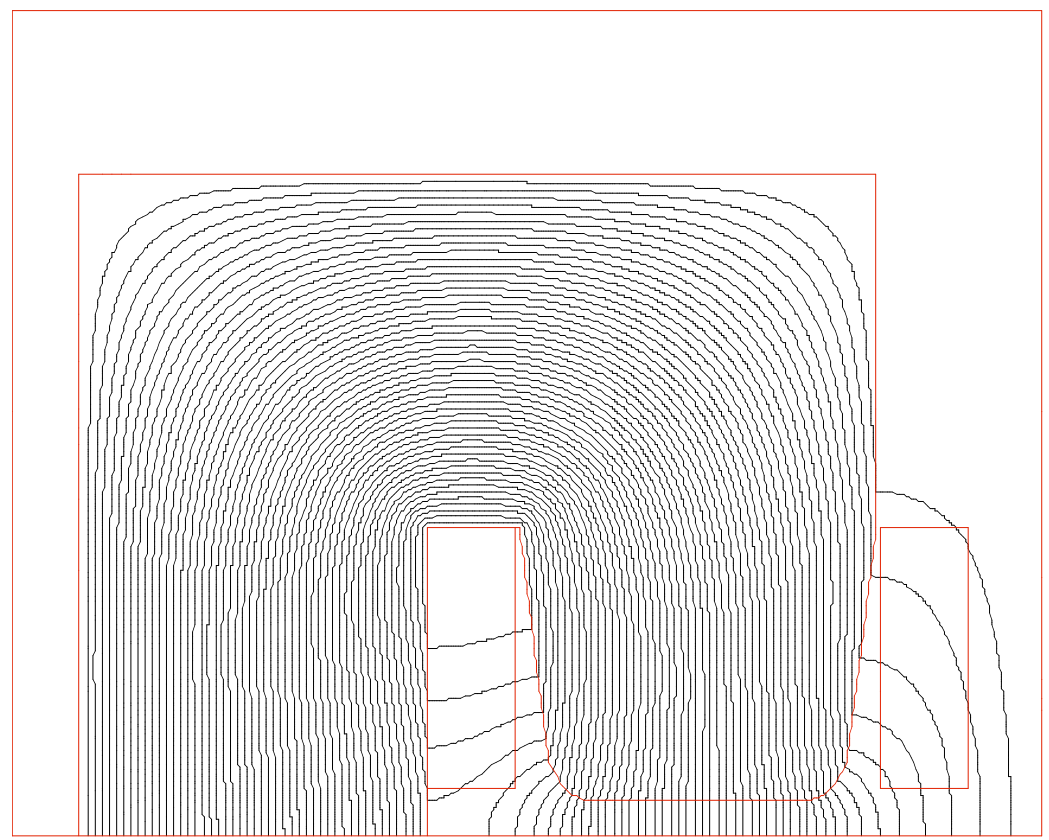

Figure 14-8 Magnetic field plot for an arc dipole magnet after pole shape optimization.

\section{Main Ring Quadrupoles}

The yoke design for the main ring quadrupoles is patterned after the ALS booster and booster to storage ring quadrupoles [6]. It is anticipated that the same die set used to stamp the laminations for these magnets can again be used. The coil design is patterned after the storage ring quadrupole coils, using small conductors so that low-current power supplies may be used.

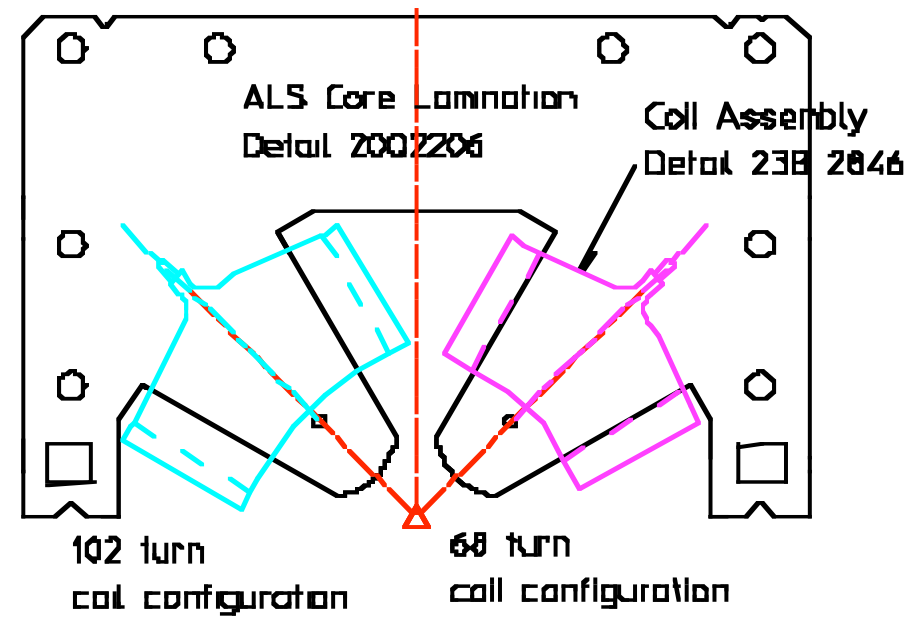

Figure 14-9 Yoke and coil configuration of the quadrupoles for the ALS booster. 


\section{Main Ring SeXtupoles}

The sextupole core laminations use the same shape as that of the ALS booster sextupoles [7]. The 32-turn coils are wound using hollow conductor and are tall and narrow to fit between adjacent poles. Figures 14-10 and 14-11 show the cross-sectional (half) view and a magnetic field plot of a $1 / 12$ segment.

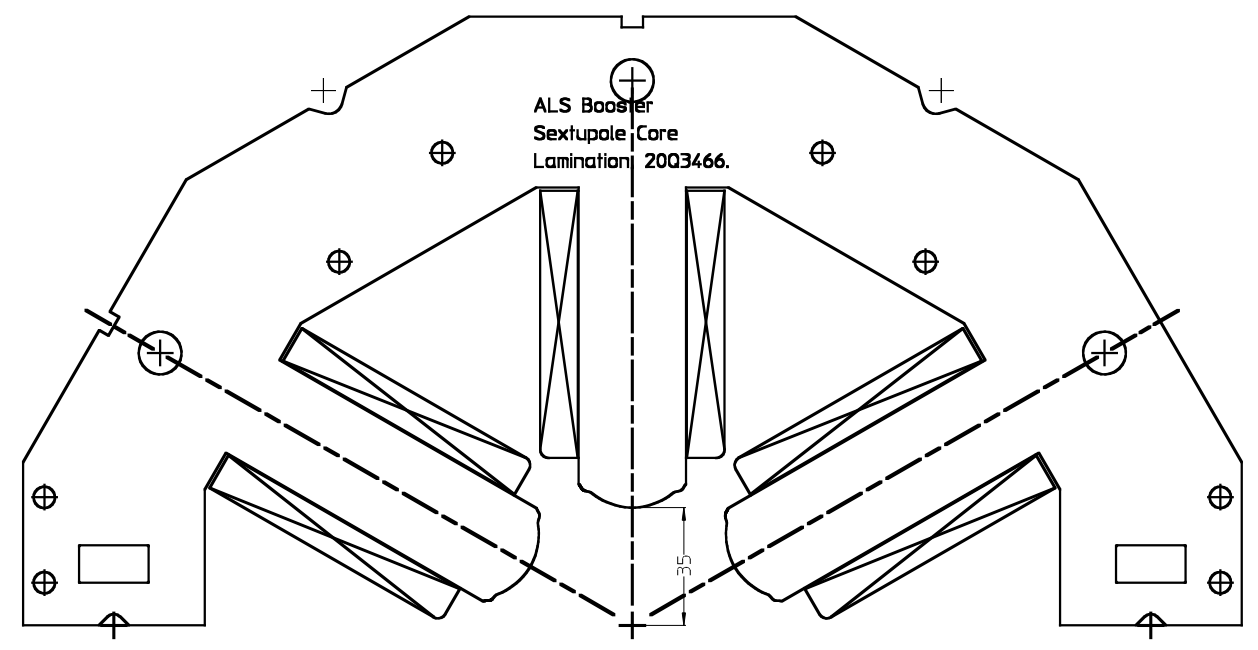

Figure 14-10 Cross-section of a lamination of the ALS sextupole design.

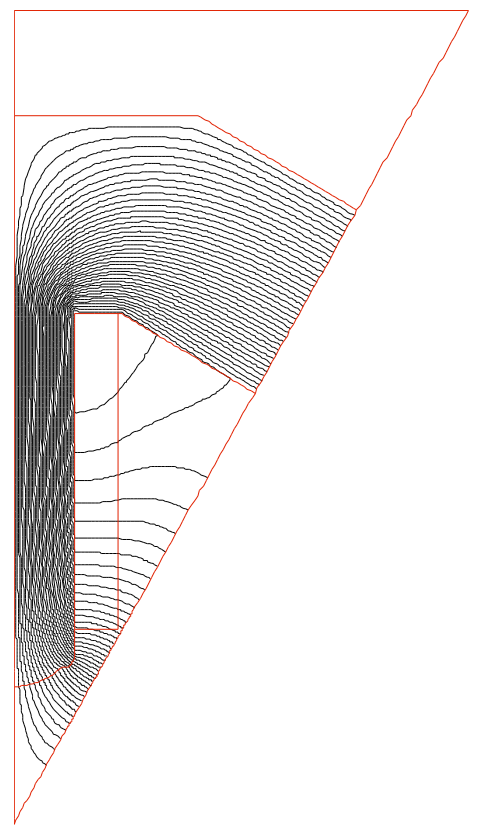

Figure 14-11 Magnetic field plot of ALS 1/12 sextupole magnet. 


\section{REFERENCES}

[1] J. Tanabe, "Recirculating linac magnet designs summary", LBNL Eng. Note M8153, November 2001.

[2] J. Tanabe, "Recirculating linac spreader magnet designs", LBNL Eng. Note M8151, November 2001.

[3] J. Tanabe, "Recirculating linac septum quadrupole designs", LBNL Eng. Note M8157, November 2001.

[4] J. Tanabe, "Recirculating linac spreader main bend magnet coil designs", LBNL Eng. Note M8154, November 2001.

[5] J. Tanabe, "Recirculating linac main bend core and pole designs", LBNL Eng. Note M8155, November 2001.

[6] J. Tanabe, "Recirculating linac main bend core and pole designs", LBNL Eng. Note M8156, November 2001.

[7] J. Tanabe, "Recirculating linac main sextupole design", LBNL Eng. Note M8152, November 2001. 


\section{VACUUM SYSTEMS}

The vacuum system for the proposed facility is readily achievable with conventional vacuum techniques, hardware, and established fabrication processes. Many of the difficult technical challenges associated with synchrotron light source storage rings (such as ALS, NSLS, etc.) are sidestepped by the relatively short beam lifetime of the recirculating linac. The short beam lifetime allows relatively high limits on background gas pressure. The one exception to this is in the region of the photocathode where vacuum pressures of $10^{-11}$ are often required to preserve cathode lifetimes. The low-average beam current also contributes to the simplification of the vacuum system design. As will be explained in more detail, the $10-30 \square \mathrm{A}$ beam current produces very little synchrotron radiation power loading on the vacuum chamber walls and thus the need for an ante-chamber (as used in ALS, PEP-II and other machines) is eliminated. With very modest limits on the gas pressure and minimal outgassing due to low levels of synchrotron radiation, the vacuum chamber dimensions can be adjusted to minimize the energy loss due to coherent synchrotron radiation (CSR) and resistive wall wakefield effects.

For significantly increased beam power levels, a more standard vacuum chamber with distributed ion pumps within the dipole magnets, and perhaps a separately pumped ante-chamber may be desirable. The proposed machine configuration does not preclude this as a potential upgrade.

The baseline electron beam current is $10 \square \mathrm{A}(1 \mathrm{nC} / \mathrm{bunch}$ at $10 \mathrm{kHz})$ at an energy of $2.5 \mathrm{GeV}$. However, to allow for potential upgrades to the design, the vacuum system is designed for $30 \square \mathrm{A}$ at $3.1 \mathrm{GeV}$. The bunch length is compressed from $20 \mathrm{ps}$ at the cathode to $2 \mathrm{ps}$ at the exit of the arc connecting the pre-accelerator to the re-circulating linac (Arc 0). This bunch length is then held constant throughout the re-circulating sections. Note the term Arc as used throughout this chapters refers to entire circulation path at a given energy, straight sections as well as the curved sections at the ends of the straights.

The design of vacuum systems for synchrotron radiation sources must consider several physical processes in order to establish the allowable background gas partial pressures and chamber dimensions. These processes include:

- Beam loss due to interaction with background gas by

$\circ$ inelastic (Bremsstrahlung) scattering of the beam with residual gas nuclei and

- large angle elastic (Coulomb) scattering of the beam with residual gas nuclei.

- Energy loss, resulting in longitudinal emittance growth due to coherent synchrotron radiation (CSR)

- Transverse emittance growth due to resistive wall wakefields

To reduce the energy loss resulting from CSR, the vacuum chamber aperture is reduced as reported in Chapter 4-Collective Effects. This has the effect of producing a higher cut-off frequency for the radiation propagating within the vacuum chamber. The coherent radiation emission process is then effectively suppressed. An upper limit on the vacuum chamber internal height (or equivalently the amount of CSR shielding required) is determined by allowed energy 
loss. The CSR affect is present only in the bend magnets, and a larger beampipe aperture may be employed elsewhere.

The resistive wall wakefield is generated by the electron beam traveling in a vacuum chamber of finite conductivity. It is proportional to the chamber length and inversely proportional to both the square root of the chamber's electrical conductivity $\square_{c}$ and the cube of the chamber's half-height, $b$. The resistive wall effect therefore has implications on the choice of vacuum chamber material, the minimum tolerable chamber height, and the location tolerance of the chamber with respect to the nominal beam axis.

The final chamber geometry, and most importantly the chamber inner height, will be determined by selecting an appropriate trade off between reducing the effects of CSR, resistive wall, and beam loss due to collisional scattering with the background gas. An example of this trade is the plot of the CSR energy loss and transverse resistive wall distortion as a function of chamber height shown in Figure 15-1. These affects are different for each beam pass as a result of the different beam energy and magnet bend radii. The optimal chamber height is therefore different for each arc. From an analysis of collective effects, as described in Chapter 4-Collective Effects, a minimum vacuum chamber height of $9 \mathrm{~mm}$ is indicated.

Since the actual values and effects of longitudinal emittance growth are still to be assessed, the beam chamber heights indicated in this report are preliminary.

\section{Beam Loss Due to BeAM - Gas Collisions}

In contrast to storage rings, the $10 \square \mathrm{s}$ time-of-flight (900 meter total path length) place extremely modest limitations on the allowable background gas pressure. We consider here in elastic and elastic scattering. 


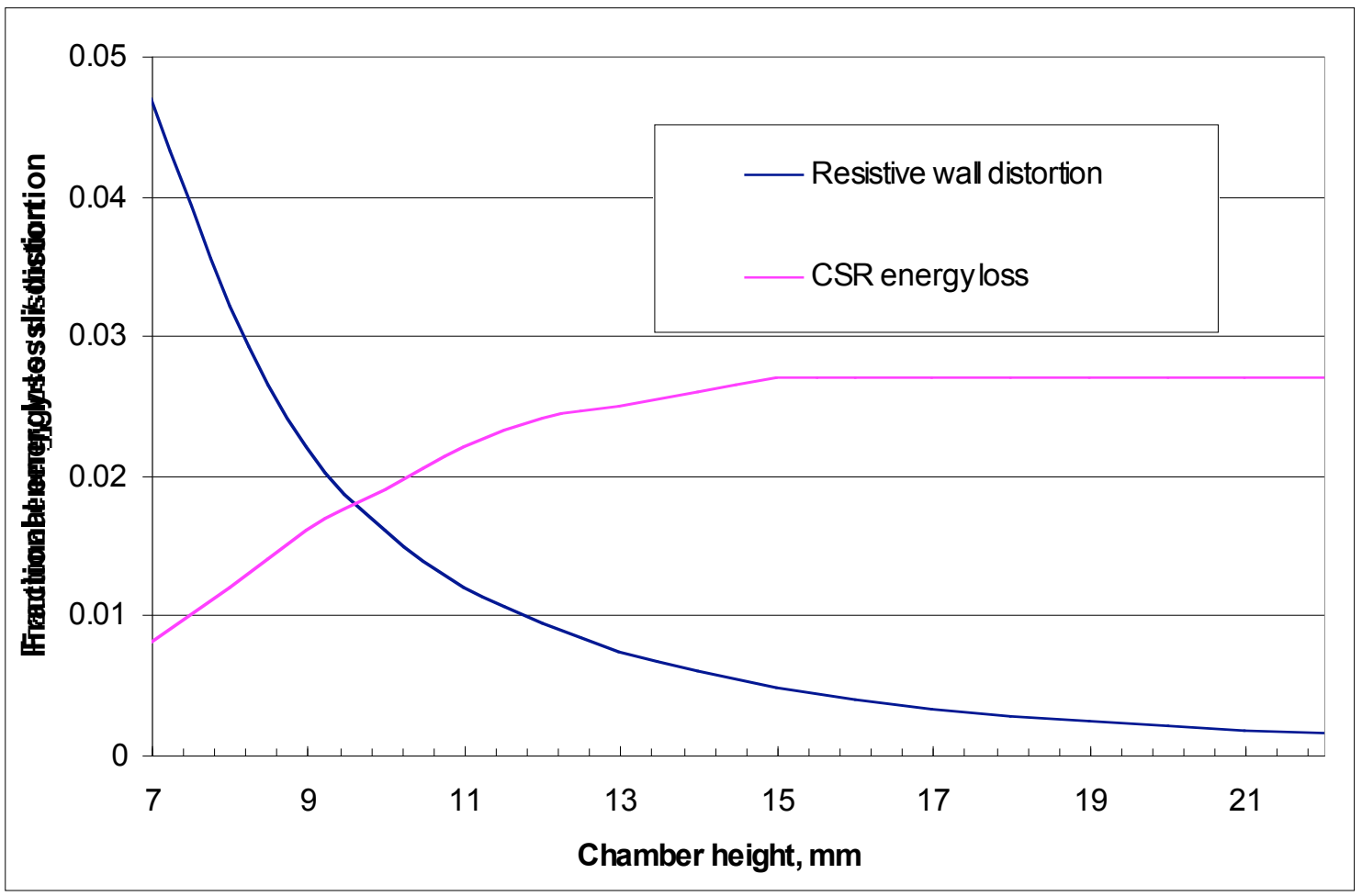

Figure 15-1 Comparison of fractional energy loss due to CSR and beam distortion from resistive wall effects as a function of chamber height.

\section{Inelastic Scattering}

Charged particles passing through matter become deflected by the strong electric fields of the nucleus. This deflection constitutes a transverse acceleration of the particle and results in a loss of energy through Bremsstrahlung radiation. If the energy loss exceeds the energy acceptance of the accelerator, $\square_{\mathrm{acc}}$, $\left(\square_{\mathrm{acc}}=\square \square \square\right.$ the particle is lost. For highly relativistic beams the probability for a particle to suffer a fractional energy loss of more than $\bigsqcup_{\text {acc }}$ per radiation length is [1],

$$
P_{r}=\square \frac{4}{3} \ln \left(\square_{a c c}\right)
$$

The probability of particle loss per unit time is equal to the beam decay rate or equal to the inverse of the bremsstrahlung lifetime,

$$
\square_{b s}^{\square 1}=\square \frac{4}{3} \frac{c}{L_{r}} \ln \left(1 / \square_{a c c}\right)
$$

where $c$ is the speed of light and $L_{r}$ is the radiation length. The radiation length for gas is a function of the partial pressure of each residual gas species. However, the composite atomic number of typical gas components in ultra high vacuum systems is roughly equivalent to 
nitrogen. Therefore the radiation length for nitrogen $(200 \mathrm{~m}$ at standard temperature and pressure (STP)), scaled appropriately for pressure, $\mathrm{P}_{\text {vac }}$ [nTorr], has been used to derive a simplified equation for bremstahlung lifetime, [1]

$$
\Gamma_{b s}^{11}\left[h r^{\square 1}\right]=0.00653 P_{v a c} \ln \left(1 / \square_{a c c}\right)
$$

Assuming a conservative energy acceptance fraction of $\square_{\mathrm{acc}}=0.001$, the bremsstrahlung lifetime for residual gas pressures from $10^{-4}$ to $10^{-8}$ Torr is $0.8-8,000$ seconds. While admittedly short by storage ring standards, this is well in excess of recirculating linac requirements.

\section{Elastic Scattering}

An electron passing through background gas may also experience an elastic collision (Coulomb scattering) that causes an angular deflection large enough to give the particle a betatron oscillation amplitude larger than the limiting physical or dynamic aperture of the accelerator. For planning purposes, and to be consistent with the collective beam effects discussed above, a limiting physical rectangular aperture of $9 \mathrm{~mm}$ vertical and $40 \mathrm{~mm}$ horizontal has been assumed. In accordance with good machine design practice, we assume that the dynamic aperture will exceed the physical aperture throughout the accelerator. From Turner [2], the limiting aperture can be represented by the setting the admittance, $A_{x}$, where

$$
A_{x}=a_{x}(s)^{2} / \square(s)
$$

$a_{x}(s)$ is the limiting physical aperture and $\square(s)$ is the local betatron function to

$$
A_{x}=\square_{x} \square_{A x}
$$

Since a loss rate for the accelerator as a whole is required, $\square_{x}$ may be taken to be the average betatron function over the arc sections and $\square_{A x}$ is the scattering angle corresponding to the limiting admittance. For the present study, an average betatron function of $30 \mathrm{~m}$ has been selected based on MAD output for the lattice as presently configured plus an additional 50\%. A worst-case local betatron function of $90 \mathrm{~m}$ has been used based on the highest value found anywhere in the lattice for either $x$ or $y$-axis. Similar equations can be formulated for the other transverse dimension. The beam lifetime, $\square_{\mathrm{g}}$, from Turner [3] for a rectangular aperture is; $\square_{b g}=$

$\left[n c\left(\frac{2\left(Z e_{c g s}\right)^{2}}{p c}\right)^{2}\left(\sqrt{\frac{\square_{x} \square y}{A_{x} A_{y}}}+\frac{\square_{y}}{A_{y}} \arctan \left(\sqrt{\frac{A_{x} \square y}{\square_{x} A_{y}}}\right)+\frac{\square x}{A_{x}} \arctan \left(\sqrt{\frac{A_{y} \square_{x}}{\square_{y} A_{x}}}\right)\right)\right]$

Note the dimensions of the quantities used in this equation are in gaussian units. The quantity $Z e_{c g s}$ is the charge of the gas nucleus. As before, a nitrogen equivalent composite residual gas has been assumed. The beam energy term, $p c$ (ergs), is the product of momentum 
and the speed of light. The density of gas molecules, $n$, in molecules per $\mathrm{cm}^{2}$, is directly proportional to the residual gas pressure at a given temperature. The fractional loss of beam for each beam energy level (re-circulation pass) is approximately,

$$
\text { Beam loss fraction }=\mathrm{S} /\left(\square_{\mathrm{bg}} \mathrm{c}\right)
$$

where $S$ is the path length of the beam. A plot of the fractional beam loss for each re-circulation pass over a range of residual gas pressures is shown in Figure 15-2.

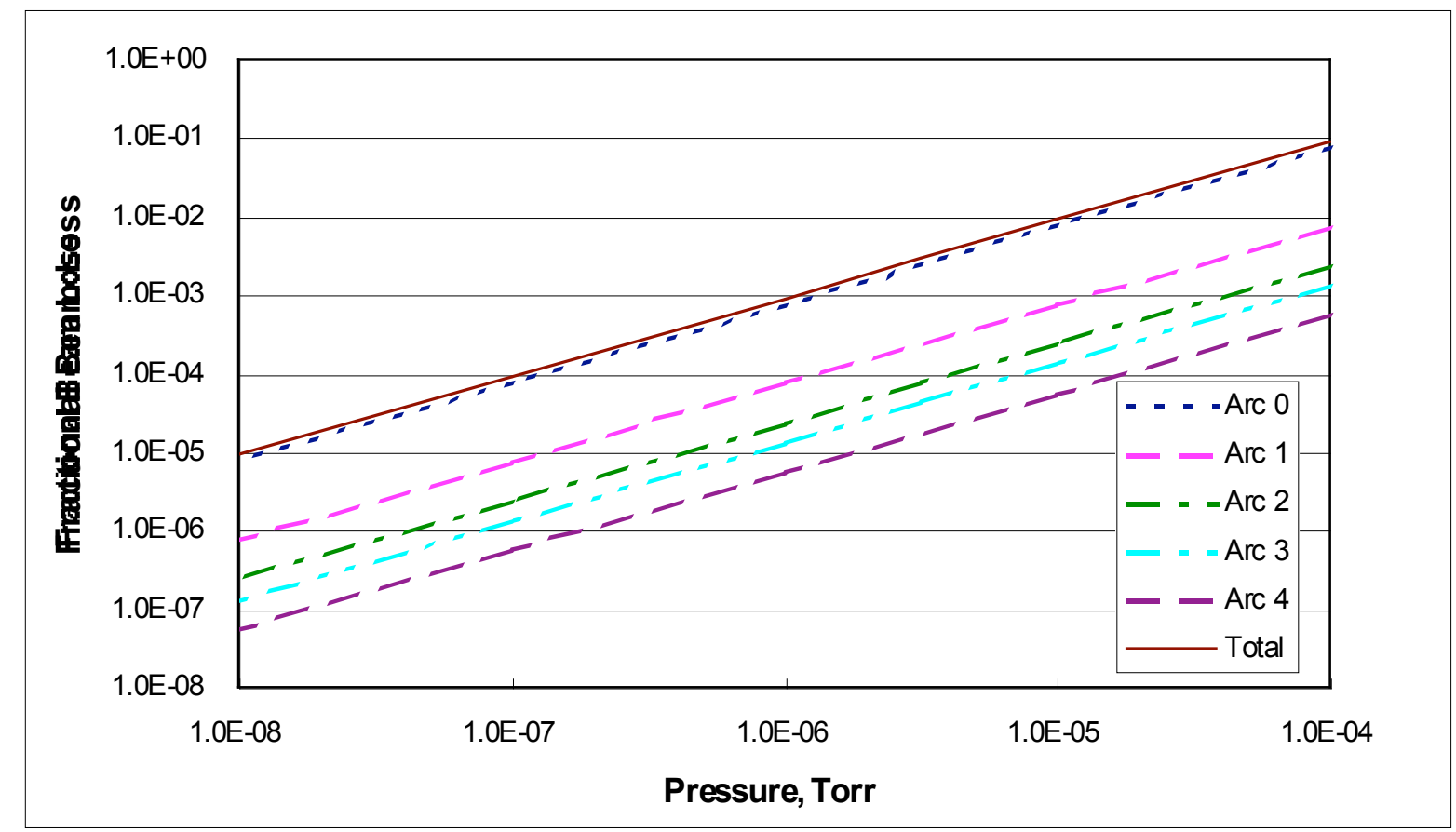

Figure 15-2 Beam loss fraction, due to Coulomb scattering, as a function of residual gas pressure.

The scattered beam will deposit energy on the vacuum chamber wall. This power deposition could lead to elevated wall temperatures and higher outgassing rates. The amount of scattered beam energy is a product of the fractional beam loss, the beam energy and the pulse frequency. On a per meter basis the power deposition, $P_{\text {scat }}$, is

$$
P_{\text {scat }}=b_{\text {rate }} N_{e}\left(\frac{l}{\square b g c}\right) E
$$

where $b_{\text {rate }}=$ bunch rate of $10^{4}$ pulse/sec, and $N_{e}=1.87 \times 10^{10}$, number of electrons in a $3 \mathrm{nC}$ bunch, 
Table 15-1 Line power density on vacuum chamber due to Coulomb scattering (W/m)

\begin{tabular}{|c|r|r|r|r|r|}
\hline Pass $\backslash$ Pressure & $10^{-4}$ Torr & $10^{-5}$ Torr & $10^{-6}$ Torr & $10^{-7}$ Torr & $10^{-8}$ Torr \\
\hline Arc 0 & 3.712 & 0.371 & 0.037 & $3.71 \mathrm{E}-03$ & $3.71 \mathrm{E}-04$ \\
\hline Arc 1 & 0.515 & 0.051 & $5.15 \mathrm{E}-03$ & $5.15 \mathrm{E}-04$ & $5.15 \mathrm{E}-05$ \\
\hline Arc 2 & 0.277 & 0.028 & $2.77 \mathrm{E}-03$ & $2.77 \mathrm{E}-04$ & $2.77 \mathrm{E}-05$ \\
\hline Arc 3 & 0.189 & 0.019 & $1.89 \mathrm{E}-03$ & $1.89 \mathrm{E}-04$ & $1.89 \mathrm{E}-05$ \\
\hline Arc 4 & 0.144 & 0.014 & $1.44 \mathrm{E}-03$ & $1.44 \mathrm{E}-04$ & $1.44 \mathrm{E}-05$ \\
\hline
\end{tabular}

The results of these scattering calculations indicate that inelastic scattering is not a significant effect for the proposed geometry and fractional beam loss from elastic scattering will be less than $0.1 \%$ for residual gas pressures up to $2 \times 10^{-6}$ Torr. As shown in Figure 15-1, most of the beam loss from elastic scattering occurs in the arc between the injector and linac (Arc 0). This suggests that the residual gas pressure limits should be different for the various re-circulation passes. Commensurate with the modest beam losses, the power deposition on the vacuum chamber is relatively insignificant for residual gas pressures as high as $10^{-5}$ Torr.

\section{VACUUM SYSTEM LAYOUT}

The vacuum system must accommodate the magnet lattice as well as the various collective beam effects. With the exception of the first arc and the spreader regions, the lattice consists primarily of identical dipole, quadrupole and sextupole magnets. In cross section these units are identical to the ALS booster ring magnets. Pole gaps in the dipoles are $30 \mathrm{~mm}$ (full height). The radial dimension to the pole tips of the quadrupoles and sextupoles are 32.5 and $35 \mathrm{~mm}$, respectively. These magnets set the maximum external dimensions of the beam tube throughout the accelerator. Collective beam effects dictate the inner height of the beam tube (vacuum chamber) within bend magnets.

The residual gas pressure requirements appear to be very modest. For design purposes a conservative upper limit on the average gas pressure of $1 \times 10^{-6}$ Torr will be used. As with any vacuum system, the pressure is determined by the amount of gas introduced, the conductance of the gas to the pumps and the pump speed. The gas load is a product of thermal outgassing and photon induced desorption.

The amount and mixture of gas liberated from the vacuum wall is a function of the material selected and its preparation. Aluminum is the primary candidate for the vacuum tube because it is relatively inexpensive to extrude, has good vacuum properties, is compatible with commercially available UHV fittings, has adequate electrical conductivity, and has a good track record in other synchrotron light sources. For the purposes of this study, a vacuum baked $\left(150^{\circ} \mathrm{C}\right.$ for $24 \mathrm{hrs}$.) aluminum beam tube and "conflat" type metal sealed fittings are assumed.

The vacuum system presented here consists of an extruded rectangular beam tube in the bend magnet locations transitioning to a circular tube in the straight sections. The external height of the rectangular beam tube is 1 to $2 \mathrm{~mm}$ smaller than the magnet pole tip gap. The internal height 
of the rectangular tube varies with each arc as determined by collective effects described previously; $9 \mathrm{~mm}$ in Arc 0, $12.8 \mathrm{~mm}$ in Arc 1, $16.9 \mathrm{~mm}$ in Arc 2, $20 \mathrm{~mm}$ in Arc 3, $20.9 \mathrm{~mm}$ in Arc 4. The beam tube is connected periodically to a large diameter aluminum pipe that serves as a pumping manifold. A series of modular cryosorption pumps are distributed along the manifold to provide the primary pumping. A small number of turbomolecular pumps backed by oil-free roughing pumps will provide the initial system vacuum. The roughing pumps will also provide pumping for cryopump regeneration, see Figures 15-3, 15-4.

While admittedly a distributed cryopumped system is not typical for storage ring synchrotron light sources, it is widely used in other large accelerators. The advantage of this type of system is that all of the components are readily available commercially. Secondly, the beam tube has a very simple, regular cross section that facilitates close tolerance extrusion. And, third, repair or replacement of the primary pumps can be performed without bringing any portion of the beam tube up to air.

The proposed system contains several variables that can be optimized based on the conditions within a given region of the accelerator. These variables include the width of the beam tube, spacing between manifold-beam tube connections, manifold diameter and cryopump spacing. While each portion of the accelerator section will behave differently, of primary concern are the bend magnets where gas production is the greatest and gas conductance is the least.

\section{Synchrotron radiation induced outgassing.}

The equations derived by Mathewson, et al. [3] are used in the following section to calculate outgassing rates due to synchrotron radiation. Gas is desorbed from the beam tube surface by thermal desorption and by photoelectrons that are generated by incident synchrotron radiation. The energy and quantity of the synchrotron radiation is a function of the photon critical energy. The critical energy, $\square_{c}$, of the photon is a function of the beam energy and the bending radius:

$$
\square_{c}=2.210^{3} \frac{E^{3}}{\square}
$$

where the photon energy $\square$ is in $\mathrm{keV}$, the beam energy $E$ is in $\mathrm{GeV}$ and $\square$ is the bend radius in m. The total photoelectron flux, $\square$, at normal incidence per $\mathrm{mA}$ of beam is approximated by

$$
\square=1.51 \square 10^{14} \frac{\square\left[c^{/ 3}\right.}{E^{2}}\left[2.61 \int_{10}^{1560} \square^{2 / 3} \square^{0.94} d \square+441.9 \int_{1560}^{\square c} \square^{2 / 3} \square^{1.13} d \square\right]
$$

where $E$ is the beam energy in $\mathrm{GeV}$, and $\square$ is the magnetic bend radius in meters. For critical energies below $1560 \mathrm{eV}$ the second integral is not valid and the term is dropped. Photons with energies below $10 \mathrm{eV}$ do not generate photoelectrons in aluminum. The angle of incidence of the photons on the vacuum tube wall will be in the range of 5 to 13 degrees. A worst-case assumption of a $(1 / \sin \square)$ dependence has been used. The number of photoelectrons produced per meter of vacuum chamber, $N_{p e}$, (arc sections only) is obtained by multiplying the flux $\square$ by the 
(1/ sin $\square)$ term and beam current, I ( $0.03 \mathrm{~mA})$, and dividing by the equivalent length of chamber, $L_{\text {arc }}$, receiving the radiation.

$$
\mathrm{N}_{\mathrm{pe}}=\frac{\square \mathrm{I}}{2 \mathrm{~L}_{\text {arc }} \sin (\square)}
$$

The angle of incidence, $\square$, is a function of the beam tube width $w$ and bend radius given by:

$$
\square=\cos ^{-1}\left(\frac{U}{\square+\frac{W}{2}}\right)
$$

The results are summarized in Table 15-2.

Table 15-2 Photoelectron yield per meter for a $6 \mathrm{~cm}$ wide aluminum beam tube.

\begin{tabular}{|c|c|c|c|c|c|c|}
\hline Location & $\mathrm{L}_{,}, \mathrm{eV}$ & $\square$ & $\square, \mathrm{m}$ & $\mathrm{L}_{\text {arc }}, \mathrm{m}$ & $\square, \mathrm{deg}$ & $\mathrm{N}_{\text {pe }}, 1 /(\mathrm{m}-\mathrm{sec})$ \\
\hline Arc 0 & 3 & 0 & 1.20 & 3.6 & 12.68 & 0 \\
\hline Arc 1 & 619 & $3.3 \times 10^{16}$ & 2.30 & 9 & 9.20 & $3.4 \times 10^{14}$ \\
\hline Arc 2 & 2000 & $5.5 \times 10^{16}$ & 4.59 & 18 & 6.53 & $4.0 \times 10^{14}$ \\
\hline Arc 3 & 4176 & $9.1 \times 10^{16}$ & 6.88 & 27 & 5.34 & $5.4 \times 10^{14}$ \\
\hline Arc 4 & 7720 & $11.2 \times 10^{16}$ & 8.49 & 34 & 4.81 & $5.9 \times 10^{14}$ \\
\hline
\end{tabular}

Gas is desorbed from the beam tube surface by the impingement of photoelectrons. For vacuum baked aluminum the desorption efficiency, $\square$ (molecules/electron), is roughly equal to $0.1-0.15[3,4]$. In typical synchrotron light sources this value is reduced by many orders of magnitude through beam scrubbing. However the required circulating currents and durations $(\sim$ $10^{6} \mathrm{~mA}$-hours) are far in excess of re-circulating linac capabilities. The amount of gas desorbed by the beam due to photoelectrons is,

$$
\mathrm{Q}_{\mathrm{pe}}[(\text { Torr- } \mathrm{l} / \mathrm{s}) / \mathrm{m}]=2 \mathrm{~L} \mathrm{~K}
$$

where $\mathrm{K}^{-1}$ is equal to the number of molecules per Torr-liter of gas at room temperature $(\mathrm{K}=$ $\left.3.03 \times 10^{-20}\right)$. The factor of 2 is included because gas molecules are desorbed as the photoelectron leaves one surface and arrives at another. The efficiency of gas evolution by electron bombardment of aluminum peaks at electron energies of about $400 \mathrm{eV}$ and then falls asymptotically to a relatively constant value above $2 \mathrm{keV}$ [4]. Rough average values for $\square$ over the $0.1-7 \mathrm{keV}$ range were selected, as shown in Table 15-3. Calculated gas desorption rates, using these species specific $\square$ values, are also shown in this table. 
The power deposition on the vacuum tube wall from synchrotron radiation is a possible source of heating and therefore enhanced thermal desorption. The amount of synchrotron power, estimated from emission of an electron beam in uniform circular motion [5], results in a peak linear power density, $5.4 \times 10^{-3} \mathrm{~W} / \mathrm{cm}$. Under worst-case assumptions [6], this power is deposited over a width of $0.24 \mathrm{~mm}$ resulting in a peak heat flux of only $0.5 \mathrm{~W} / \mathrm{cm}^{2}$. This level of localized heating is negligible for a reasonably thick aluminum beam tube and will not result in increased thermal outgassing.

Table 15-3 Photoelectron desorption efficiency and gas desorption rates per meter of beam tube by gas species.

\begin{tabular}{|l|l|l|l|l|l|r|}
\hline \multicolumn{1}{|c|}{ Gas } & $\mathrm{h}$ & $\operatorname{Arc} 0$ & $\operatorname{Arc} 1$ & $\operatorname{Arc} 2$ & $\operatorname{Arc} 3$ & Arc 4 \\
\hline Hydrogen & 0.1 & - & $1.9 \times 10^{-6}$ & $2.3 \times 10^{-6}$ & $3.1 \times 10^{-6}$ & $3.3 \times 10^{-6}$ \\
\hline CO & 0.03 & - & $5.8 \times 10^{-7}$ & $6.8 \times 10^{-7}$ & $9.2 \times 10^{-7}$ & $1.0 \times 10^{-6}$ \\
\hline CO2 & 0.025 & - & $4.8 \times 10^{-7}$ & $5.7 \times 10^{-7}$ & $7.6 \times 10^{-7}$ & $8.3 \times 10^{-7}$ \\
\hline Methane & 0.008 & - & $1.5 \times 10^{-7}$ & $1.8 \times 10^{-7}$ & $2.4 \times 10^{-7}$ & $2.7 \times 10^{-7}$ \\
\hline Total & & & $3.1 \times 10^{-6}$ & $3.7 \times 10^{-6}$ & $5.0 \times 10^{-6}$ & $5.4 \times 10^{-6}$ \\
\hline
\end{tabular}

\section{Thermally induced outgassing.}

The quantity and composition of thermally desorbed gas is substantially altered by a moderate vacuum bake-out in aluminum systems, as shown in Table 15-4. Water is the dominant species in unbaked aluminum whereas hydrogen is most prolific in baked aluminum systems.

Table 15-4 Outgassing rates measured for an aluminum vacuum chamber after $100 \mathrm{hrs}$ of pumping from [4] as shown in [2].

\begin{tabular}{|l|c|c|}
\hline \multirow{2}{*}{ Gas } & \multicolumn{2}{|c|}{ Q (Torr-liters $/$ sec-cm $^{2}$ ) } \\
\cline { 2 - 3 } & Unbaked & Baked $^{*}$ \\
\hline Hydrogen & $7 \times 10^{-12}$ & $5 \times 10^{-13}$ \\
\hline Methane & $5 \times 10^{-13}$ & $5 \times 10^{-15}$ \\
\hline Water & $3 \times 10^{-10}$ & $<1 \times 10^{-15}$ \\
\hline Carbon monoxide & $5 \times 10^{-12}$ & $1 \times 10^{-14}$ \\
\hline Carbon dioxide & $5 \times 10^{-13}$ & $1 \times 10^{-14}$ \\
\hline \multicolumn{2}{|c|}{${ }^{*} 150^{\circ} \mathrm{C}$ for 24 hours } \\
\hline
\end{tabular}

All metal surfaces, including the beam tube, manifold and connecting tubulation are assumed to be clean, baked aluminum. The amount of thermal outgassing is the product of the rates shown in Table 15-4 and the surface area. For the beam tube of Arc $4(2.1 \mathrm{~cm} \times 6 \mathrm{~cm})$ the gas produced per meter is $8.5 \times 10^{-10}$ Torr-liter/sec or 6,000 times smaller than synchrotron radiation induced desorption. 


\section{Gas Pressure Profile}

The most difficult pumping conditions are present in the arc sections due to the limited gas conductance and the synchrotron radiation induced gas load. The pumping of the straight sections is relatively straightforward in comparison and is not considered in this study. Given the assumption of a discretely pumped system, a rational configuration would include widely spaced pumps, a manifold sized to be consistent with the pump size and a limited number of beam tube penetrations. A system that meets the criteria consists of CTI- 8 cryopumps centrally located on a 10 meter span of $20 \mathrm{~cm}$ diameter manifold. Short, $30 \mathrm{~cm}$ long by $5 \mathrm{~cm}$ diameter tube and bellows assemblies connect the manifold to the beam tube. An illustration of the vacuum system concept, as applied to Arc 4, is shown in Figure 15-3. A representative section of the system is shown schematically in Figure 15-4. Note that a single cryopump services a segment of Arc 4 containing 9 dipole magnets.

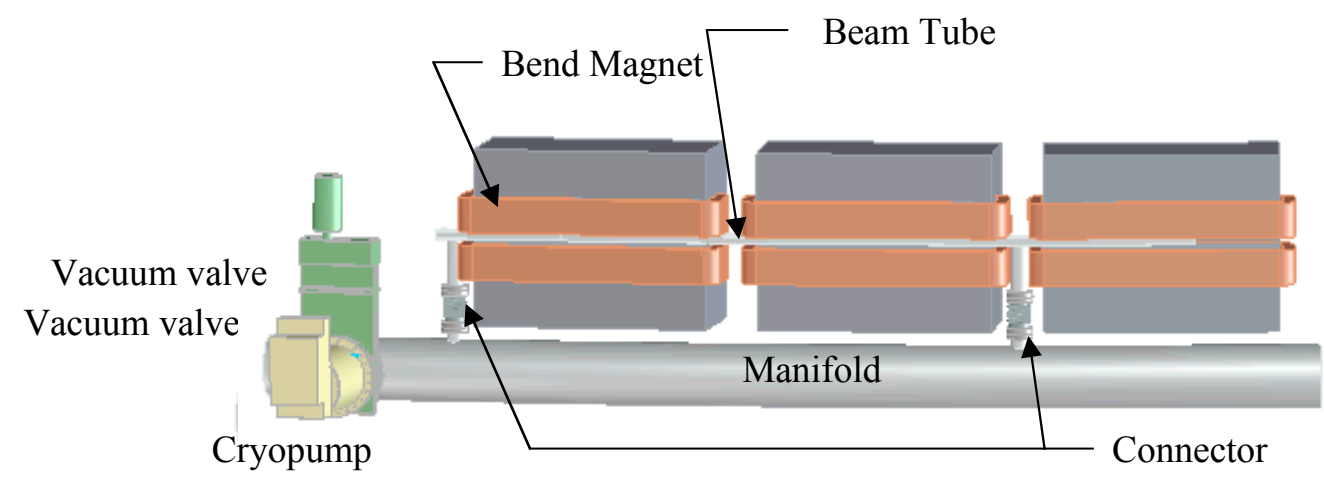

Cry

Floor

Figure 15-3 Conceptual layout of a representative section of the vacuum system.

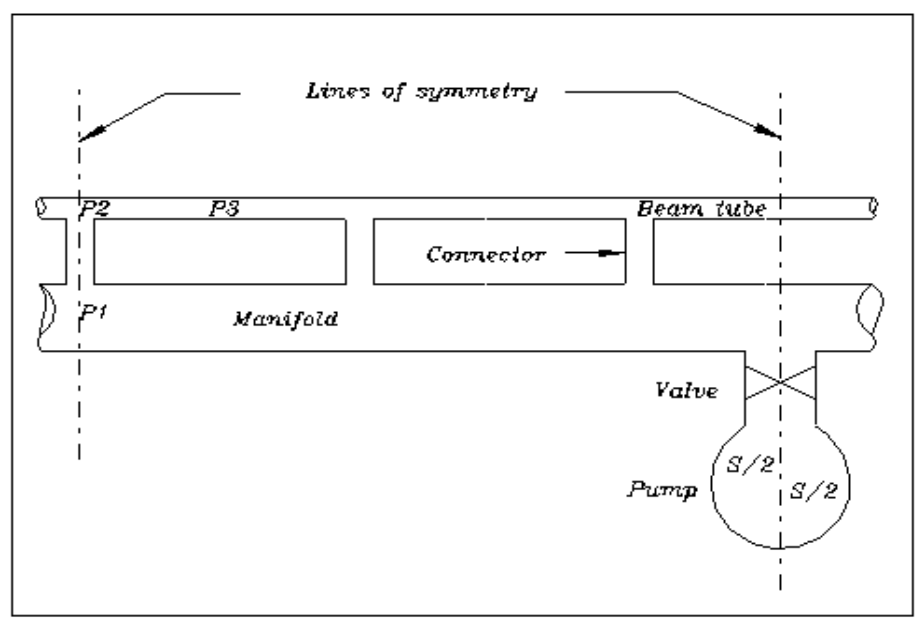

Figure 15-4 Schematic of a typical vacuum system section. 
In addition to the outgassing rate, the pressure in the beam tube is a function of the gas conductance and pump speed. For ease of computation, gas evolved from the beam tube, connector tubing and manifold is treated as a single continuous distributed source along the length of the manifold. Using this assumption, the peak pressure in the manifold, P1, is given by:

$$
\mathrm{P} 1=Q L\left(\frac{1}{2 C_{L}} \frac{1}{S_{p}}\right)+P_{\text {base }}
$$

where $Q$ is the outgassing rate in Torr-liters/sec per meter of beam tube, $C_{L}$ is the conductance of the manifold (from P1 to pump) in liters/sec, $S_{p}$ is the pumping speed (half of the full pump speed) in liters/sec, $L$ is half of the span between pumps in meters, and $P_{\text {base }}$ is the base pressure of the pump in Torr.

The conductance of the cylindrical tubing was calculated using Santeler's [7] correction to the common formula for a circular tube in molecular flow. Assuming the tube terminates into a larger volume, the manifold's conductance is from 1000 to 5000 liters/sec, depending on the gas species.

The pressure at the connecting tube entrance, $\mathrm{P} 2$, is determined by adding the pressure differential from gas flow through the connecting tube to the maximum pressure in the manifold, $\mathrm{P} 1$. The peak pressure in the beam tube, $\mathrm{P} 3$, is found by adding the maximum pressure increase along the beam tube, between connectors, to the pressure at the connector entrance, P2.

The conductance of the beam tube, $C_{\text {tube }}[$ [iter/s], is calculated using the equation from Roth [8] for a long rectangular tube of uniform cross section,

$$
C_{\text {tube }}=\frac{8}{3} \sqrt{\frac{k T_{g}}{2 \square m}} 1000\left(\frac{w^{2} b^{2}}{(w+b) L_{r}} K_{c}\right)
$$

where $k$ is the Boltzmann constant, $1.385 \times 10^{-23} \mathrm{~J} / \mathrm{K}$,

$T_{g}$ is the gas temperature in $\mathrm{K}$,

$m$ is the mass of the molecule in $\mathrm{kg}$,

$L_{r}$ is the half-span between connectors $\left(\mathrm{L}_{\mathrm{r}}=\mathrm{L}_{\text {tube }}\right)$

$b$ is the full internal height of the beam tube,

$w$ is the internal width of the beam tube, and

$K_{c}$ is a constant that is a function of b/w (1.2 for present case).

The partial pressures in Arc 4 at P1 - P3 for the four dominant gas species are shown in Table 15-5. The base pressure of the cryopump is assumed to be $10^{-9}$ Torr, divided evenly between gas species. Actual base pressure of a properly maintained pump should be lower. All other arcs produce lower background pressure because the lower synchrotron radiation induced outgassing more than compensates for the reduced gas conductance. 
Table 15-5 Partial pressures at key points (see Fig. 15-4) in the vacuum system.

\begin{tabular}{|l|c|r|r|r|}
\hline & $\mathrm{Sp}(=\mathrm{S} / 2), 1 / \mathrm{s}$ & \multicolumn{1}{c|}{ P1, Torr } & \multicolumn{1}{c|}{ P2, Torr } & \multicolumn{1}{c|}{ P3, Torr } \\
\hline Hydrogen & 1250 & $2.7 \times 10^{-8}$ & $7.5 \times 10^{-8}$ & $1.26 \times 10^{-7}$ \\
\hline $\mathrm{CO}$ & 750 & $2.2 \times 10^{-8}$ & $7.5 \times 10^{-8}$ & $1.32 \times 10^{-7}$ \\
\hline $\mathrm{CO}_{2}$ & 1250 & $1.9 \times 10^{-8}$ & $7.5 \times 10^{-8}$ & $1.34 \times 10^{-7}$ \\
\hline Methane & 950 & $0.5 \times 10^{-8}$ & $1.6 \times 10^{-8}$ & $0.28 \times 10^{-7}$ \\
\hline \multicolumn{1}{|c|}{ Total } & - & $7.3 \times 10^{-8}$ & $2.4 \times 10^{-8}$ & $4.20 \times 10^{-7}$ \\
\hline
\end{tabular}

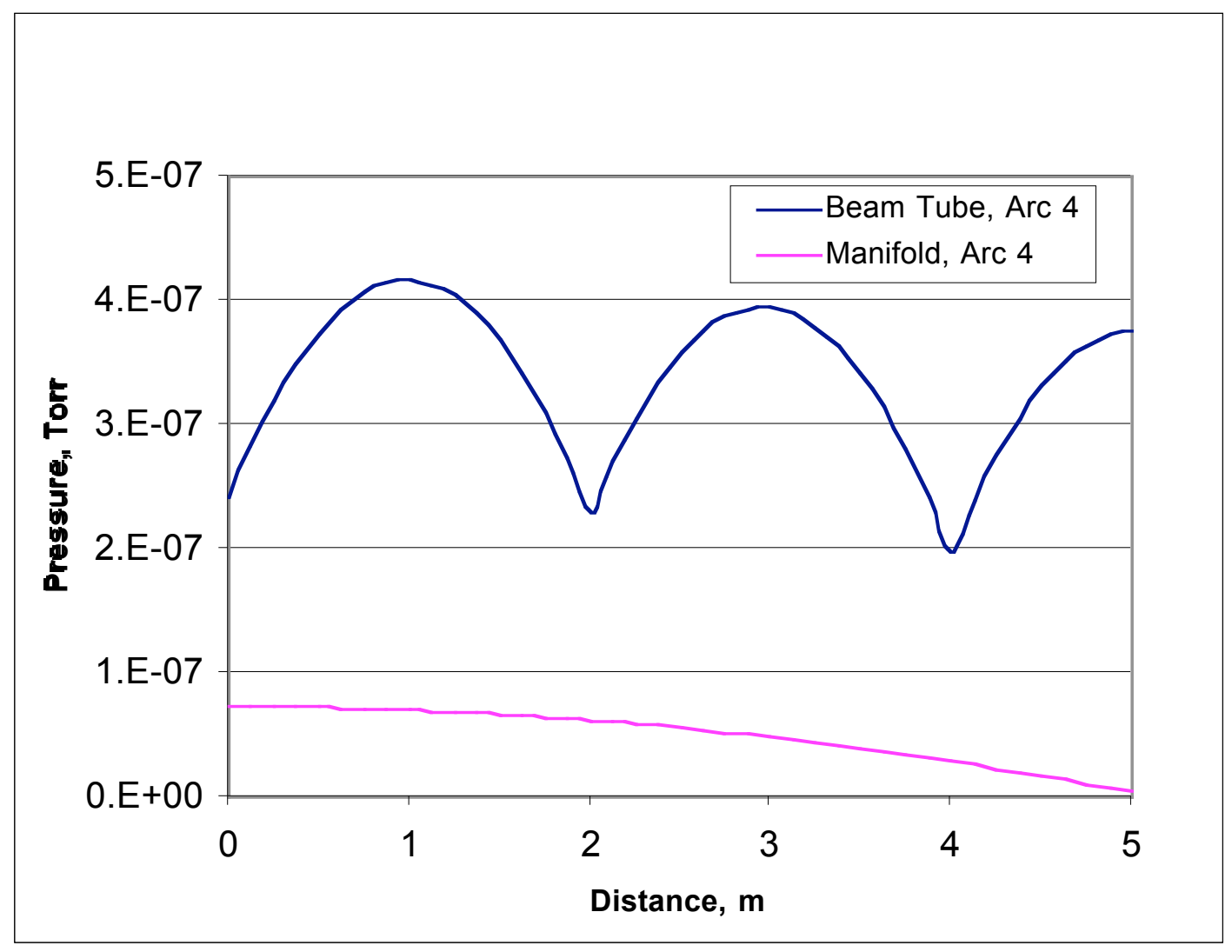

Figure 15-5 Pressure profile along a representative portion of the Arc 4 beam tube

The plot shown in Figure 15-5 is an approximate pressure profile of the Arc 4 beam tube derived by applying the methodology used to find pressures P1-P3 to the other analogous positions along the beam tube and then applying a parabolic profile between them. By inspection, the average pressure along the path of the beam is $3 \times 10^{-7}$ Torr. This average residual gas pressure compares favorably with the $1 \times 10^{-6}$ Torr design limit. 


\section{REFERENCES}

[1] H. Wiedemann, Particle Accelerator Physics I-Basic Principles and Linear Beam Dynamics, Springer-Verlag, Berlin, pp.378-380, 1999

[2] W. C. Turner, Notes for a Course on Accelerator Vacuum Physics, CBP Tech. Note 188, June 11, 1999.

[3] A. G. Mathewson, G.H. Horikoshi, and H. Mizuno, Some Notes on the Photoelectron Induced Gas Desorption Problems in the Photon Factor and Tristan, National Lab for High Energy Physics Report, Japan.

[4] M. Achard, Electron and Ion Induced Gas Desorption from Stainless Steel, OFHC Copper, Titanium and Pure Aluminum, CERN-ISR-VA/76-34

[5] M. Sands 1970 (from PEP-II Conceptual Design Report)

[6] PEP-II Conceptual Design Report, June 1993, p. 305.

[7] D. J. Santeler, New Concepts in Molecular Gas Flow, J.Vac. Sci. Technol. A, Vol. 4, No. 3, May/June 1986

[8] Roth, Vacuum Technology $2^{\text {nd }}$ Ed., North-Holland, 1982 pp. 84-87. 


\section{BEAM DIAGNOSTICS}

\section{INTRODUCTION}

The implementation of the beam diagnostics philosophy for the proposed ultrafast x-ray source [1] will proceed in two successive phases. In the initial phase efforts will focus on meeting the requirements necessary to characterize and commission the various sections of the machine. This will require the complete assessment of the beam properties. The operations phase of the instrumentation will then require the implementation of diagnostics targeted to ensure a fully reliable facility for users. In this chapter we review instrumentation and detection techniques suitable for these diagnostics and briefly describe recent examples of their use. We also consider technical approaches that can contain the cost of diagnostics without compromising machine tunability or optimum performance.

\section{DIAGNOSTIC REQUIREMENTS}

The four-pass acceleration scheme drives the diagnostics philosophy. Beam properties will always be measured with individual bunches and beam quality will not be affected by resonant or cumulative effects that are present in storage rings. Specific procedures and techniques will be developed to measure the electron optical parameters of the accelerator at a $10 \mathrm{KHz}$ repetition rate. Data acquisition and analysis must be performed accordingly. Procedures developed and practiced at single-pass accelerator facilities, such as the SLAC SLC and the TJNAF CEBAF can be adopted and improved to meet diagnostics requirements. The instrumentation accuracy performance will be focused on the specifics of different sections of the accelerator. For example, the resolution of the same type of monitors, such as beam position or beam loss monitors, could be less stringent in some sections of the machine thus making their implementation more cost effective. Different sections of the accelerator will require that various specific beam properties be controlled differently.

The impedance budget will need to be carefully evaluated [2] as the very high longitudinal bunch charge density will create strong wake fields in the linac structures and the accelerator vacuum chamber [3]. This effect, in conjunction with rf non-linearities and higher order mode dispersion, will generate banana-type bunch configurations that will need to be detected and damped for proper operation.

Specifications for the sensitivity of beam loss and beam position monitors will need to be more stringent in the spreader, sections where beam loss rates may be higher, than elsewhere. A more detailed discussion may be found in Chapter 4-Collective Effects.

Amplitude and phase tuning of the dipole rf cavities, to achieve the optimal crabbing angle for hard x-ray production, must be implemented with feed-back with a proper bunch-tilt diagnostic.

At the base of sub-picosecond hard x-ray compression is the production of a >50:1 horizontal to vertical emittance aspect ratio and its conservation, throughout the acceleration stages of the machine. Consequently, emittance monitoring and control must be given special attention. 
A highly sensitive transverse beam position monitoring system coupled with a feedback/feedforward system is mandatory in the photon production section to ensure the necessary transverse beam stability for effective operation of the hard x-ray beamlines.

\section{ObServables ANd Beam Properties}

During both the commissioning and the operational phases, the control of the accelerator will rely upon beam characterization based on following beam observables:

- Transverse beam position,

- Transverse beam profiles,

- Bunch charge,

- Longitudinal charge distribution, and

- Transverse-longitudinal correlation among bunch particles.

Primary beam structural information is derived from both observed optical properties and electrical structural characteristics. These observables provide the main ingredients for understanding the accelerator and we must build tools to reliably control its operation through their quantification.

The characterization and tuning of the accelerator is based essentially upon the following beam properties:

- Closed orbit and/or beam-line trajectory,

- Beam emittance,

- Betatron functions at specific sections (as determined by beam monitors, deflecting cavities, light extraction line, etc),

- Dispersion function (both design and error induced),

- Betatron coupling,

- Betatron phase advance beta beating and

- Beam energy spread.

\section{BEAM PARAMETERS AND INSTRUMENTATION}

Diagnostic techniques and related instrumentation approaches of interest for the facility are reviewed in the following sections and examples are given of recent applications in existing facilities. More detailed explanations and references are collected in ref. [4].

\section{Radiation and Beam Diagnostics}

The physical characteristics of radiation emitted by charged particles during their interaction with the environment can be exploited to record footprints useful to the determination of beam properties. Coherent Radiation (CR) from a bunch of $N$ charged particles produces much higher power than that of incoherent radiation as it is proportional to the square of the bunch population [5]

$$
P(\square)=N \quad p(\square)[1+N \quad f(\square)]
$$


where $p(\square)$ is the power radiated by a single electron at the frequency $\square$.

The term $N^{2} p(\square) f(\square)$ is the coherent contribution which the phase relation between electrons in the bunch. The bunch form factor, $f(\square)$, is related to the electron distribution function. The CR spectrum carries information on $f(\square)$ and therefore contains information on the bunch-length.

\section{Intercepting techniques}

Intercepting techniques make use of radiation emitted by a relativistic charged particles in a medium. A relativistic particle emits Cherenkov Radiation (ChR) when travelling in a medium with a velocity $\mathbf{v}$ larger than the phase velocity $\mathbf{v}_{\mathbf{p}}$ at the frequency $\square$ in the medium [6]:

$$
\square^{2} \square(\square)>1 \text { or } v>\sqrt{\square} \frac{c}{n(\square)}=v_{p}(\square) \sqrt{\square}
$$

Cherenkov Radiation is emitted in a cone of semi-aperture $\square_{c}=\cos ^{\square 1}[\sqrt{\square} / \square n(\square)]$. As the refraction index $n(\square)$ varies with the frequency of the emitted radiation, a particle radiates ChR under different angles. This property is usually exploited in high energy physics detectors for velocity measurements using narrow band filtering and particle selection via the control of the index of refraction of the gas.

A particle crossing the interface between media with different dielectric constants emits Transition Radiation (TR) [7]. For normal incidence the angular distribution shows a typical twolobe pattern, confined within a cone with of an 1/】 opening angle centered about the particle velocity direction.

Optical Transition Radiation (OTR) monitors [8] are widely used to measure transverse profiles. Backward and forward OTR is emitted at both sides of a thin screen inserted at $45^{\circ}$ in the beam. A large amount of information about the beam position, spatial distribution, intensity, emittance and energy may be derived from both directons [9]. Although forward OTR is more intense, the use of the backward OTR is more practical in terms of light extraction.

\section{Non-intercepting Techniques}

Synchrotron Radiation (SR), Diffraction Radiation (DR) and the linear electro-optic (Pockels) effects are the main sources for non-intercepting diagnostics.

Visible synchrotron radiation is generated in dipoles or in dedicated periodic magnetic structures (wigglers, undulators) whose characteristics in terms of period $\square_{u}$ and magnetic field $B_{o}$ are determined, for the given beam energy, by the wavelength $\square_{\mathrm{n}}$ to be detected:

$$
\square_{n}(\square)=\frac{\square_{u}}{2 n \square^{2}}\left(1+\frac{K^{2}}{2}+\square^{2} \square^{2}\right), \quad K=\frac{e B_{o} \square_{u}}{2 \square m c}=\square
$$

where $K$ is the deflection parameter and $\square=K / \square$ the maximum angular deflection of the beam within the wiggler/ undulator.

In undulators $(K<1, \square \square 1 / \square$ radiation from successive periods interferes coherently as the angular beam excursion is within the nominal $1 / \square$ radiation cone. This produces sharp peaks at 
harmonics $n$ of the fundamental $\square_{1}$ and dedicated detectors may be chosen for optimum response at selected peaks.

A relativistic charged particle generates DR when traversing an aperture (slit or hole) in a conducting screen [10]. The DR intensity is characterized by a cut-off frequency $\square_{\mathrm{c}}=\mathrm{c} \square / a$ where $a$ is the slit aperture or the hole diameter. DR intensity is generally lower than TR, but the non-intercepting properties of the detector make the technique of particular interest for bunch length measurements.

The Pockels effect [11] exploits the birefringence induced on an electro-optic crystal by an external electric field. As the field produced by a relativistic charged bunch propagates perpendicularly to the direction of motion it can induce birefringence in an electro-optic crystal located in the vicinity of the bunch trajectory. The analysis of phenomena connected with the induced birefringence, when proper temporal resolution requirements are satisfied, can provide a measurement of bunch lengths within the ps range.

\section{Transverse EmitTANCE}

In contrast to a storage ring, the four pass recirculating linac facility is less sensitive to vertical emittance growth from betatron coupling and error induced vertical dispersion. Nevertheless, preserving the very low emittance and its high aspect ratio are a key requirements in the performance of the facility and efficient emittance diagnostics at different sections of the beam path are mandatory. The rms transverse beam profile of a gaussian beam at a section $s$ of a beam line is the composition of the betatron and the synchrotron rms distributions:

$$
\square^{2}(s, E)=\square(E) \square(s)+\left[\square(s) \square_{e}(E)\right]^{2}
$$

where $\square(s)$ and $\square(s)$ are the local betatron and dispersion functions, $\square_{\mathrm{e}}(E)$ and $\square(E)$ the energydependent momentum dispersion and transverse emittance.

Combining profile measurements at two locations, one of which dispersion-free, (for example at the exit of the injection rf gun), both the emittance $\square$ and the energy spread $\square_{e}$ can be unfolded from equation (4). In order to arrive at this information. The $\square$ - or $\square$-functions at the radiation emission points or at the profile measurement section must be known, depending upon whether the detection is accomplished through intercepting or non-intercepting.

An alternative method consists in taking successive profile measurements at the same location by varying the focusing strength of a quadrupole of which the transfer matrix is known.

\section{Synchrotron Radiation Monitors (SRM)}

Synchrotron radiation in the visible range, is generated in dipoles or in dedicated periodic magnetic structures (wigglers, undulators), SRMs use this radiation to image transverse beam distributions onto (intensified) charge coupled devices (ccd) for digital recording and analysis. Short superconducting undulators are planned for use at the LHC [12] to produce visible light for transverse and longitudinal beam diagnostics. 


\section{TR and DR screens}

OTR screens have been used to characterize the low beam emittance at the Argonne APS injector linac [13]. The $\sim 5 \square \mathrm{mm}$-mrad $\mathrm{rf}$ gun emittance was measured via beam profiles obtained from an OTR screen alternated with a $0.5 \mathrm{~mm}$ thick Ce-doped YAG crystal.

In comparing the OTR and Ce-doped YAG single-crystal screens, the OTR screen performed better than the YAG:Ce crystal over the spanned macropulse charge (1 to $7 \mathrm{nC}$ ), in terms of spatial resolution, time response and conversion efficiency.

A non-invasive real-time beam diagnostic has been achieved by adopting fast data conversion from frequency to time domain in an experiment using Coherent Diffraction Radiation (CDR) [14]. Experiments were performed with a $150 \mathrm{MeV}$ beam having a $2.5 \square \mathrm{s}$ macropulse duration and about $0.85 \mathrm{pC}$ single bunch charge. In agreement with Ref. [5] the intensity of the radiation was found to be proportional to the square of the bunch population for either type of screen (hole or slit).

\section{Wire Scanner Monitors (WSM)}

The calibration of optical techniques is usually accomplished by wire scanners, either quartz or beryllium wire a few microns thick travels across the beam produces bremsstrahlung radiation which is observed in order, to reproduce the transverse beam distribution.

Wire arrangements and motion controls have evolved in the last decade to add supplementary information to the standard transverse beam size profile description. Skewed $(x, y)$ distributions, originating from betatron coupling terms along the beam trajectories, have been measured and successfully disentangled at the PEP-II interaction point.

\section{Direct X-Ray Monitors}

A non-intercepting profile monitor exploits the x-ray component of the synchrotron radiation spectrum for improved diffraction resolution. The radiation is emitted either by a wiggler/ undulator magnet or by one of the dipoles in the main ring structure.

\section{Quadrupole Electrical Pickup (PU)}

A completely different approach to non-disrupting methods for emittance measurements has been recently suggested at CERN [15]. A revised analytical treatment of the original idea [16] has confirmed that the BPM response is not only sensitive to the transverse position of the bunch electric center-of-gravity but also to its rms horizontal and vertical sizes at the PU location.

A quadrupole PU has been designed [17] and a prototype is presently being tested at the CERN PS. The performance of this novel device has yet be published but if the anticipated response is confirmed it will represents a simple and cost effective approach to the emittance measurement problem. 


\section{LONGITUDINAL DISTRIBUTIONS}

\section{TR and DR methods}

Incoherent OTR and coherent transition radiation (CTR) have been used to measure micropulse bunch length [18]. OTR screens have shown a fast enough response time $(<100 \mathrm{fs})$ to be used for intercepting measurements in femtosecond time domain. Further developments on bunch length monitoring using CTR autocorrelation are reported in [19].

Recent measurements of sub-picosecond electron beam structure using a non-intercepting technique based on CDR autocorrelation are reported in [20]. In this experiment a thermionic rf gun with $40 \mathrm{MeV}$ has macropulses sent through a $5-\mathrm{mm}$ tall slit in a conducting screen held at $45^{\circ}$ to the beam direction.

\section{Streak Camera (SC)}

Dual-sweep streak cameras are among the most powerful tools used for the observation and monitoring of fast temporal phenomena in the visible range as well as in the (soft) $\mathrm{x}$-ray domain, with temporal resolution in the ps region.

The relatively low sensitivity of the x-ray SC photo-cathodes can be improved by replacing the Au cathode, mainly used for its material stability, with a CsI one [21]. An Au photo-cathode dual-sweep SC operated the APS has been tested on a $248 \mathrm{~nm}(5 \mathrm{eV})$ showing a resolution of the order of $1 \mathrm{ps}$ [22].

A collection of experimental methods used for the measurement of picosecond bunch lengths at energies between 6 and $170 \mathrm{MeV}$ at the TESLA Test Facility (TTF) is reported in [23].

\section{Electro-Optic (Pockels) effect}

Electro-optic sampling techniques based on the Pockels effect [11] have recently been adopted to measure the time duration of electron bunches with sub-picosecond time resolution $[24,25]$.

The birefringence induced in an electro-optical crystal by the electric field associated with a relativistic electron bunch travelling nearby has been detected by monitoring the induced rotation of polarization in an initially linearly polarized optical probe using suitable polarization optics. The bunch duration is then obtained by scanning the delay between the optical probe and the bunch.

The electro-optic Pockels effect can be used to detect temporal properties or other fast propagating fields, such as the wake-fields inside the vacuum chamber.

\section{Radiation Spectrum Fluctuations}

It has been proposed [26] to retrieve the longitudinal properties of an electron bunch from the analysis of fluctuations in the undulator radiation related to noise fluctuations in its longitudinal density. The first experimental results have been obtained on a 1-5 ps, $44 \mathrm{MeV}$ electron bunch [27]. A review paper [28] reports results in the $30 \mathrm{MeV}$ to $30 \mathrm{GeV}$ energy range. The single-shot length of 4 ps long bunch at the APS Low Energy Undulator Test Line (LEUTL) has been analyzed with this technique and experimental results are reported in [29]. 


\section{Closed Orbit / Beam Trajectory}

Four-button electromagnetic Beam Position Monitors (BPM) will detect the transverse position of bunches at given locations in the machine. As the difference signal from opposite electrodes is proportional to the transverse bunch displacement $\square z$ and the bunch population $N_{\mathrm{b}}$, the $\mathrm{s} / \mathrm{n}$ ratio in the BPM electronics must cope with a single pass displacement resolution

$$
e N_{b} \square z=10 \square m n C,
$$

i.e. $10 \square \mathrm{m}$ at $1 \mathrm{nC}$ and $\sim 1 \mathrm{~mm}$ at $0.01 \mathrm{nC}$ bunch charge. The associated electronics should operate in a 0.01 to $5 \mathrm{nC}$ dynamic range.

Although BPMs will be needed throughout the machine, requirements in terms of single pass resolution vary depending on their specific location. However, an efficient and conservative approach keeps the same design for all electronics. The number of detectors and electronic channels can be nonetheless be optimized by a trade between the amount and the quality of the information provided and careful selection of the location of the BPMs. To maximize the sensitivity to orbit kicks from randomly distributed machine misalignments, BPMs are installed, whenever possible, at high- $\square$ locations, i.e. close to quadrupoles. Installing BPMs only at the defocusing quadrupoles does not compromise the orbit quality in the arcs, where accurate vertical plane information is preferred. In the straight sections, one option is to install only one BPM between each QF-QD doublet, where the $\square$-functions have similar amplitudes. This provides the same sensitivity in both planes while halving the number of BPMs.

Given the complete $(x, y)$ symmetry of the orbit in the rf accelerating sections, BPMs cannot be chosen with a (horizontal or vertical preferred) plane. However, in this region intensity information is not required. The same conclusion, though for different reasons, can be drawn for the BPMs in the photon production sections. Here a maximum amount of information is needed to implement on-line trajectory measurements and dispersion-free steering algorithms. Intensity information from BPMs in this region can be reduced to a minimum - that required for the Personal Protection System (PPS) and Machine Protection System (MPS).

A BPM system design has been designed and analyzed. The circuit block-diagram is shown in Figure 16-1. A heterodyne technique uses electrostatic button pickup signals with detection at $3.9 \mathrm{GHz}$ for maximum sensitivity. Individual baseband pickup signals are digitized in a 12-bit $\mathrm{ADC}$, on a turn-by-turn basis (approximately $1 \mathrm{MHz}$ in the main linac). Signal processing then occurs at the $10 \mathrm{kHz}$ bunch rate. Special attention must be paid to the timing system since each turn is of different length, and the analog to digital converters (ADC) for each BPM must be clocked appropriately. By requiring the position signal to be three times the system thermal noise, we find the resolution of the system shown in Table 16-1 for typical component parameters. 


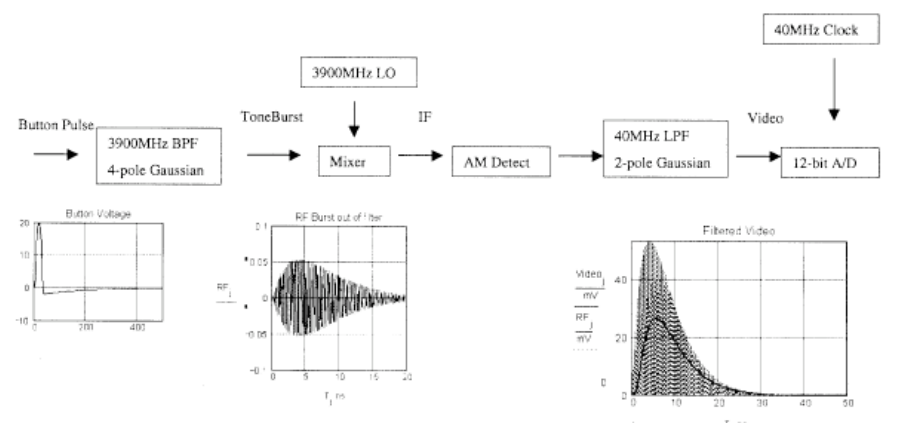

Figure 16-1 BPM electronics block diagram.

Table 16-1 BPM resolution for various bunch charges.

\begin{tabular}{|c|c|}
\hline Bunch charge $(\mathrm{nC})$ & BPM resolution $(\mu \mathrm{m})$ \\
\hline 1 & 7 \\
0.1 & 70 \\
0.01 & 700 \\
\hline
\end{tabular}

\section{Beam Based Alignment}

The large horizontal to vertical emittance aspect ratio forms the basis of sub-picosecond hard $\mathrm{x}$-ray production and the control of factors which can contribute to a vertical emittance growth is critical.

Misaligned quadrupoles and sextupoles are at the root of vertical emittance deterioration through betatron coupling (rotation about the beam axis) as well as error-induced vertical dispersion (off-axis trajectories).

The application of Beam Based Alignment (BBA) techniques in single pass beam lines is complementary to orbit error correction schemes adopted in circular accelerators. The achievable accuracy is higher (a few tens of $\square \mathrm{m}$ ) and each single element may be treated individually making misaligned elements unequivocally identified (which is not always the case when dealing with orbit discontinuities).

Angular deviations to the beam trajectories induced by off-axis passages in a misaligned quadrupole are detected by recording orbit differences as a function of its strength. The misalignment is characterized in amplitude and sign via the $R_{12}$ and $R_{34}$ matrix elements of the beam line segment.

The BBA algorithms implemented for the alignment of the quadrupoles and sextupoles of the SLC transport line $[30,31]$ provides a useful reference. 


\section{Beam Intensity}

Beam Current Transformers (BCT) are generally used for measuring both the average beam current and the peak bunch intensity. In contrast to a storage ring, the complexity of this machine dictates a more distributed measuring scheme. Access to information on the beam intensity distribution along the different beamlines may also be required by the Personnel (PPS) and Machine Protection Systems (MPS), but it can be operationally difficult if a single set of diagnostics serves for both beam characterization and PPS/MPS .

One way of providing a distributed bunch intensity information consists in retrieving the Sum $(\square)$ information from the four buttons of each BPM. This is thus proportional to the total bunch charge. This can be accomplished by retrieving the $\square$ information from the BPM electronics in addition to its normalization role for the up-down and right-left differences needed for position measurement. To reduce the number of transmission channels, the intensity information can be transmitted in multiplex mode together with either a horizontal or vertical position at the same time.

\section{Beam Loss Monitors}

A relatively large number of Beam Loss Monitors (BLM) will be needed to help in the steering of the beams in the spreader sections, where losses can be expected to be important. They will also be necessary and to cope with PPS and MPS requirements.

The anticipated sensitivity $(\sim 0.01 \mathrm{nC})$ is much lower than that required for the LHC project where 3000 mini ionization chambers provide a loss-dependent current signal with a $\sim 10^{7}$ dynamic range.

\section{Synchronization and Digital Signal Processing Optical Feedback and Synchronization}

Stringent (few microradians) tolerances for the angular stability of mirrors and grating assemblies are required for FEL operation. Laser-based optical feedback techniques employing active alignment systems are proposed in Ref. [32] to control the alignment of delicate optical systems in the FEL amplifier at the TESLA TTF facility. Experience in aligning optical elements separated by long $(65 \mathrm{~m})$ distances are also reported. OTR screens and laser-based alignment techniques have been adopted for preliminary alignments and final requirements have been met by directly using the VUV SASE FEL radiation.

\section{Digital Signal Processing}

Interest in Digital Signal Processing (DSP) has dramatically increased in the last decade. The use of DSP technologies is the driving force for the hardware performance progress registered when functions once accomplished by analog methods were improved with a digital approach. Factors guiding the decision toward digital implementations are speed, precision, the signal-to-noise ratio and dynamic range. In addition, another key aspect of a DSP approach includes the increased variety of sophisticated processing algorithms as well as a better 
reconfiguration flexibility and control of accuracy requirements. This is often achievable at lower cost.

The implementation of a number of application and diagnostics tools can be conceived as DSP-based:

- Orbit/trajectory acquisition and display;

- Transverse trajectory stability feedback-feedforward;

- Crab cavity amplitude and phase control;

- Beam loss diagnostics;

- Power converters control;

- Phase and amplitude optimization in the superconducting linac.

There is, of course, interest in standardization, in the as a choice of a DSP platform (hardware and software) covering the whole project and, if possible, common to other domains inside the facility.

\section{REFERENCES}

[1] J. N. Corlett et al., Initial Feasibility Study of a Dedicated Synchrotron Radiation Light Source for Ultrafast X-Ray Science, LBNL-48171, Jan. '02.

[2] G. Dattoli et al., Electron beam properties and impedance characterization for storage rings used for free electron lasers, NIM A 471 (2001) 403-411.

[3] F. - J. Decker et al., Short High Charge Bunches in the SLAC Linac, Proc. $20^{\text {th }}$ Int. Linac Conf. (Linac 2000), 21-25 August 2000. SLAC-PUB-8555, Aug. 2000.

[4] M. Placidi, Preliminary Considerations on Diagnostics and Instrumentation Requirements for the LBNL Femtosource Project, LBNL, April 2002, CBP Tech Note- 277.

[5] M. Nakamura et al. Evaluation of electron bunch shapes using the spectra of the coherent radiation, NIM A 475 (2001) 487-491.

[6] J. D. Jackson, Classical Electrodynamics, Wiley, New York 1975, $2^{\text {nd }}$ Ed., p.638.

[7] J. D. Jackson, Classical Electrodynamics, Wiley, New York 1975, $2^{\text {nd }}$ Ed., p.685.

[8] Sung Oh Cho et al., Time-resolved measurement of electron beam emittance and energy spread with optical transition radiation, NIM A 407 (1998) 359-363.

[9] X. Artru et al., Resolution power of optical transition radiation: Theoretical considerations, NIM B 145 (1998) 160-168.

[10] Y. Shibata et al., Phys.Rev. E 52 (1995) 6787.

[11] A. Yariv, in Quantum Electronics, Wiley, New York 1975, $2^{\text {nd }}$ Ed., Chap. 14.

[12] R. Jung et al.,The LHC 450 to 7TeV Synchrotron Radiation Profile Monitor using a Superconducting Undulator, Proc. BIW02, Brookhaven, N.Y., 6-9 May, 2002.

[13] A. H. Lumpkin et al., Optical techniques for electron-beam characterizations on the APS SASE FEL project, NIM A 429 (1999) 336-340.

[14] B. Feng et al., Electron bunch shape measurement using coherent diffraction radiation, NIM A 475 (2001) 492-497. 
[15] J.Matheson, Nonlinearities in the response of a beam position monitors for the LEP spectrometer, NIM A 466 (3) (2001) 436-447.

[16] R. H. Miller et al., Proc. $12^{\text {th }}$ Int. Conf. on H. E. Accelerators, Batavia, IL, 1983.

[17] A. Jansson and D.J.Williams,A new Optimised Quadrupole Pick-up Design Using Magnetic Coupling, NIM A 479 (3) (2002) 233.

[18] A. H. Lumpkin et al., Electron beam bunch length characterization using incoherent and coherent transition radiation on the APS SASE FEL project, NIM A 445 (2000) 356-361.

[19] A. H. Lumpkin et al., Development of a coherent transition radiation-based bunch length monitor with application to the APS RF thermionic gun beam optimization, NIM A 475 (2001) 476-480.

[20] A. H. Lumpkin, N.S.Sereno and D. W. Rule, First measurements of subpicosecond electron beam structure by autocorrelation of coherent diffraction radiation, NIM A 475 (2001) 470475.

[21] T. Hara et al., Observation of hard X-ray pulses with a highly sensitive streak camera, NIM A 467-468 (2001) 1125-1128.

[22] A. Lumpkin, Potential applications of a dual-sweep streak camera system for characterizing particle and photon beams of VUV, XUV and X-ray FELs, NIM A 375 (1966) 460-464.

[23] M. Geitz et al., Sub-picosecond bunch length measurement at the TESLA test facility, NIM A 445 (2000) 343-347.

[24] X. Yan et al., Application of electro-optic sampling in FEL diagnostics, NIM A 475 (2001) 054-508.

[25] I. Wilke et al., Single-Shot Electron-Beam Bunch Length Measurements, Phys. Rev. Lett. (88) 12, 25 March 2002.

[26] M. S. Zolotorev, G. V. Stupakov, SLAC-PUB 7132, March 1966.

[27] P. Catravas, W. P. Leemans, J. S. Wurtele and MS Zolotorev, Measurement of ElectronBeam Bunch Length and Emittance Using Shot-Noise-Driven Fluctuations in Incoherent Radiation, Phys. Rev. Lett. Vol. 82, No. 26, June 28, 1999.

[28] P. Catravas, E. Esarey and W. P. Leemans, Radiation sources and diagnostics with ultrashort electron bunches, Bull. Am. Phys. Soc. 46,135 (2001).

[29] V. Sajaev, Determination of Longitudinal Beam Profile using Spectral Fluctuations of Incoherent radiation, Proc. EPAC 2000, Vienna, Austria.

[30] P. Tenenbaum et al., Beam-Based Alignment of the Final Focus Test Beam, SLAC-PUB95-7058, Dec. 1995.

[31] F. Zimmermann et al., Performance of the 1994/95 SLC Final Focus System, Proc. 16 ${ }^{\text {th }}$ IEEE Particle Accelerator Conference (PAC 95) and International Conference on High Energy Accelerators (IUPAP), Dallas, Texas, 1-5 May 1995. SLAC-PUB-95-6790, June 1995.

[32] B. Faatz et al., Alignment of the optical feedback system of VUV regenerative FEL amplifier at the TESLA test facility at DESY, NIM A 483 (2002) 412-417. 


\section{LASER SYSTEMS}

\section{INTRODUCTION}

Short-pulse laser systems will be instrumental components of the recirculating linac based synchrotron light source for ultrafast $\mathrm{x}$-ray science. Both the machine operation and the scientific measurements at the beamline endstations will rely on short-pulse laser systems that must be integrated and synchronized at various levels of accuracy. The technical requirements for these lasers and how they integrate with the recirculating linac will be an important consideration in developing the conceptual design of the machine.

Lasers will play important roles in two areas of the machine operation. First, the overall machine timing will be determined by a modelocked laser oscillator (Master Oscillator). This laser will be the source of $1.3 \mathrm{GHz}$ signals for the linac and $3.9 \mathrm{GHz}$ signals for the superconducting rf deflecting cavities. Secondly, the linac photo-injector will require a dedicated laser system for driving the photo-cathode.

Lasers will also be widely employed at the beamline endstations, and each of these lasers will depend on the Master Oscillator for timing. The general scientific motivation for the hard x-ray component of the recirculating linac is to apply $\mathrm{x}$-ray techniques in a time-resolved manner in order to investigate structural dynamics on the fundamental time scale on which atoms move, $\sim 100$ fs. The achievable temporal resolution will be dictated by the x-ray pulse duration, the laser pulse duration (at the beamline endstation) and the relative timing accuracy between laser and x-ray pulses. The beamline laser systems will be used for pump-probe measurements in which a laser pulse is used to initiate the dynamics of interest in a sample, and an x-ray pulse (delayed in time) is used to probe the transient sample response. Measurements will be typically done in a repetitive manner, and the dynamics mapped out as a function of the time delay between the excitation pulse and the probe pulse. Time-resolved x-ray measurements at the resolution limit (dictated by either the $\mathrm{x}$-ray or laser pulse duration) will require absolute synchronization between the laser and x-ray pulses. The laser pulses must be delivered to the beamline endstations, and should provide the maximum flexibility to the beamline users in terms of pulse duration, tunability, pulse energy etc.

Figure 17-1 illustrates the general layout of the various laser systems for the recirculating linac. The overall timing for the machine is determined by the Master Oscillator which consists of a passively modelocked femtosecond laser oscillator coupled to a high-stability rf generator, and is described in Chapter 19-Synchronization. The Master Oscillator is the original source for the RF signals required for the linac as well as the original source of seed laser pulses for laser amplifiers at various beamline endstations. The photocathode drive consists of a second laser system (oscillator/amplifier combination) that is slaved to the Master Oscillator. Laser pulses from the Master Oscillator are distributed via an optical transport system to various beamlines. These pulses may be amplified directly at the beamline endstations in order to create laser pulses for sample excitation. Alternatively, seed pulses from the Master Oscillator may be effectively re-generated at the beamline endstations by using a separate modelocked femtosecond laser oscillator (synchronized to the Master Oscillator), followed by a power amplifier. 


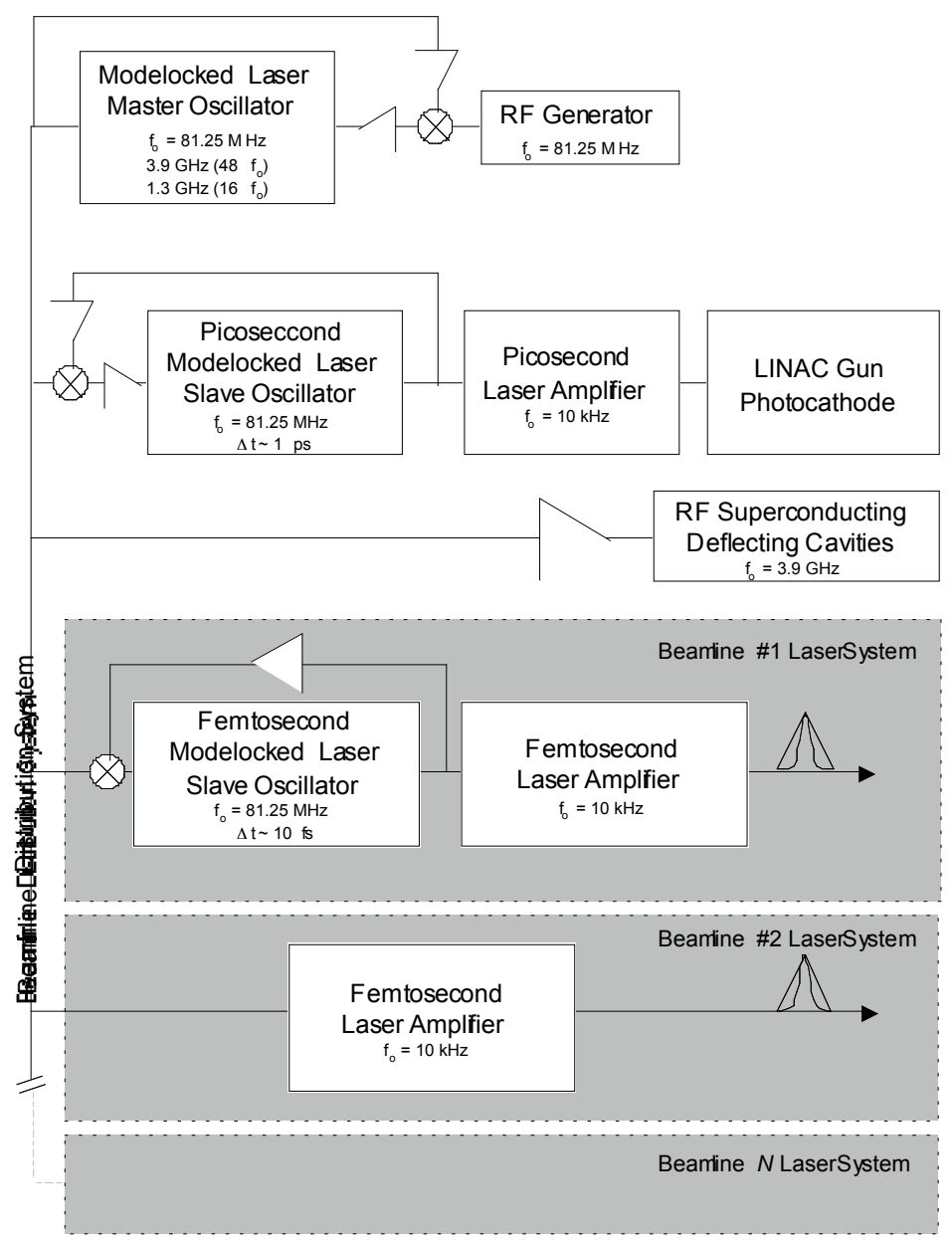

Figure 17-1 Schematic layout of the laser systems.

\section{MASTER OSCILlator}

The Master Oscillator provides the fundamental rf timing for the linac as well as femtosecond seed pulses for the beamline endstations. Thus, timing accuracy and phase stability are the most important criteria in the design of this component. The heart of the Master Oscillator is a passively modelocked femtosecond laser cavity providing optical pulses of less than $100 \mathrm{fs}$ duration. In essence, this laser is a highly stable comb generator. By illuminating a photodiode with output pulses from this laser, one can generate $\mathrm{rf}$ harmonics extending from the fundamental oscillator frequency (cavity frequency $=81.25 \mathrm{MHz}$, round trip time $=12.3 \mathrm{~ns}$ ), up to the bandwidth limit of the photodiode (which can extend well into the GHz range). Thus, this laser is a direct source for all the necessary rf signals for the linac. Because the laser is passively modelocked, the phase noise is substantially lower than that of conventional rf oscillators at frequencies above $\sim 1 \mathrm{kHz}$. The dominant phase noise contribution for such lasers originates typically from mirror motion due to environmental acoustics as well as amplitude noise of the pump laser [1-3]. With advances in stable diode-pump sources, pump laser effects can be largely eliminated [e.g. Coherent Verdi and Spectra Physics Millenia models]. In addition, air 
turbulence effects are eliminated in hermetically sealed cavities, and in modelocked fiber lasers [4,5], both of which are available from commercial vendors [e.g. Coherent Vitesse and IMRA America Femtolite models]. Low frequency acoustic effects and long-term cavity drift can be effectively suppressed by locking the fundamental cavity frequency to a conventional highstability generator [1]. This is accomplished by constructing a phase-locked loop as illustrated in Figure. 17-2, in which the laser cavity acts as a voltage-controlled oscillator by modulating the cavity length with a moving mirror attached to a piezoelectric transducer. Thus, a femtosecond laser phase-locked to a stable rf generator provides phase noise levels which match that of the rf generator at low frequencies (DC to $\sim 1 \mathrm{kHz}$ ), and are substantially better at high frequencies.

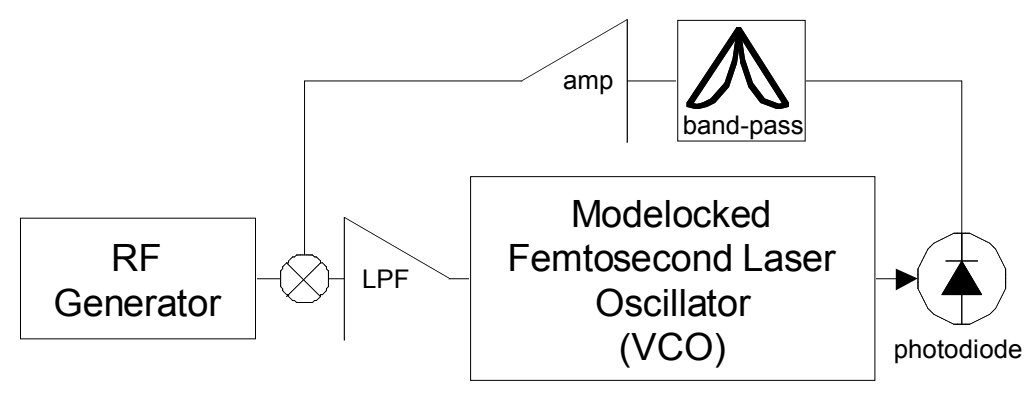

Figure 17-2 Master Oscillator.

Table 17-1 summarizes the essential characteristics of the Maser Oscillator laser. There are several important factors in determining the operating wavelength of this laser. First, most of the beamline laser systems are likely to be based on the solid-state laser media Ti:sapphire which has a peak gain near $800 \mathrm{~nm}$. A Master Oscillator laser operating at this wavelength can provide femtosecond pulses for direct seeding of a Ti:sapphire amplifier at a beamline endstation. A second important consideration is the optical distribution system. This system must be extremely stable, particularly with respect to path length drift, (e.g. a path length drift of only $10 \square \mathrm{m}$ corresponds to a time shift of $30 \mathrm{fs}$ ). One approach is to use free-propagating beams and mirrors. In this case, pointing stability will require an active position feedback system and hermetically sealed (low vacuum) optical transport lines. A second approach is to use a fiber delivery system. With either approach, active monitoring of the path length stability is highly desirable. This may be accomplished by back-reflecting a portion of the pulses from the Master Oscillator to provide a path-length reference. Alternatively, a cw reference laser may be used with interferometric techniques to monitor and control the path length.

One advantage of an optical fiber delivery system is that it completely eliminates any pointing stability problem. However, in a fiber delivery system the nonlinear optical effects and pulse-stretching effects (group-velocity dispersion) must be adequately managed. Nonlinear optical effects might be managed by propagating sufficiently low pulse energies. Group velocity dispersion might be managed by using photonic bandgap fiber in which the group velocity dispersion is balanced by the modal dispersion [6]. Alternatively, by choosing a fiber laser operating at $1.55 \mu \mathrm{m}$ as the Master Oscillator laser, one can take advantage of zero-dispersion fiber that is routinely used for telecommunications applications. 
Table 17-1 General technical specifications for the Master Oscillator.

$\begin{array}{lc}\text { Master Oscillator Laser } & \\ \text { pulse duration }(\mathrm{fs}) & <100 \\ \text { wavelength }(\square \mathrm{m}) & 0.78(1.55) \\ \text { repetition rate }(\mathrm{MHz}) & 81.25 \\ \text { average power }(\mathrm{W}) & \sim 1 \\ \text { phase noise } & <120 \mathrm{dBc} / \mathrm{Hz}\end{array}$

\section{Linac Photocathode Drive Laser}

The drive laser for the linac photocathode will be an independent system with a separate laser oscillator and amplifier. Although the required electron bunch duration from the photocathode gun is $\sim 20 \mathrm{ps,} \mathrm{the} \mathrm{timing} \mathrm{requirements} \mathrm{for} \mathrm{this} \mathrm{system} \mathrm{remain} \mathrm{stringent} \mathrm{as} \mathrm{this} \mathrm{will} \mathrm{be} \mathrm{the} \mathrm{main}$ source of timing jitter of the electron beam with respect to the master oscillator. Stability and reliability are extremely important in order to allow synchronization of pulses produced before the deflecting cavities, and in FEL's.

This Nd:YLF based laser system consists of a passively modelocked diode-pumped laser oscillator generating $10 \mathrm{ps}$ pulses at $1.053 \square \mathrm{m}$. Such oscillators are commercially available from Time-Bandwidth Products (model GE-100). The oscillator will be phase locked to the Master Oscillator (as illustrated in Fig. 17-3) with an rms jitter of less than 1 ps. Pulses from the oscillator will be amplified in a Nd:YLF based regenerative amplifier followed by frequency quadrupling to reach $0.26 \square \mathrm{m}$. Suitable laser amplifiers based on Nd:YLF are commercially available from Positive Light Inc.

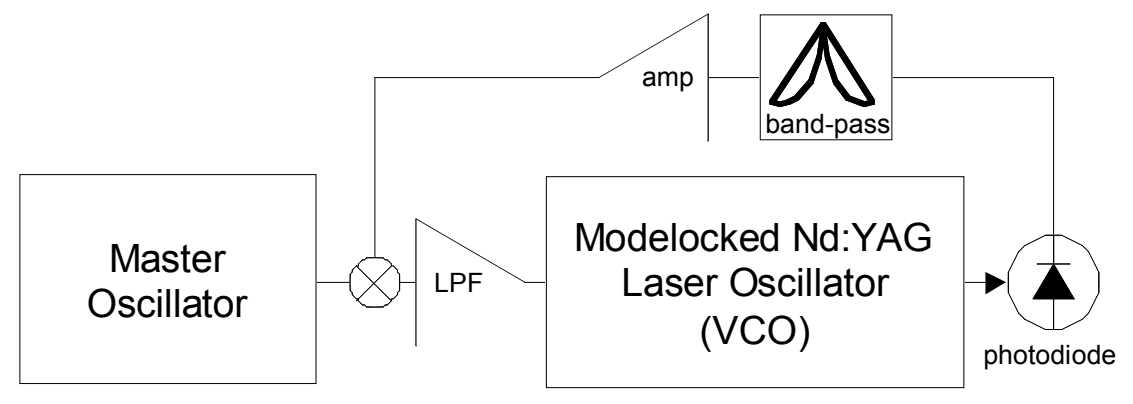

Figure 17-3 Phase locking of Master Oscillator to laser oscillator for photocathode driver.

Table 17-2 General technical specifications for the photocathode drive laser system.

$\begin{array}{lc}\text { Photocathode Drive Laser } & \\ \text { pulse duration }(\mathrm{ps}) & 20 \\ \text { wavelength }(\square \mathrm{m}) & 1.053(0.26) \\ \text { repetition rate }(\mathrm{kHz}) & 10 \\ \text { pulse energy @ } 0.26 \square \mathrm{m}(\mathrm{mJ}) & \sim 0.1 \\ \text { rms jitter w.r.t Master Osc. }(\mathrm{ps}) & <1.0\end{array}$




\section{BEAMLINE LASER SySTEMS}

It is anticipated that separate femtosecond laser systems will be required at each beamline for laser pump/x-ray probe experiments. Furthermore, the requirements for each system will vary depending on the specific research needs of each beamline. Nevertheless, the critical requirements for these laser systems are substantially the same. The most important requirements are timing accuracy and stability with respect to the femtosecond X-rays. Once the synchronization between the Master Oscillator and the femtosecond X-rays is established (as outlined above) the challenge for each beamline laser system is to maintain synchronism with the Master Oscillator. Part of this will rely on the stability of the optical distribution system (either free-space propagation or fiber based) which will deliver a $81.25 \mathrm{MHz}$ train of (low energy, $\sim \mathrm{nJ} /$ pulse) femtosecond optical pulses to each endstation.

Depending on the laser requirements for individual beamline, the laser system may take one of two forms (as illustrated in Figure. 17-1). In the simplest case, the pulses from the Master Oscillator may be use to directly seed a chirped-pulse amplifier (in which the seed pulses are temporally stretched, amplified, and re-compressed). The more general case requires active synchronization of a second laser oscillator (located at the beamline endstation) to the Master Oscillator. This approach is technically more challenging, but considerably more flexible since it is largely independent of the operating wavelength or pulse energy delivered by the Master Oscillator. Synchronization of two independent passively modelocked oscillators has been demonstrated with rms jitter of less than 1 fs (over the $1 \mathrm{~Hz}-160 \mathrm{~Hz}$ frequency range) [2]. Anticipated advances in laser oscillator design and synchronization stability suggest that even better performance will be achieved in the future. The significant advantage of this approach is that it de-couples the requirements of the beamline amplifiers from the Master Oscillator.

The beamline amplifiers may take many forms, possibly depending on the operating wavelength of the Master Oscillator. Nevertheless, amplifier systems are available from several commercial vendors. Standard chirped-pulse amplifiers based on Ti:sapphire are available from Positive Light Inc. and Coherent Inc. for example. Fiber based femtosecond amplifiers are available from IMRA America Inc. Coarse path length differences ( $>10 \mathrm{~ns})$ between laser and Xray pulses are easily compensated by amplifying the appropriate pulse from the Master Oscillator train. Finer path length differences are compensated using a conventional optical delay line. The general requirements of the beamline laser systems are outlined in Table 17-3. A pulse energy of $\sim 1 \mathrm{~mJ}$ should be sufficient for most nonlinear optical techniques (continuum generation, harmonic mixing, parametric amplification etc.) which might be used to generate femtosecond pulses at the appropriate wavelength for sample excitation. Such amplifiers can be readily scaled to even higher pulse energies if necessary.

Table 17-3 General technical specifications for a typical beamline endstation laser system.

$\begin{array}{lc}\text { Beamline Laser (typical) } & \\ \text { pulse duration }(\mathrm{fs}) & <100 \\ \text { wavelength }(\square \mathrm{m}) & 0.8 \\ \text { repetition rate }(\mathrm{kHz}) & 10 \\ \text { pulse energy }(\mathrm{mJ}) & >1.0 \\ \text { rms jitter w.r.t Master Osc. (fs) } & <10\end{array}$




\section{REFERENCES}

[1] D. E. Spence, J. M. Dudley, K. Lamb, W. E. Sleat, and W. Sibbett, "Nearly quantum-limited timing jitter in a self mode-locked Ti:sapphire laser," Opt. Lett., 19, 481-483, 1994.

[2] R. K. Shelton, S. M. Foreman, L. S. Ma, J. L. Hall, H. C. Kapteyn, M. M. Murnane, M. Notcutt, and J. Ye, "Subfemtosecond timing jitter between two independent, actively synchronized, mode-locked lasers," Opt. Lett., 27, 312-314, 2002.

[3] J. Son, J. V. Rudd, and J. F. Whitaker, "Noise characterization of a self-mode-locked Ti:sapphire laser," Opt. Lett., 17, 733-735, 1992.

[4] C. X. Yu, S. Namiki, and H. A. Haus, "Noise of the stretched pulse fiber laser: Part II experiments," IEEE J. Quantum Electron., 33, 660-668, 1997.

[5] S. Namiki, C. X. Yu, and H. A. Haus, "Observation of nearly quantum-limited timing jitter in an all-fiber ring laser,” J. Opt. Soc. Am. B, 13, 2817-2823, 1996.

[6] J. Ranka, R. Windeler, and A. Stentz, "Optical properties of high-delta air-silica microstructure optical fibers," Opt. Lett., 25, 796-798, 2000. 


\section{X-RAY OPTICS AND BEAMLINES}

There are several qualitative differences between the femtosecond linac-based source and an electron storage ring source, concerning x-ray optics and beamlines. The first feature is the position or angle correlation of the electron bunch. In the bending magnets, electrons will have a vertical position-time correlation, in the undulators, a vertical angle-time correlation. These correlations place a requirement on the beamline optics to compress the x-ray pulse or to display the correlation onto a detector. In addition, the average current is low, $10 \mu \mathrm{A}$, and consequently, the power radiated by the undulators is also low, typically $0.2 \mathrm{~W}$. None of the high power optical engineering of the third generation synchrotron radiation sources is required. Silicon or fused silica optics can be used without water cooling. The design of the photon stops in the front ends is also simplified. The elimination of the high average $x$-ray power represents a significant cost savings. Since the $\mathrm{x}$-rays do not have to pass through a storage ring sector arc chamber and front end, the first optic can be close to the source, $\sim 5 \mathrm{~m}$. Finally, a femtosecond laser system will be associated with each beamline.

Briefly, the typical undulator will have the following parameters: length $2 \mathrm{~m}, \lambda_{\mathrm{u}}=1.4 \mathrm{~cm}$ and $\mathrm{B}_{\max }=2 \mathrm{~T}$. Flux curves for the $1^{\text {st }}$ to $11^{\text {th }}$ harmonics are shown in Figure 18-1. The optimal photon energy range of this insertion device is 1.5 to $8 \mathrm{keV}$ at $2.5 \mathrm{GeV}$ beam energy and 2.3 to $12 \mathrm{keV}$ at $3.1 \mathrm{GeV}$. Lower photon energies can be conveniently accessed by longer period undulators. In comparison, the present ALS bend magnet beamline 5.3.1 produces fs X-rays with the laser-electron beam modulation technique at an intensity of $10^{5} 1 /(\mathrm{s} 0.1 \% \mathrm{bw})$. The ALS undulator beamline 6.0 in design will provide fs x-rays at a flux of $10^{7} 1 /(\mathrm{s} 0.1 \% \mathrm{bw})$. The femtosource undulator delivers approximately 4 orders of magnitude more flux than the laserelectron beam modulation sources.

The shape of the undulator harmonics is modified by the angular correlation in the electron bunches. Figure 18-2 shows the $5^{\text {th }}$ harmonic calculated using the URGENT code [1] in the presence and absence of the angular correlation. The vertical acceptance is increased in order to accept the larger angular divergence of the case with angular correlation. The angular correlation broadens the undulator harmonic, but the peak flux is unchanged. This result can be understood from the fact that each electron is radiating the same spectrum but with a variation of the on-axis direction. Brightness is, however, decreased by the increase in the angular divergence.

Asymmetrically cut crystals may be used as optical elements in the x-ray pulse compression scheme [2,3]. As a result of the different angles of incidence and diffraction, a crystal may be

oriented to produce a variable path length across the x-ray beam as shown in Figure 18-3. The optical path length difference $\Delta l$ for an asymmetrically cut crystal is given by:

$$
\Delta l=2 \Delta y \sin \theta \sin \alpha / \sin (\theta+\alpha)
$$




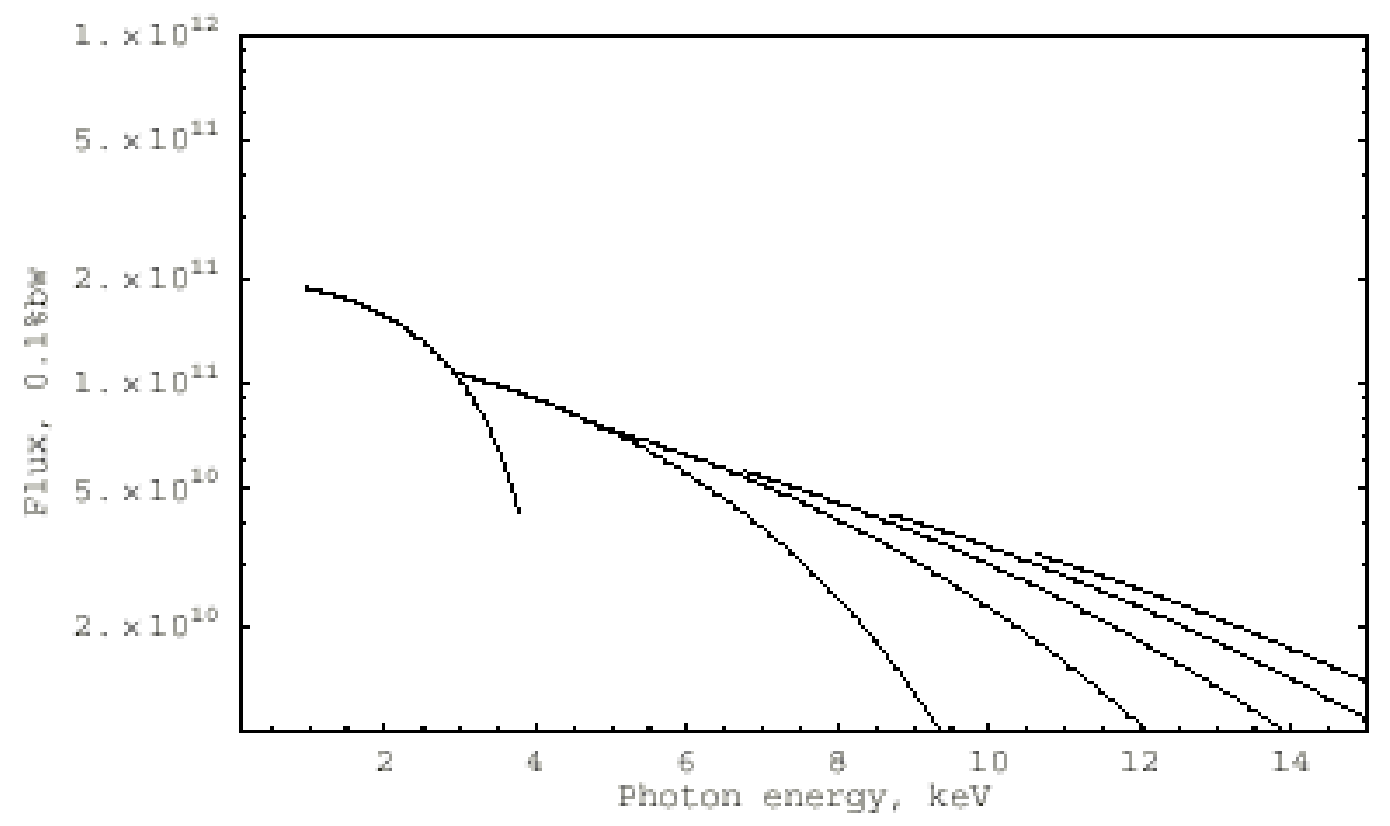

Figure 18-1 Flux of first to eleventh harmonic for the $1.4 \mathrm{~cm}$ period undulator calculated for 2.

$5 \mathrm{GeV}$ electron beam energy.

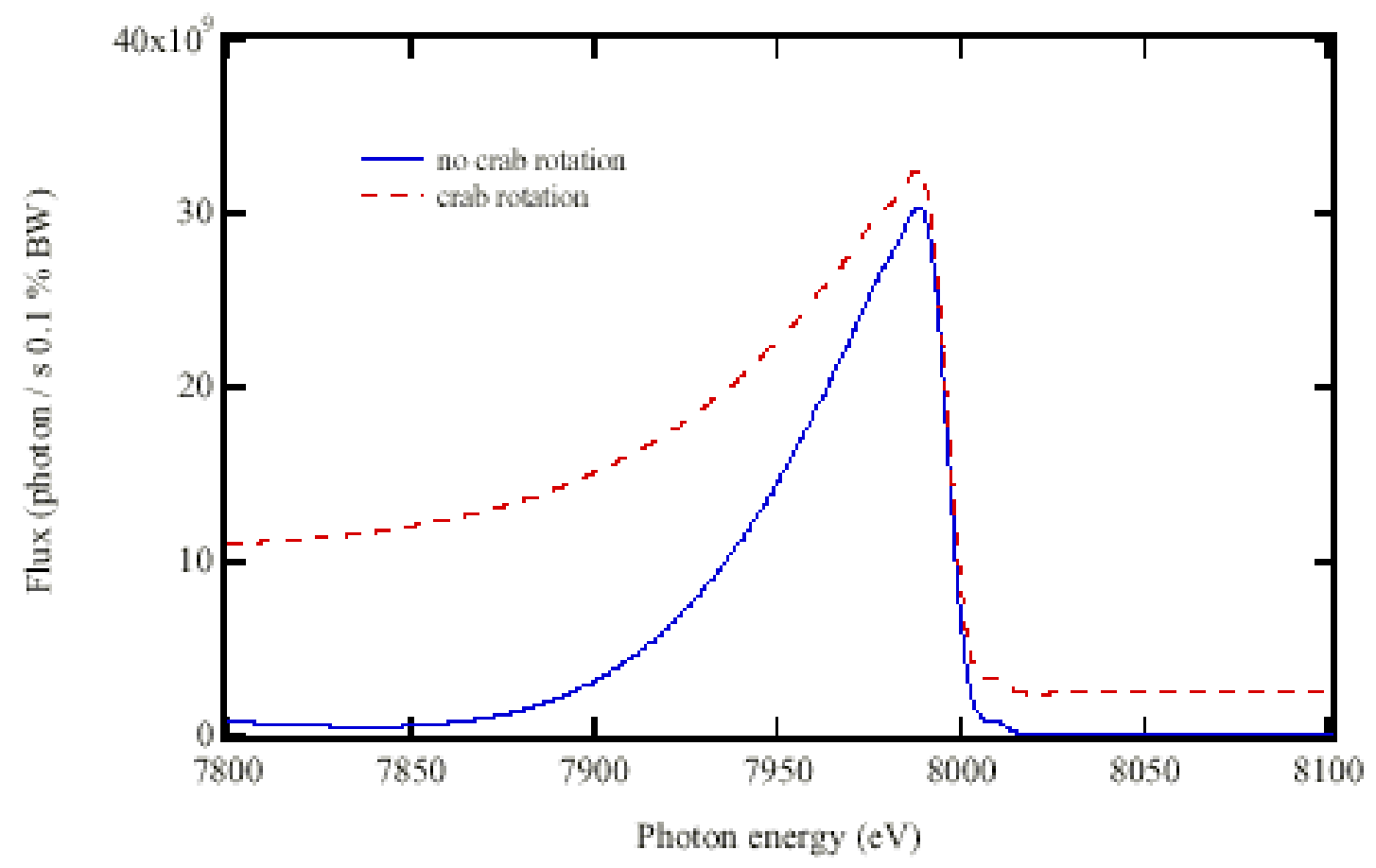

Figure 18-2 The undulator $5^{\text {th }}$ harmonic with and without angular correlation in the electron bunch. 
where $\Delta y$ is the vertical height of the beam at the crystals, $\theta$ is the Bragg angle, $\alpha$ is the asymmetry angle between the Bragg planes and the crystal surface. In Table 18-1, a practical example is given of $\mathrm{x}$-ray pulse compression with two asymmetrically cut crystals.

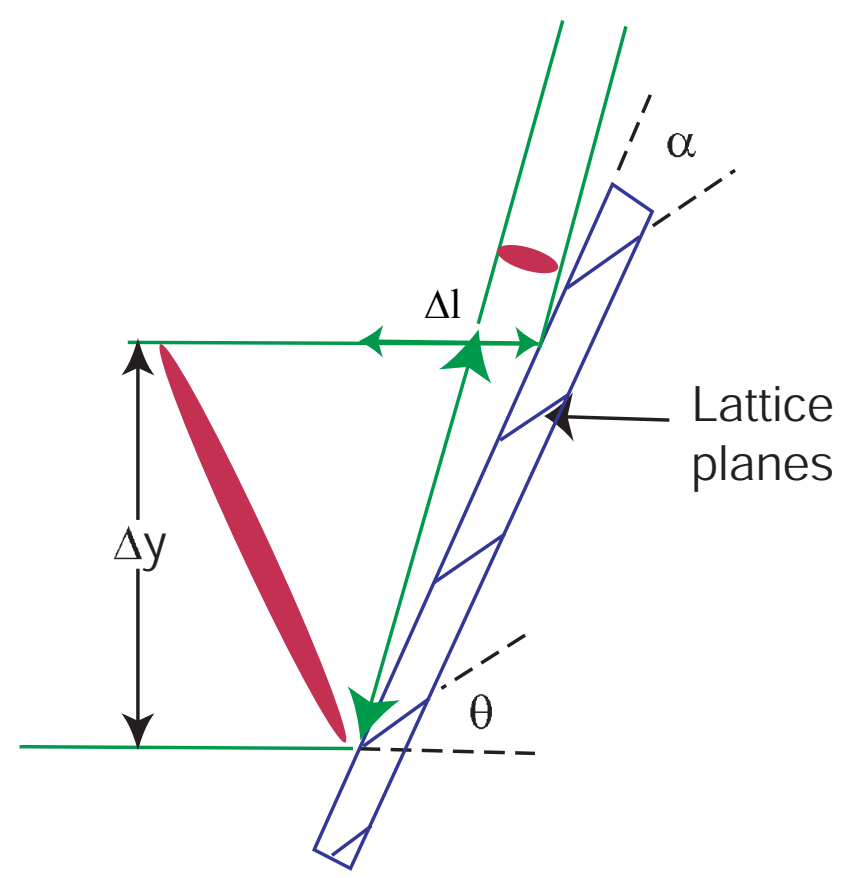

Figure 18-3 Asymmetrically cut crystal with variable optical path length.

Table 18-1 Parameters of two asymmetrically cut crystals for $x$-ray pulse compression.

\begin{tabular}{|c|c|c|c|c|c|}
\hline Crystals & $\lambda$ & $\Delta \mathrm{y}$ & $\theta$ & $\alpha$ & $\Delta l$ \\
\hline $\mathrm{Si}(111)$ & $1.5 \mathrm{~A}$ & $3.8 \mathrm{~mm}$ & $14.309^{\circ}$ & $-3.5^{\circ}$ & $0.6 \mathrm{~mm}(2 \mathrm{ps})$ \\
\hline
\end{tabular}

An issue concerns the scanning of the photon energy while maintaining the $\mathrm{x}$-ray pulse compression. As seen in the Eq. 1, the magnitude of the $\mathrm{x}$-ray pulse compression depends upon the Bragg angle $\theta$. Additional rotation of the crystals about the normal to the Bragg planes, $\phi$, varies the crystal asymmetry in the diffraction plane. In this way the monochromator photon energy may be tuned while keeping the $\mathrm{x}$-ray pulse compression fixed. For a different purpose, a monochromator with asymmetric crystals and additional rotation axes was built and tested at the Advanced Photon Source [4].

Several factors contribute to the duration of the x-ray pulse. First, the angular correlation permits an $\mathrm{x}$-ray pulse compression given by the ratio of the electron bunch rotation by the electron beam divergence. The divergence of the x-rays also gives a contribution, which 
increases with $\lambda^{1 / 2}$. Finally, the penetration of the $\mathrm{x}$-rays into the crystals can broaden the $\mathrm{x}$-ray pulse. But for a low index reflection such as $\mathrm{Si}(111)$, this contribution, given approximately by $\mathrm{N} \lambda$ where $\mathrm{N}$ is the number of diffraction layers, is small. A pulse duration of $50 \mathrm{fs}$ fwhm is achieved at $8 \mathrm{keV}$ photon energy.

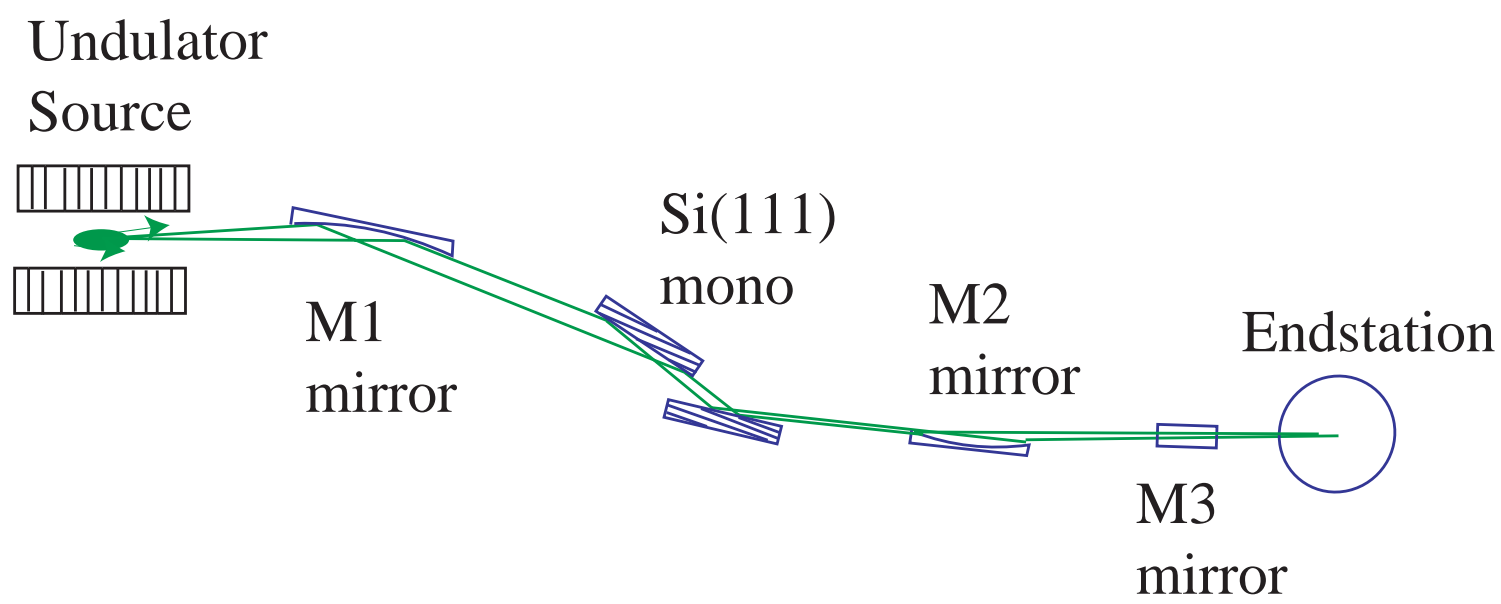

Figure 18-4 Schematic diagram of an undulator beamline with a Si(111) monochromator providing pulse compression.

A schematic layout of an undulator beamline is shown in Figure 18-4. The optical elements are listed in Table 18-2. The source is the electron beam with angular correlation in the small gap undulator. The M1 mirror collimates the x-rays vertically to produce a spatial correlation at a pair of asymmetrically cut $\operatorname{Si}(111)$ crystals. These crystals serve as both monochromator and pulse compressor for the x-rays. At $8 \mathrm{keV}$ photon energy the $\mathrm{Si}(111)$ crystals have a moderate asymmetry factor $b$ of 1.6 and diffract a bandwidth of $0.9 \mathrm{eV}$. The M2 and M3 mirrors act as a Kirkpatrick-Baez pair to refocus the beam into the endstation. The distance from the undulator source to the first mirror is short, approximately $5 \mathrm{~m}$, but this is feasible given the low average beam current of the accelerator.

All three mirrors would be polished as planes and bent to a parabolic or elliptical figure. The tolerance of $1 \mu \mathrm{rad}$ slope error for the M1 and M2 mirrors is at, but not beyond the current stateof-the-art in optical fabrication. The dimensions of the optics, including the length of the M1 mirror, are within the present capabilities of the synchrotron optical vendors. The length of the beamline is $12 \mathrm{~m}$. The photon energy range is from 2 to $11 \mathrm{keV}$.

Raytracing of the undulator beamline has been performed using the XOP software available from the European Synchrotron Radiation Facility [5]. Spot diagrams of the endstation focus are displayed in Figure 18-5. The source dimensions are $390 \mu \mathrm{m}(\mathrm{h}) \times 20 \mu \mathrm{m}(\mathrm{v})$ and divergences are $50 \mu \mathrm{rad}(\mathrm{h}) \times 750 \mu \mathrm{rad}(\mathrm{v})$ total width of the flat-top distribution. The rotation of the electron bunch is included in the vertical divergence. The focus dimensions are $48 \mu \mathrm{m}(\mathrm{h}) \times 55 \mu \mathrm{m}(\mathrm{v})$ and divergences are $0.5 \mathrm{mrad}(\mathrm{h}) \times 0.3 \mathrm{mrad}(\mathrm{v})$ fwhm. The $\mathrm{x}$-ray spot size in the endstation is well matched to a small focal spot for the laser used to excite the sample. The divergence of the 
Table 18-2 A list and description of the optical elements of the undulator beamline.

\begin{tabular}{|c|c|c|c|c|c|c|}
\hline & Type & $\begin{array}{c}\text { Coating and } \\
\text { blank material }\end{array}$ & $\begin{array}{c}\text { Dimensions } \\
(\mathrm{mm})\end{array}$ & $\begin{array}{c}\text { Radius } \\
(\mathrm{m})\end{array}$ & $\begin{array}{c}\text { Incidence } \\
\text { angle }\left(^{\circ}\right)\end{array}$ & $\begin{array}{c}\text { Distance } \\
\text { from } \\
\text { source }(\mathrm{m})\end{array}$ \\
\hline M1 & $\begin{array}{c}\text { Plane } \\
\text { parabolic } \\
\text { mirror }\end{array}$ & $\begin{array}{c}\text { Pt-coated } \\
\text { silicon }\end{array}$ & $650 \times 60$ & 1430 & 89.6 & 5 \\
\hline $\mathrm{X} 1, \mathrm{X} 2$ & Crystal & $\begin{array}{c}\text { Silicon } \\
(111)\end{array}$ & $60 \times 60$ & $\infty$ & $\begin{array}{c}75.6912 \\
\alpha=-3.5\end{array}$ & 6 \\
\hline M2 & $\begin{array}{c}\text { Plane } \\
\text { parabolic } \\
\text { mirror }\end{array}$ & $\begin{array}{c}\text { Pt-coated } \\
\text { silicon }\end{array}$ & $300 \times 25$ & 1430 & 89.6 & 7 \\
\hline M3 & $\begin{array}{c}\text { Plane } \\
\text { elliptical } \\
\text { mirror }\end{array}$ & $\begin{array}{c}\text { Pt-coated } \\
\text { silicon }\end{array}$ & $200 \times 20$ & 339 & 89.6 & 10.667 \\
\hline Endstation & & & & & & \\
\hline
\end{tabular}

$\mathrm{x}$-rays in the endstation is acceptable for spectroscopy and for some x-ray diffraction experiments. At a particular beamline it would be possible to reduce the divergence at the sample with a corresponding increase in the spot size.

At a conceptual level a bend magnet beamline has also been considered. The source is a bend magnet source with a vertical position-time correlation. Here, the coordinate-time correlation of the radiation is used without compressing the x-ray pulse for observations at different time delays. Two Kirkpatrick-Baez mirrors focus the beam onto a thin sample in the transmission geometry. A Varied Line Spacing (VLS) grating spectrograph disperses and focus the $\mathrm{x}$-rays onto a position sensitive detector, where one dimension represents photon energy and the other time. The individual time slices are imaged at the detector. The photon energy range covered would be $100 \mathrm{eV}$ to $2 \mathrm{keV}$. The flux of the bend magnet is less than the undulator source, however entire absorption spectra could be obtained simultaneously. Circular polarization could be provided for magnet samples by accepting radiation above or below the accelerator plane.

Further work is needed in quantitative evaluations of pulse broadening in pulse compression crystals. The detailed undulator beamline design will need to accommodate to space constrains of the shield wall. 


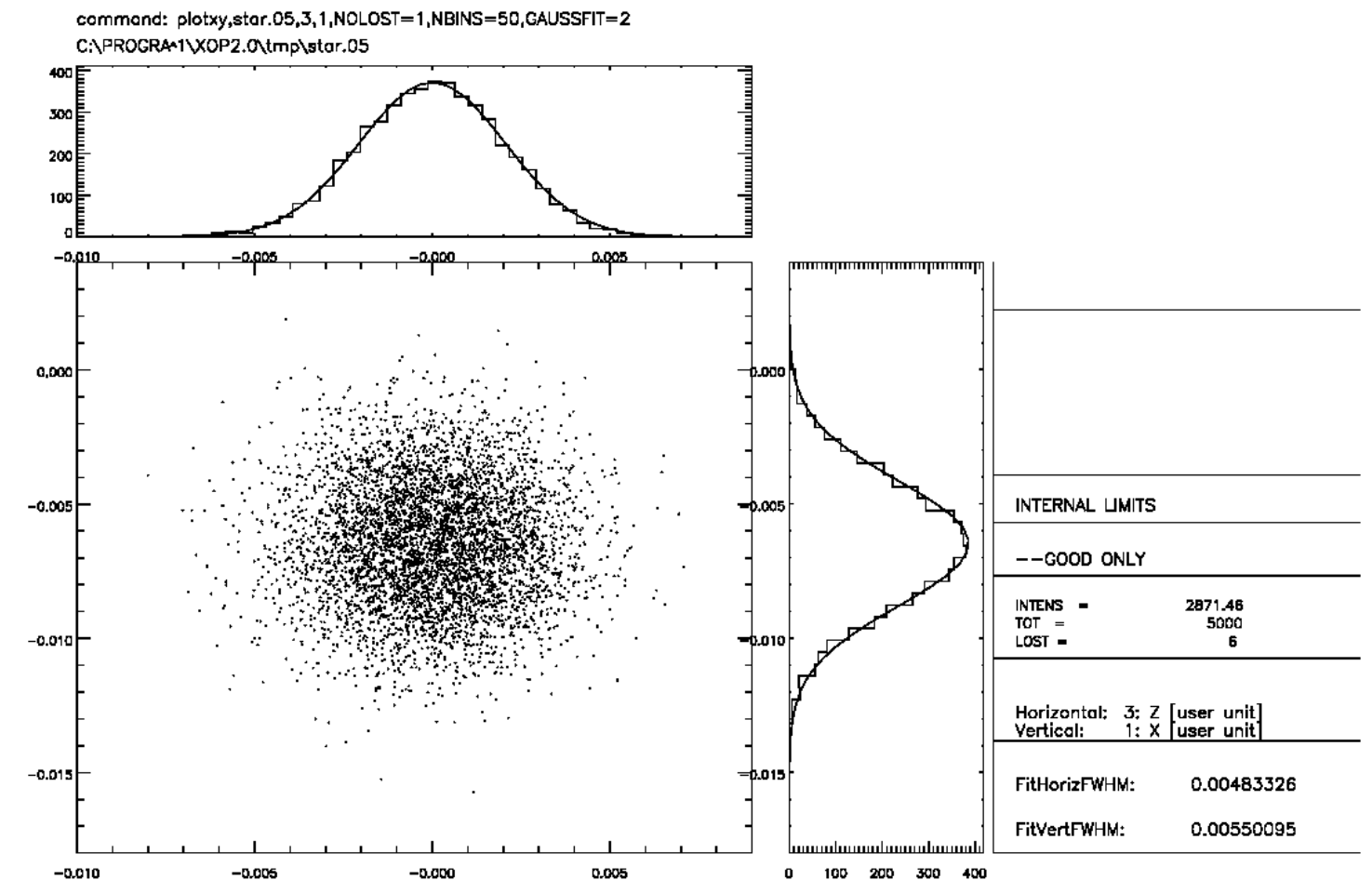

Figure 18-5 Focus dimensions at the endstation of the undulator beamline. Dimensions in cm.

\section{REFERENCES}

[1] R.P. Walker and B. Diviacco, "URGENT-A computer program for calculating undulator radiation spectral, angular, polarization, and power density properties," Rev. Sci. Instrum. 63, 392-395 (1992).

[2] T. Matsushita and H. Hashizume, Handbook of Synchrotron Radiation V.1, ed. E.E. Koch, p.261, North-Holland, Amsterdam (1993).

[3] A. Zholents, P. Heimann, M. Zolotorev, J. Byrd, "Generation of subpicosecond x-ray pulses using RF orbit deflection”, NIM A 425 (1999)385-389.

[4] R.K. Smither, P.B. Fernandez, Nuclear Instrum. and Methods A 347, 313 (1994).

[5] M. Sánchez del Río and R. J. Dejus. " XOP: A Multiplatform Graphical User Interface for Synchrotron Radiation Spectral and Optics Calculations," SPIE Proceedings 3152, 148-157 (1997). 


\section{SYNCHRONIZATION}

\section{INTRODUCTION}

Synchronization between the sample excitation signal and the arrival of the x-ray probe pulse results in stringent timing and stability requirements. For excitation pulses of order $50 \mathrm{fs}$, and xray pulses of similar duration, the synchronization between pulses should be of similar magnitude, 50-100 fs.

Two time regimes may be invoked in discussing synchronization issues - fast jitter on a pulse-to-pulse timescale, and slow jitter occurring over a timescale of many pulses.

Feedback techniques may be applied to stabilize and control the accelerator on both of these timescales. A highly stable master oscillator and timing signal distribution system can be used to phase-lock all lasers and rf systems, minimizing jitter between the emitted x-ray pulse and the sample excitation laser pulse. Fast feedback systems operate around the accelerator rf structures to control timing (via phase feedback in rf structures for example) on short timescales. Slow feedback is derived from the timing error between the x-ray pulse and the experimental excitation pulse, measured at a beamline endstation, and this signal is transported to the fast systems to update setpoints.

Optical signals from synchrotron radiation produced by the electron bunch have the potential to provide an accurate signal with respect to the emitted x-ray photons. Selecting a beam-derived optical pulse from an arc prior to the x-ray production section would allow a time of some $\mu$ s to transport and manipulate the optical pulse before the x-ray pulse arrives at the beamline endstation. This may allow the beam-based optical pulse to seed lasers at the beamline endstations, or to trigger other processes. While this may be seen to have advantages, the production of optical pulses of time duration $\sim 100 \mathrm{fs}$ requires special techniques to compress the synchrotron radiation pulse from the relatively long electron bunch of picosecond duration.

It is likely that a combination of both accelerator control and beam-based signal approaches will produce the most stable facility.

\section{MAster Oscillator}

As described in Chapter 17-Lasers, the Master Oscillator is a passively modelocked femtosecond laser cavity providing optical pulses of less than $100 \mathrm{fs}$ duration. In essence, this laser is a highly stable comb generator. By illuminating a photodiode with output pulses from this laser, one can generate RF harmonics extending from the fundamental oscillator frequency (cavity frequency $=81.25 \mathrm{MHz}$, round trip time $=12.3 \mathrm{~ns}$ ), up to the bandwidth limit of the photodiode (which can extend well into the $\mathrm{GHz}$ range). Thus, this laser is a direct source for all the necessary RF signals for the linac. Because the laser is passively modelocked, the phase noise is substantially lower than that of conventional RF oscillators at frequencies above $\sim 1 \mathrm{kHz}$. The dominant phase noise contribution for such lasers originates typically from mirror motion due to environmental acoustics as well as amplitude noise of the pump laser [1-3]. With advances in stable diode-pump sources, pump laser effects can be largely eliminated [e.g. Coherent Verdi and Spectra Physics Millenia models]. In addition, air turbulence effects are 
eliminated in hermetically sealed cavities, and in modelocked fiber lasers [4,5], both of which are available from commercial vendors [e.g. Coherent Vitesse and IMRA America Femtolite models]. Low frequency acoustic effects and long-term cavity drift can be effectively suppressed by locking the fundamental cavity frequency to a conventional high-stability rf generator [1]. This is accomplished by constructing a phase-locked loop as illustrated in Fig. 191 , in which the laser cavity acts as a voltage-controlled oscillator by modulating the cavity length with a moving mirror attached to a piezoelectric transducer. Thus, a femtosecond laser phaselocked to a stable rf generator provides phase noise levels which match that of the rf generator at low frequencies (DC to $\sim 1 \mathrm{kHz}$ ), and are substantially better at high frequencies. Table 19-1 summarizes the essential characteristics of the Maser Oscillator laser.

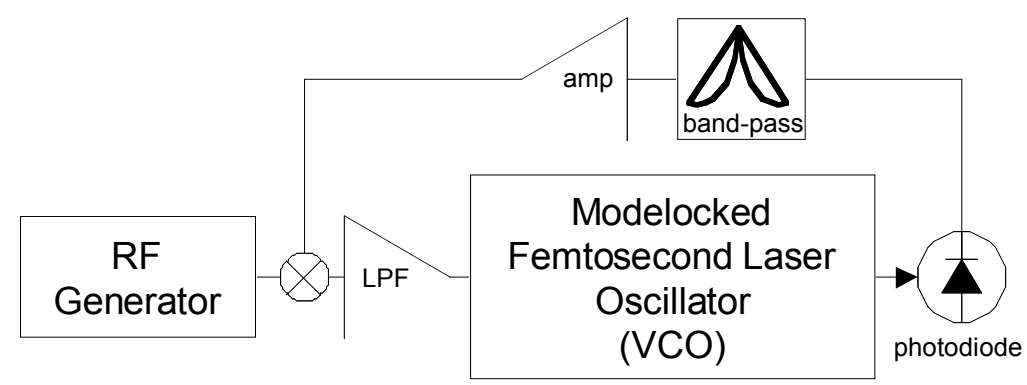

Figure 19-1 Master Oscillator for the recirculating linac source.

Table 19-1 General technical specifications for the Master Oscillator.

$\begin{array}{lc}\text { Master Oscillator Laser } & \\ \text { pulse duration }(\mathrm{fs}) & <100 \\ \text { wavelength }(\square \mathrm{m}) & 0.78(1.55) \\ \text { repetition rate }(\mathrm{MHz}) & 81.25 \\ \text { average power }(\mathrm{W}) & \sim 1 \\ \text { phase noise } & <120 \mathrm{dBc} / \mathrm{Hz}\end{array}$

\section{TIMING DisTRIBUTION SYSTEM}

The optical distribution system must be extremely stable, particularly with respect to path length drift (e.g. a path length drift of only $10 \square \mathrm{m}$ corresponds to a time shift of $30 \mathrm{fs}$ ). One approach is to use free-propagating beams and mirrors. In this case, pointing stability will require an active position feedback system and hermetically sealed (low vacuum) optical transport lines. A second approach is to use a fiber delivery system. With either approach, active monitoring of the path length stability is asssumed. This may be accomplished by backreflecting a portion of the pulses from the Master Oscillator to provide a path-length reference. Alternatively, a cw reference laser may be used with interferometric techniques to monitor and control the path length.

One advantage of an optical fiber delivery system is that it completely eliminates any pointing stability problem. However, in a fiber delivery system the nonlinear optical effects and pulse-stretching effects (group-velocity dispersion) must be managed. Nonlinear optical effects 
may be managed by propagating sufficiently low pulse energies. Group velocity dispersion may be managed by using photonic bandgap fiber in which the group velocity dispersion is balanced by the modal dispersion [6]. Alternatively, by choosing a fiber laser operating at $1.55 \mu \mathrm{m}$ as the Master Oscillator laser, one can take advantage of zero-dispersion fiber that is routinely used for telecommunications applications.

Figure 19-2 illustrates the general layout of the timing systems. The overall timing for the machine is determined by the Master Oscillator which consists of a passively modelocked femtosecond laser oscillator coupled to a high-stability rf generator. The Master Oscillator is the original source for the rf signals required for the linac as well as the original source of seed laser pulses for laser amplifiers at various beamline endstations. The photocathode drive consists of a second laser system (oscillator/amplifier combination) that is slaved to the Master Oscillator. Laser pulses from the Master Oscillator are distributed via an optical transport system to various beamlines. These pulses may be amplified directly at the beamline endstations in order to create laser pulses for sample excitation. Alternatively, seed pulses from the Master Oscillator may be effectively re-generated at the beamline endstations by using a separate modelocked femtosecond laser oscillator (synchronized to the Master Oscillator), followed by a power amplifier. 


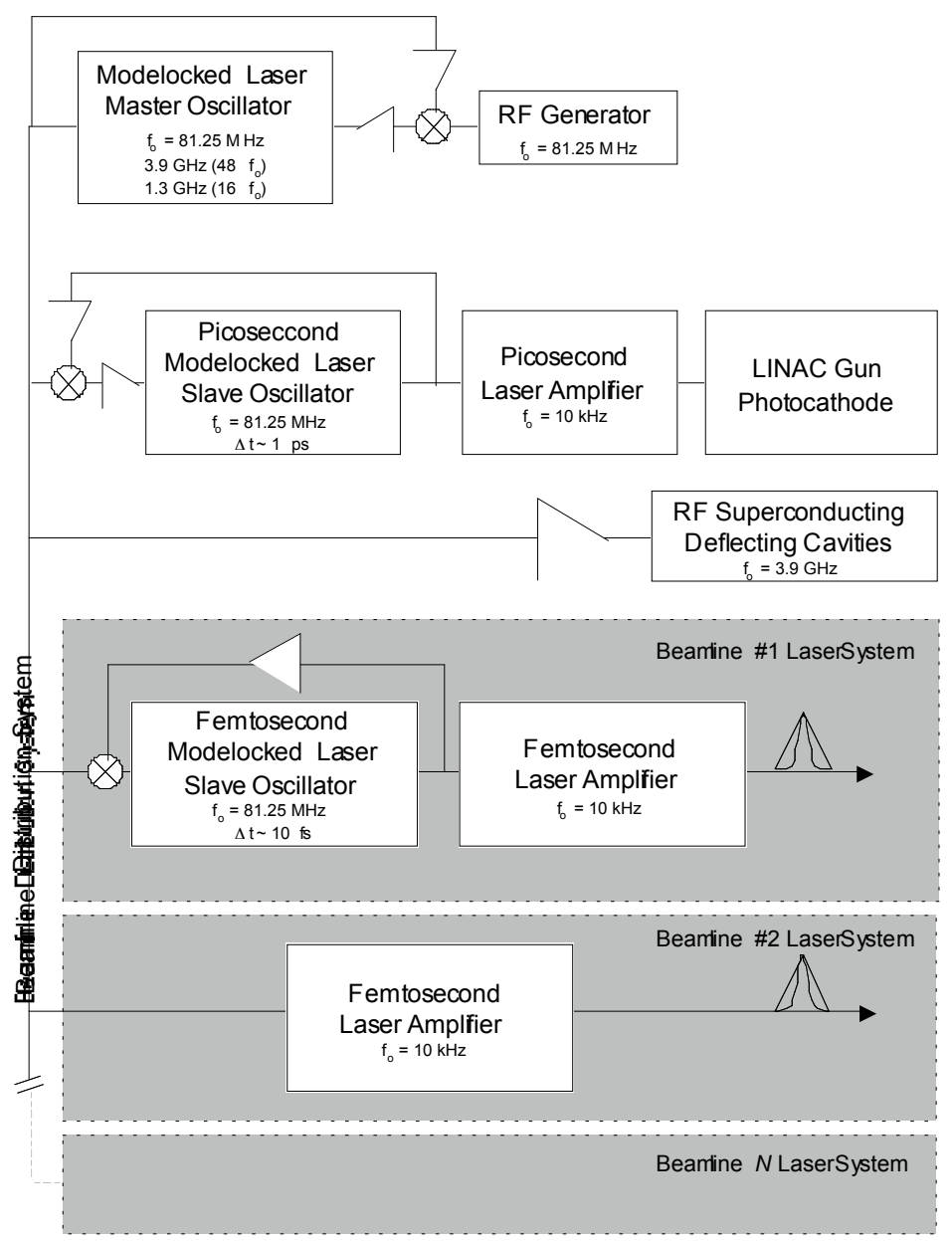

Figure 19-2 Schematic layout of the timing systems.

\section{Delay Lines and Path Lengths}

The path lengths from the Master Oscillator laser to the various beamline endstations will in general not match the path length of the electron beam and x-rays. Thus, delay lines will be required. These delay lines should be as compact as possible and the length kept to a minimum $(\sim 1 \mathrm{~m})$ in order to preserve stability. An important point is that the Master Oscillator laser acts as an extremely stable optical delay line. Beamline users can select any pulse, within a given time window (from the $81.25 \mathrm{MHz}$ train of pulses) for subsequent amplification and sample excitation. This timing accuracy can be understood by considering a time window on the order of $1 \square$ sec. Such a long temporal window should easily encompass any path length discrepancies between laser pulses and x-rays. In such a time window (corresponding to $1 \mathrm{MHz}$ frequency) the motion of the laser cavity mirrors is negligible as illustrated by the following estimation. In $1 \square \mathrm{sec}$ the pulse in the Master Oscillator laser cavity will undergo $\sim 81$ round trips. To maintain a timing accuracy of $30 \mathrm{fs}$, we require that the integrated path length variation (during the 81 round trips) not exceed $10 \square \mathrm{m}$, or $0.12 \square \mathrm{m}$ per round trip on average. Mirror motion of $0.12 \square \mathrm{m}$ 
within the $12.3 \mathrm{nsec}$ round-trip time corresponds to a mirror velocity of $10 \mathrm{~m} / \mathrm{sec}$, which would require a force well beyond anything that could be generated from acoustic disturbances or from the piezoelectric transducer.

\section{Deflecting Cavities}

Our scheme of manipulating the electrons followed by optical pulse compression carries the advantage of providing insensitivity of the x-ray pulse timing at the sample following the crystal optics, to the arrival time of the electron bunch in the deflecting cavities. An electron bunch arriving "synchronously" with the deflecting RF voltage will experience a transverse kick of equal amplitude and opposite direction to the head and tail electrons. Electrons in a bunch arriving early or late with respect to the deflecting voltage zero phase will receive a similar transverse kick dependent on their position within the bunch, but the bunch also receives a net offset kick and the centroid will oscillate afterward. Since the bunch duration is short compared to the $\mathrm{rf}$ period (3.9 $\mathrm{GHz}$ deflecting cavities), the transverse kick to electrons within a bunch remains linear with the same slope. Figure 19-3 shows the perturbation to the electron bunch in the deflecting cavities, resulting in time-position-angle correlation.

In the x-ray crystal optics, the optical path length varies linearly with transverse position on the crystal, and early or late bunches produce radiation that will receive proportionately longer or shorter delays in the optical path, thus remaining synchronous with the deflecting cavity rf phase. Following the asymmetric crystal optics, the time of arrival of the x-ray pulses is the same regardless of the jitter in arrival time of electron bunches at the deflecting cavity. In this way, the temporal jitter of the electron beam is transformed into spatial jitter of the x-ray pulse. Figure. 19-3 gives a pictorial view of this scheme. 


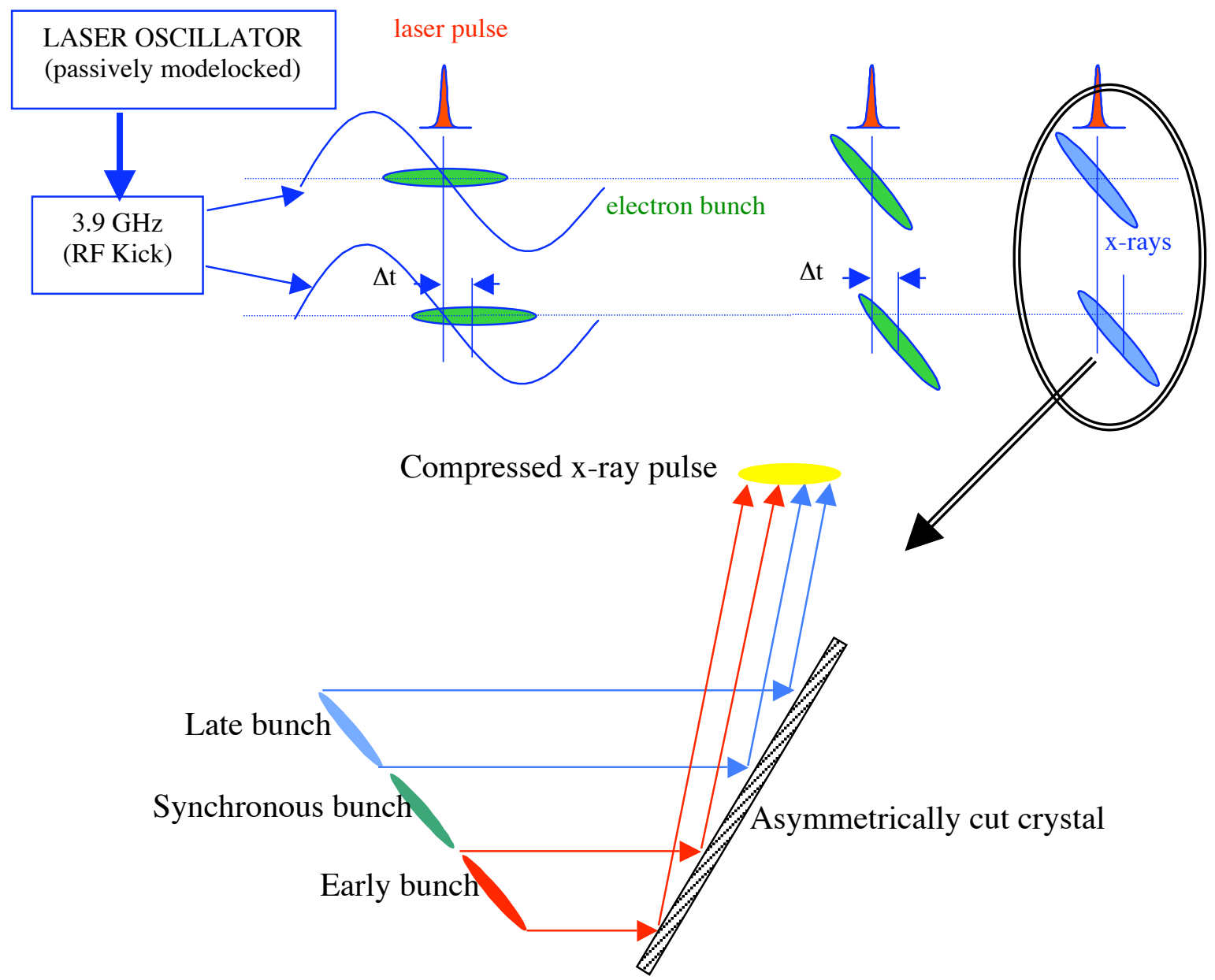

Figure 19-3 The hard $x$-ray production scheme, showing the electron bunch in the deflecting rf field with different arrival times with respect to the cavity phase. Bunches arriving early or late in the cavity still experience a distribution of particles in alignment with those of a bunch arriving in phase with the cavity field. X-rays from such bunches experience the same compression in the $x$-ray optics, resulting in improved timing stability, but with a geometric translation of the x-ray pulse on the sample.

\section{Low-level rf system for the superconducting deflecting cavities}

As described above, with the deflecting cavity locked to the laser oscillator, stability of the xray pulse will depend on the phase stability of the deflecting cavity voltage. The low-level rf system for the superconducting deflecting cavities is instrumental in determining the final synchronization between the beamline lasers and the femtosecond hard x-rays. The rf drive signal at $3.9 \mathrm{GHz}$ is derived directly from the Master Oscillator via illumination of a photodiode and subsequent electronic amplification. Since the fundamental rf signal at its source is absolutely locked to the laser pulses from the Master Oscillator, maintaining this synchronization is largely an issue of feedback control of phase while minimizing the electronic noise from the rf amplifiers and isolating (or actively damping) the deflecting cavities from external acoustic perturbations. 
A 10 fs timing error is equivalent to $0.014^{\circ}$ in phase at $3.9 \mathrm{GHz}$, and we require control at this level. Resolution of 1 fs requires measurement to $2.44 \times 10^{-5} \mathrm{rad}$, or one part in 41,000 . A 16bit DAC offers the required resolution. The system bandwidth of approximately $100 \mathrm{~Hz}$ allows ample time for signal processing.

The superconducting cavity has an external bandwidth requirement of approximately $100 \mathrm{~Hz}$ to allow feedback control of phase and amplitude required due to cavity resonant frequency perturbations arising from microphonically induced changes in cavity geometry.

A photodiode or similar optical-electrical transducer illuminated by the Master Oscillator signal can be attached to an electrical band-pass filter at $3.9 \mathrm{GHz}$, to provide a stable drive signal. At least one isolation stage is needed, either low noise active or a circulator, before this signal is used for measurement and control of cavity fields. Figure 19-4 shows a schematic of the deflecting cavity rf system electronics.

Designing for low jitter really means designing for low noise. Over time spans of 10-100 ms (100 to 1000 pulses), temperature changes and 1/f effects become important, and timing error between the x-ray pulse and the experimental excitation pulse, measured at a beamline endstation will provide a (digital) correction signal for cavity phase over this time period. The cavity control system only has to keep the accelerator stable long enough to get good signal/noise out of the pump laser/x-ray pulse relative timing measurements, and for those results to propagate through networks and software filters to update the digital setpoints.

Numerology of the timing frequencies is important. A simple integer scaling from the Master Oscillator to RF frequencies is probably bad, as that places the output of the mixers at DC. Quarter integer scaling places the output frequency at about $20 \mathrm{MHz}$ for both the 1.3 and 3.9 GHz cavities, which would provide traditional I/Q/-I/-Q output from an $80 \mathrm{MSample} / \mathrm{s}$ ADC. Figure 19-2 shows the deflecting cavity rf system schematic diagram.

Although 16-bit $80 \mathrm{MSample} / \mathrm{s}$ ADCs do not exist at the time of writing, currently available parts (AD6645, 14-bit, $75 \mathrm{~dB}$ SNR at $15 \mathrm{MHz}$ and $80 \mathrm{MSample/s)} \mathrm{come} \mathrm{close} \mathrm{enough} \mathrm{to} \mathrm{allow}$ meaningful tests and future developments may be expected to provide for our requirements. 


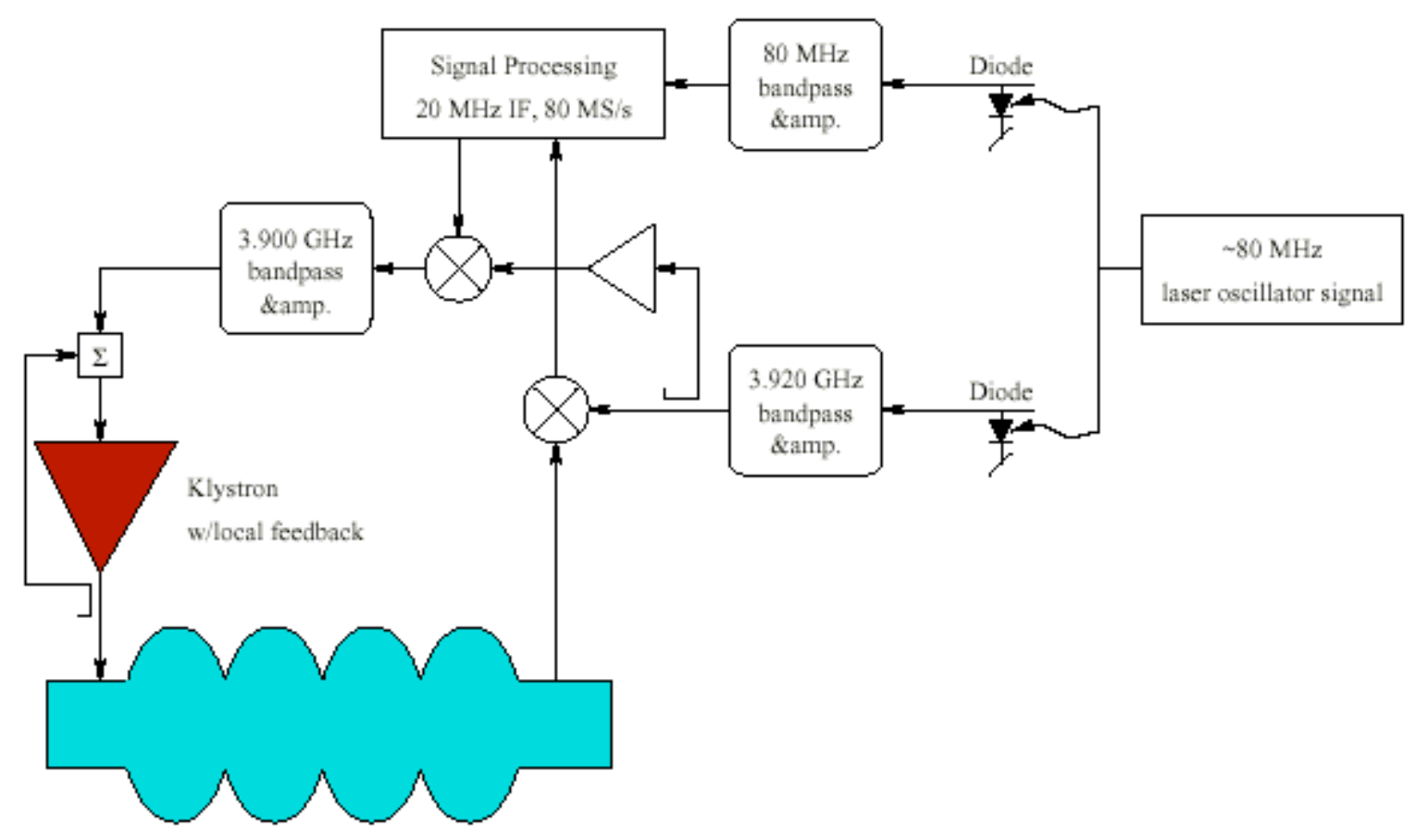

Figure 19-4 RF systems control schematic for the deflecting cavities.

We estimate the thermal noise in the system by assuming a room temperature (T) amplifier with $3 \mathrm{~dB}$ noise figure $(\mathrm{NF})$ and $30 \mathrm{~dB}$ gain $(\mathrm{G})$, bandwidth $(\Delta \mathrm{f}) 100 \mathrm{~Hz}$. The noise power

$$
P_{\text {thermal }}=k T \square f N F G
$$

is then $8 \times 10^{-16} \mathrm{~W}$ or $2 \times 10^{-7}$ volts in $50 \Omega$ ( $\mathrm{k}$ is the Boltzmann constant). A $7 \mathrm{~dB}$ NF mixer would contribute significantly less noise. A resolution of $1 \mathrm{fs}$ requires that the signal equivalent to a $1 \mathrm{fs}$ phase change be greater than the noise level, and we find that the cavity signal amplitude must be greater than approximately $10 \mathrm{mV}$. Such a signal level may readily be obtained from a cavity probe. A similar analysis shows that thermal noise in the photodiode and rf drive chain is not expected to prove problematic.

\section{Low-level rf system for the photocathode rf gun}

Since the bunch compression scheme transforms energy jitter from the injector into timing jitter in the main linac, the launch phase of the electron bunches must be controlled to stabilize energy jitter to an acceptable level. While the rf signal derived from the Master Oscillator is expected to be stable to $\sim 100 \mathrm{fs}$, a low-level rf system similar to that shown in Figure 19-4 may be applied to control the launch phase of the photocathode gun rf fields with respect to the photocathode laser pulse. 


\section{OPTICAL BEAM-BASED SIGNALS}

Two concepts are shown here for measuring the slow changes in electron bunch timing with respect to the Master Oscillator. These signals may be used to update the setpoints for the fast feedback systems described above. In addition, optical signals produced by the beam may be used to seed experimental pump lasers.

The first concept involves preparing a $\sim 100$ fs optical pulse from the synchrotron radiation emitted in a dipole bend magnet of the photon production section. With the electron beam displaced as described in Chapter 1-Overview, the optical pulse may be compressed in an echelle reflection grating to provide a short-pulse timing signal. An optical correlation technique may be used to provide an error signal and update the fast feedback system setpoints. Figure 19-5 shows a schematic of the technique.

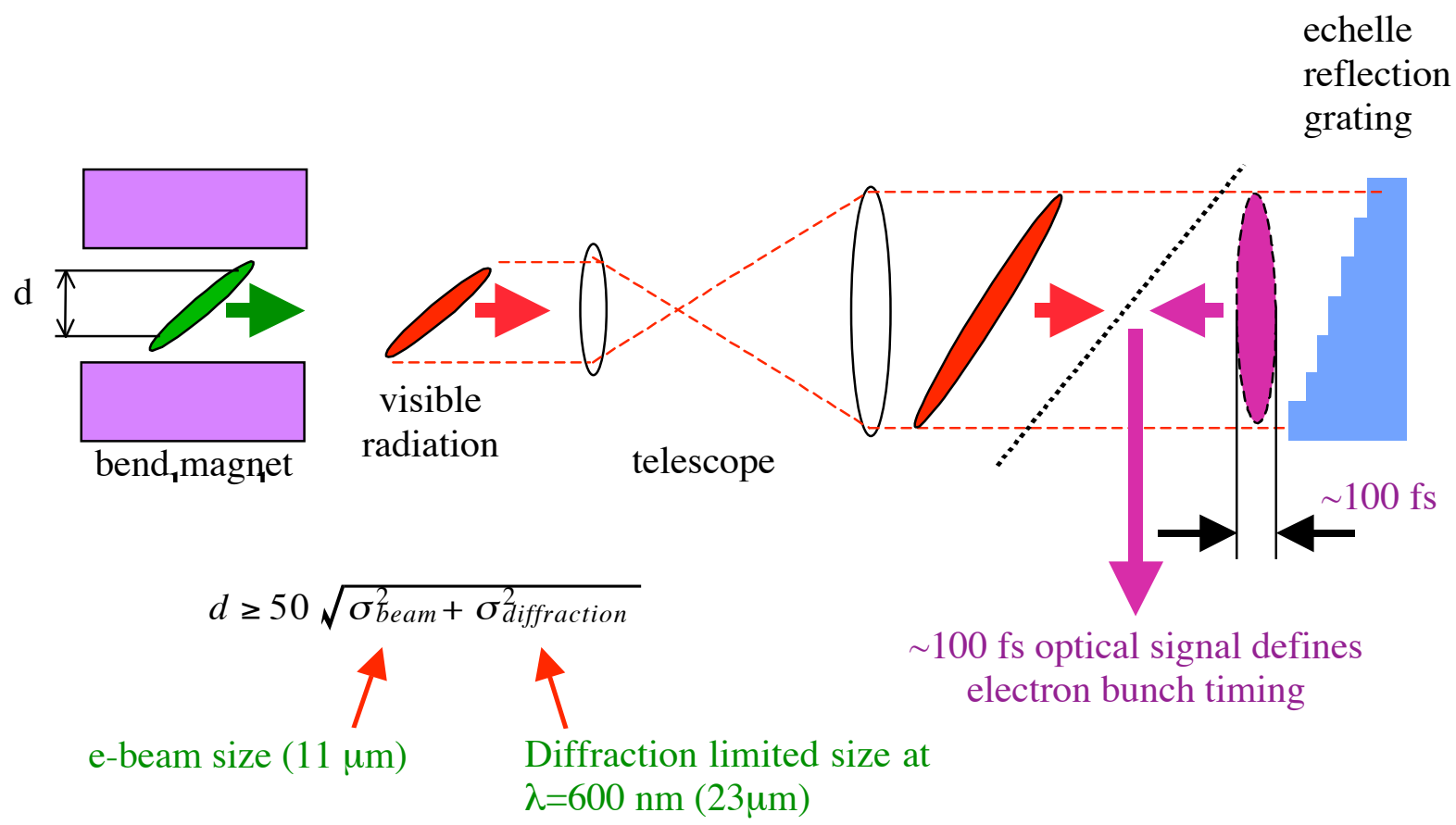

Figure 19-5 Concept for beam-based optical timing pulse production.

Another concept involves optical mixing of the synchrotron radiation pulse and a chirped optical laser pulse to provide timing information from the spatial position of the mixed signal from a crystal. A chirped laser pulse overlaps the short synchrotron radiation pulse, and the output signal detected on a ccd or similar array. The spatial position of the signal will be a function of the color of the laser pulse at which the short synchrotron radiation pulse enters the system, by virtue of the refractive index variation with wavelength. The concept is shown schematically in Figure 19-6. 


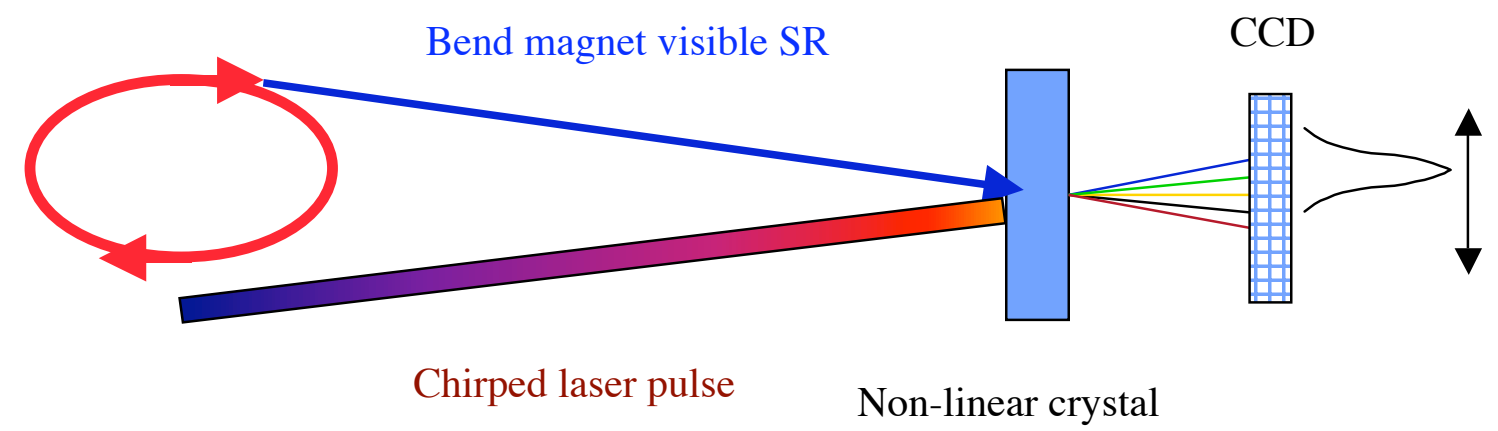

Figure 19-6 Concept for beam-based optical timing pulse production.

\section{REFERENCES}

[1] D. E. Spence, J. M. Dudley, K. Lamb, W. E. Sleat, and W. Sibbett, "Nearly quantum-limited timing jitter in a self mode-locked Ti:sapphire laser," Opt. Lett., 19, 481-483, 1994.

[2] R. K. Shelton, S. M. Foreman, L. S. Ma, J. L. Hall, H. C. Kapteyn, M. M. Murnane, M. Notcutt, and J. Ye, "Subfemtosecond timing jitter between two independent, actively synchronized, mode-locked lasers," Opt. Lett., 27, 312-314, 2002.

[3] J. Son, J. V. Rudd, and J. F. Whitaker, "Noise characterization of a self-mode-locked Ti:sapphire laser," Opt. Lett., 17, 733-735, 1992.

[4] C. X. Yu, S. Namiki, and H. A. Haus, "Noise of the stretched pulse fiber laser: Part II experiments," IEEE J. Quantum Electron., 33, 660-668, 1997.

[5] S. Namiki, C. X. Yu, and H. A. Haus, "Observation of nearly quantum-limited timing jitter in an all-fiber ring laser," J. Opt. Soc. Am. B, 13, 2817-2823, 1996.

[6] J. Ranka, R. Windeler, and A. Stentz, "Optical properties of high-delta air-silica microstructure optical fibers," Opt. Lett., 25, 796-798, 2000. 


\section{BEAM DUMP}

A beam dump for the continuous absorption of the electron beam will be located downstream of the photon production arc. It is anticipated that the beam dump assembly will include an upstream bend magnet that directs the electrons towards a water-cooled dump located below floor level. A series of two beam windows, located between the dump and bend magnet, isolate and safeguard the beamline from contamination by the gas evolved from the dump. A partial pressure of argon gas between the windows will be monitored as a means of detecting a vacuum breech. Additional magnets to raster the beam upstream of the windows may be required to prevent excessive localized heating.

As with the other mechanical systems, the beam dump was designed to be compatible with the upgraded machine parameters of $3.1 \mathrm{GeV}$ and $3 \mathrm{nC}$ charge per bunch on a $10 \mathrm{kHz}$ repetition rate $(93 \mathrm{~kW}$ of beam power). The successful design a high power multi-GeV beam dump must take into consideration several factors including:

- cooling requirements

- thermal stresses

- $\quad$ safety - minimal reliance on active components

- radiation shielding

- activation and handling of activated components

- hydrogen generation (water cooled dumps) and handling

- fabrication feasibility and cost

- $\quad$ operating cost

This initial study primarily addresses thermal issues with some regard for fabrication feasibility. Estimates of the required shielding, hydrogen generation and safety issues are in progress.

The initial dump design, Figure 20-1, consists of a solid $24 \mathrm{~cm}$ diameter central cylinder of POCO graphite surrounded by a $2 \mathrm{~cm}$ thick copper outer layer. The copper is brazed to the graphite to produce a good thermal bond. The carbon/copper assembly resides within a water jacket. The 2-meter length of the carbon center plug is sufficient to absorb most of the energy of the electromagnetic shower. A $5 \mathrm{~cm}$ thick OFHC copper end plug will absorb the low energy tail. The combined radial thickness of copper and carbon is sufficient to absorb more than $99 \%$ of the energy in the transverse direction. Water flowing at a modest rate over the surface of the copper removes $93 \mathrm{~kW}$ of heat on a steady-state basis. 


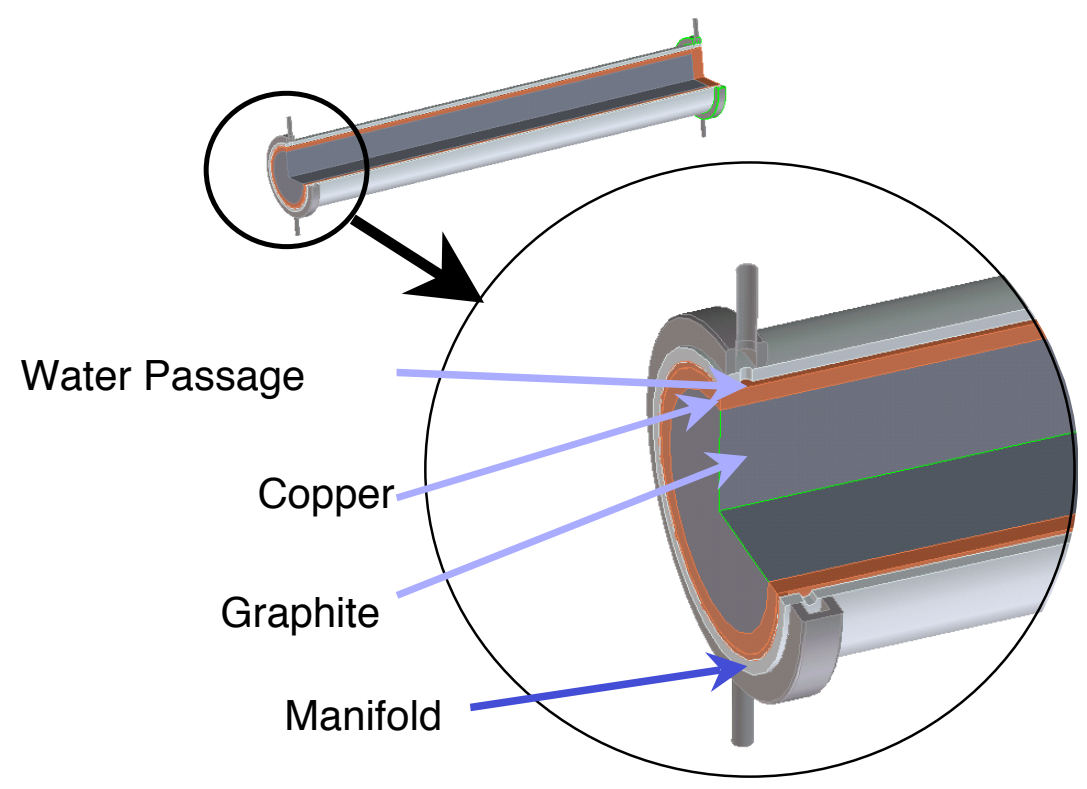

Figure 20-1 Beam absorbing assembly.

Carbon has the advantage of a relatively long (physical) radiation length and excellent thermal conductivity. Pure aluminum has roughly twice the thermal conductivity but less than half of the radiation length of carbon whereas copper has about 4 times the conductivity but less than $1 / 10$ the radiation length. A long physical radiation length leads to a broad longitudinal electromagnetic shower profile and an enhanced radial power deposition. Diffuse longitudinal energy absorption spreads the heat over a greater effective surface area for cooling (at the perimeter) and reduces the peak temperatures. High thermal conductivity allows for a greater radial path length from heated core to cooled surface (for a given peak temperature) and thus a lower power density at the convection boundary due to increase in outer surface area with radius.

A comparison of the power deposition for three candidate absorber materials is shown in Figure 20-2. Power deposition profiles were determined using the formulas for electromagnetic cascades in European Physical Journal C, Particles and Fields [1]. 


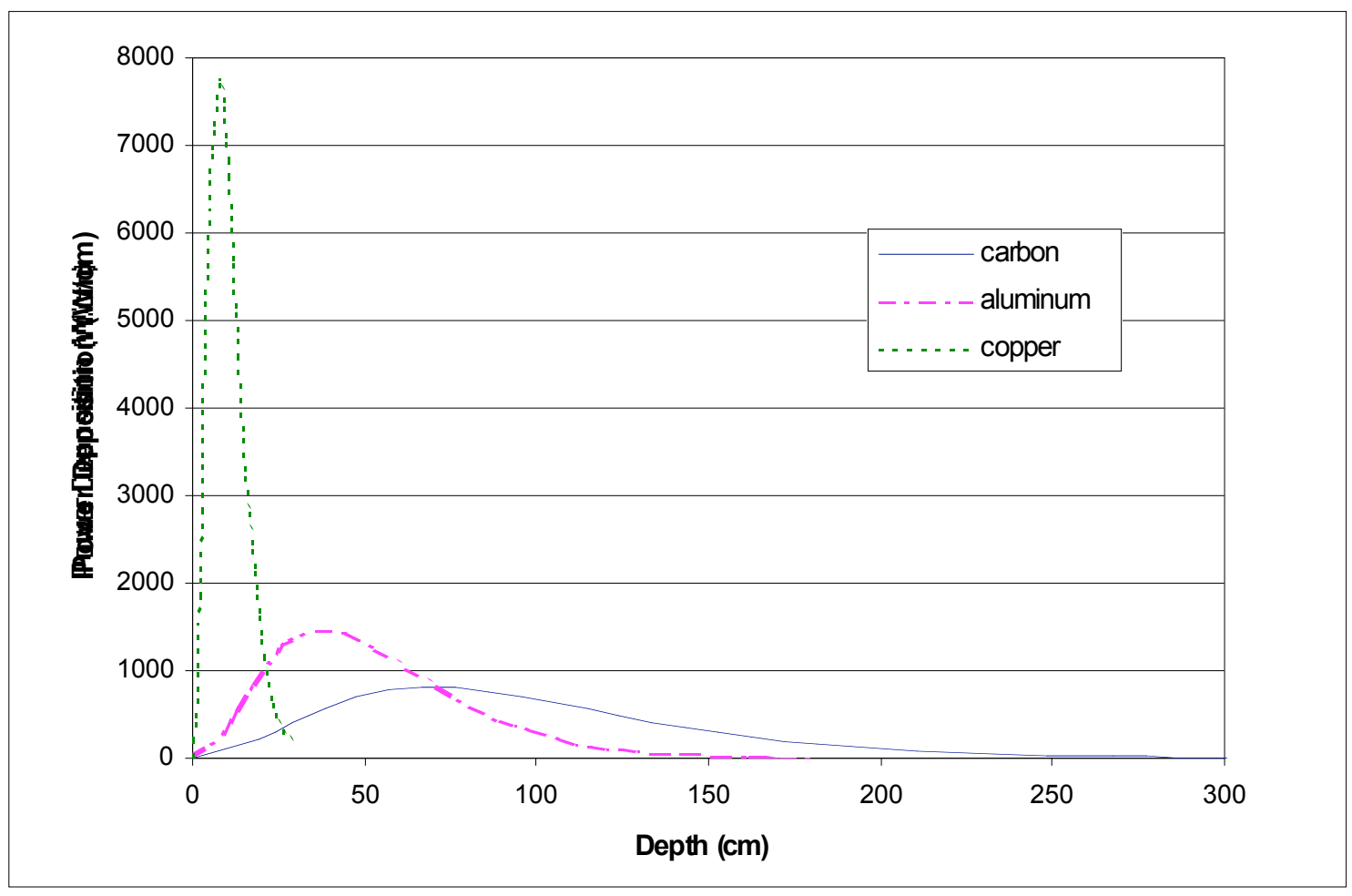

Figure 20-2 Power deposition profiles of an electromagnetic cascade in carbon, aluminum and copper for a $3.1 \mathrm{GeV}, 93 \mathrm{~kW}$ electron beam.

The transverse development of electromagnetic cascades scales with the Moliere radius, $R_{M}$ given by [1]

$$
\mathrm{R}_{\mathrm{M}}=\mathrm{X}_{0} \mathrm{E}_{\mathrm{S}} / \mathrm{E}_{\mathrm{C}}
$$

where $E_{S}=21.2 \mathrm{MeV}, \mathrm{Z}=$ atomic weight, $X_{o}$ is the radiation length, and

$$
\mathrm{E}_{\mathrm{C}}=\frac{610 \mathrm{MeV}}{\mathrm{Z}+1.24}
$$

The critical energy, $E_{C}$, is the energy at which the ionization loss per radiation length is equal to the electron energy [2]. Approximately $90 \%$ of the energy is contained within one $R_{M}$ and $99 \%$ is contained within $3.5 R_{M}$. For copper $R_{M}=1.5 \mathrm{~cm}$ and for carbon $R_{M}=4.8 \mathrm{~cm}$. To approximate the transverse power deposition, a single Gaussian distribution with $\square=R_{M} / 1.65$ (90\% of the swept volume is contained within a radius of $R_{M}$ ). Setting the area under the transverse profile equal to the power dissipation at a given depth of absorber gives the volumetric power deposition at any point in the absorber volume. The actual distribution starts as a narrow core and broadens as the cascade develops so the assumed constant transverse profile underestimates the volumetric power density over the initial length of absorber. Further refinement in this area is required to determine if the electron beam must be rastered. 
The calculated absorbed power density in carbon peaks at roughly $5 \mathrm{~W} / \mathrm{cm}^{3}$ and falls off radially to less than $0.5 \mathrm{~W} / \mathrm{cm}^{3}$ at a radius of $16 \mathrm{~cm}$. To estimate the temperature rise and thermal stress the power density was applied to an axisymmetric FEA model (ANSYS). The resultant temperature and stress plots are shown in Figures 20-3 and 20-4.

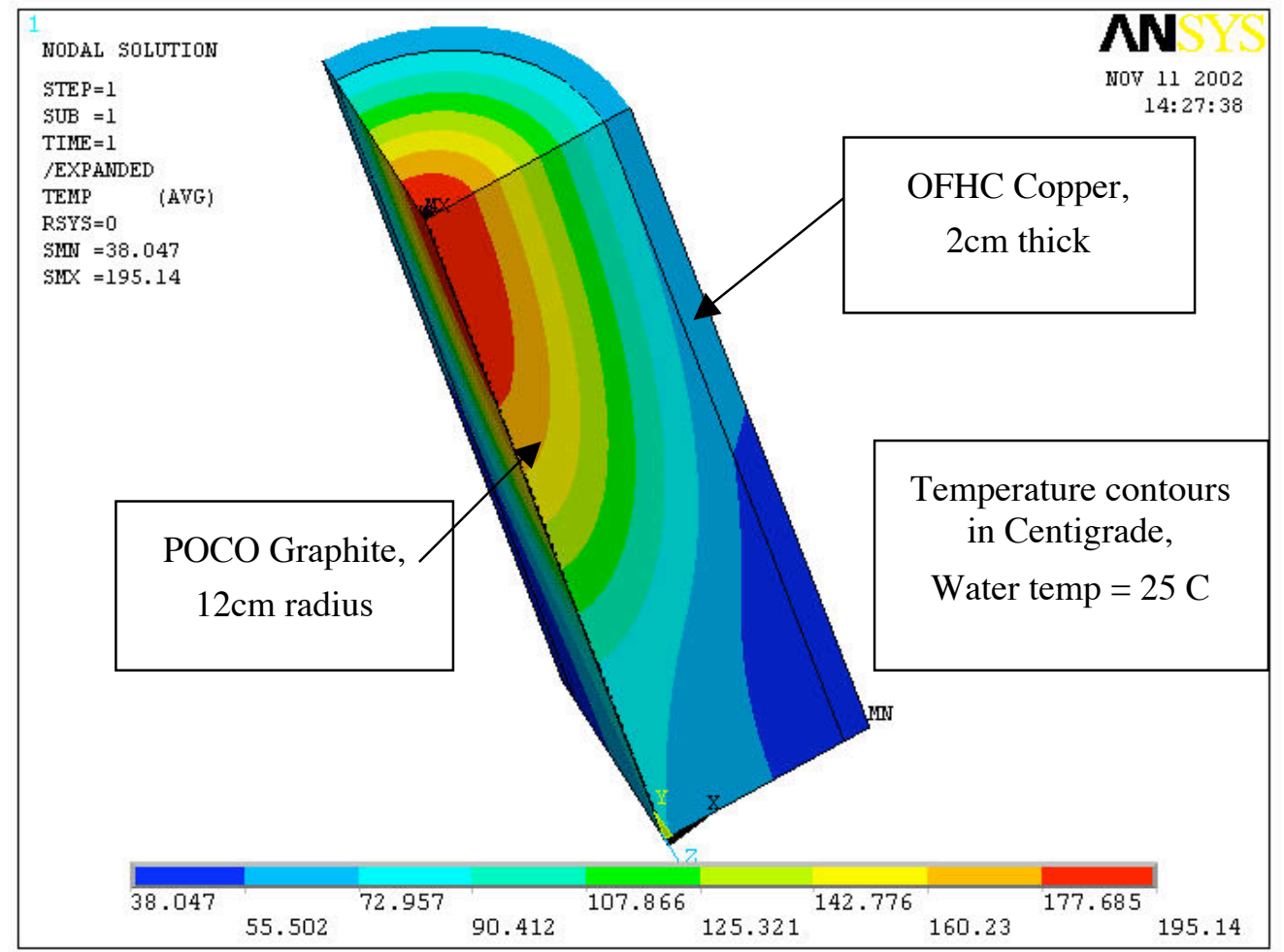

Figure 20-3 Temperature profile for carbon/copper beam dump over the first $75 \mathrm{~cm}$. Convective heat transfer coefficient of $2000 \mathrm{~W} / \mathrm{m}^{2}$-C applied to outer surface.

The model encompasses the first $75 \mathrm{~cm}$ of absorber length, corresponding depth of the energy deposition peak. A symmetry boundary condition was applied at the $x=75 \mathrm{~cm}$ end to take advantage of the near symmetry in the longitudinal energy deposition profile.

The convective heat transfer coefficient of $2000 \mathrm{~W} / \mathrm{m}^{2} \mathrm{C}$ is consistent with $25{ }^{\circ} \mathrm{C}$ water flowing over the surface at $0.37 \mathrm{~m} / \mathrm{s}$. This can be achieved by passing water at $21 / \mathrm{s}$ through a concentric passage having an annular gap of $6 \mathrm{~mm}$. Note that the calculated peak temperature of the graphite is very modest and that the temperature at the convective boundary (in contact with water) is well below boiling. 


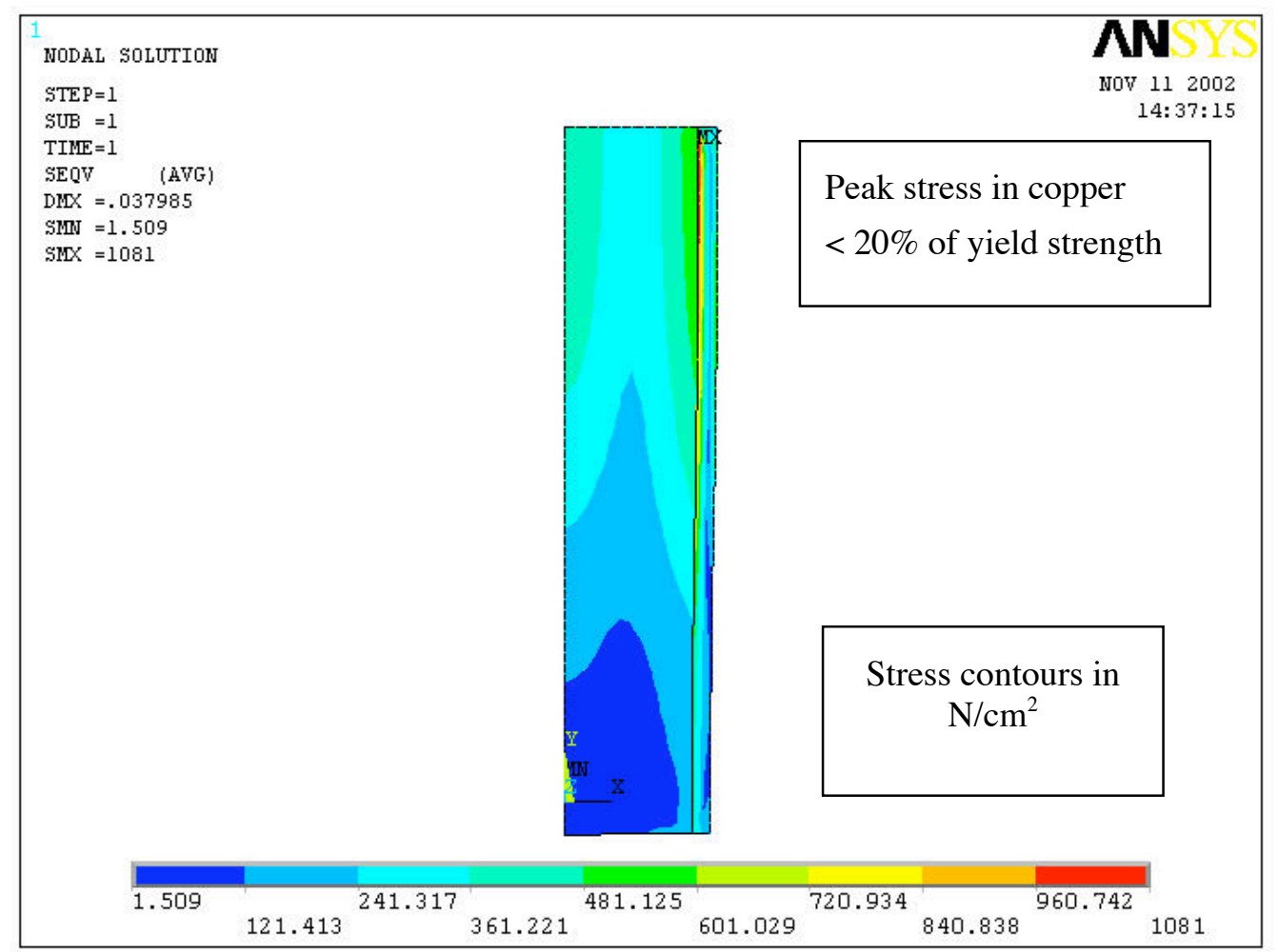

Figure 20-4 Stress contours (von Mises equivalent) for a carbon/copper beam dump, first $75 \mathrm{~cm}$.

The structural boundary conditions mirror the thermal case with a symmetry boundary applied at $x=75 \mathrm{~cm}$. The thermal stresses are very modest in comparison to the tensile strength of POCO graphite of $3500-7000 \mathrm{~N} / \mathrm{cm}^{2}(5000-10000 \mathrm{psi})$ and the yield strength of annealed OFHC copper of $7000 \mathrm{~N} / \mathrm{cm}^{2}(0.2 \%$ offset $)$.

On the basis of this initial study, the ability to construct a beam dump capable of withstanding the power of the proposed re-circulating linac running at an upgraded energy and elevated bunch charge $(3.1 \mathrm{GeV}, 3 \mathrm{nC})$ appears feasible. Peak temperatures and thermal stresses are relatively low for a reasonably sized water-cooled absorber constructed from carbon and copper. The design of the upstream windows and power dissipation at shower incipience may require that the beam be rastered. Radiation shielding, activation and cost issues are yet to be addressed.

\section{REFERENCES:}

[1] European Physical Journal C, Particle and Fields Vol. 15, pp.167-171, Nov 1, 2000. 


\section{CONVENTIONAL FACILITIES}

\section{Architectural Design}

The preferred site of the proposed femtosecond x-ray facility is in the Old Town area of Berkeley Lab in close proximity to the Advanced Light Source (ALS). The conventional facilities will consist of a two story building of approximately 125,000 gross square feet, and two separate free standing structures - a Cryogenic Plant, that will provide liquid helium for cooling of the accelerator, and an Utility Center, that will house heavy equipment for mechanical and electrical building systems support.

The high bay first floor of approximately 91,000 gross square feet will house the machine consisting of injector, linear accelerator, magnetic arcs, and production beam array of undulators and bend magnets, producing multiple beamlines. In addition to the machine, the first floor will provide experimental area, assembly areas, and laser rooms. There will be a continuous circulation pathway along the perimeter of the building to facilitate emergency exiting and dayto-day circulation between the functional areas. The structural bay housing the accelerator machine will be equipped with a 10-ton crane to facilitate installation and maintenance of the accelerator components. The experimental area will be served a by a monorail following the outline of experimental stations.

The second floor will house the control room, and a mixture of user labs consisting of wet laboratories, dry laboratories, and semi-clean rooms. Adjacent to laboratory areas will be offices, conference rooms, and a core of support facilities including cold rooms, dark rooms, equipment rooms, and similar research support spaces. Because of the extremely tight footprint, the mechanical and electrical equipment room will be also located on this level. Great care will be taken to isolate the electrical a mechanical equipment to minimize the vibration and acoustical impact. The structural design will provide for future expansion of the second floor.

The building design is taking advantage of the sloped topography of the site. The building will be accessible from Segre road and from an upper roadway circling the existing historically significant grove of redwood and pine trees. The main lobby, entered from the upper road, will be located on a mid-level with an elevator and stair connection to both first and second floors. A small lobby with a stair and an elevator will be provided on the opposite side of the building accessed from Segre Road and adjacent to truck access for staging of scientific equipment.

The exterior finish of low maintenance, factory preformed and painted, metal siding and clear glass with low emissivity coating will be compatible and complementary to the ALS complex. The roof will be a single sheet roofing membrane system with heat reflective coating to reduce solar gain. Metallic screens will conceal rooftop mounted mechanical exhaust equipment. An enclosed pedestrian bridge will link the building with ALS to facilitate crossdisciplinary collaboration of users in both facilities.

The femtosecond x-ray source project will make every effort to deliver an environmentally responsible design, construction, and operation. Its environmental impact will be reduced through sensitive site development; control of southern and western solar loads by means of blinds, sunshades, and recessed windows; water and energy conservation; and environmentally responsible building materials. 
The building will be protected by a sprinkler system connected to the LBNL fire alarm system. Smoke detection and other detection systems will be provided where required. The building will be designed in conformance with requirements for group ' $\mathrm{B}$ ' occupancy as defined by the California Building Code (CBC), Type II fire resistive construction, and seismic safety and fire safety code requirements. The building will comply with disabled accessibility requirements in accordance with the Americans with Disabilities Act (ADA).

The project is sited in conformance with the LBNL Site Development Plan. The project site is currently occupied by several old buildings used by the ALS, Accelerator and Fusion Research Division (AFRD), Engineering, EH\&S, Earth Sciences Division (ESD), and Facilities for research and other activities. These activities will be relocated.

\section{Structural Design}

Structural design shall account for all loads to which earth retaining systems and the building structure may be subjected including static, live, wind, seismic, etc. The design will comply with the requirements of the California Building Code (CBC) and LBNL's Lateral Force Design Criteria.

The first story of the building will be cast-in-place reinforced concrete slab-on-grade with tied back reinforced concrete retaining walls on the east, northeast and southeast sides. The west bay of the first story will contain cast-in-place concrete shielding structures at the beamline bends and be serviced by a top riding ten (10) ton crane. The upper floor and roof will be concrete slabs on metal deck supported by structural steel floor joists spanning between structural steel jack trusses supported by long span structural steel trusses and steel columns. In the transverse direction, the building will be a moment resisting frame and, in the longitudinal direction, various structural framing systems, such as eccentrically braced frames, concentrically braced frames and moment resisting frames, will be compared in the Title I design stage to determine the most suitable and cost effective system. The foundation system of the western portion of the building, including the shielding structures, will be drilled piers with reinforced concrete pier caps interconnected by tie beams. The foundation system of the eastern portion of the building will be isolated footings founded on bedrock and interconnected by tie beams.

Roofs shall be designed for a minimum mechanical equipment load of $50 \mathrm{lb} / \mathrm{sq} \mathrm{ft}$ or the actual weight of the mechanical equipment, whichever is greater. The elevated floor will be designed for a 125 psf live load.

Nonstructural building elements and the mechanical and electrical systems will be designed to accommodate calculated displacements due to lateral forces from wind or seismic forces.

\section{Mechanical Design}

\section{Heating, Ventilation and Air Conditioning System}

The experimental floor and the second floor laboratories and offices will be heated or cooled with tempered air to maintain inside design temperatures $76^{\circ} \mathrm{F} \pm 2^{\circ} \mathrm{F}$ in summer and $72^{\circ} \mathrm{F} \pm 2^{\circ} \mathrm{F}$ in winter. Precise temperature control at $25^{\circ} \mathrm{C} \pm 0.5^{\circ} \mathrm{C}\left(77^{\circ} \mathrm{F} \pm 0.9^{\circ} \mathrm{F}\right)$ will be maintained in 
shielding tunnels and laser rooms. Humidity control is not expected to be required. The minimum ventilation rate will be $1 \mathrm{cfm} / \mathrm{ft}^{2}$.

Air supply air handling units (AHU) will be housed in the second floor mechanical room. Exhaust fans will be mounted on the roof of the building within a screened area. The chillers and boilers will be located in the Utility Center. Chilled and heating hot water will be piped from the Utility Center to the AHU to provide pre-cooling and pre-heating. Final building heating will be provided by individual re-heat VAV boxes located throughout the building. Each unit will be provided with a dedicated temperature sensor to control re-heating and airflow from each individual VAV unit.

\section{Process Piping}

Mechanical utilities will include domestic cold and hot water (DC/HW), industrial cold and hot water (IC/HW), chilled water (CWS/R), low conductivity water (LCWS/R), compressed air (CA), vacuum (VA), fire sprinkler supply (FSS), sanitary sewer (SS), and helium (He) for cryogenic plant. The utilities on the main floor will be run in the trenches recessed in the concrete floor and terminate at valve stations at fixed locations compatible with accelerator assemblies or locations compatible with user needs. Liquid nitrogen will be provided by the cryogenic plant and will be piped or distributed in dewers to experimental stations and laboratories. Deionized water will be distributed through the second floor laboratories and polishing stations will be provided at points of use.

The following underground piping systems will require modifications and additions due to construction: low-conductivity water supply (LCWS), low-conductivity water return (LCWR), compressed air (CA), fire sprinkler supply (FSS), Sanitary sewer (SS), storm drain (SD), highpressure city water (HPCW), high-pressure natural gas (HPNG), treated water supply (TRWS), treated water return (TRWR). Temporary mechanical and underground utility services will be provided to all adjacent buildings and operations that will be affected by the construction work.

\section{Energy Conservation}

A dynamic computer analysis technique will be used to evaluate energy conservation alternatives and life cycle costs, determine design energy consumption, and determine passive and active solar effects. Use of renewable energy systems will be evaluated and utilized when shown to be cost effective. The heating and ventilating systems will be controlled by the Johnson Controls, Metasys digital control system. The control system will have central monitoring. Control strategies will include temperature control, VFD-controlled fans, chillers, cooling tower fans, pumps, night set back (with override), economizer, and other applicable energy conservation strategies.

\section{Electrical Design}

Power requirements for the building are projected at approximately $10 \mathrm{MGW}$, with 2-3 MGW for cryogenic plant, $3 \mathrm{MGW}$ for the accelerator and $\square 4 \mathrm{MGW}$ for user load. The building will be served by a dedicated electrical substation located on a separate pad in the vicinity of the Utility Center. The building will be served by 480/277 V and 208/120 V distribution systems with panelboards, branch circuit panels and step-down transformers located in dedicated electrical room. 
An emergency generator will be located in the Utility Center. Emergency power loads will include emergency egress lighting, fume hood and helium exhaust, and back up for compressors and other research equipment. UPS will provide power for sensitive computer and control equipment through emergency generator start-up time.

Lighting controls will be zoned, automated, and integrated with the Energy Management and Control System. Enclosed offices will be individually switched and controlled with occupancy sensors. Where natural light is available, two-level switching or photocell controls will be utilized. Task and specialty lighting will be provided where required.

Emergency lighting will be provided in laboratories, corridors, stairwells and other public areas by connecting selected fixtures of the general lighting system to standby power circuits. Building exit signs and emergency egress lighting units will be provided with 90 -minute backup battery packs and be connected to the standby power system.

The building will be equipped with telephone, communications, fiber-optic data circuits, paging and public address system, and building entry security system.

A complete building fire alarm system with extensions to the to the existing hill-wide LBNL fire alarm system will be provided.

\section{Gross Area Summary}

Building

First Floor

Second Floor

Separate Structures:

Cryogenic Plant

Utility Center

Switching Station

First Floor (Gross SF)

Experimental Area

Assembly Area

Laser Rooms (2@250 SF)

Machine

Lobby and Circulation

Second Floor (Net SF)

Laboratories, Research Support

Offices, Conference Rooms

Lounge, Lobby, etc.

Control Room

Mechanical/Electrical Room
$91,000 \mathrm{SF}$

$34,000 \mathrm{SF}$

$10,000 \mathrm{SF}$

$4,000 \mathrm{SF}$

$3,600 \mathrm{SF}$

$16,000 \mathrm{SF}$

$7,000 \mathrm{SF}$

$500 \mathrm{SF}$

$53,500 \mathrm{SF}$

$14,000 \mathrm{SF}$

$12,000 \mathrm{SF}$

$8,500 \mathrm{SF}$

$500 \mathrm{SF}$

$4,000 \mathrm{SF}$ 


\section{SITE SELECTION}

\section{Siting ASSESSMENT}

A two-step siting assessment process was undertaken in considering locations for the proposed facility. The first step of the siting process was initiated concurrent with discussions among the scientific personnel aimed at determining operational and research associations between the proposed building and other research activities at the Laboratory. In the initial or first pass assessment, sites that might physically accommodate this new building were identified. Possible sites were identified on the main Laboratory site, on lands immediately to the east of the Laboratory site, and in the neighboring cities of Richmond and Alameda (sites within a 15-mile radius of the Laboratory). Each of these sites was then evaluated as to their ability to accommodate the facility. All but four of the first-step sites were eliminated. Most were eliminated as essential utilities were not available in the general area or because the local jurisdiction had already made other plans for the site, and a number of the sites were eliminated as upon closer review they could not accommodate a building of the required size. The sites identified and considered in this phase are illustrated in Figure 22-1.

Four sites advanced for further consideration in the second phase; two on the Laboratory site and two at the University of California's Richmond Field Station. Each of the four sites were found to be feasible during this second phase review, however, in the end not all sites were ranked equally. One site, the Building 51 site, was not the top recommended site as a large former accelerator building remains on this site and this large structure is not scheduled to be demolished in a timeframe consistent with that preferred for the ultrafast dynamics facility. It was determined that acceleration of the demolition process would add approximately $\$ 40 \mathrm{M}$ to the cost of this project. The two sites in the City of Richmond are not the top recommended site as the research community determined that there are both scientific and operational benefits which would be lost in selecting a site approximately 15-30 minutes travel time from the Laboratory site. Moreover, it was determined that these sites would also require specially engineered foundations to ensure proper stability of the beam line floor. These two sites are adjacent to liquefaction zones and are on a sandy loam that carries vibration waves from adjacent railroad and industrial operations. 


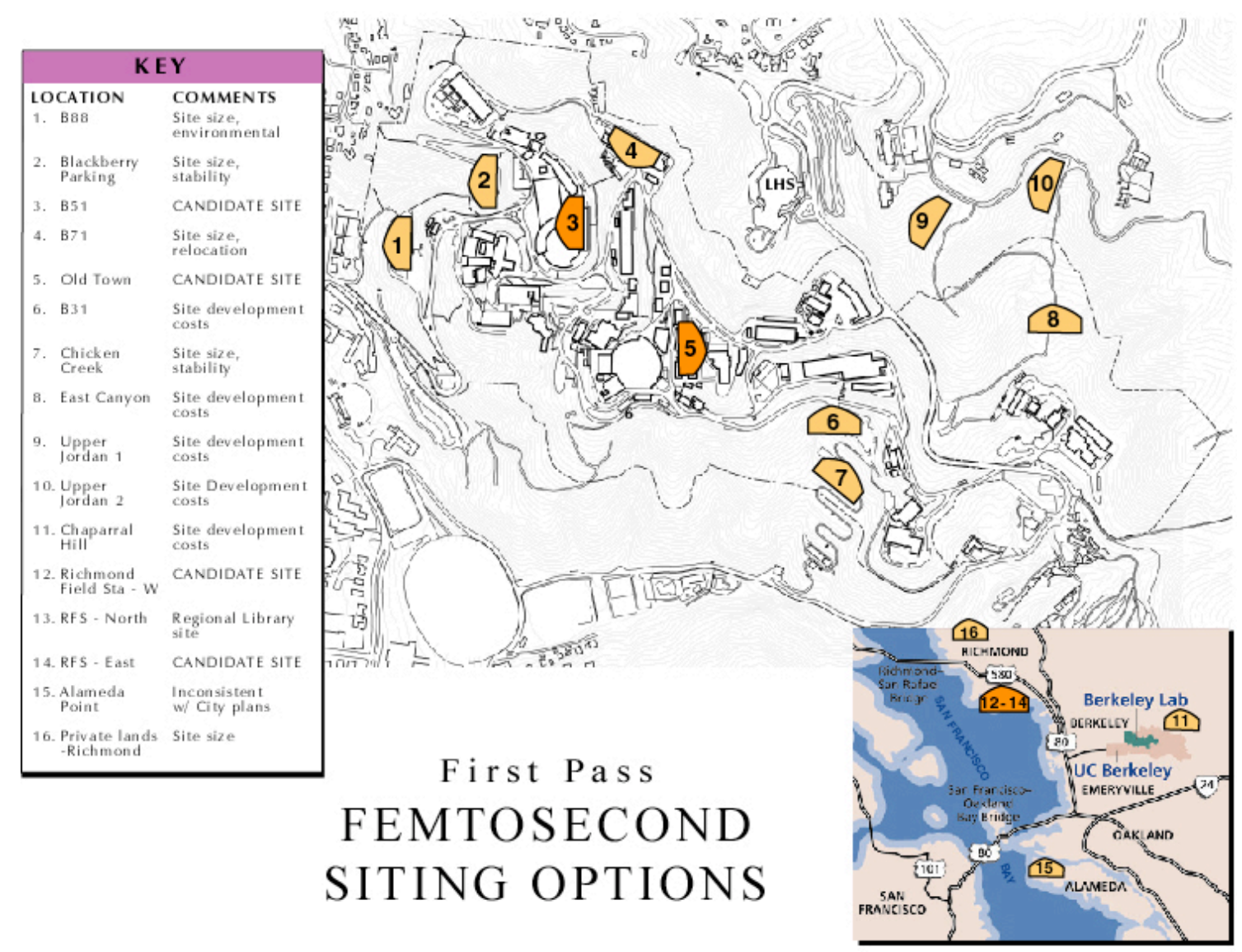

Figure 22-1 First pass sitting options

\section{Highest Ranked Site}

The highest ranked site for the proposed facility is immediately to the east of the Advanced Light Source (ALS). This site is in the Old Town area of Berkeley Lab (see Figure 22-2 below) and is currently occupied by a number of small buildings. The four primary factors driving this recommendation are summarized below:

Scientific and operational relationships with the ALS. Both the ALS and the proposed femtosecond dynamics facility are x-ray light sources dedicated to user experiments. The ALS and the femtosecond dynamics facility provide opportunities to conduct different but synergistically related research. An enclosed pedestrian bridge could link the femtosecond dynamics facility building with the ALS and facilitate both cross-disciplinary collaboration of users and efficient staffing of both facilities. Provision may be made for possible future beamline and end-station integration between the two facilities.

Utility Infrastructure. The proposed site is adjacent to a recently upgraded electrical substation with capacity to meet the needs of this facility as well as other future growth requirements of the Laboratory. The Laboratory obtains federal power via this substation. 
Stable Geology. The proposed site requires little excavation to reach stable bedrock. This site provides superior building stability, an important factor as beamline stability and alignment are paramount issues in the operation of this facility.

Site Renewal. The proposed Old Town site currently contains a number of small and difficult to effectively utilize W.W. II-era buildings. These buildings have provided over 50years of scientific service but no longer meet modern building codes or scientific research standards. Moreover, these buildings can not be cost-effectively upgraded to serve modern science and in general they are expensive to maintain in even a minimal operating condition. Thus siting at this location removes structures that are operational liabilities and replaces them with facilities fully capable of supporting the next generation of scientific research.

At this site, the building design can take advantage of a gently sloped topography. Excavation costs will be moderate. Moreover, there are design benefits as well. The experimental floor of the proposed building would be directly accessible from the adjacent road (Segre Road), while the upper floor offices and laboratories would be made directly accessible from another road (Road "R"). The main entrance to the upper level will be from a parking lot adjacent to a significant grove of redwood trees. The main lobby, entered from the upper road, will be located on a mid-level with an elevator and stair connection to both first and second floors.

The project is sited in conformance with Berkeley Lab's Long-Range Development Plan (LRDP); this LRDP foresaw and supports redevelopment of this site.

The Laboratory Site Remediation office conducted a review of sub-surface contamination records in the project area. This study concluded that scientific buildings such as those proposed are fully permissible at this location. It was also determined that standard precautions would be required for the identified contaminants. In general, the contamination is low-level and is generally a considerable distance below the proposed foundation elevation of the new building. A small-contaminated groundwater plume to the east (which is already being managed) can be controlled using standard technologies. Small and localized areas of soil contamination may exist under the foundations of some of the still standing buildings, we recommend a sampling program for these areas prior to excavation and removal of soil. The report also notes that an updated riskassessment may be required if the design of the building varies considerably from the assumptions that are at the basis of the current risk assessment. 


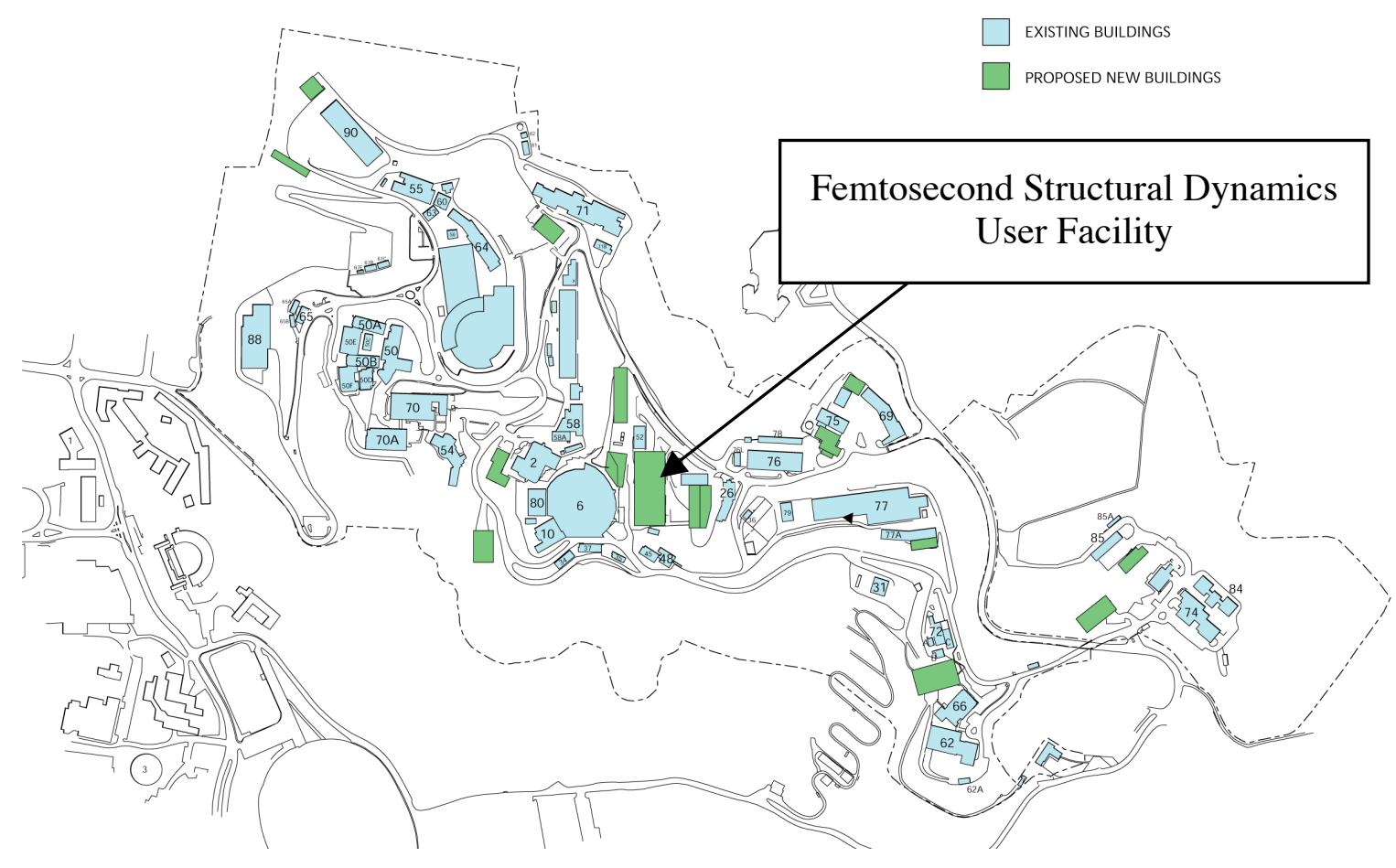

Figure 22-2 Top ranked location for a femtosecond dynamics facility at Berkeley Lab. The Advanced Light Source is the round building (6) immediately to the left of this femtosecond dynamics facility site.

\section{Groundwater Contamination}

The proposed accelerator site (Old Town or OT site) lies above a broad multi-lobed plume of groundwater contamination known as the Old Town Plume. Contaminants detected in groundwater are primarily halogenated non-aromatic volatile organic compounds(VOCs) (primarily industrial solvents [perchloroethene \{aka tetrachloroethene\} [PCE], trichloroethane [TCA], carbon tetrachloride], solvent degradation products [dichloroethene [DCE], dichloroethane [DCA], vinyl chloride, chloroform, etc], or both [trichloroethene [TCE]]). and lesser quantities of fuel hydrocarbons (diesel/kerosene and crude/waste oil).

Groundwater flow directions and maximum recorded groundwater elevations in the vicinity of the OT site have been determined; groundwater flow is northwestwards in the northern part, and southwestwards in the southern part of the site. The depth to groundwater varies considerably due to the differing hydraulic properties of the rocks beneath different parts of the site. The lobes of the Old Town Plume comprise several subplumes derived from multiple sources, which, primarily as a result of groundwater movement, have commingled to form the larger area of groundwater contamination. As a result, contaminated groundwater generally containing tens to hundreds of $\mu \mathrm{g} / \mathrm{L}$ (commonly referred to as parts-per-billion [ppb]) underlies most of the OT site. Following is a description of each lobe of the plume.

- The Building 7 lobe of the Old Town Plume contains the highest levels of contamination and has a principal contaminant source (the Building $7 \mathrm{sump}$ ) north of Building 7. This lobe lies 
entirely downgradient from the OT site (i.e. flow within the plume is away from the site), so is largely irrelevant to site development plans.

- The Building 52 lobe has substantially lower contaminant concentrations than the Building 7 lobe, but immediately underlies the northern part of the OT site. However, groundwater lies at a significantly greater depth than the expected foundation depth for the proposed accelerator, so no direct foundation to groundwater contact would occur.

- The Building 25A lobe also has relatively low contaminant concentrations compared to the Building 7 lobe. However, flow within this lobe is westward towards the center of the OT site, and recently a groundwater collection trench has been installed south of Building 44 to limit further westward migration of contaminated water. Analysis based on information from wells in the area shows that the groundwater highstand elevation (i.e. the highest recorded groundwater elevation) in this lobe intersects the OT site building footprint, indicating the potential for groundwater seepage into the excavation and contact between the building foundation and contaminated groundwater. However, it should be noted that the groundwater elevation as measured reflects the rise of groundwater into wells that have screens located below the elevation of the proposed building foundation, and, given the low permeability of rocks in this area, does not indicate that groundwater would in fact flow into the building foundation excavation. Insufficient data are currently available to evaluate whether or not groundwater seepage would occur into the excavation.

Development of the OT site would need to include mitigation of the following groundwater contamination impacts:

- Protection of construction workers from potential direct groundwater exposure while excavating the eastern portion of the foundation

- Capture, treatment and disposal of contaminated groundwater (if encountered) both during construction and after completion of the building

- Assessment and mitigation of toxic vapors that might migrate from groundwater through the soil into overlying buildings or into the excavation.

Generally, protection of construction workers would be assured through preparation of a construction risk management plan that included site monitoring and contingency plans. If groundwater was encountered during construction, capture and treatment would likely involve installation of extraction wells, sumps, or trenches, and construction of a temporary treatment system. It is likely that building subdrains (if needed) would need to be constructed as remediation systems to capture all groundwater that could potentially contact building foundations, and discharge it to a groundwater treatment system. The rainwater collection system for the building would need to be constructed so as to ensure that rainwater effluent was not mixed with groundwater in the subdrain system. A similar mitigation measure is currently employed at Building 46, where the previously existing subdrain has been adapted to capture the downgradient edge of the Building 52 lobe. 


\section{Human Health Assessment}

A human health risk assessment is currently in progress as part of Berkeley Lab's RCRA Corrective Measures Study. Preliminary results indicate that risks to building occupants at the OT site from vapor migration are generally below thresholds of concern (e.g. incremental risks less than $10^{-6}$ ) except for a very small area at the north end of the site. However, these results are based on the assumption of buildings located at the current ground surface and groundwater contamination remains at the present level. Since the Old Town site would apparently involve an unusually large excavation, the depths to groundwater beneath the building would decrease substantially from those utilized in the risk assessment, thus increasing risks. On the other hand, the recently completed Building 25 Corrective Measure may lower the level of contamination in groundwater over the next few years. Therefore, it would be necessary to reevaluate risks based on the proposed building design and the future groundwater contamination level, probably through a combination of modeling and vapor sampling. In the event that risks were shown to be significant, it is likely that regulatory agencies would require building construction modifications (e.g. vapor barriers beneath foundations, and possibly a continued monitoring plan) to mitigate potential risks.

\section{Soil Contamination}

Soil contamination associated with a number of historic sources exists within the proposed accelerator site. These include:

- $\quad$ PCBs and petroleum hydrocarbons at Area of Concern (AOC) 10-2, the former Building 52 hazardous materials storage area. Low levels of these contaminants are present in shallow soil (primarily <5 feet) in areas immediately adjacent to Building 52.

- $\quad$ PCBs, petroleum hydrocarbons and some metals at Solid Waste Management Unit (SWMU) 10-4, the Building 16 Former Waste Accumulation Area. Low levels of these contaminants were present in shallow soil on the slope west of Building 16.

Other soil contaminants are present at scattered locations at the OT site, but at levels less than regulatory cleanup standards for the site, and would not be triggers for construction or office worker protection measures. However, waste management regulations would effect disposal of such soils as wastes. Therefore, a soil management plan would need to be developed that including provisions for sampling, analysis, waste profiling, transportation and disposal of waste soil.

Given the long history of multiple uses of the OT site, it would be prudent to conduct sampling of currently inaccessible soil beneath buildings as they were demolished, with analyses conducted based on historical building use patterns. Proposed sampling scope and procedures could be included in the soil management plan discussed above. 


\section{TECHNICAL RISK ANALYSIS}

\section{Identification, ASSESSMent, AND Mitigation OF TeChnical Risks}

Key objectives of this feasibility study are:

1. Identify all of the technology challenges

2. Define the R\&D needed to ensure that all critical components have an acceptable risk by the start of the final/detailed design phase

The major systems and components have been reviewed and the associated technology, design and engineering, and manufacturing risks assessed. The criteria for identifying and assessing these technical risks are discussed below. The assessment of the technical risks is based on in-house expertise and information from key members of the accelerator technology community.

Our goal for the R\&D program is that all critical technologies reach the following Technology Maturity Levels (TML) by specific phases of the project development:

- TML Low to Medium by CD0, identification of mission need and beginning of conceptual design phase

- TML Medium to High by CD1, approved baseline and start of the detailed design phase

- TML High by start of the fabrication phase

Reaching these levels of technology maturity means reduced development risk, which translates into lower cost, schedule, and performance risks. Table 23-1 summarizes the results of the risk identification and qualitative assessment to-date and does not reflect the implementation of the risk reduction plans discussed later. Risk analysis will be ongoing throughout the project to monitor the status and progress of the risk reduction effort. 
Table 23-1 Summary of the technical risk assessment

\begin{tabular}{|c|c|c|c|c|}
\hline \multirow[t]{2}{*}{ Item } & \multicolumn{3}{|c|}{$\begin{array}{ll}\text { Risk } & \text { Factors }^{1} \\
\end{array}$} & \multirow[t]{2}{*}{ Comments $^{3}$} \\
\hline & $\overline{R \& D^{2}}$ & $\overline{D \& E^{2}}$ & Man. $^{2}$ & \\
\hline $\begin{array}{l}\text { RF photocathode } \\
\text { gun }\end{array}$ & $\mathrm{M}$ & $\mathrm{H}$ & $\mathrm{M}$ & $\begin{array}{l}\text { Warm copper RF photocathode gun demonstrated for low } \\
\text { operation rate. D\&E effort to operate reliably at high rate. }\end{array}$ \\
\hline Photocathode & $\mathrm{M}$ & $\mathrm{M}$ & $\mathrm{M}$ & $\begin{array}{l}\text { Recent results indicate good lifetime for } \mathrm{Cs}_{2} \mathrm{Te} \text { at high } \\
\text { vacuum. }\end{array}$ \\
\hline Photocathode laser & M & M & $\mathrm{L}$ & Laser pulse needs high temporal and amplitude stability. \\
\hline Flat beam & M & $\mathrm{L}$ & $\mathrm{L}$ & Uses demonstrated technique with standard optics. \\
\hline SC linacs & $\mathrm{M}$ & $\mathrm{M}$ & $\mathrm{M}$ & $\begin{array}{l}\text { Uses SoA SC RF cavities based on TESLA TTF; some } \\
\text { different parameters; much higher cryogenics loads. }\end{array}$ \\
\hline $\begin{array}{l}\text { Beam spreader } \\
\text { section }\end{array}$ & $\mathrm{L}$ & $\mathrm{M}$ & $\mathrm{L}$ & $\begin{array}{l}\text { Uses some specialized magnets based on experience. Tight } \\
\text { geometry poses } \mathrm{D} \& \mathrm{E} \text { challenge. }\end{array}$ \\
\hline HGHG FELS & M & M & M & $\begin{array}{l}\text { Successful proof-of-principle experiment in IR region } \\
\text { promising for the generation of higher energy photons. }\end{array}$ \\
\hline Deflecting cavities & $\mathrm{H}$ & $\mathrm{H} / \mathrm{M}$ & $\bar{M}$ & $\begin{array}{l}\text { Uses SoA SC RF cavities. Stringent requirements for } \\
\text { stability, HOM and LOM damping. }\end{array}$ \\
\hline Lattice magnets & $\mathrm{L}$ & $\mathrm{L}$ & $\mathrm{L}$ & Uses standard water-cooled electromagnets. \\
\hline Undulators & $\mathrm{M}$ & $\mathrm{H} / \mathrm{M}$ & $\mathrm{H} / \mathrm{M}$ & $\begin{array}{l}\text { Trade between permanent and SC undulators is still open: } \\
\text { performance vs. risks. May use several different undulators. }\end{array}$ \\
\hline Beamline optics & $\mathrm{M}$ & M & $\mathrm{L}$ & $\begin{array}{l}\text { SoA optics with some different parameters for pulse } \\
\text { compression. }\end{array}$ \\
\hline Beam dump & $\mathrm{L}$ & $\mathrm{L}$ & $\mathrm{L}$ & Similar to existing beam dumps. \\
\hline $\begin{array}{l}\text { Diagnostics and } \\
\text { instrumentation }\end{array}$ & $\mathrm{L}$ & $\mathrm{M}$ & $\mathrm{L}$ & $\begin{array}{l}\text { Use of demonstrated techniques with some new } \\
\text { requirements for measurement of beam tilt. }\end{array}$ \\
\hline Synchronization & $\mathrm{H}$ & $\mathrm{H}$ & $\mathrm{M}$ & $\begin{array}{l}\text { Tight requirements for } \mathrm{x} \text {-ray pulses, electron bunches, and } \\
\text { pump lasers. }\end{array}$ \\
\hline Pump lasers & $\mathrm{M}$ & $\mathrm{M}$ & $\mathrm{L}$ & Tight synchronization required with deflecting cavities. \\
\hline Control systems & $\mathrm{L}$ & M & $\mathrm{L}$ & $\begin{array}{l}\text { Faster acquisition rates than ALS. Important to efficient } \\
\text { commissioning and operations. }\end{array}$ \\
\hline $\begin{array}{l}\text { Low-level RF } \\
\text { systems }\end{array}$ & $\mathrm{M}$ & $\mathrm{M}$ & $\mathrm{L}$ & $\begin{array}{l}\text { Adjustable tuners save power. Some development required } \\
\text { using standard techniques. }\end{array}$ \\
\hline Vacuum systems & $\mathrm{L}$ & $\mathrm{L}$ & $\mathrm{L}$ & Similar to existing designs. \\
\hline
\end{tabular}

Notes:

1. The risk assessment reflects the present status of major components

2. R\&D: Research and Development, D\&E: Design \& Engineering, Man: Manufacturing

The criteria for the assigned risk factors are:

$\mathrm{H}$ (HIGH): Concepts and/or prototypes need to be developed.

M (MEDIUM): Similar components or subsystems have been successfully demonstrated or require some extrapolations from existing designs.

L (LOW): Subsystems and components are essentially identical to existing and proven designs.

3. Details are presented in the text.

Specific risk mitigation plans are being developed and implemented for all the highand medium-risk items. Several key components need extensive R\&D. A number of research institutions are involved in parallel technology development programs, and participation in several of these efforts has begun. Where necessary, additional and 
distinct $R \& D$ efforts are proposed to meet specific requirements. Our design trade studies and analyses support these technology development activities in allocating resources in the most effective manner, to increase the probability of a successful project that meets the user needs with realistic cost and schedule.

If the basic technology is available but there is risk stemming from the advanced application of the technology multiple design approaches will be carried until a decision point is reached. If the primary risk is in the manufacturing area, multiple vendors will be carried until one proves the capability to produce the element on time in the quantity required. Rigorous acceptance testing of individual assemblies (lasers, RF power supplies, magnets, instrumentation, etc) will help to minimize these risks.

Integration is a significant risk for our machine because successful operation requires the critical coupling of different elements (lasers, RF subsystems, optics, etc). Interface and integration issues will be identified and fully integrated before the hardware/software is shipped for integration with other subsystems. Adequate commissioning time is also essential.

\section{RF photocathode gun}

Experiments at the A0 photoinjector at the Fermilab have already demonstrated that RF photocathode guns can produce electron beams with high intensity and low emittance for a repetition rate in the range of 10 to $100 \mathrm{~Hz}$. The required gun must operate reliably at a significantly higher repetition rate of $\sim 10 \mathrm{kHz}$. Control of space charge effects, thermal emittance, quantum efficiency, cavity thermal design, and overall systems reliability are significant challenges. Early results are encouraging; but, additional R\&D is required to develop a design that meets the full requirements.

The gun design has a significant impact on the achievable beam current and emittance, and because of its critical importance in defining the photon beam flux and pulse duration, the engineering and design of the photocathode gun is classified as a highrisk item.

To mitigate risk factors the following actions are being pursued:

1. Participation in the experimental program and development of the FNPL photoinjector at Fermilab.

2. Active involvement in other photocathode RF gun experiments.

3. In-house design of a high-gradient, high-duty RF photocathode gun.

Our assessment is that, with additional R\&D, there is a high probability of successful design and construction of a photoinjector with the parameters specified. Preliminary results are very encouraging and indicate that the requirements can be achieved at a repetition rate of $10 \mathrm{kHz}$ using a reliable design with adequate safety margins. If the baseline concept does not achieve the required emittance, beam collimation may provide sufficient beam quality. 


\section{Deflecting cavities}

R\&D on superconducting deflecting cavities is on-going at Fermilab, KEK, and Cornell University. The Fermilab group has designed, fabricated and tested a single cell cavity, a five-cell cavity, and a thirteen-cell cavity. These cavities are designed to achieve a $5 \mathrm{MV} / \mathrm{m}$ deflecting electric field at $3.9 \mathrm{GHz}$, at a low rep rate of $5 \mathrm{~Hz}$, and low duty cycle of $0.7 \%$. The single cell cavity test has already achieved $9.5 \mathrm{MV} / \mathrm{m}$ deflecting gradient with $\mathrm{Q}_{\mathrm{o}}$ of $1 \times 10^{9}$, while the five-cell cavity has achieved $1.5 \mathrm{MV} / \mathrm{m}$ deflecting gradient with $\mathrm{Q}_{0}$ of $2 \times 10^{9}$. Additional $\mathrm{R} \& \mathrm{D}$ and design and engineering is required to achieve the specified 8.5 MV deflecting voltage for $\mathrm{CW}$ operations. Additionally, there is a need to investigate and resolve several technical issues including parasitic mode damping, multipacting suppression, and input power coupling, and thus these components are considered a high risk item.

A preliminary cavity design for a 7-cell cavity has been completed using the computer simulation codes MAFIA and URMEL. Further investigations including a 3D MAFIA model of the RF coupler and analysis of its effects on the parasitic modes are in progress. Collaborations with Fermilab, TJNAF, DESY, and ACCEL GmbH have been initiated. Based on the preliminary data, this effort is judged to have a high probability of success, further details are presented in section 8-Superconducting RF.

\section{Undulators}

Since the undulator trade studies are still to be completed, our discussion of technical risks is generic rather than specific to a baseline design. Technical risks are driven by the challenging requirements for tight machining tolerances, tunable magnetic field strength ranging, long length, tight beam control and demanding instrumentation. Conventional permanent magnet undulators may provide a maximum magnetic field of $\sim 1.0 \mathrm{~T}$. To achieve higher undulator magnetic fields, a superconducting short period undulator is being considered. ACCEL Instruments $\mathrm{GmbH}$ and the Karlsruhe Research Center have developed a first test device of a superconducting in-vacuum undulator and are now developing a prototype to resolve a number of identified issues including manufacturing and instrumentation. The results are encouraging; but significant R\&D is required to develop a practical a full-length, short period superconducting undulator. While less of a technical challenge, a permanent magnet short period long undulator still faces the design and manufacturing issues: tight mechanical tolerances, thermal environmental control, beam position monitoring, alignment stability, and radiation dose management.

\section{Synchronization}

The accurate timing of the femtosecond $x$-ray probe pulse with respect to the experimental pump laser that excites the processes in the sample under study is critical for the investigation of structural dynamics in the femtosecond regime. This requires that the compressed x-ray pulse and the external laser be synchronized to approximately $50 \mathrm{fs}$. Our overall strategy is to lock all laser signals to a common laser master oscillator and 
derive all RF signals it. Jitter reduction arising from the hard x-ray pulse compression scheme gives a significant advantage. Details are presented in Chapter 19Synchronization.

The design and engineering challenges include:

- Stabilizing several laser oscillators to the laser master oscillator

- Stable distribution of RF signals derived from the master oscillator, throughout the machine

- Mechanical stability of RF pickups, cables, and connectors

- Drift and timing jitter of the RF photocathode laser

- Phase and amplitude jitter in the pulsed RF gun systems

Synchronization is considered to be high risk because of the stringent requirements and the design and engineering challenges.

\section{Superconducting linacs}

An active $R \& D$ effort is proceeding that includes extensive simulations and analytical studies of (i) collective effects including HOM damping and multipacting suppression, (ii) input power coupler design, and (iii) cryogenic systems. Collaborations with Fermilab, TJNAF, DESY, and ACCEL GmbH have been initiated. Although the TESLA superconducting cavity appears well suited to our requirements, we carry the CEBAF upgrade module as a viable alternative. Indications are that requirements will be achieved with the proposed plan. 